Portland State University

PDXScholar

Spring 9-29-2017

\title{
Controlling Water Exchange Kinetics and Improving ParaCEST Imaging
}

Jacqueline R. Slack

Portland State University

Follow this and additional works at: https://pdxscholar.library.pdx.edu/open_access_etds

Part of the Chemistry Commons, and the Medicine and Health Sciences Commons Let us know how access to this document benefits you.

Recommended Citation

Slack, Jacqueline R., "Controlling Water Exchange Kinetics and Improving ParaCEST Imaging" (2017). Dissertations and Theses. Paper 3945.

https://doi.org/10.15760/etd.5829

This Thesis is brought to you for free and open access. It has been accepted for inclusion in Dissertations and Theses by an authorized administrator of PDXScholar. Please contact us if we can make this document more accessible: pdxscholar@pdx.edu. 
Controlling Water Exchange Kinetics and Improving ParaCEST Imaging

by

Jacqueline R Slack

A thesis submitted in partial fulfillment of the requirements for the degree of

\author{
Master of Science \\ in \\ Chemistry
}

Thesis Committee:

Mark Woods, Chair

Robert Strongin

David Stuart

\title{
Portland State University \\ 2017
}


(C) 2017 Jacqueline R Slack 


\begin{abstract}
Generating MR image contrast from exogenous contrast media though chemical exchange saturation transfer (CEST) offers several exciting new possibilities, such as multicolored imaging, the interleaving of pre- and post-contrast images, and the potential to perform ratiometric metabolic imaging. The major limitation of the deployment of CEST imaging is the comparatively high detection limits of exogenous agents and particularly at the low $\mathrm{B}_{1}$ power levels required to meet SAR requirements. The large chemical shifts afforded by paramagnetic (paraCEST) agents permit more rapid exchange kinetics and therefore potentially more effective contrast agents. Despite comparatively large chemical shifts, many $\mathrm{Ln}^{3+}$ DOTA-tetraamide (DOTAM) chelates traditionally investigated as CEST agents are predicted to have exchange kinetics that are considerably faster than optimal at very low $\mathrm{B}_{1}$ powers. This work explores two methodologies for slowing water exchange kinetics in $\mathrm{Ln}^{3+}$ DOTAM chelates and improving CEST imaging: structural manipulation and encapsulation. In the first method, rigid $\mathrm{Ln}^{3+} \mathrm{NB}-\mathrm{DOTAM}$ chelates with hydrophobic amide substituents was thoroughly studied using NMR spectroscopy techniques in order to assess their ability to produce CEST contrast at low $\mathrm{B}_{1}$ power levels. NMR techniques utilized included ${ }^{1} \mathrm{H}$ NMR, variable temperature, COSY, and CEST experiments. The phenyl amide substituent in the pseudo-axial position afforded chelates with considerably slow water proton exchange rates and appreciably more CEST contrast than isomeric chelates with the amide substituent in the pseudo-equatorial position. The second method involved characterizing a vesicle system to be used for encapsulating a $\mathrm{Ln}^{3+}$ DOTAM chelate. The vesicles prepared were analyzed using the following NMR
\end{abstract}


techniques: ${ }^{1} \mathrm{H}$ NMR, $\mathrm{T}_{1}$, shift reagent, and CEST experiments. The vesicle system chosen for study did not afford slow water exchange kinetics to enhance CEST contrast. A second vesicle system was attempted but the vesicle synthesis was difficult, parameters studied were not optimized, and the second system did not exhibit slow water exchange with the limited amount of experiments run and data collected. 


\section{DEDICATION}

In memory of Carol Shirey, whose fight led me down this path.

In memory of Jack Nicholson, James Slack, and Dean Slack, whose fights pushed me forward and kept me motivated.

For my family, my friends, and every person fighting for their life. 


\section{ACKNOWLEDGMENTS}

First, I would like to thank my thesis advisor, Dr. Mark Woods, for creating a family in his research group, for teaching me about plumb bobs, and for encouraging me to strive to be the best that he believed I could be.

Besides my advisor, I would like to thank my former PhD committee (Dr. Theresa McCormick, Dr. Todd Rosenstiel, Dr. Charles Springer) and my current MS committee (Dr. Robert Strongin, Dr. David Stuart) for the support they have given me and for the time and effort they have invested in my education.

Dr. Andrea DeBarber, Dr. Robert Jensen, and Dr. David Peyton deserve many thanks for their assistance in acquiring and interpreting spectral data. Dr. Andrea Goforth, Dr. David Stuart, Dr. Dirk Iwata-Reuyl (and graduate students James Barnes, Thomas Seidl, Ben Turner, Hayden Winter) deserve many thanks for allowing me to use their laboratory facilities and instrumentation.

My friends and colleagues in the Chemistry Department here at Portland State have been invaluable, and it is impossible for me to list everyone out. From thoughtful gifts, occasional surprise treats, genuine hugs and smiles, handwritten notes of encouragement, endless inside jokes, and many shared meals, to heartfelt conversations, mutual complaining sessions, and actual breakdowns, you are true friends. Thank you. I would have lost my mind without all of you. 
I want to thank my former and current Woods group members (Dr. Benjamin Webber, Dr. Julian Haigh, Dr. Annah Farashishiko, Dr. Katherine Payne, Guadalupe Camacho, Joseph Armstrong, Lauren Rust, Karley Maier, Christina Miller) for their never ending support and encouragement, their tough-love and advice, the required daily dosage of laughter, and all of the adventures over the last 6 years. Without you, I would not be writing this document.

Carin: Thank you for dissolving my fears and anxieties, for letting me talk it out with you when I was stuck (even if you didn't understand what I was talking about), and thank you for pushing me when I couldn't push anymore.

Pat: Thank you for the countless texts, hugs, cheesy jokes, and for all of the food. Thank you for being patient and making sure I got to the finish line. Thank you for taking care of me, for loving me, and for being in my world.

I owe the biggest debt of gratitude to my family who continues to support me unconditionally, encourage me, and love me on the darkest of days, and who have graciously put up with me being away for so long. 


\section{TABLE OF CONTENTS}

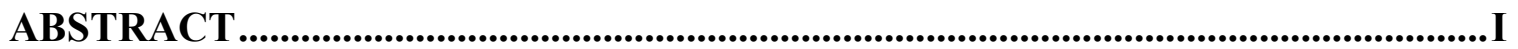

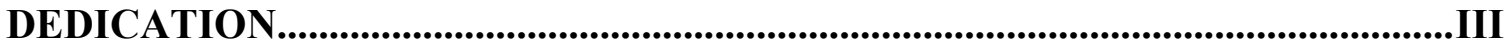

ACKNOWLEDGMENTS .............................................................................................. IV

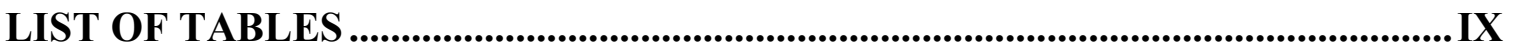

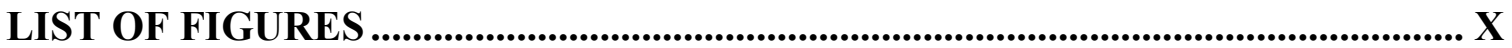

CHAPTER 1. INTRODUCTION OF MEDICAL IMAGING AND MAGNETIC

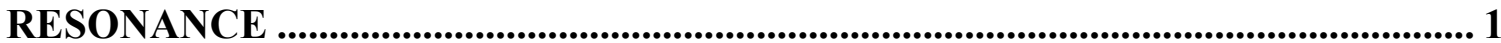

1.1. The 123s of Medical Imaging ................................................................................ 1

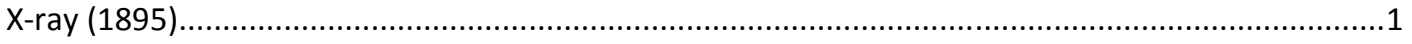

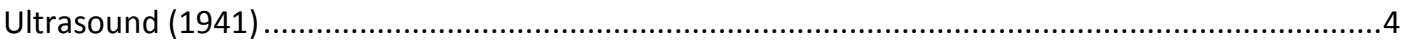

Positron Emission Tomography (PET) / Single-Photon Emission Computed Tomography (SPECT)

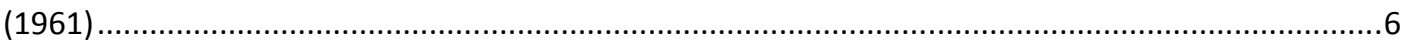

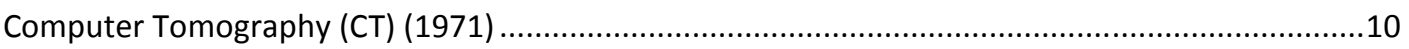

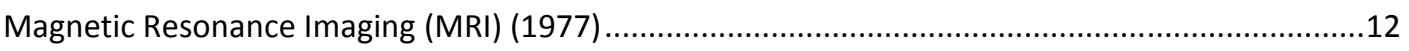

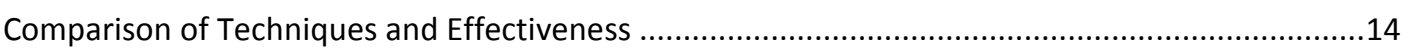

\subsection{Nuclear Magnetic Resonance (NMR) and Magnetic Resonance Imaging}

\section{(MRI) 15}

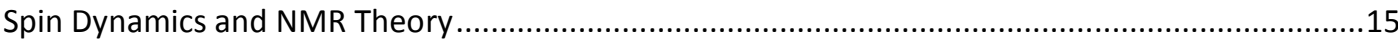

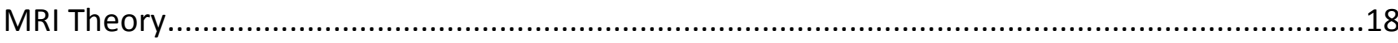

1.3. Contrast Agent Structure and Function ....................................................... 28

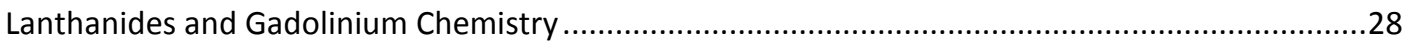

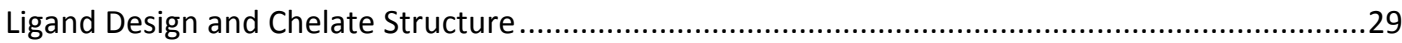

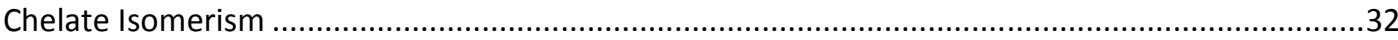

1.4. Progressing the MRI Technique......................................................................... 39

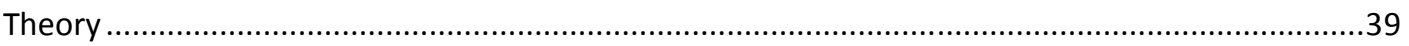

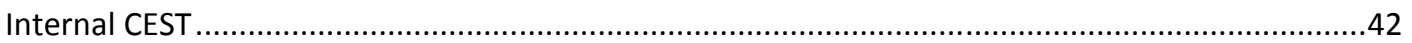

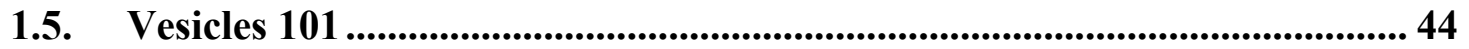

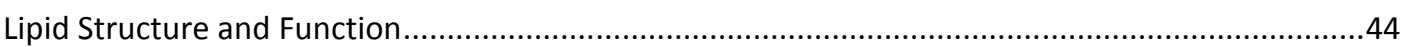

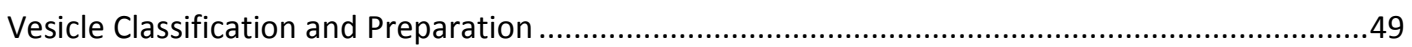

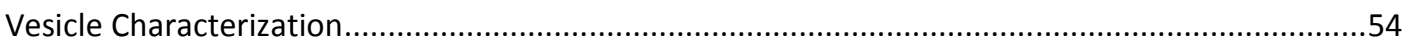




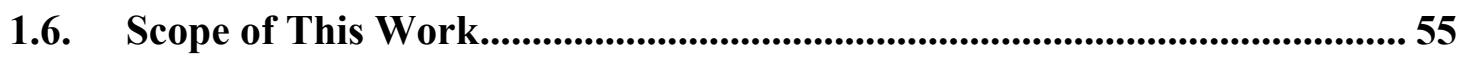

CHAPTER 2. STRUCTURAL MANIPULATION PROJECT ..................................57

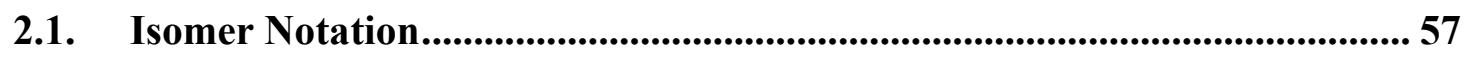

2.2. Motivation - Carboxylic Derivatives1 ............................................................. 62

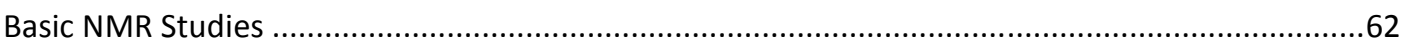

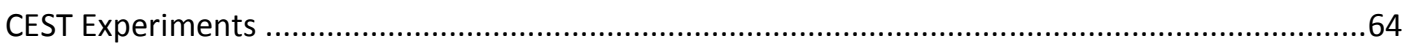

2.3. Studied Chelates - Phenyl Derivatives ${ }^{2}$ ….......................................................... 68

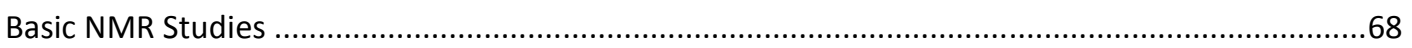

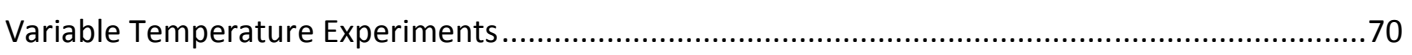

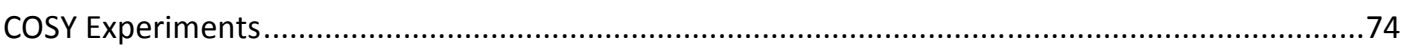

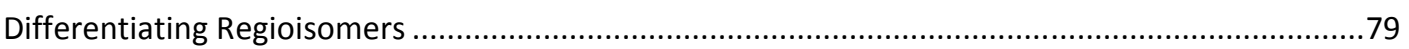

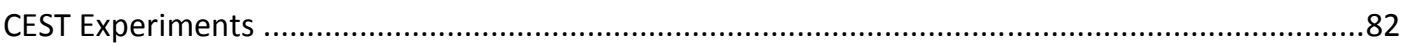

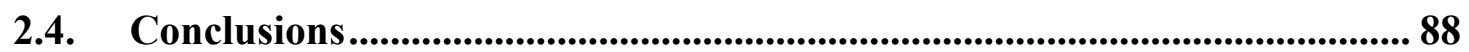

CHAPTER 3. ENCAPSULATION USING VESICLES............................................... 89

3.1. Vesicle Characterization ..................................................................................... 89

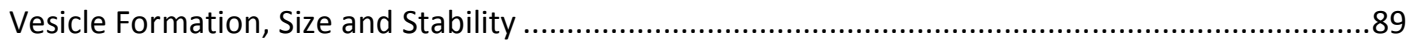

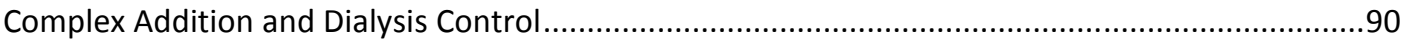

3.2. T1 Analysis of Vesicle Systems ............................................................................ 95

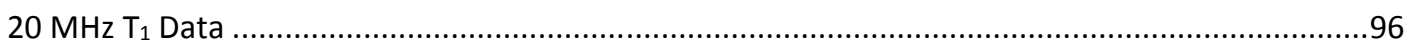

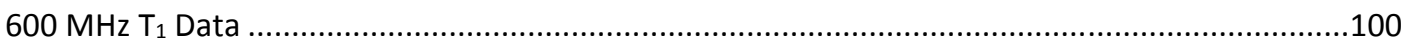

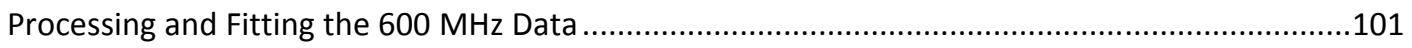

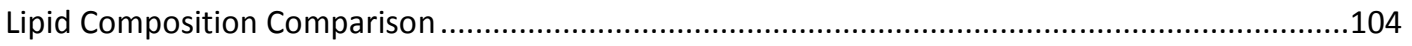

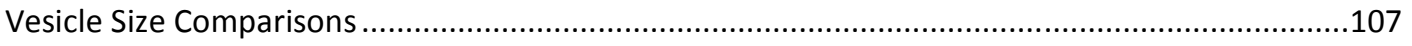

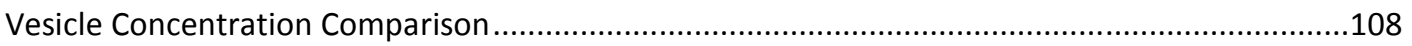

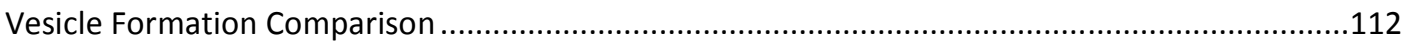

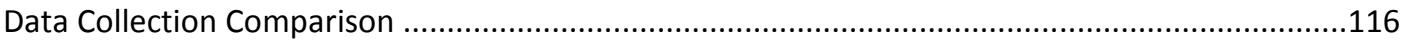

Finding the Encapsulated Volume of $100 \mathrm{~nm}$ Vesicles .....................................................................116

3.3. CEST Analysis of Vesicle Systems ................................................................... 118

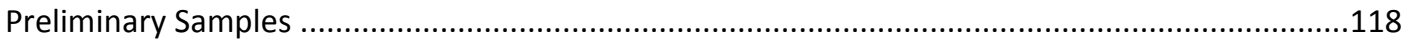

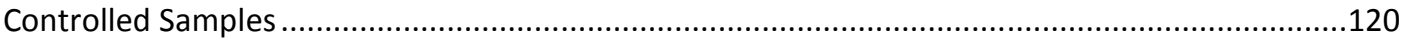

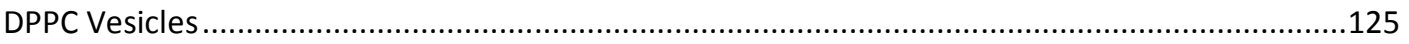

3.4. Conclusions .............................................................................................................. 126 
CHAPTER 4. MATERIALS AND METHODS...................................................... 128

4.1. Ln NB-DOTAM Project ............................................................................... 128

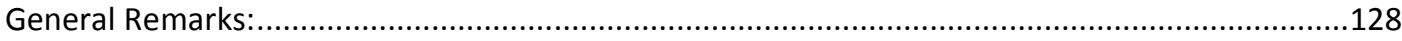

$(1 R, 4 R, 7 R, 10 R)-\delta, \delta^{\prime}, \delta^{\prime \prime}, \delta^{\prime \prime \prime}$-Tetramethyl-[2-(S)-(p-nitrobenzyl)-1,4,7,10-tetraazacyclo dodecane

]-1,4,7,10-tetra (2-phenylethyl)acetamide dihydrochloride salt (S-RRRR-2) .................................129

$(1 S, 4 S, 7 S, 10 S)-\delta, \delta^{\prime}, \delta^{\prime \prime}, \delta^{\prime \prime \prime}-$ Tetramethyl-[2-(S)-(p-nitrobenzyl)-1,4,7,10-tetraazacyclo dodecane ]-

1,4,7,10-tetra (2-phenylethyl)acetamide dihydrochloride salt (S-SSSS-2) ....................................130

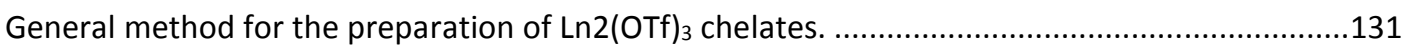

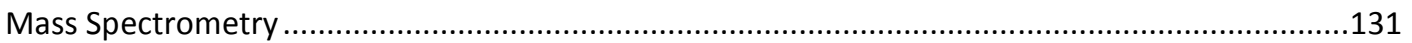

4.2. Vesicle Project ........................................................................................ 135

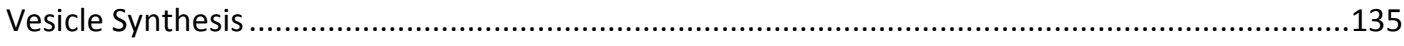

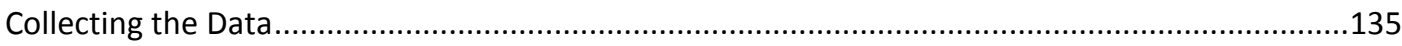

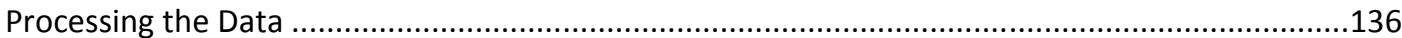

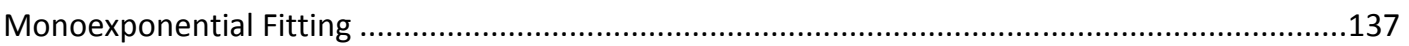

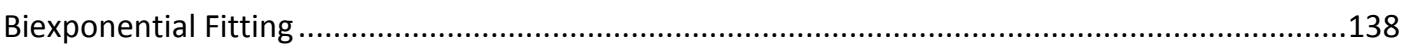

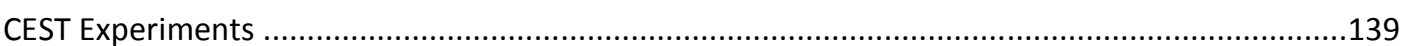

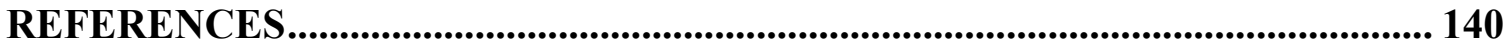




\section{LIST OF TABLES}

TABLE 1.5.1. PHOSPHOLIPID TRANSITION TEMPERATURE. ............................. 46

TABLE 2.3.1. EU3/4 SAP ISOMER \%CEST VALUES AND T $T_{M}$ VALUES............... 85

TABLE 3.2.1. CHANGING PARAMETERS FOR COMPARISONS AND BETTER

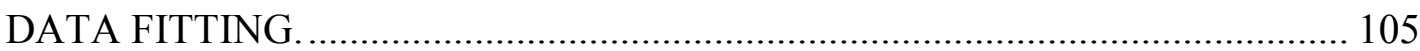




\section{LIST OF FIGURES}

FIGURE 1.1.1. THE FIRST HUMAN X-RAY PERFORMED. ..................................... 1

FIGURE 1.1.2. THE FIRST ANATOMIC ULTRASOUND PERFORMED.................... 4

FIGURE 1.1.3. THE FIRST CEREBRAL PET SCAN, PERFORMED IN 1961 WITH

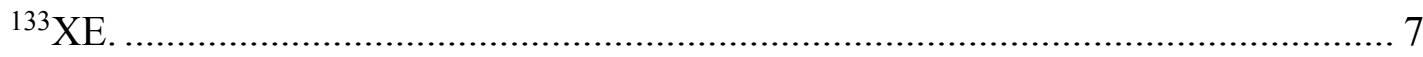

FIGURE 1.1.4. THE FIRST CT SCAN IMAGE OF A BRAIN TUMOR....................... 11

FIGURE 1.1.5. THE FIRST MRI IMAGE OF RAYMOND DAMADIAN'S TORSO, AROUND T7/T8, PERFORMED IN 1977........................................................ 13

FIGURE 1.2.1. SPINS IN A WEAK MAGNETIC FIELD AND A STRONG

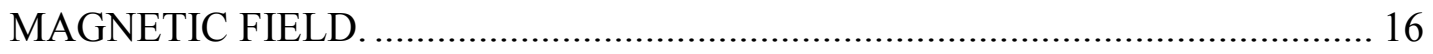

FIGURE 1.2.2. THE BOLTZMANN DISTRIBUTION, DEPICTING THE ENERGY DIFFERENCE BETWEEN THE A AND B ENERGY STATES AND THE INCREASING DIFFERENCE BETWEEN THEM WITH AN INCREASING

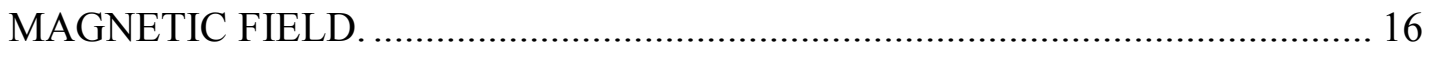

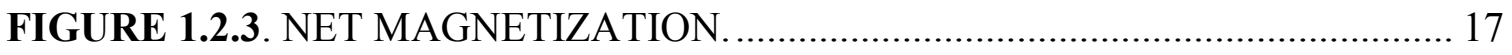

FIGURE 1.2.4. NET MAGNETIZATION IN THE X-Y PLANE AFTER AN RF PULSE, $\mathrm{B}_{1}$ 17

FIGURE 1.2.5. $T_{1}$ AND $T_{2}$ RELAXATION ........................................................... 18

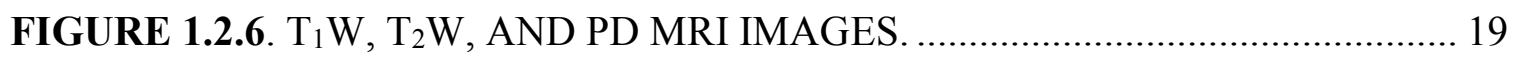

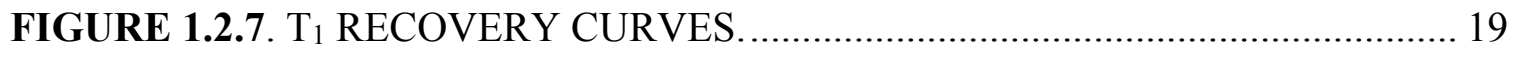

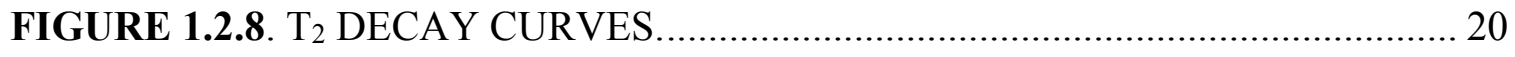

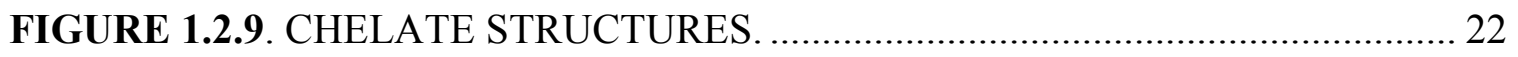


FIGURE 1.2.10. SATURATION MECHANISM................................................. 23

FIGURE 1.2.11. CEST MECHANISM............................................................... 23

FIGURE 1.2.12. SOURCES OF CEST IMAGING. ................................................ 25

FIGURE 1.2.13. WATER EXCHANGE RATE VS. MAXIMUM CEST EFFECT ACHIEVED AT VARIOUS SATURATION PULSE POWER LEVELS. ............. 26

FIGURE 1.2.14. CEST AGENT STRUCTURE................................................... 27

FIGURE 1.3.1. THE LANTHANIDE CONTRACTION ........................................... 28

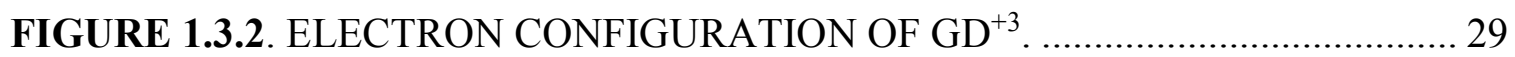

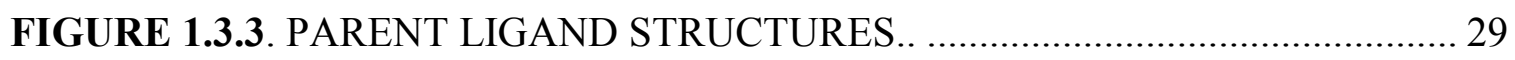

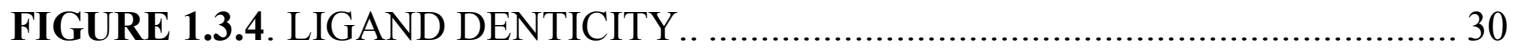

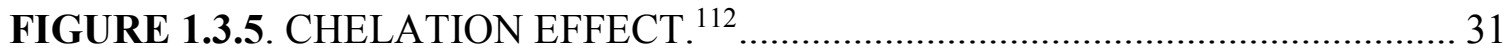

FIGURE 1.3.6. VISUALIZING THE CHELATES............................................ 32

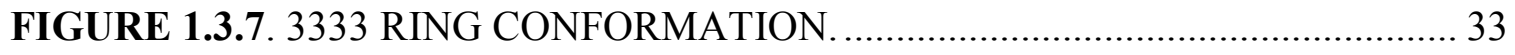

FIGURE 1.3.8. SAP AND TSAP ISOMER INTERCONVERTION. .......................... 34

FIGURE 1.3.9. UNSUBSTITUTED ISOMER FLOWCHART................................ 35

FIGURE 1.3.10. RING SUBSTITUTION ISOMER FLOW CHART.......................... 36

FIGURE 1.3.11. A POSITION ARM SUBSTITUTION ISOMER FLOWCHART ..... 36

FIGURE 1.3.12. REGIOISOMERS FLOW CHART.. ......................................... 37

FIGURE 1.3.13. DELTA SUBSTITUTION EFFECTS............................................ 38

FIGURE 1.4.1. SPECIALIZED MRI EXAMS. ................................................ 40

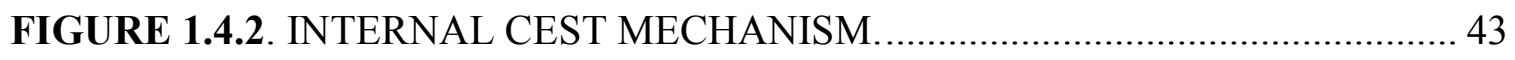

FIGURE 1.5.1. LIPID STRUCTURE AND PHOSPHOLIPID BILAYER .................. 44 
FIGURE 1.5.2. NATURALLY OCCURRING PHOSPHOLIPID HEAD GROUPS.... 47

FIGURE 1.5.3. SATURATED LIPIDS. EXAMPLES OF SATURATED LIPIDS. ...... 48

FIGURE 1.5.4. UNSATURATED LIPIDS ...................................................... 48

FIGURE 1.5.5. CHOLESTEROL STRUCTURE. ................................................. 49

FIGURE 1.5.6. VESICLE CLASSIFICATION. ................................................ 50

FIGURE 1.5.7. VESICLE FORMATION MECHANISM. ....................................... 51

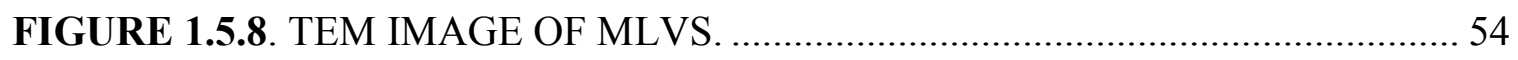

FIGURE 1.5.9. INTRALIPOSOMAL NMR ANALYSIS ....................................... 55

FIGURE 2.1.1. REFERENCE CHELATE FOR NAMING NOTATION, “CORNER”-

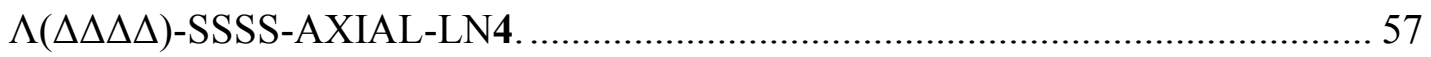

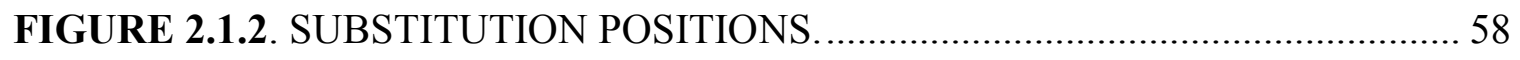

FIGURE 2.1.3. PSEUDOAXIAL AND PSEUDOEQUATORIAL POSITIONS IN

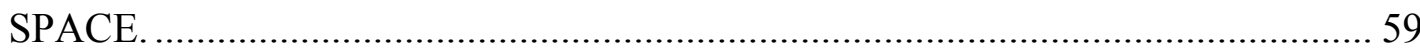

FIGURE 2.1.4. ISOMER NAMING FLOWCHART ............................................. 59

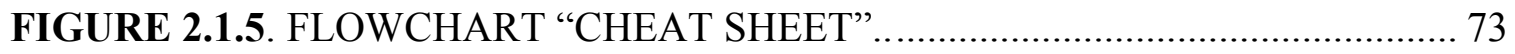

FIGURE 2.2.1. CARBOXYLATE LN $^{3+}$ DOTAM CHELATES STUDIED.................... 62

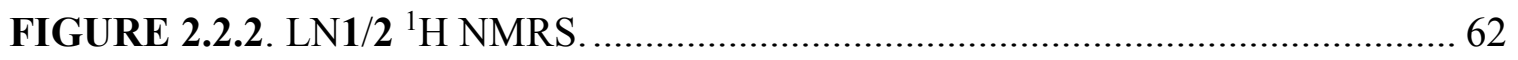

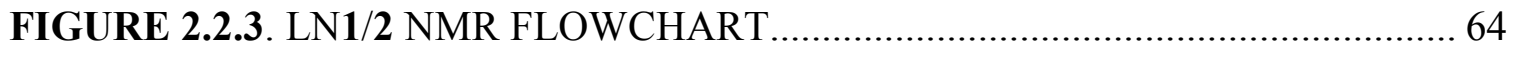

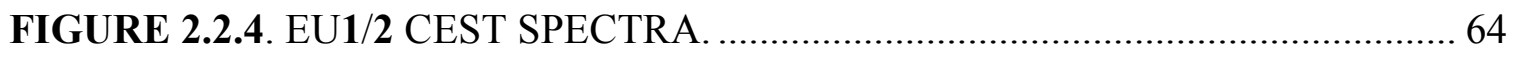

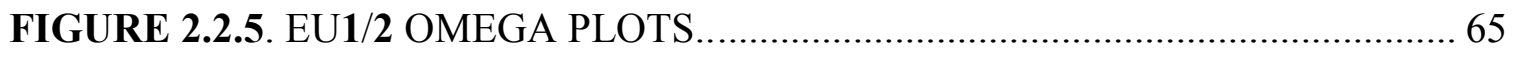

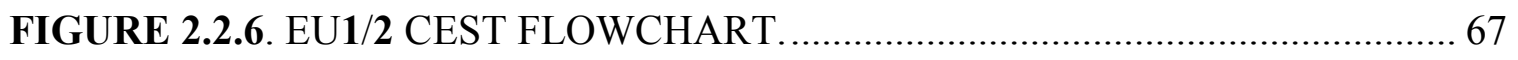

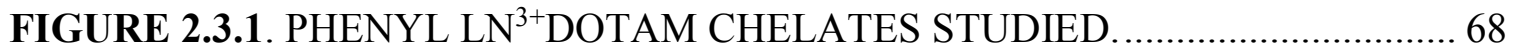




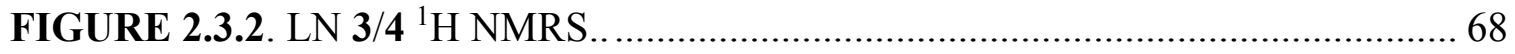

FIGURE 2.3.3. LN3/4 NMR FLOWCHART ....................................................... 70

FIGURE 2.3.4. YB4 VARIABLE TEMPERATURE EXPERIMENTAL DATA. ........ 71

FIGURE 2.3.5. EU3 VARIABLE TEMPERATURE DATA.................................... 72

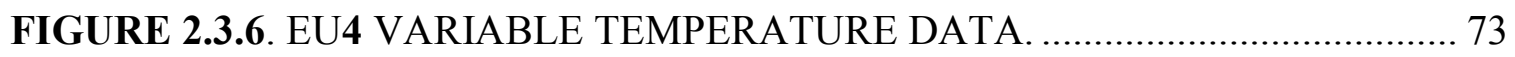

FIGURE 2.3.7. ZOOMED EU4 VARIABLE TEMPERATURE DATA...................... 74

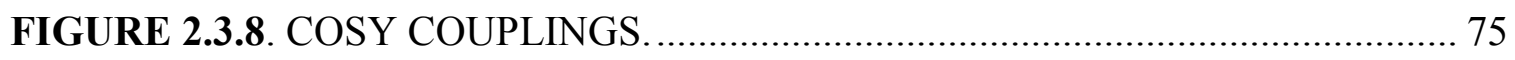

FIGURE 2.3.9. EU3/4 COSY SPECTRA. ........................................................... 76

FIGURE 2.3.10. SUBSTITUTED CARBON GAUCHE CONFORMATIONS. .......... 79

FIGURE 2.3.11. EU3/4 VARIABLE TEMPERATURE TRACES ............................... 80

FIGURE 2.3.12. TSAP AND SAP ISOMER RATIO TRENDS. ............................... 81

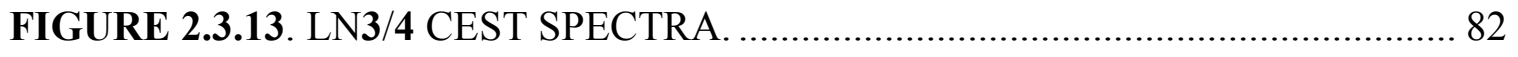

FIGURE 2.3.14. EU3 CEST SPECTRA AND OMEGA PLOT .................................. 83

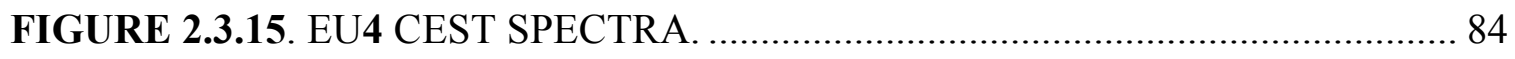

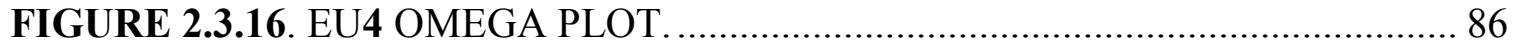

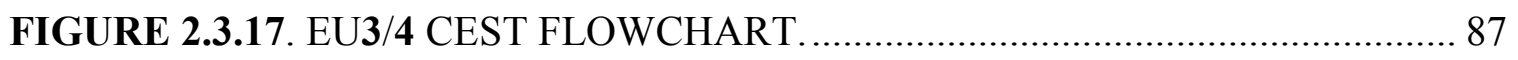

FIGURE 3.1.1. VESICLE STABILITY OVER 8 DAYS ......................................... 90

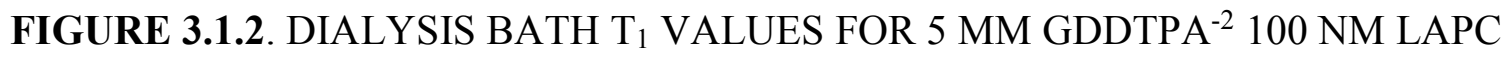

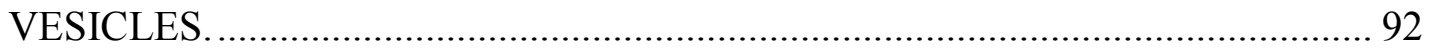

FIGURE 3.1.3. DIALYSIS BATH $\mathrm{T}_{1}$ VALUES FOR $5 \mathrm{MM} \mathrm{GDDTPA}^{-2} 100 \mathrm{NM}^{2} \mathrm{LPC}$

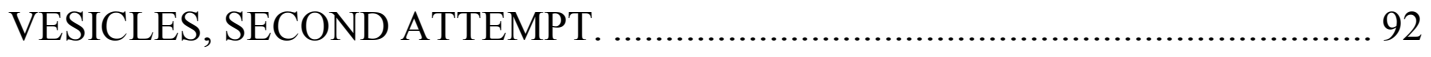

FIGURE 3.1.4. OSMOTIC SHRINKING MECHANISM......................................... 93 
FIGURE 3.1.5. OSMOTIC SWELLING MECHANISM. 94

FIGURE 3.1.6. MEAN VESICULAR DIAMETER BEFORE AND AFTER DIALIZATION. 94

FIGURE 3.2.1. VARIOUS CONCENTRATED SAMPLES OF GDDTPA ${ }^{-2}$ SHOWING THE CHANGING RATE OF T 1 RELAXATION. 96

FIGURE 3.2.2. $T_{1}$ VALUES OF VESICLE CONTAINING GDDTPA ${ }^{-2}$ SAMPLES

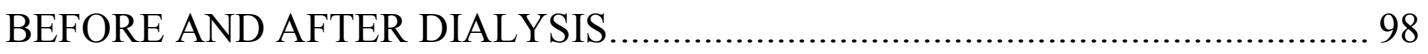

FIGURE 3.2.3. 5 MM GDDTPA ${ }^{-2}$ VESICLE SYSTEMS.......................................... 105

FIGURE 3.2.4. $5 \mathrm{MG}$ /ML DPPC PREPARED AT $43^{\circ} \mathrm{C}$, BEFORE AND AFTER EXTRUSION AT 50 AND 200 NM. 106

FIGURE 3.2.5. $5 \mathrm{MG}$ /ML DPPC+C PREPARED AT ROOM TEMPERATURE, BEFORE AND AFTER EXTRUSION AT 50 AND 200 NM............................... 106

FIGURE 3.2.6. SIZE COMPARISON $T_{1}$ VALUES FOR 0-5 MM GDDTPA ${ }^{-2}$ SAMPLES. 110

FIGURE 3.2.7. VESICLE CONCENTRATION COMPARISON $T_{1}$ VALUES FOR 0-5 MM GDDTPA $^{-2}$ SAMPLES 111

FIGURE 3.2.8. VESICLE CONCENTRATION COMPARISON $T_{1}$ VALUES FOR 15 MM GDDTPA ${ }^{-2}$ SAMPLES 112

FIGURE 3.2.9. FORMATION METHOD COMPARISON $T_{1}$ VALUES FOR 0-5 MM GDDTPA $^{-2}$ SAMPLES 113

FIGURE 3.2.10. FORMATION METHOD COMPARISON $\mathrm{T}_{1}$ VALUES FOR $15 \mathrm{MM}$ GDDTPA $^{-2}$ SAMPLES. 114 
FIGURE 3.2.11. DATA COLLECTION COMPARISON $T_{1}$ VALUES FOR 1-5 AND

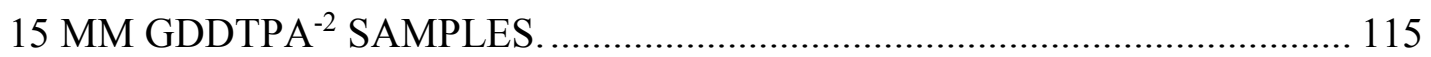

FIGURE 3.3.1. PRELIMINARY CEST SPECTRA ON VESICLE SAMPLES.......... 119 FIGURE 3.3.2. TMDTPA ${ }^{-2}$ CONCENTRATION SHIFT DEPENDENCE EXPERIMENT. 121

FIGURE 3.3.3. $300 \mathrm{MM}^{\text {TMDTPA }}{ }^{-2} 200$ NM LAPC VESICLE CEST SPECTRA.... 122

FIGURE 3.3.4. 300 MM YDTPA $^{-2} 200$ NM LAPC VESICLE CEST SPECTRA....... 122 FIGURE 3.3.5. 300 MM TMDTPA ${ }^{-2} 200$ NM LAPC $+C$ VESICLE CEST SPECTRA

FIGURE 3.3.6. $300 \mathrm{MM} \mathrm{YDTPA}^{-2} 200$ NM LAPC+C VESICLE CEST SPECTRA.. 123 FIGURE 3.3.7. CEST ASYMMETRIC ANALYSIS.............................................. 125

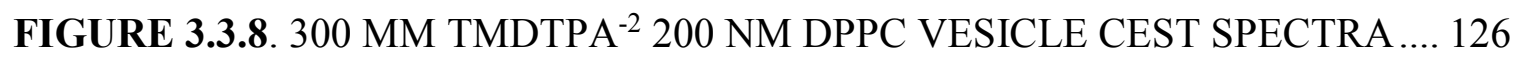
FIGURE 4.1.1. SYNTHETIC ROUTE TO LN-NB-DOTAM CHELATES. ................ 128 


\section{CHAPTER 1. INTRODUCTION OF MEDICAL IMAGING AND}

\section{MAGNETIC RESONANCE}

\subsection{The 123s of Medical Imaging}

\section{X-ray (1895)}

When the effects of X-rays were first observed by William Crookes and Johann Hittorf while working with Crookes tubes in the 1880 s, the scientists noticed that film plates that were kept near the tubes appeared fogged and had shadow artifacts despite the film plates being wrapped in paper and protected from light. The observed effect was not investigated and similar observations were made by many other scientists over the following years, including Ivan Pulyui, Fernando Sanford, Philipp Lenard, and Nikola Tesla. However, in 1895, Wilhelm Röntgen stumbled across the X-ray phenomenon while working with Crookes tubes and began studying the invisible rays methodically, calling them " $\mathrm{X}$ " radiation due to the type of radiation being unknown. He published the first medical X-ray of his wife's hand (Figure 1.1.1) shortly after and the name "X-ray" stuck with the technique. ${ }^{1}$

Figure 1.1.1. The first human X-ray performed. ${ }^{1}$ Wilhem Röntgen's wife's wedding ring is visible in the image.

In this type of medical imaging ${ }^{2}$, short X-ray pulses with varying photon energy levels are used to probe soft tissue in the body. The X-rays interact with the tissue of the body 
through two mechanisms. When the photons of the X-rays interact with an atom, the energy of the photon ejects an electron. The photon can either use all of its energy to eject the electron, in which case there are no scattering photons as a result of the interaction, or the photon does not use up all of its energy and the energy left over scatters the photon in a different direction with less energy, which can then interact with other atoms or can pass through the tissue to the detector or leave the body in any direction. The former process is known as the photoelectric effect and is the main source of contrast for this technique, while the latter is known as Compton scattering and is the main source of noise in radiographs. In both cases, the ejected electron is free to move around and ionize neighboring atoms, creating free radicals which can damage DNA and wreak havoc inside the patient's body. ${ }^{3-6}$

As the X-ray passes through the body, the photons interact with atoms and the energy of the photons decreases as they are absorbed. When these photons are absorbed and are no longer able to penetrate through tissue, they are not able to reach the detector and fewer photons are detected in the shadow of the structure absorbing the photons. Since higher energy photons are able to move more readily through tissue without being absorbed, the X-rays used need to be higher in energy (hard X-rays) as opposed to lower in energy (soft X-rays). In the body, most tissue is made up of $\mathrm{C}, \mathrm{H}, \mathrm{O}$, and $\mathrm{N}$ atoms. These atoms absorb lower energy photons and although the Compton scattering that occurs will show the presence of tissue, the tissue types are indiscernible. However, since calcium is present in high amounts in bones and teeth, this technique provides excellent contrast for the skeletal system. 
Calcium has a high atomic number which allows it to efficiently absorb higher energy X-ray photons without having any leftover scattering of the photons. This means that when the high energy photons come in contact with calcium in the bones and teeth, the photons are absorbed via the photoelectric effect and contrast is generated, while the photons that don't interact with the calcium will pass through the body and hit the detector without interacting with many other atoms. This allows X-rays to be used to identify the pathology of the skeletal system and cavities in the teeth. X-rays can also be implemented to probe lungs and the abdomen by relying on the fact that trapped gases within these symptoms appear clear on images due to their low absorption of X-rays in comparison with body tissue. Thus, X-rays are utilized to diagnose lung diseases like pneumonia, lung cancer, and pulmonary edema and to identify intestine obstructions. The contrast in the abdomen can be increased with the administration of radiocontrast agents, which contain iodine or barium, which are both higher atomic number elements and therefore absorb the high energy photons well. These radiocontrast agents are given intravenously in the case of iodine, or orally in the case of barium.

While this technique is incredibly useful in probing systems that are difficult to view, X-rays are classified as a carcinogen, and prolonged, repeated exposure to the radiation used in the technique can lead to an increase in cancer occurrence and developmental problems in areas that are exposed. ${ }^{7-10}$ As a result, strict protective measurements are in place for those collecting the X-rays as well as the patients, with most medical professionals not giving X-rays unless it is medically necessary and the benefit of having 
the image outweighs the risk of exposing the patient to radiation, no matter how small the dose is.

\section{Ultrasound (1941)}

After Pierre Curie discovered piezoelectricity in 1880, ultrasonic waves were able to be produced and detected and were primarily used in industry settings. The first ultrasonic echo imaging device was developed in 1940 by Floyd Firestone, and in 1941 the first ultrasonic echo imaging was performed on the ventricles of the brain (Figure 1.1.2)by brothers Karl Theo and Friedreich Dussik. ${ }^{11}$ In the late 1940s ultrasonic energy was used for medical purposes by Dr. George Ludwig and ultrasound as we know it today was first used medically to assess the thickness of bowel tissue by John Wild. ${ }^{12}$
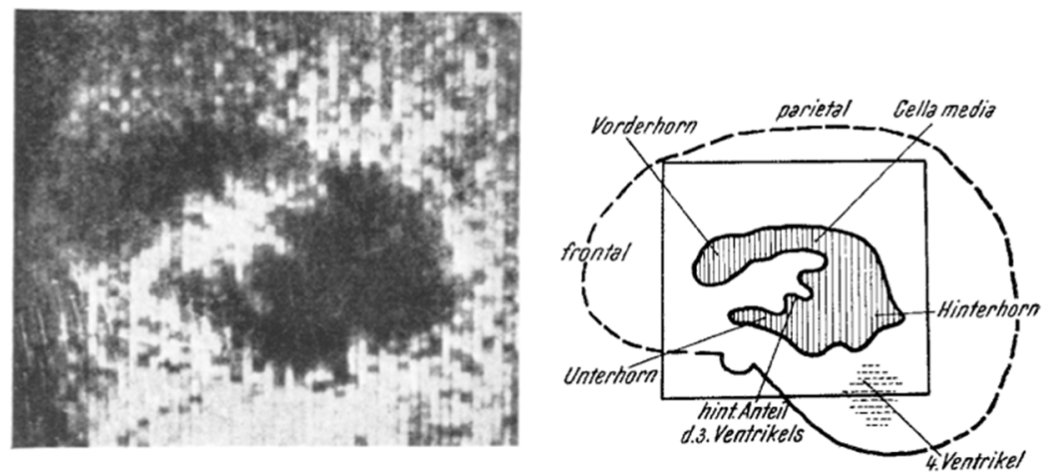

Figure 1.1.2. The first anatomic ultrasound performed. ${ }^{11}$ It was believed that the hyperphonogram could depict the ventricles when used on the head.

In this imaging technique ${ }^{13}$, high frequency broadband sound waves, much higher than humans can hear, are sent into the tissue and, depending on the tissue composition, will be reflected back to the probe at different time intervals and to varying degrees. The varying signal intensities and intervals of return can produce a 3D model in real time. The technique occurs in three different steps: producing a sound wave, receiving an echo, and interpreting the echoes. The sound waves used are ultrasonic and are produced with a piezoelectric 
transducer. Short electrical pulses are used to drive the transducer at desired frequencies. The frequency used depends on the application, but the waves produced are focused using an array of antennas that allow the direction and depth of the sound waves to be altered and focused as needed. The focusing of the sound waves produces an arc-shaped sound wave from the face of the transducer which then travels into the body and comes into focus at a desired depth.

Once the sound wave enters the body, it is reflected where the body has changes in acoustic impedance, like blood cells in plasma and small structures in organs. Once the sound wave reaches a change in impedance, it is scattered and reflected back in the general direction it came from. In this instance, the transducer, where the signal originated, becomes the detector. The same method by which the wave was created is used to detect the returning wave, but in reverse. Thus, the sound wave that returns to the transducer vibrates the transducer at its returning frequency and the vibrations are turned into electrical pulses that are transformed into the image seen on the screen of the ultrasonic scanner.

The scanner produces a digital picture by determining how long it took the echo to respond and how strong the returning echo was. With these two pieces of information, the scanner can piece together a greyscale image where strong signals are brighter, weak signals are darker, and the depth of the structure is determined by the amount of time it took the sound wave to return to the transducer. The image that results is a $2 \mathrm{D}$ image, however, 3D images can be produced by taking consecutive 2D images and digitally adding them together. 
Ultrasound imaging is utilized most in the diagnosis of diseases and visualization of structures in fetuses, internal organs, various joints, arteries and veins, and is used to guide placements in medical procedures. It is a quick, inexpensive, safe technique that allows good spatial resolution. However, the technique comes with limitations. The sound waves used in this technique cannot penetrate deep within tissues or pass through bone structures, so imaging certain deeply embedded areas or bone encompassed areas, such as the brain, is impossible. This also makes the technique difficult to implement on obese patients with thicker structures. As a result, this method of imaging is used frequently today as one of the first imaging modalities in non-threatening cases, and is more frequently used in rural areas and third world countries as the main source of imaging due to its ease and mobility.

\section{Positron Emission Tomography (PET) / Single-Photon Emission Computed Tomography (SPECT) (1961)}

In 1896, radioactivity was stumbled upon when Henri Becquerel had to postpone fluorescence experiments with salts and film plates due to overcast skies in Paris. When Becquerel was able to finally run his experiments, he found that the film plates that he had prepared days earlier had images on them despite being protected from light sources with light-proof coatings and being placed in drawers. The images only occurred with one specific salt and that salt was uranium. Becquerel concluded that the source was the salt fluorescing, even without an energy source. ${ }^{14}$ He continued studying the effect and caught the attention of Polish scientists Marie Curie and her husband Pierre Curie. Marie Curie coined the term "radioactivity" after observing that uranium was able to electrify the air around it. The Curies also discovered polonium and radium, which were both more 
radioactive than uranium, and gave future scientists methodologies for detecting radioactive elements.

The first use of radioactive elements to study the body happened in the 1910s when George Hevesy used radioactive lead to demonstrate the rate at which the human body digests food. Through the 1920 s, Hevesy continued to use naturally occurring radioactive lead to study the metabolic processes of plants and animals. ${ }^{15,16}$ Hevesy co-discovered hafnium with Dirk Coster in 1922 which lead to the first development of artificial radioactive elements. Irene and Frederic Joliot-Curie also discovered new artificial radioisotopes in 1934 and their discovery suggested that new elements could be formed and potentially made safe. Despite their discoveries, development of radioisotopes used for medical imaging was largely put on hold during The Second World War when nuclear energy was being studied and harnessed for use in weapons. When the Manhattan Project came to a close, scientists were looking for new applications of the nuclear reactions and the development of nuclear medicine and radiotracers took off until the first conventional use in the 1960s when the first cerebral PET scan (Figure 1.1.3) was run to determine localized sensory, motor, and mental functions with ${ }^{133} \mathrm{Xe}^{17}$
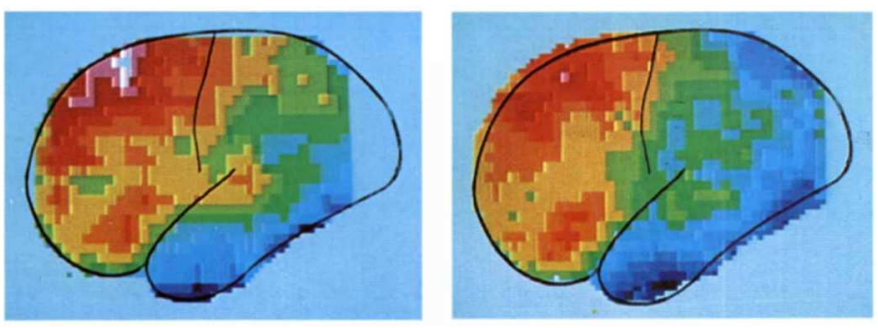

Figure 1.1.3. The first cerebral PET scan, performed in 1961 with ${ }^{133} \mathrm{Xe} .{ }^{17}$ This image represents a brain at rest.

The key to imaging with radioactive isotopes is the use of short lived radiolabeled tracers or radioisotopes, generally ranging from minutes to hours. The most commonly 
used tracers for imaging are compounds normally used by the body, either during metabolism or that bind to receptors or sites of drug action and have labelled atoms. Tracers are generally used to monitor and study biological processes and pathways of the molecules that are labelled. The most commonly used molecule today is fluorodeoxyglucose (FDG), which is an analog of glucose that is labelled with ${ }^{18} \mathrm{~F}$. Radioisotopes that are not labelled atoms as part of a molecule but rather simple soluble ions or part of radio-ligands, can also be used for imaging. Radio-ligands are preferred because they can transform normally nonspecific radioisotopes into targeted agents to specifically bind to certain types of tissue which causes the radioisotope to accumulate in desired areas of the body, thereby increasing the amount of signal seen in the image in that area. FDA-approved radioisotopes include the following: ${ }^{11} \mathrm{C},{ }^{14} \mathrm{C},{ }^{13} \mathrm{~N},{ }^{15} \mathrm{O},{ }^{18} \mathrm{~F},{ }^{67} \mathrm{Ga},{ }^{82} \mathrm{Rb},{ }^{89} \mathrm{Zr},{ }^{89} \mathrm{Sr},{ }^{90} \mathrm{Y},{ }^{99} \mathrm{Mo},{ }^{99} \mathrm{Tc},{ }^{111} \mathrm{In}$, ${ }^{123} \mathrm{I},{ }^{125} \mathrm{I},{ }^{131} \mathrm{I},{ }^{133} \mathrm{Xe},{ }^{153} \mathrm{Sm},{ }^{201} \mathrm{Tl}$, and ${ }^{223} \mathrm{Ra}$. Both labelled radiotracers and radioisotopes are injected into a patient and the natural decay process of the isotopes occurs inside the body while detectors monitor the radiation given off from the decay process in order to create images.

In PET imaging ${ }^{18}$, the isotopes in the bloodstream undergo positron emission decay (also known as positive beta decay) and each mechanism results in an emitted positron. These positrons travel through the tissue of the body until they collide with an electron, usually within a few millimeters of the decay process. When a positron and an electron collide, two gamma photons are released in opposite directions (at an angle of $180^{\circ}$ ). With the use of rotating gamma-detecting probes detecting in multiple directions concurrently, the location of each collision that occurs can be mapped and a 3D model can be 
produced/constructed using a computer. In SPECT imaging ${ }^{18}$, the isotope in the bloodstream emit gamma rays directly instead of from a secondary source where a positron and an electron collide. Though the mechanism of decay is different, the detection of the gamma radiation occurs in the same fashion with rotating gamma cameras and the same image reconstruction can occur using appropriate computer programs. However, due to the near-simultaneous detection of the two gamma rays produced in the positron emission mechanism, PET imaging has higher spatial resolution than SPECT imaging. Though they result in lower spatial resolution images, SPECT scans tend to cost less than PET scans and have the ability to utilize longer-lived, more easily obtained radioisotopes than PET.

Since the tracers are injected into the blood stream the tracers follow where the blood flows. This technique is often performed to identify tissue that have higher blood flow rates and therefore accelerated growth and metabolic rates, like cancer, metastasis, and infection. ${ }^{19-24}$ PET imaging has been used to assess the effectiveness of cancer therapies ${ }^{25-}$ ${ }^{27}$, psychiatric drug treatments ${ }^{28-32}$, and anti-atherosclerosis therapies ${ }^{33,34}$, to examine links between brain activity and psychological processes ${ }^{29,35-39}$, to study vascular diseases including stroke risk and inflammation responses ${ }^{40-45}$, and to look at musculoskeletal development ${ }^{46,47}$. SPECT imaging has been primarily used in functional cardiac and brain imaging. It has been used to diagnose ischemic heart disease based on the idea that there is decreased blood flow in the heart and therefore less signal from the heart would be seen in the images. ${ }^{48-50}$ With functional brain imaging, information on the cerebral blood flow, brain metabolism and energy use, and localized brain damage can be attained. ${ }^{51-54}$ 
While this is a powerful imaging technique, it exposes the patient to ionizing radiation, has low image resolution, and has serious cost and time limitations. Due to the imaging tracers decaying, they have to be synthesized and utilized relatively close together (depending on the half-life of the isotope, which ranges from minutes to hours), which lowers the ability to make and ship tracers to various areas. Also, the synthesis of radiotracers requires cyclotrons, which are expensive specialized instruments to buy and maintain.

\section{Computer Tomography (CT) (1971)}

In the early 1970s, the X-ray technique got an upgrade from limited direction 2D imaging to cross sectional 2D imaging and 3D models with the help of computers. The use of computers in medical imaging was well established by the 1970s with their use in PET and SPECT imaging, and the improvements being made in technology made the images created in the computer programs more promising than ever. By combining the X-ray technique, the PET scanner design, and the advances in technology and computer processing, Godfrey Hounsfield, an electrical engineer, developed the first CT scanner in London, England. In October of 1971, the first clinical CT scan was performed on a patient with a suspected brain tumor. ${ }^{55}$ The tumor was visible in an $80 \times 80$ matrix image (Figure 1.1.4) and the surgeon that excised the tumor is reported to have commented that "it looks exactly like the picture". It took two years for the first clinical scan to occur in the USA, but once it was shown how powerful and worthy of a tool it was, CT scanning grew in popularity and was further developed and the imaging quality improved over the next two decades. 


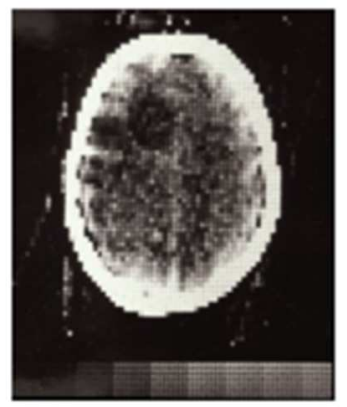

Figure 1.1.4. The first CT scan image of a brain tumor. ${ }^{55}$ The $80 \times 80$ matrix image took 5 minutes to acquire.

In X-ray computed axial tomography (CAT or CT) imaging, an X-ray source and radiation detectors are placed on opposing sides of a rotating platform. The patient is placed in the center of the instrument so that the body is between the radiation source and detectors. The platform rotates around the patient, collecting X-ray images in multiple directions and planes. The X-ray images are then combined using computer programs and, as a result, a 3D structural representation of the patient can be produced. In this technique, the use of aforementioned intravenously or orally administered x-ray radiocontrast agents help in the visualization of soft tissue and the abdomen, respectively. The use of computers for recreating models of the patient's anatomy allows for a more meaningful image to be produced when compared to conventional X-ray imaging alone.

CT imaging is mainly used as a preventative and screening technique, with bone injuries and lung/chest problems being best suited for non-contrast CT and cancer detection and tissue differentiation being best suited for contrast $\mathrm{CT}$, though images requiring contrast often are taken after images without contrast for pre- and post-contrast comparison. The increased imaging capability of this technique comes with a drawback in the form of 100 to 1000 times more radiation exposure than that of a conventional X-ray examination. ${ }^{8}$ Despite the radiation dose, CT imaging is used primarily in emergent 
situations due to the technique's fast scan time, good image resolution, and the ability to image bone structures, soft tissue, and the blood vessels simultaneously with the use of contrast.

\section{Magnetic Resonance Imaging (MRI) (1977)}

In 1937, Isidor Isaac Rabi discovered that an oscillating magnetic field could cause nuclei to flip their principal magnetic orientation and that the energy absorptions that occurred when resonance was achieved were detectable and different for distinct atoms. Rabi coined the phenomena he observed as "nuclear magnetic resonance". ${ }^{56-58}$ Edward Mills Purcell demonstrated similar absorption patterns and observed the NMR phenomenon while looking at solid paraffin inside an electromagnet with an oscillating current in $1945 .^{59-62}$ In 1946, instead of measuring absorption similar to Rabi and Purcell, Felix Bloch detected a nuclear induction signal from a small water sample in nearby receiver coils. All three scientists are given credit for the discovery of nuclear magnetic resonance (NMR), a technique frequently used in chemical analysis and the foundation for magnetic resonance imaging (MRI). ${ }^{63,64}$

Raymond Damadian distinguished normal healthy tissue from cancerous tissue in 1971 through NMR analysis. ${ }^{65}$ Damadian concluded that the two tissue types contain different amounts of water which lead to different hydrogen signals, with healthy tissue having less signal in the analysis because it has less water than tumor samples. Shortly after his observation, Damadian designed a machine for and applied for a patent for the use of NMR in distinguishing healthy versus diseased tissue in the human body. Using the same concepts as described by Damadian in his patent application, Paul Lauterbur produced the 
first NMR image of a test tube in $1973 .{ }^{66}$ After Damadian built the first MRI scanner with his graduate students, Damadian took the first MRI scan of a healthy human body in 1977 (Figure 1.1.5) and performed another MRI scan of a human body with cancer in $1978 .{ }^{67-70}$

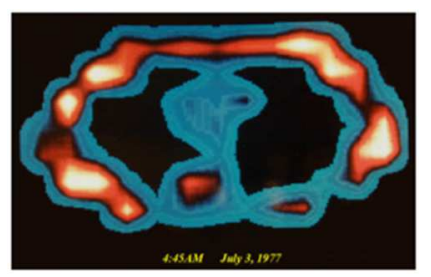

Figure 1.1.5. The first MRI image of Raymond Damadian's torso, around T7/T8, performed in $1977 .{ }^{68}$ The instrument was only able to produce one-dimensional images due to the instrument's design.

Magnetic resonance (MR) imaging is a technique that utilizes a strong magnet, combinations of radiofrequency (RF) pulses, and gradient magnetic fields to manipulate and detect the proton signal from water in the body, the most abundant nuclei in the human body at approximately $70 \%$. This signal is spatially encoded by detecting coils and the information is fed into a computer which can use the data collected, once processed, to create $2 \mathrm{D}$ slices of the anatomy which can be combined to make 3D models. MR imaging is best suited for soft tissue imaging due to its high water content; thus, the diagnosis of ligament, tendon, cartilage, spinal cord, and muscle injuries is easily achieved. Depending on the processing parameters, differentiation of tissue is possible by how much water is present in each tissue type. The ability to differentiate tissue types can be increased via a process called tissue weighting. If weighting the images does not provide sufficient contrast, contrast agents can be administered intravenously. Brain injuries, tumors, strokes, and any abnormality that results in a change in diffusion across membranes are readily apparent when contrast media is administered. ${ }^{71-76}$

The drawback with MR imaging is that the acquisition time is long. This technique requires compliant patients who lie still through the duration of the examination since any 
movement changes the spatial arrangement of water and will afflict the spatial encoding of the signal. Lying still for extended periods of time can be difficult with seriously ill patients, patients who suffer from claustrophobia, patients who suffer from dementia and related illnesses, and for patients who are required to lie in uncomfortable positions in order to get the best images. For this reason, MR imaging is not usually used in emergent situations, but is capable of producing detailed anatomical images with high resolution.

\section{Comparison of Techniques and Effectiveness}

When comparing the different major imaging techniques, each has its own set of benefits and drawbacks. X-ray images can diagnose skeletal injuries, ultrasound examinations can give real time information concerning function and structure with superficial structures and organs, PET/SPECT imaging provides blood flow dependent metabolic information, CT imaging provides structural information, and MR imaging can provide structural, metabolic/physiological, and blood flow dependent information. In order to gather all the meaningful information from imaging to effectively diagnose a patient, a combination of these imaging techniques is most advantageous to the patient and their health-care since one imaging technique cannot satisfy all of the possible imaging requirements.

However, X-ray, CT, and PET imaging all expose the patient to radiation, and ultrasound imaging has poorer resolution that is restricted to structures close to the surface of the body which means missing the deeper structures. While MR imaging has long scan times, as the technique gets more established and is further developed, the acquisition time may become comparable to that of CT imaging. When the first CT scan was taken, each 
scan run took about five minutes with a similar processing time for an $80 \times 80$ matrix image; currently, CT slices are acquired in less than 0.3 seconds and the images produced can have up to a 1024 x 1024 matrix. The advancement of technology and protocols may allow for the MRI technique to surpass CT and PET imaging times.

Despite its limitations, MRI provides anatomical, physiological, and blood flow dependent information from one imaging modality. In order to achieve the same level of information from imaging data, combining modalities would need to occur. This could be achieved by utilizing supplementary techniques, such as elastography, tactile imaging, photoacoustic imaging, thermography, and functional near-infrared spectroscopy (FNIR), in workups to enhance the aforementioned major imaging techniques. Co-registration of major imaging techniques to acquire anatomic and metabolic information in one examination is another current trend in medical imaging. PET-CT instruments have been increasingly common over previous years and PET-MRI instruments are starting to emerge as well. However, the combination of PET-CT gives the patient two doses of radiation in a short amount of time, and the combination of PET-MRI is not necessary since both anatomical and metabolic/physiological information can be obtained from an MRI exam alone which renders the PET exam pointless.

\subsection{Nuclear Magnetic Resonance (NMR) and Magnetic Resonance Imaging (MRI)}

\section{Spin Dynamics and NMR Theory}

Most nuclei have randomly oriented spins that are associated with a small magnetic moment $(I=+/-1 / 2)$. In the earth's magnetic field, which is relatively weak, these spins are randomly oriented in space (Figure 1.2.1). However, when these nuclei are placed into a 
strong external magnet, such as that of an MRI, the spins loosely orient themselves either with or against the applied magnetic field (Figure 1.2.1), $\mathrm{B}_{0}$, according to the Boltzmann distribution.
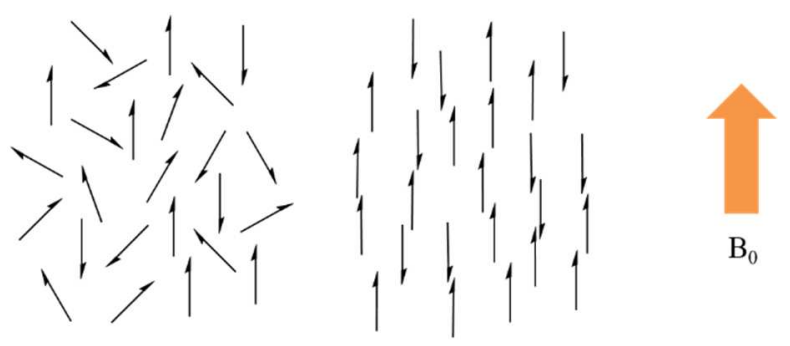

Figure 1.2.1. Spins in a weak magnetic field and a strong magnetic field. Individual spins randomly oriented in a weak magnetic field (left) and spins aligning with and against a strong magnetic field, $\mathrm{B}_{0}$.

The spins aligned with $\mathrm{B}_{0}$ are distributed in the low energy state, $\alpha(+1 / 2)$, and the spins aligned against $\mathrm{B}_{0}$ are distributed in the high energy state, $\beta(-1 / 2)$. The population of spins in these two states is nearly equal with a small excess populating the lower energy state. As $B_{0}$ is increased in strength, the energy difference between the $\alpha$ and $\beta$ energy states increases and the number of spins in excess in the $\alpha$ energy state increases (Figure 1.2.2).

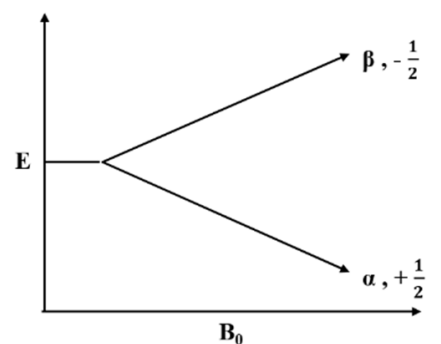

Figure 1.2.2. The Boltzmann distribution, depicting the energy difference between the $\alpha$ and $\beta$ energy states and the increasing difference between them with an increasing magnetic field.

The slight excess of spins in the $\alpha$-energy state per million spins in the body creates a small magnetic moment (Figure 1.2.3), $\mathrm{M}_{0}$, aligned parallel to the external magnetic field, $\mathrm{B}_{0}$. The magnitude of the magnetic moment, $\mathrm{M}_{0}$, is directly proportional to the strength of the magnetic field and is significantly less than $\mathrm{B}_{0}$ and therefore cannot be detected while aligned with the applied magnetic field. 


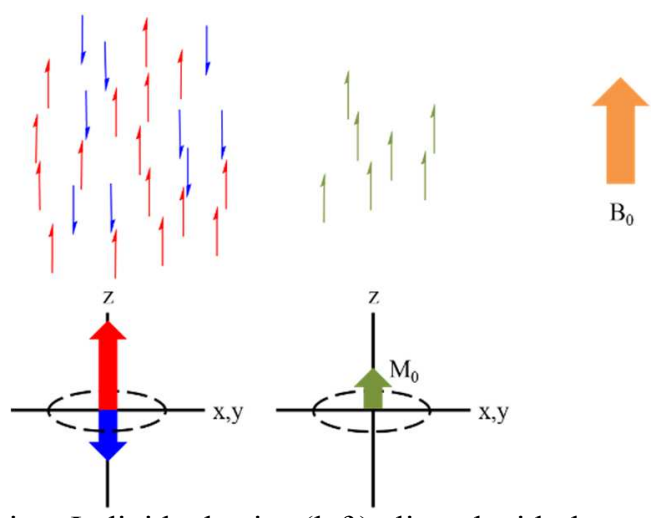

Figure 1.2.3. Net magnetization. Individual spins (left) aligned with the magnetic field (red) and aligned against the magnetic field (blue). The handful of individual spins (right) that make up the net magnetization signal (green). The vectors on the axes indicate the net magnetizations for each part (red and blue) and the overall net magnetization for the system (green).

To measure the net magnetization, $\mathrm{M}_{0}$, created by the difference of populations in the spin states, the system is perturbed using low energy radiofrequency pulses. These RF pulses, or $\mathrm{B}_{1}$, cause the net magnetization, $\mathrm{M}_{0}$, to end up in the $\mathrm{x}-\mathrm{y}$ plane where the signal, $\mathrm{M}_{\mathrm{x}-\mathrm{y}}$, can be detected. However, when the spins are pulsed into the $\mathrm{x}-\mathrm{y}$ plane, they flip perpendicular to both $\mathrm{B}_{0}$ and $\mathrm{B}_{1}$ and precess in unison according to the Larmor frequency (Figure 1.2.4).
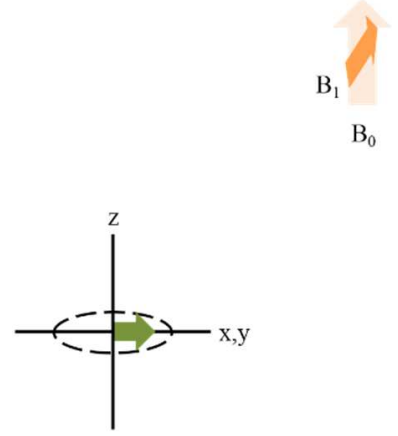

Figure 1.2.4. Net magnetization in the $x-y$ plane after an RF pulse, $B_{1}$.

As soon as the spins have been flipped into the $\mathrm{x}-\mathrm{y}$ plane, and once the radiofrequency pulse $\mathrm{B}_{1}$ has been turned off, the spins simultaneously begin to relax back to their original distribution in $\mathrm{B}_{0}$ and lose the coherency that is achieved with $\mathrm{B}_{1}$ (Figure 1.2.5). 

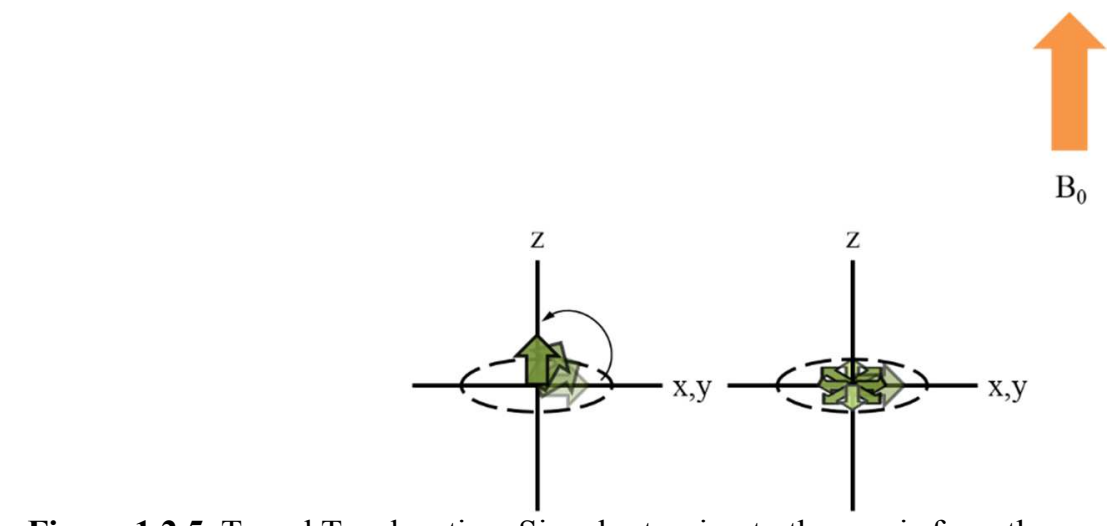

Figure 1.2.5. $T_{1}$ and $T_{2}$ relaxation. Signal returning to the $z$-axis from the $x-y$ plane in $T_{1}$ relaxation (left) and signal losing coherency in the $x-y$ plane in $T_{2}$ relaxation (right).

The former process is longitudinal or spin-lattice relaxation $\left(\mathrm{T}_{1}\right)$ and the latter is transverse or spin-spin relaxation $\left(\mathrm{T}_{2}\right)$. Depending on the type of tissue being imaged, relaxation rate constants will differ and the rate at which signal is lost in the x-y plane will change. The differences in both the spin density being manipulated and the relaxation times of those spins is the basis for spatial 3D imaging. Various RF pulse sequences can also be applied based on the type of image being taken. In typical MRI exams, differences in water content are looked at due to the human body being comprised of nearly $70 \%$ water and there being two hydrogen protons for every one water molecule. Other nuclei that can be probed for diagnostic imaging include ${ }^{13} \mathrm{C},{ }^{15} \mathrm{~N},{ }^{17} \mathrm{O},{ }^{19} \mathrm{~F},{ }^{23} \mathrm{Na}$, and ${ }^{31} \mathrm{P}$.

\section{MRI Theory}

MR images are weighted to increase contrast between tissue types using different pulse sequences. There are three predominant imaging types (Figure 1.2.6): $\mathrm{T}_{1}$-weighted $\left(\mathrm{T}_{1} \mathrm{~W}\right)$, $\mathrm{T}_{2}$-weighted $\left(\mathrm{T}_{2} \mathrm{~W}\right)$, and proton density $(\mathrm{PD})$. 


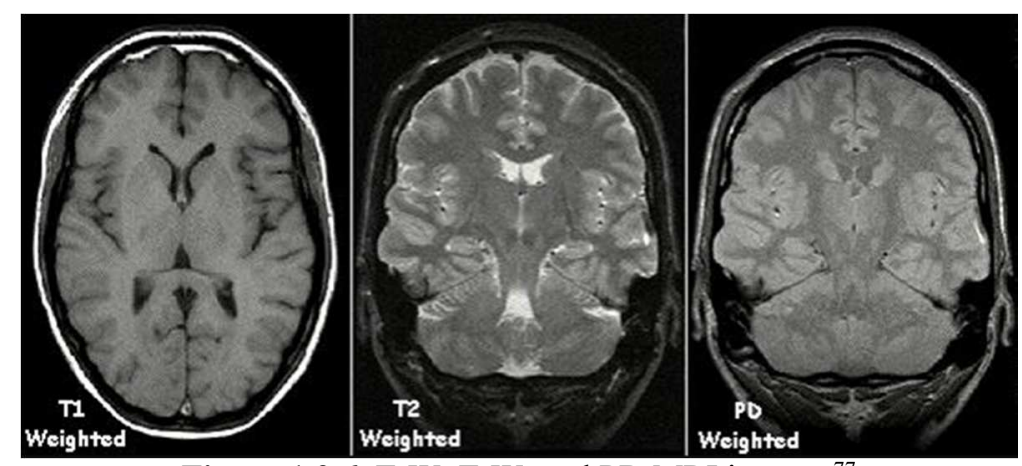

Figure 1.2.6. $\mathrm{T}_{1} \mathrm{~W}, \mathrm{~T}_{2} \mathrm{~W}$, and PD MRI images. ${ }^{7}$

In $\mathrm{T}_{1}$-weighted image collection, the fact that different tissues have different $T_{1}$ relaxation rates is taken advantage of. Once spins are pulsed into the transverse plane, the spins realign to their natural equilibrium state along $\mathrm{B}_{0}$. However, some tissue types return to equilibrium quicker than other tissue types. The faster a tissue type's spins return to alignment along $\mathrm{B}_{0}$, the more signal that tissue type has when pulsed to the transverse plane. If a tissue type's spins return to alignment slowly, not all the spins will be in phase when repulsed to the transverse plane, and thus that tissue type will have a weaker signal (Figure 1.2.7).

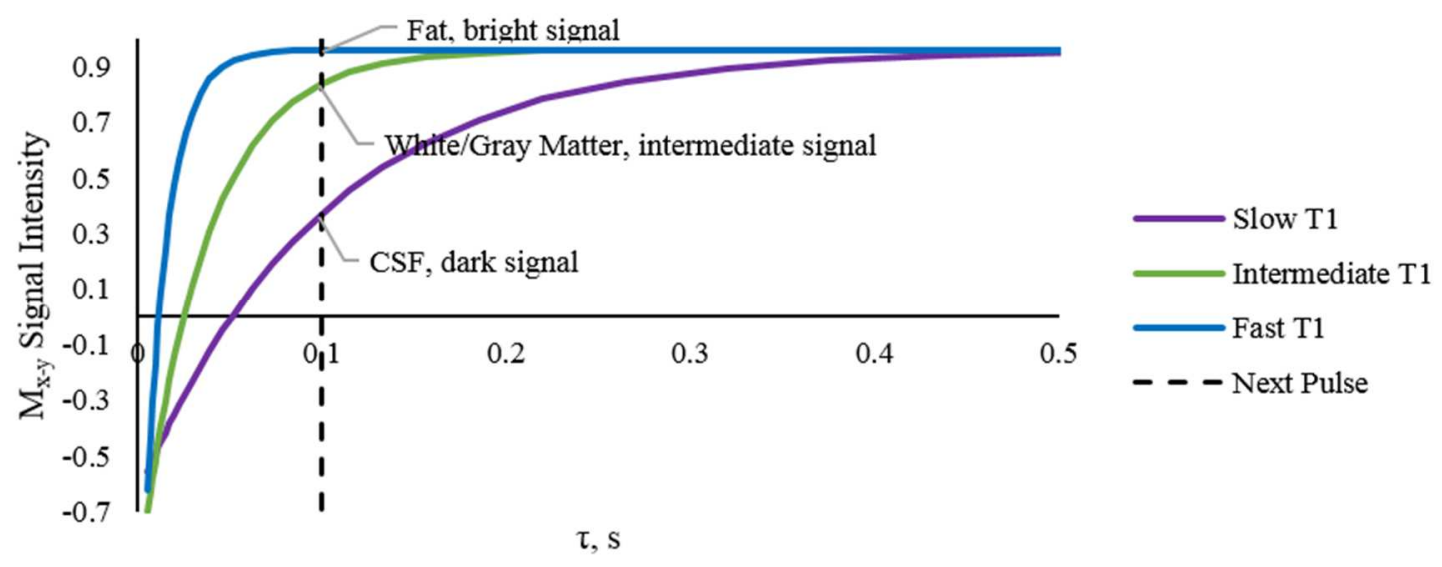

Figure 1.2.7. $T_{1}$ recovery curves. Fast (blue), intermediate (green), and slow (purple) $T_{1}$ recovery curves, shown with an example of where a new scan pulse would occur. In $\mathrm{T}_{1}$ weighting, the signal intensity at the time of the pulse is the signal that is kept and the rest of the signal is disregarded. 
This particular signal collection results in fat, protein-rich fluid, and slowly flowing blood to appear brighter, while bone, air, and higher water content areas appear darker. ${ }^{78}$ In $\mathrm{T}_{2}$-weighted image collection, the fact that different types of tissue have different $\mathrm{T}_{2}$ relaxation rates is taken advantage of. Once spins are pulsed into the transverse plane, the spins begin to lose the coherence that was achieved with the RF pulse $\mathrm{B}_{1}$. Some tissue type's spins precess around $\mathrm{B}_{0}$ faster than the Larmor frequency and some tissue type's spins precess slower. The differences in precessing speeds creates a "smearing out" of the signals, which causes the signal in the $\mathrm{M}_{\mathrm{x}-\mathrm{y}}$ plane to decrease (Figure 1.2.8).

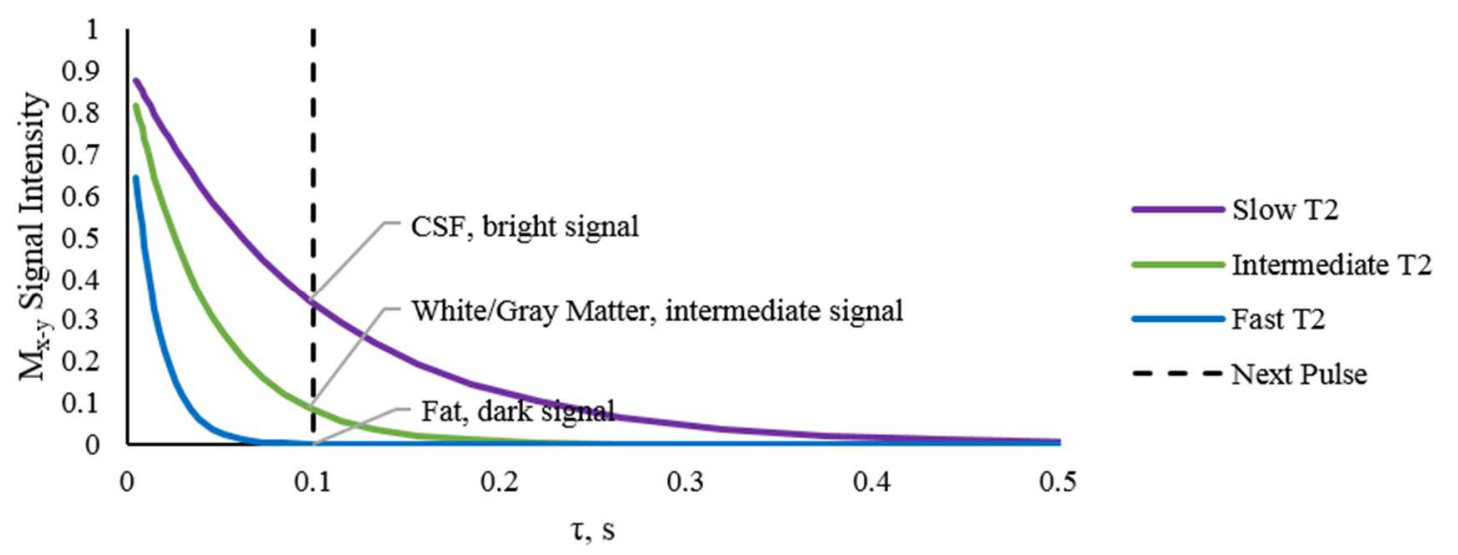

Figure 1.2.8. $T_{2}$ decay curves. Fast (blue), intermediate (green), and slow (purple) $T_{2}$ decay curves, shown with an example of where a new scan pulse would occur. In $\mathrm{T}_{2}$ weighting, the signal intensity at the time of the pulse is the signal that is kept and the rest of the signal is disregarded.

This signal collection results in higher water content areas appearing brighter, while bone, air, fat, and protein-rich fluid appear darker. ${ }^{78}$ Proton density (PD) weighted images combine features from both the $\mathrm{T}_{1}$ - and $\mathrm{T}_{2}$-weighted imaging techniques. The tissues that have the highest density of protons appear the brightest in this imaging technique, so waterrich tissues and fluids in the body will appear bright and moving blood, bone, and air will appear dark. ${ }^{78}$ This type of imaging gives high signal distinction between cartilage and fluid, which makes it highly useful in the imaging of joints. The appearance differences 
inherent in the different tissue types make diagnosis of anatomical abnormalities facile. The inherent differences in relaxation rates and water densities between tissues do not always produce enough contrast to see abnormalities. In order to differentiate between various lesions in the body, an intravenous contrast agent can be administered to create contrast in the images ${ }^{79-82}$.

In MRI, $T_{1}$ contrast agents are utilized to increase the difference in $T_{1}$ relaxation rates by shortening the $T_{1}$ rate constant of water protons directly in the vicinity of the contrast agent. ${ }^{83,84}$ The contrast agent is injected intravenously into the patient and travels through the body via the circulatory system, where it nonspecifically distributes in the intercellular space in tissues where it can diffuse through the vasculature. In cases where the diffusion rate through the vasculature is changed, like in malignant tissues with leaky vasculature or in spots where there are injuries to the vasculature, the contrast agent will be able to move further into the tissue/area and will affect water that is normally further away from the effects of the contrast agent. In these cases, physical abnormalities become readily apparent in the $T_{1}$-weighted images due to the differences in the $T_{1}$ relaxation rates. $\mathrm{Gd}^{3+}$ is a particularly good $T_{1}$ relaxation metal due to its high isotropic paramagnetism, a consequence of the 7 unpaired f-shell electrons in the nucleus. Clinically approved $\mathrm{T}_{1}$ contrast agents are derivatives of GdDOTA ${ }^{-1}$ and GdDTPA ${ }^{-2}$ chelates (Figure 1.2.9) and, as such, their function is closely tied to the chemistry of the rare earth lanthanides. 


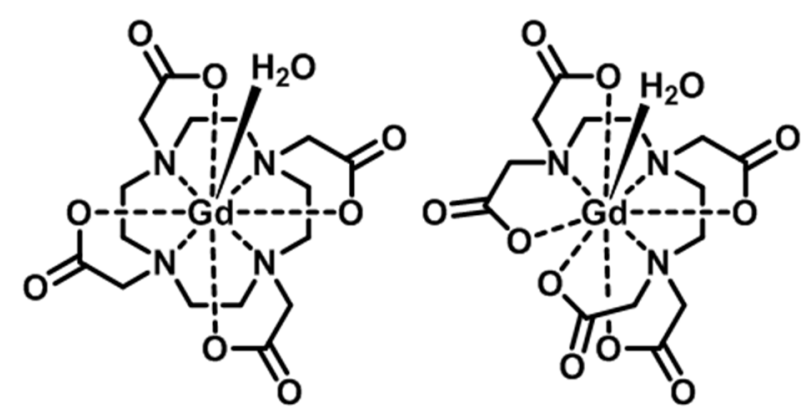

Figure 1.2.9. Chelate structures. GdDOTA ${ }^{-1}$ (left) and $\mathrm{GdDTPA}^{-2}$ (right) structures.

Another MRI technique has gained popularity more recently: chemical exchange saturation transfer (CEST) imaging. ${ }^{85}$ This imaging modality relies on the detection of exchange rates for information. This technique offers many advantages over conventional MRI exams with or without the use of contrast agents. With CEST imaging, contrast can be turned off if needed, ratiometric imaging is possible ${ }^{86,87}$, and physiological information such as $\mathrm{pH}^{88,89}$, temperature ${ }^{88,90}$, and metabolic information ${ }^{91-93}$ can be ascertained. This type of imaging allows for more information to be gathered during an exam than conventional MRI exams.

In systems where chemical exchange is present, instead of using $90^{\circ}$ radiofrequency pulses to manipulate spin orientations, low power saturation pulses are used to manipulate the spins that exist in two, or more, exchanging pools. In an exchange system of two pools, pool A and pool B, if a saturation pulse is applied to pool B the saturation pulse equalizes the populations in the $\alpha$ and $\beta$ states which then causes no net magnetization to be present for pool B (Figure 1.2.10). 

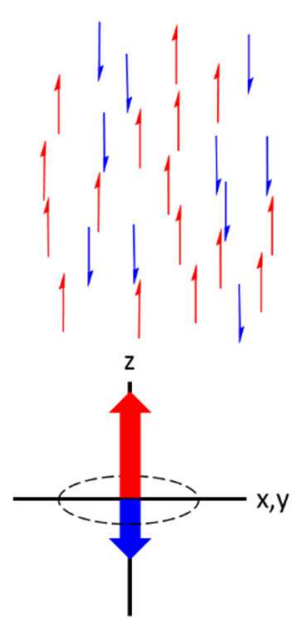
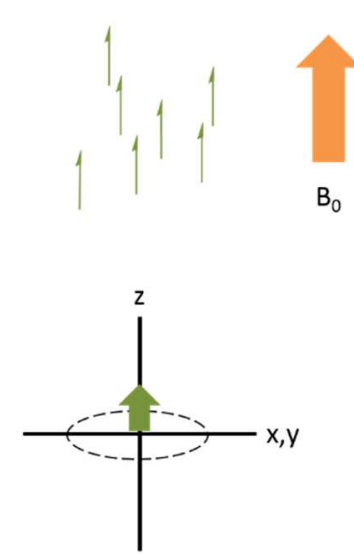
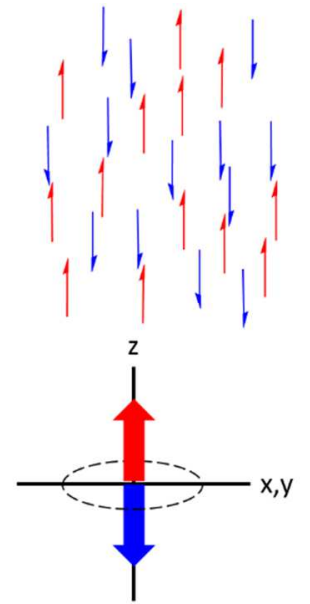
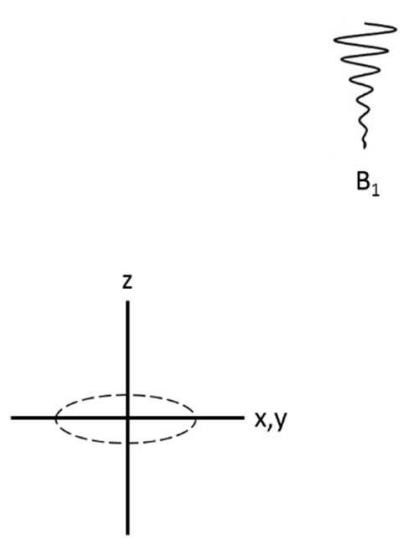

Figure 1.2.10. Saturation mechanism. The non-saturated system (left) and the saturated system (right) after a long, low power RF pulse (squiggly line) is applied to the system.

The two exchanging pools undergo chemical exchange and the equalized spins move from a saturated system, pool B, to a non-saturated system, pool A, which lowers the net magnetization in the non-saturated pool, pool A. Pool B, which still has a saturation pulse applied to it, then receives non-equalized spins from pool A and the process of saturation occurs again (Figure 1.2.11).

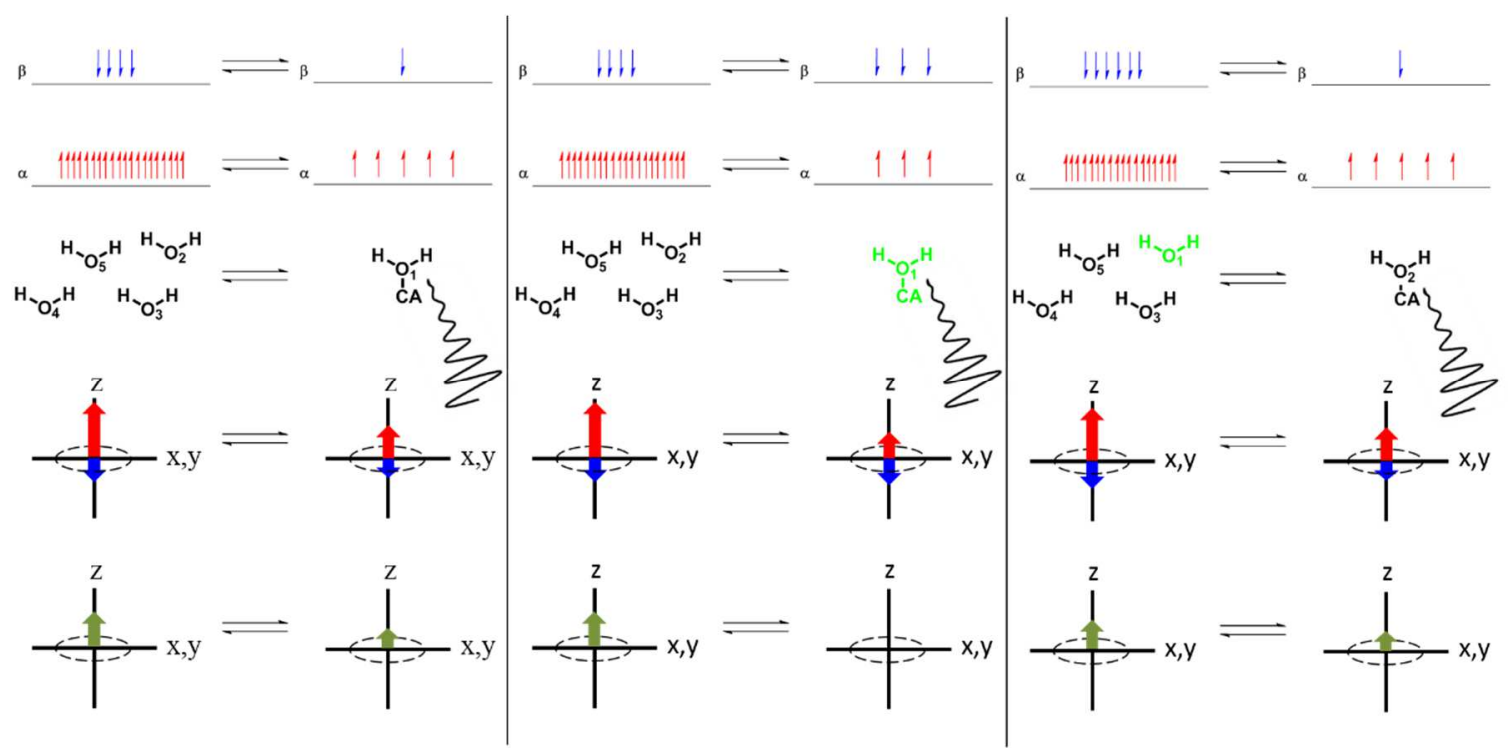

Figure 1.2.11. CEST mechanism. In all 3 panels: The left side represents the non-saturated bulk pool A and the right side represents the smaller saturated pool B. The top of each panel depicts the individual spins associated with each pool in their respective energy states, the center of each panel depicts the actual pools and shows pool B having the long, low power pulse being applied to it (the affected small molecule appears green for clarification), and the bottom of each panel depicts the net spins aligned with (red) and against 
(blue) the applied magnetic field, along with the overall net magnetization (green) of each pool. Left panel: The two pools just before saturation is applied. Center panel: Saturation in pool B is achieved. Right panel: The saturated water molecule exchanges from pool B to pool A, transferring its saturated individual spins, and decreasing the overall net magnetization of the bulk pool A. A non-saturated water molecule exchanges from pool A to pool B, with non-saturated individual spins, to be saturated.

As the process repeats itself the magnetization in pool $\mathrm{A}$ is monitored and the change in magnetization over time can provide information on parameters that affect exchange rates, like $\mathrm{pH}$, metabolism, temperature, and protein levels. This type of imaging requires a slow exchange rate to allow for spins to be sufficiently saturated but if the exchange rate is too slow, the magnetization representing pool A will not change a considerable amount to be seen in the images taken.

The type of exchange that is monitored can be either proton exchange or small molecule exchange. Both endogenous CEST agents, or agents that occur naturally in the body without needing to be injected, and exogenous CEST agents, or agents that are injected into a patient, can exhibit simple proton exchange from amine, amide, and alcohol moieties (Figure 1.2.12), and the frequencies associated with these protons (pool B) is within 10 ppm of the bulk water peak (pool A) and their signals are often buried under the water peak in vivo. 
A.

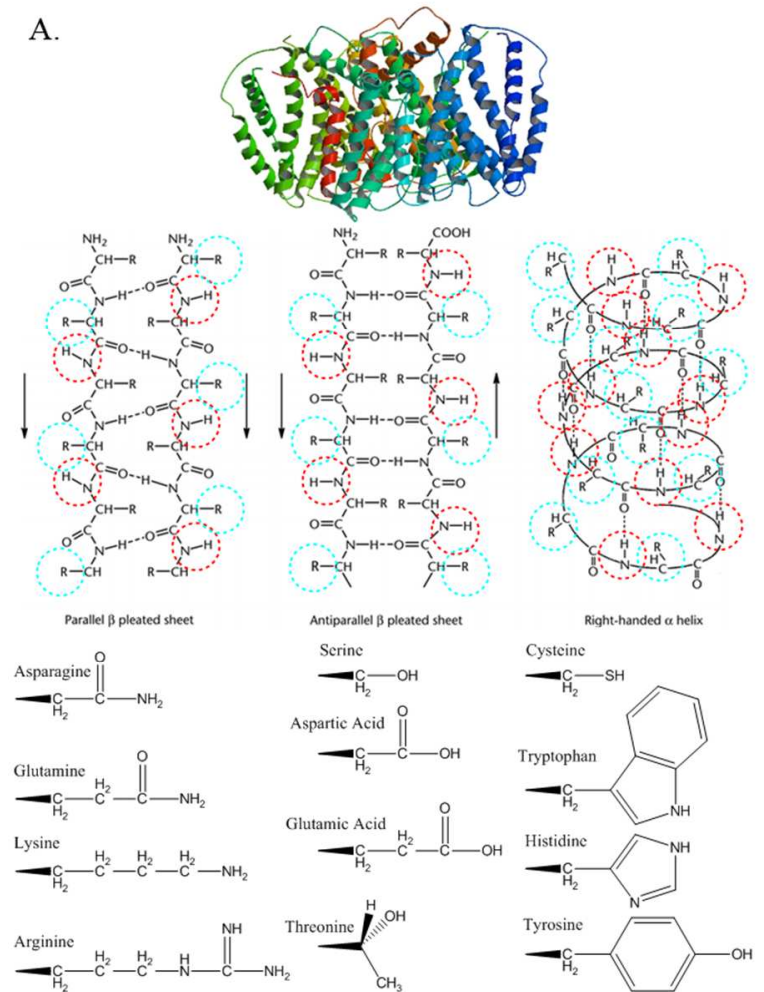

B.

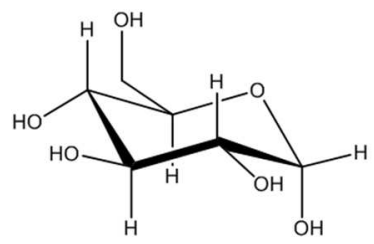

C.

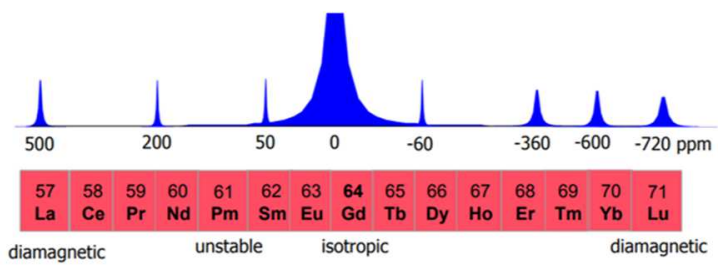

D.

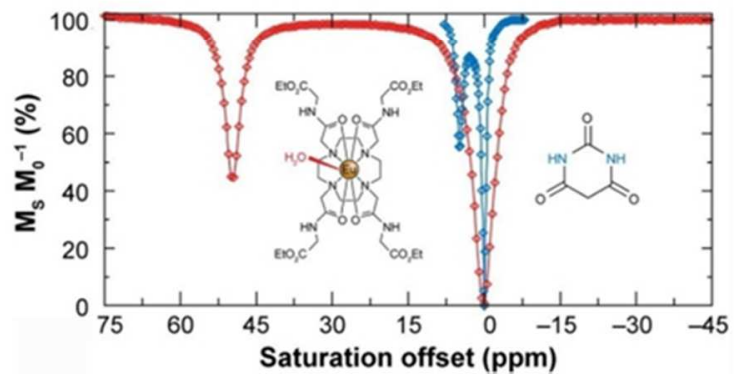

Figure 1.2.12. Sources of CEST imaging. A. Endogenous CEST source. Top: Protein tertiary structure. Center: Protein secondary structure showing exchangeable protons (amides are red circles, R groups are blue circles) ${ }^{94}$ Bottom: Amino acid R groups with exchangeable protons. B. Small molecule endogenous CEST source. Glucose, a common endogenous CEST agent used particularly in glucoCEST imaging. C. Lanthanide series and the shifting capabilities of paramagnetic $\mathrm{Ln}^{3+}$ ions. ${ }^{95} \mathrm{D}$. Paramagnetic CEST agent (red) vs. diamagnetic CEST agent (blue) ${ }^{96}$

Exogenous CEST agents can also exhibit whole small molecule exchange (like water), or a combination of whole small molecule and simple proton exchange mechanisms. The frequencies of the exchanging protons and small molecules on the exogenous CEST agents are also within $10 \mathrm{ppm}$ of the bulk water, unless a shift reagent, or a paraCEST agent, is used. A paraCEST shift reagent is an anisotropic paramagnetic material that causes a shift in signal for the species around it (Figure 1.2.12). If a paraCEST agent is used, the resonance of the protons or associated small molecules can be shifted up to several hundred ppm depending on which metal is used in the agent. If an exchanging proton is located on a ligand coordinated to the metal, it will be shifted a certain amount due to the reduced 
shielding from the metal. If a small molecule coordinated to the metal is exchanging, it will be shifted further down- or up-field due to the fact that it is in closer proximity to the metal.

With paraCEST agents, the increase in shift between the two pools allows for an increase in water exchange rate for the contrast agent used ${ }^{97-99}$; however the water exchange rate still needs to be slow. If the water exchange rate is too fast, the bound water cannot be fully saturated and the observed CEST effect decreases. Furthermore, in order for paraCEST imaging to be clinically applicable, the saturation pulses used need to be low power. At lower power pulse levels, the maximum amount of CEST effect observed occurs at slower water exchange rates (Figure 1.2.13). ${ }^{100}$

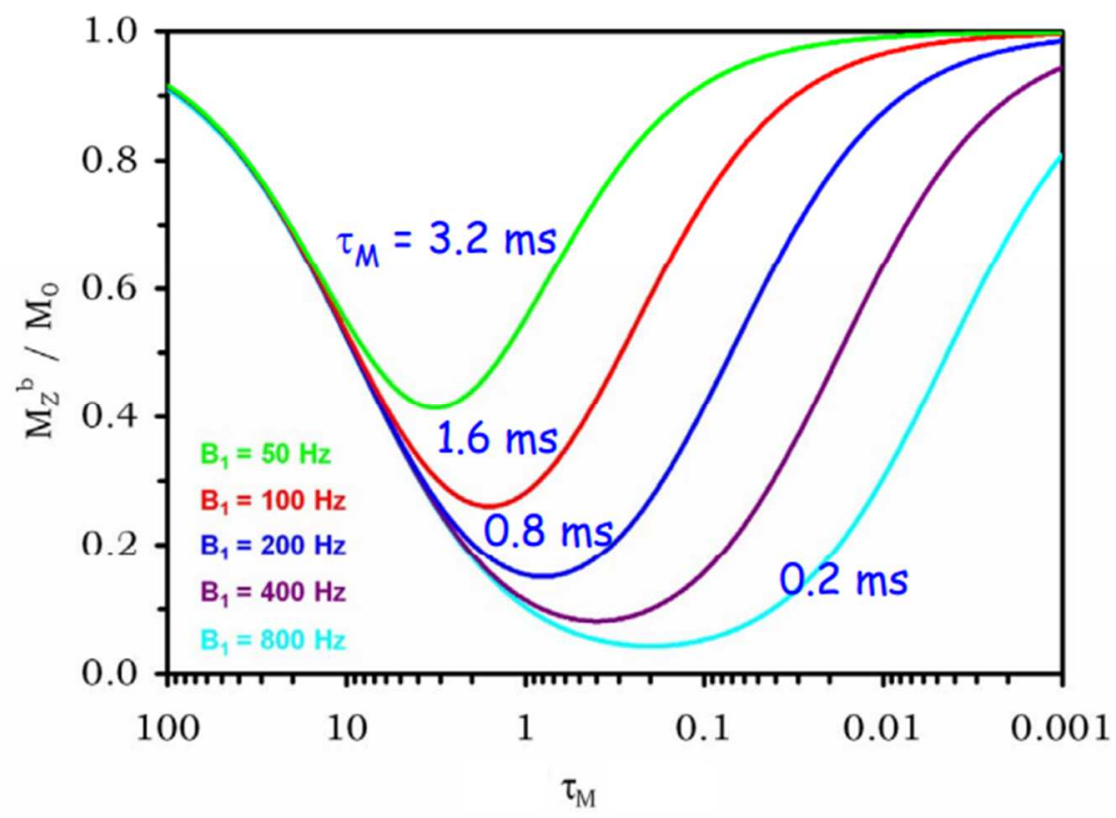

Figure 1.2.13. Water exchange rate vs. maximum CEST effect achieved at various saturation pulse power levels. ${ }^{101}$

Therefore, controlling water exchange rates in MRI contrast agents and slowing it enough to see CEST contrast is an important goal. Common types of paraCEST agents used include DOTAM chelates and lipoCEST agents (Figure 1.2.14). 


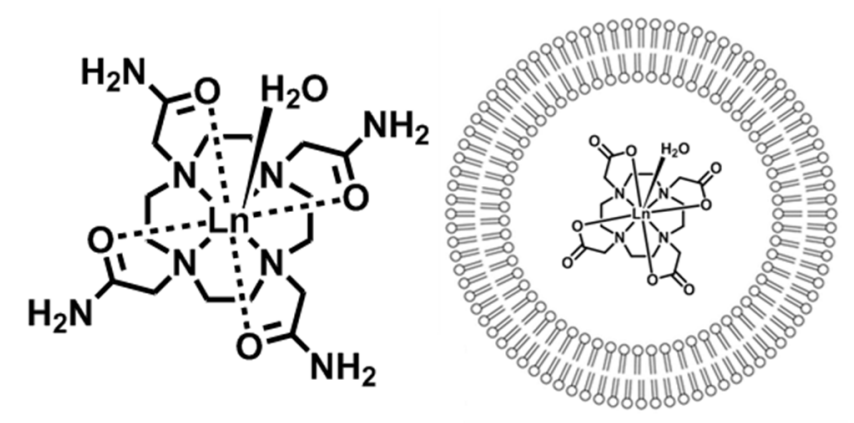

Figure 1.2.14. CEST agent structure. LnDOTAM ${ }^{+3}$ structure (left) and lipoCEST agent (right).

DOTA-tetraamide (DOTAM) chelates are DOTA-like chelates with amides instead of carboxylic acid moieties to coordinate to the lanthanide metal of choosing. Due to the changes in the coordinating atoms and atoms connected to the coordinating atoms, this derivative has slightly different chemical properties than DOTA chelates. DOTAM chelates exhibit slower water exchange rates than DOTA chelates. ${ }^{97,102}$ This is because the amide ligand that is being coordinated to the lanthanide ion is a poor electron donor, where in DOTA chelates the donating ligand is a carboxylic acid. The lanthanide ion, in the presence of a DOTAM ligand, is considered electron poor and requires more electron density from the coordinating water molecules; this causes the DOTAM chelate to have slower water exchange rates as the lanthanide ion holds on tighter to the coordinating water molecules. Utilizing DOTAM chelates allows a naturally slow water exchange rate and any changes made to the structure have the potential to further slow or speed up water exchange. The use of lipoCEST agents involves trapping water inside a phospholipid bilayer vesicle with a shift reagent and making the water inside chemically different from the bulk water outside the vesicle. ${ }^{103-106}$ The water exchange rate in the latter case is controlled by the type of lipid used to create the bilayer. In either case, the water exchange 
rate is slowed enough for the proper amount of saturation to occur and to be transferred, and CEST contrast is able to be detected.

\subsection{Contrast Agent Structure and Function}

\section{Lanthanides and Gadolinium Chemistry}

Although the lanthanides are in a group, each has unique electrochemical properties. The $4 \mathrm{f}$ orbitals are small and buried in the core of the lanthanide ion and, as such, they are poor at shielding the nucleus and rarely interact with ligand orbitals. Due to the poor shielding of the $4 \mathrm{f}$ electrons there is a gradual increase of attraction between the positively charged nucleus and the negatively charged electrons with each new proton and electron added across the series. The attraction causes the phenomenon known as the lanthanide contraction (Figure 1.3.1).

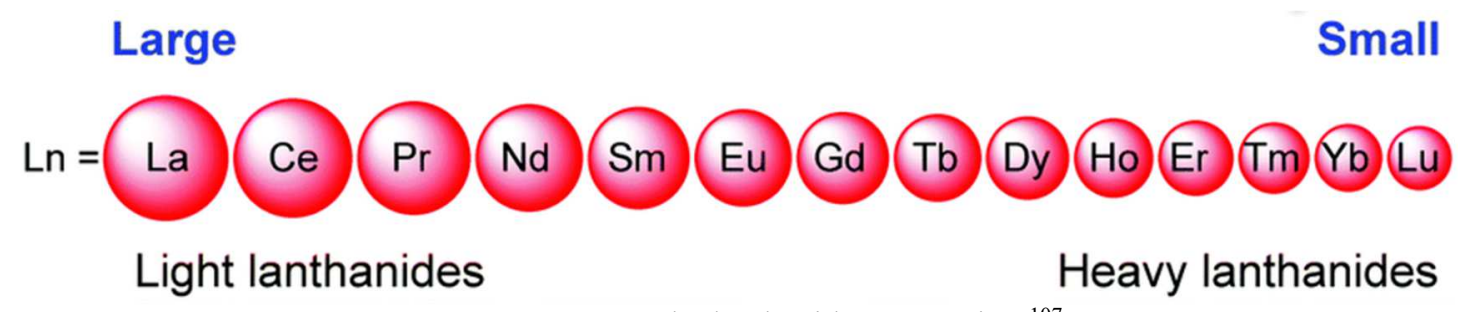

Figure 1.3.1. The lanthanide contraction. ${ }^{107}$

Lanthanide ions, across the series, are mainly found in the $3^{+}$oxidation state. $2^{+}$and $4^{+}$ states are possible but unlikely since they are unstable due to the reduction potentials between oxidation states being high and the ionization energies also increasing dramatically after the third one.

Gadolinium, found in the middle of the lanthanide series, holds particular interest for use in medical imaging because it is especially stable in the $3^{+}$oxidation state due to the half-filled 5d and 4f orbital shells (Figure 1.3.2). 


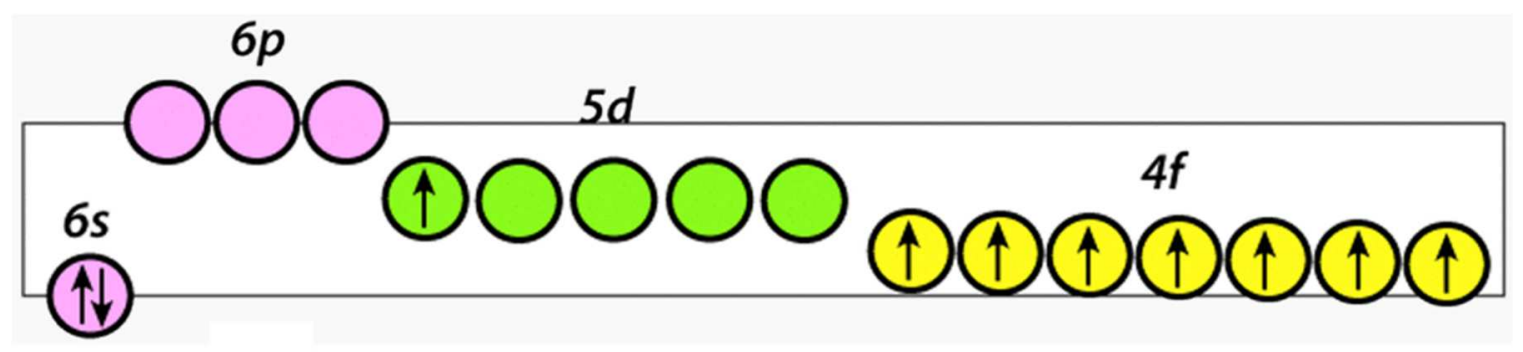

Figure 1.3.2. Electron configuration of $\mathrm{Gd}^{+3} \cdot{ }^{108}$

Due to the half-filled $4 \mathrm{f}$ orbital shell, gadolinium is a isotropic paramagnetic metal ion, meaning that it has a long electronic relaxation time ${ }^{109}$ and, when used in MRI, shortens the $T_{1}$ relaxation times of nearby water molecule protons which enhances the signal of affected water molecules near the metal. ${ }^{110}$ However, $\mathrm{Gd}^{3+}$ on its own is highly insoluble at physiological $\mathrm{pH}$ and temperature and is toxic because it is approximately the same size as $\mathrm{Ca}^{2+} .111$ Therefore it needs to be injected into the body as a chelate rather than as a free ion so it does not cause circulation problems or get distributed into the bone matrix. However, choosing an appropriate ligand requires careful thought into the coordination chemistry of both $\mathrm{Gd}^{3+}$, the coordination chemistry of the ligand, and the desired purpose of the contrast agent.

\section{Ligand Design and Chelate Structure}

All clinically approved contrast agents are derivatives of two contrast agents that contain polyaminocarboxylate ligands, DTPA and DOTA (Figure 1.3.3).
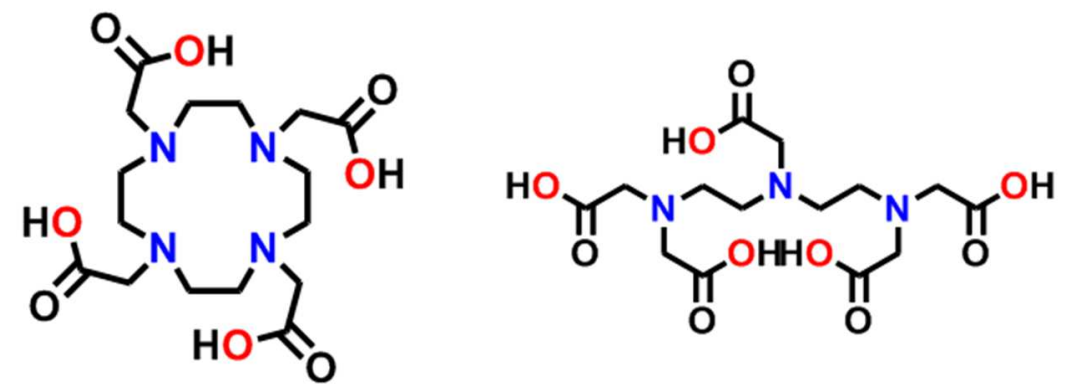

Figure 1.3.3. Parent ligand structures. DOTA (left) and DTPA (right) ligands. The coordinating atoms are shown in color for clarification. 
These two ligands both have nitrogen and oxygen donors that coordinate to lanthanide ions. Since lanthanide ions are hard acids, they prefer to interact with hard bases, such as oxygen and fluorine, over soft bases, like nitrogen, sulfur, and phosphorus. Nitrogen can be used as a donor atom, especially if there are oxygen donors present to help displace water which allows for the nitrogen donor to interact with the lanthanide ion. Due to the $4 \mathrm{f}$ electrons being inaccessible to incoming ligands and the lack of electrons in the $5 \mathrm{~d}$ shell, donor ligands as opposed to acceptor ligands are preferred by the lanthanide ions. Both DTPA and DOTA have amine and carboxylic acid donating ligands, which make them great ligands for the lanthanide metals.

DTPA and DOTA are also both octadentate ligands. This means that they coordinate to the lanthanide metal ion through 8 atoms. The coordination number of lanthanide complexes ranges from 8 to 10 depending on the ligands being used. Coordination numbers of lanthanide complexes are determined by the size of the metal ion and the size of the ligand rather than the number of ligands being used or the number of electrons in the complex. Coordination numbers of 8 and 9 are the most likely to occur in solution.
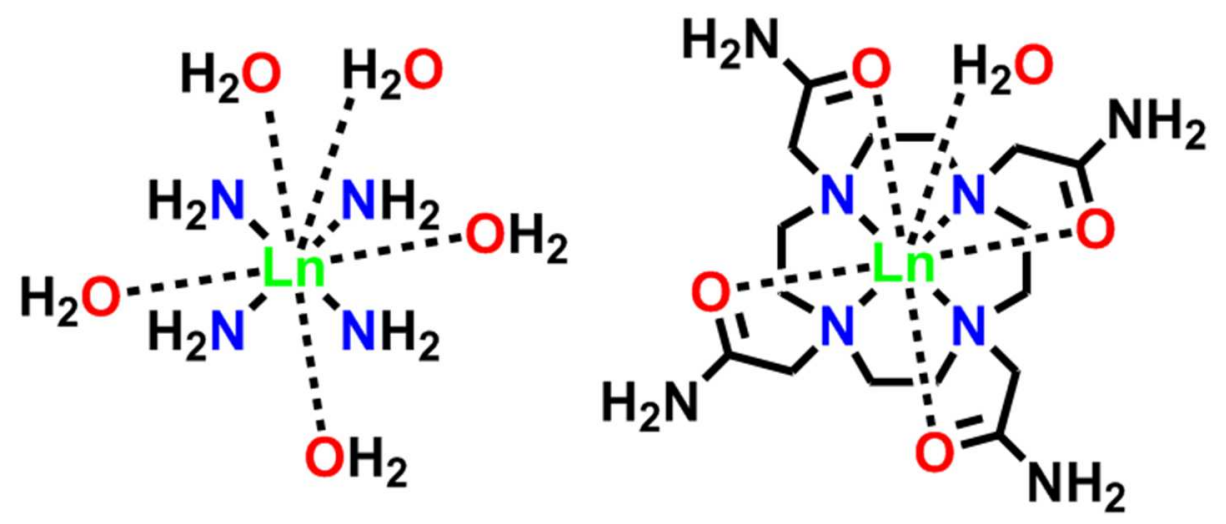

Figure 1.3.4. Ligand denticity. Monodentate molecules (left) and multidentate ligand (right). The coordinating atom is shown in color for clarification. 
Monodentate ligands (Figure 1.3.4), or ligands that coordinate to the metal ion through one site or atom, will afford coordination numbers of 8 or 9 and multidentate ligands (Figure 1.3.4), or ligands that coordinate to the metal ion through two or more sites or atoms, can afford a coordination number up to 10, and the larger the ionic radius of the lanthanide ion the higher the coordination number can be. It's difficult to achieve coordination numbers above 9 for monodentate ligands due to inter-ligand repulsions that may occur, but repulsions due to inter-donor atom interactions can be minimized by using donor atoms like oxygen or small molecule ligands with small bite angles.

Both DTPA and DOTA exhibit multidentate behavior. Multidentate ligands are more thermodynamically stable and have higher formation constants than monodentate ligands. Using a multidentate ligands increases the entropy of the system upon chelation because once one end of the ligand coordinates to the lanthanide ion, the concentration of the other end is artificially high and the equilibrium shifts so that the other end of the ligand also coordinates to the lanthanide ion (Figure 1.3.5).

\section{Increasing organization}

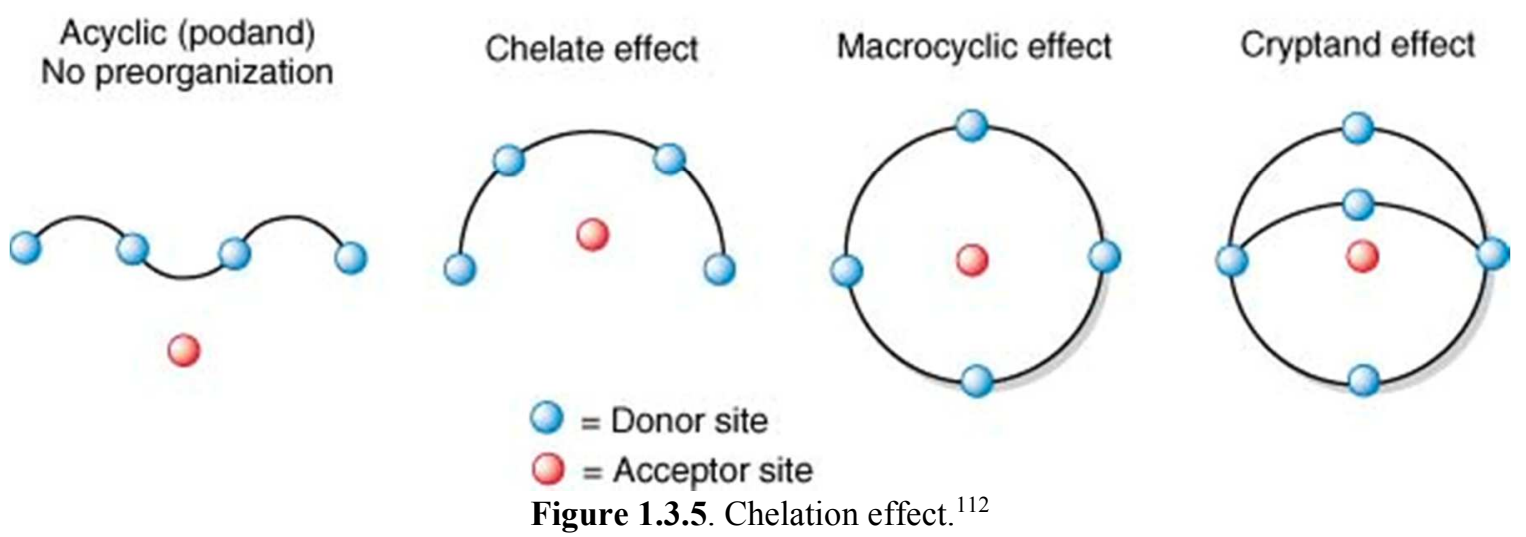


This process is especially amplified when using a macrocyclic system since the ligand more rigid and is unlikely to distort and move a ligating atom away from the coordination sphere. ${ }^{113}$ DTPA is a ligand with a linear backbone and is less thermodynamically stable than DOTA, which is a ligand with a macrocyclic ring backbone. It is advantageous to work with systems that are more thermodynamically stable when working with MRI contrast agents because $\mathrm{Ln}^{3+}$ metal ions are insoluble at physiological $\mathrm{pH}$ and temperature and are toxic when free in the body. ${ }^{111}$

\section{Chelate Isomerism}

When DOTA-like ligands coordinate lanthanide ions, there is a generally a coordination number of $9 ; 4$ co-planar oxygen donors above the lanthanide ion, 4 co-planar nitrogen donors below the lanthanide ion, and 1 water coordination site above the oxygen plane (Figure 1.3.6). ${ }^{114}$
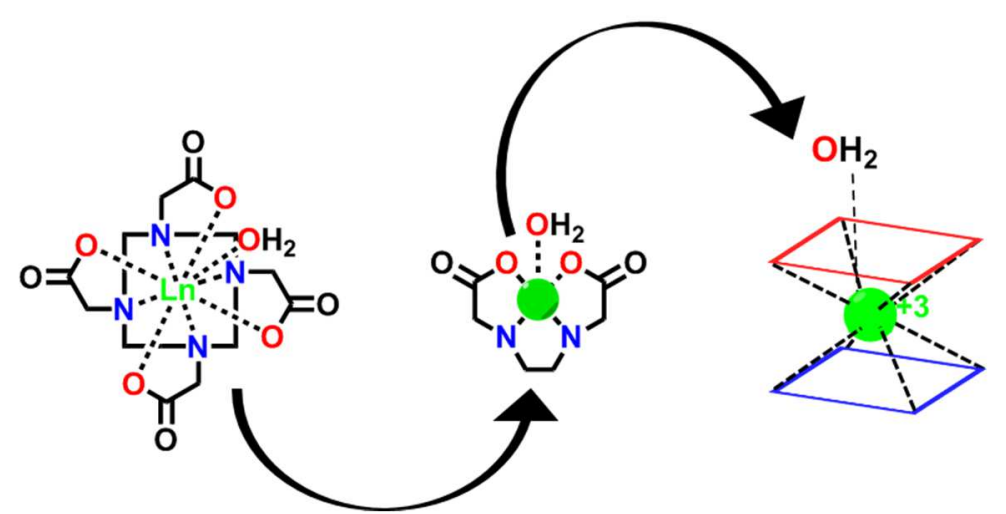

Figure 1.3.6. Visualizing the chelates. Left: A top-down view of the LnDOTA $^{-1}$. Center: A side view of the chelate structure, highlighting the nitrogen, oxygen planes surrounding the $\mathrm{Ln}^{+3}$ metal and the axially bound water molecule. Right: A visualization of the monocapped twisted square antiprism geometry DOTA-type chelates exhibit.

The water coordination site is important for the maximum relaxation of water inside the body and the rate at which the water molecule exchanges on and off of the chelate (water exchange rate) has an effect on the relaxivity of the contrast agent. The ring adopts 
the lowest energy conformation, which is the ideal 3333 ring conformation (Figure 1.3.7) for a macrocyclic ring. ${ }^{115}$

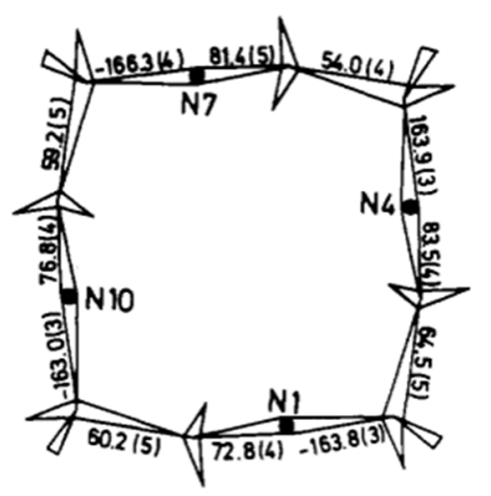

Figure 1.3.7. 3333 ring conformation. This conformation has 4 atoms ( 3 carbon and 1 nitrogen) and 3 bonds on each side of the square shape. ${ }^{116}$

The ethylene bridges of the macrocyclic ring have two different chiralities that can be adopted $(\delta$ or $\lambda)$ in this conformation, and the pendant arms also have two different chiralities that they can adopt $(\Delta$ or $\Lambda)$. All four macrocyclic bridges will adopt the same helicity (either $\delta \delta \delta \delta$ or $\lambda \lambda \lambda \lambda$ ) and the pendant arms will all adopt the same helicity (either $\Delta \Delta \Delta \Delta$ or $\Lambda \Lambda \Lambda \Lambda) \cdot{ }^{117,118}$ However, the ethylene bridges and the arms do not have to adopt the same helicity and therefore different combinations of bridge and arm helicity lead to four isomers available in solution (Figure 1.3.8, Figure 1.3.9)). The square antiprism (SAP) isomer occurs when the orientation of the arms and the ring are opposed $(\Lambda(\delta \delta \delta \delta)$ and $\Delta(\lambda \lambda \lambda \lambda))$ and the twisted square antiprism (TSAP) isomer occurs when the orientation of the arms and the ring agree $(\Delta(\delta \delta \delta \delta)$ and $\Lambda(\lambda \lambda \lambda \lambda)) .{ }^{119}$ 


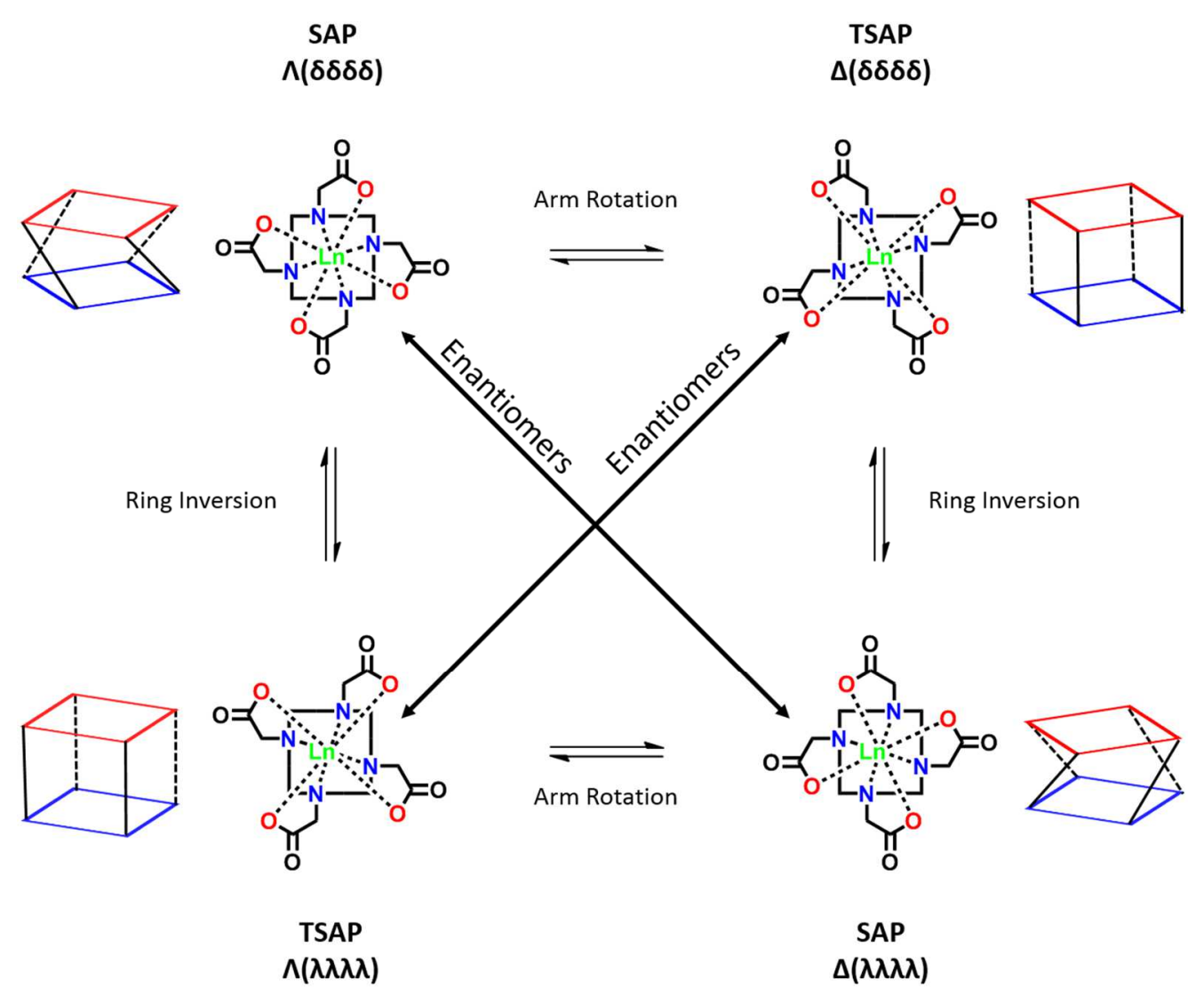

Figure 1.3.8. SAP and TSAP isomer interconversion.

SAP and TSAP coordination geometries are in dynamic exchange in solution and they differ only in the angle between the oxygen and nitrogen atoms when looking down upon the chelate; SAP coordination geometries have a torsion angle close to $40^{\circ}$ and TSAP coordination geometries have a torsion angle close to $25^{\circ}$. The four isomers are in constant interconversion, and the interconversion between the two coordination geometries is possible through arm rotations and macrocyclic ring inversions (Figure 1.3.8, Figure 1.3.9). ${ }^{120-122}$ 


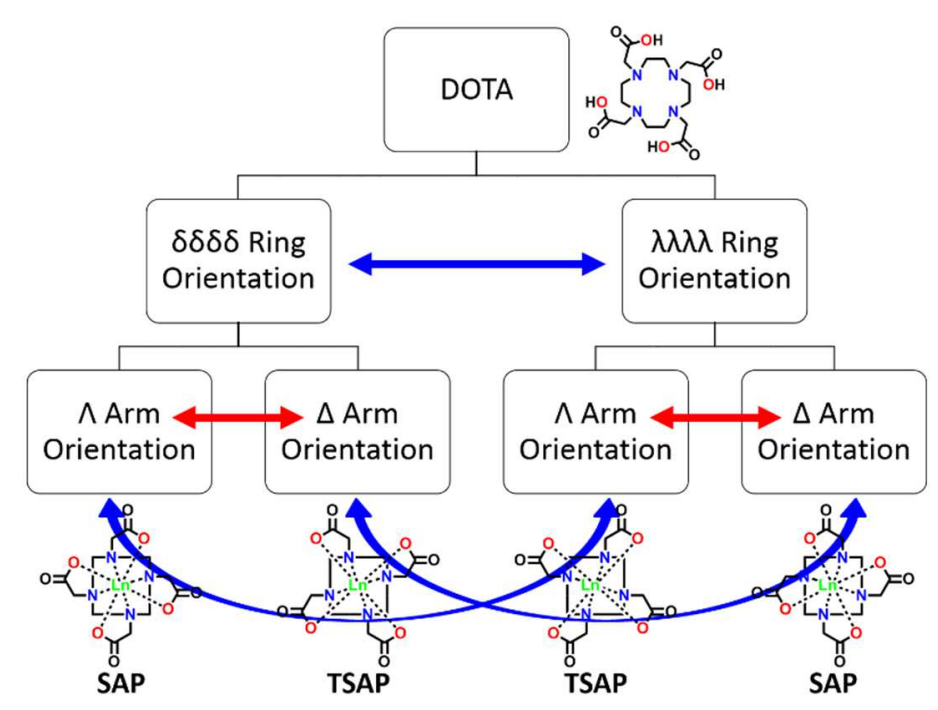

Figure 1.3.9. Unsubstituted isomer flowchart. The red arrows indicate interconversion between SAP and TSAP isomers through arm rotations and the blue arrows indicate interconversion through ring inversions. The bottom blue arrows are present to show which isomers are converting when the ring inversions occur.

The two coordination geometries also have different chemical properties, with the TSAP isomer having a water proton exchange rate 50 to 100 times faster than the SAP isomer due to the elongated cage in the TSAP isomer. ${ }^{102}$ This means that a solution of any given contrast agent utilizing the DOTA ligand is a mixture of isomers. MRI scans with contrast require a large dose of contrast agent (in grams) in order to achieve useful images and decreasing the dose would be beneficial. Isolating one more effective geometry over the other less effective geometry could lead to using a lower dose of contrast agent per scan that is more effective than the mixture of isomers.

The isolation of isomers is possible by utilizing substitutions on the contrast agent cage and halting the interconversion processes. Upon substitution on the macrocyclic ring, ring inversions are halted but arm rotations are still available for interconversion (Figure 1.3.10). ${ }^{123}$ If substitutions are made on the arms, arm rotations are halted but ring inversions are still available for interconversion (Figure 1.3.11). ${ }^{124}$ When substitution on the 
macrocyclic ring is used, the substituent is spatially placed in the equatorial position and the ring ethylene bridges adopt only the $\delta \delta \delta \delta$ helicity.

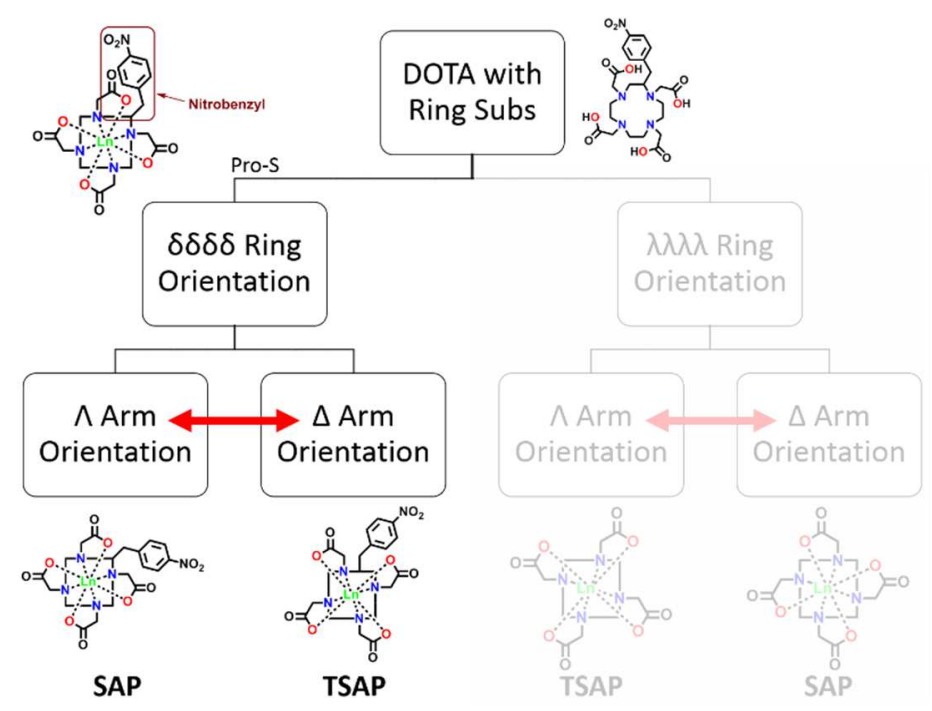

Figure 1.3.10. Ring substitution isomer flow chart. This substitution completely locks out the $\lambda \lambda \lambda \lambda$ ring orientation.

When substitution on the pendant arms is used, isomers formed are dependent on the stereochemical configuration at the $\alpha$ position and whether the arm orientation matches the orientation of the ring (Figure 1.3.11).

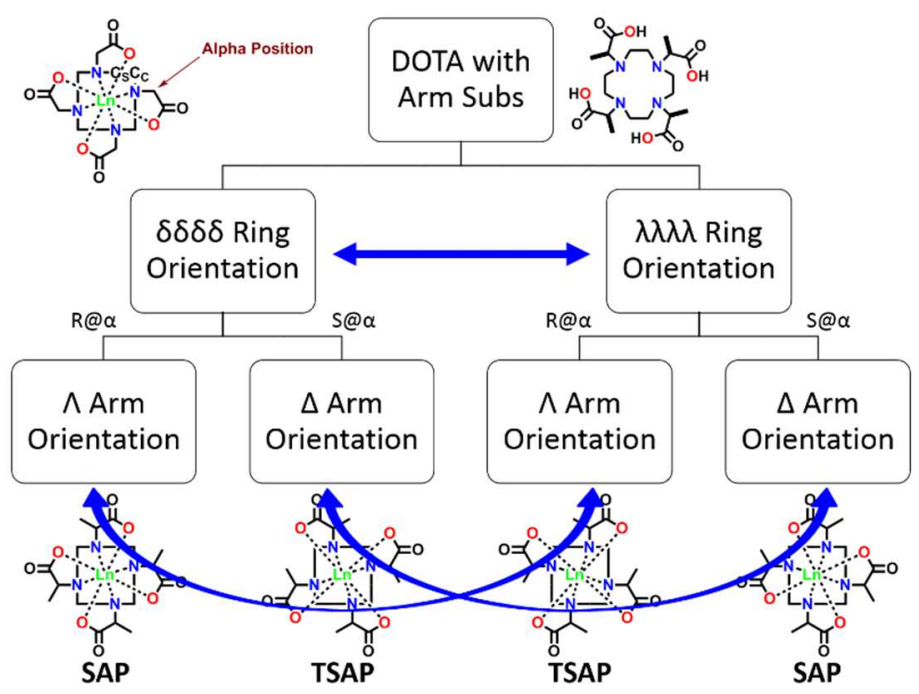

Figure 1.3.11. $\alpha$ position arm substitution isomer flowchart. 
When both substitutions are utilized together, both the arm rotations and ring inversions are halted, creating isomers which can be separated from one another using HPLC. ${ }^{125-128}$ It was expected that only two isomers would be present when both ring and arm substitutions were utilized, since substitution on the ring allows for only the $\Lambda(\delta \delta \delta \delta)$ and $\Delta(\delta \delta \delta \delta)$ isomers to be present. However, it was found that there were actually four isomers. Two SAP isomers and two TSAP isomers were present in solution, leading to the discovery that when substitutions are put on the macrocyclic ring, regioisomers develop (Figure 1.3.12). On the macrocyclic ring, with the 3333 ideal ring conformation, there are side and corner carbons. ${ }^{126}$

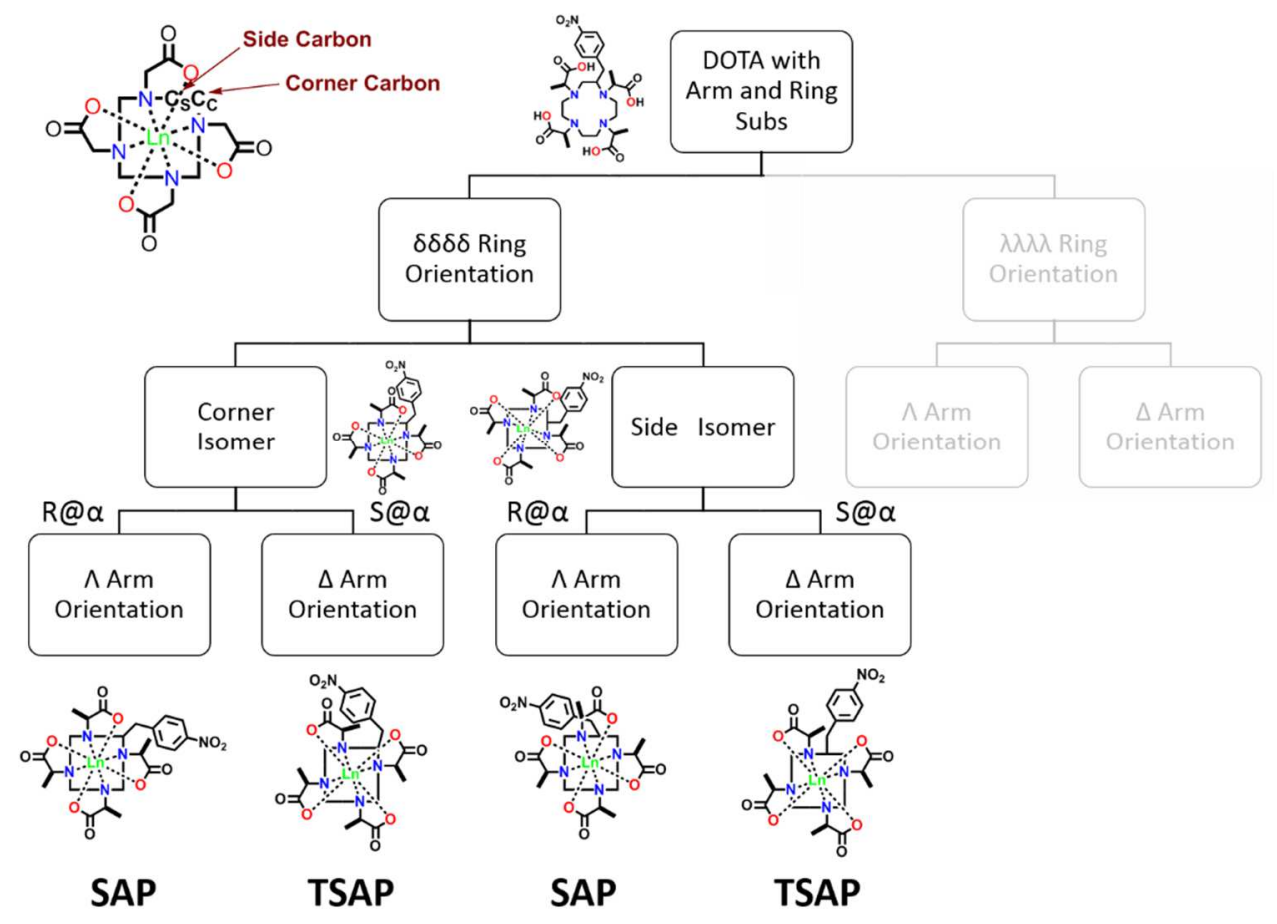

Figure 1.3.12. Regioisomers flow chart. Utilizing arm and ring substitutions led to the discovery of regioisomers.

The substituent on the macrocyclic ring will exist in the equatorial position on the side or corner carbon, and the two regioisomers are not interconvertible unless the metal is 
released from the ligand and re-chelated. Separation of the regioisomers is therefore possible utilizing HPLC.

In order to ease the synthesis of substituted chelates, the idea of $\delta$ substituted arms controlling arm helicity was suggested. Moving the stereocenter away from the alkylated carbon (in the $\alpha$ position) would make synthesis easier and it was unknown if the $\delta$ position would control helicity in the same way that the $\alpha$ position did. Substitution at the $\delta$ position of the arm does not halt arm rotations but may play an important role in where the arm substituent resides in space. ${ }^{129}$ The substituent's position in space starts in a pseudoequatorial position, with the arm helicity conforming (Figure 1.3.13).

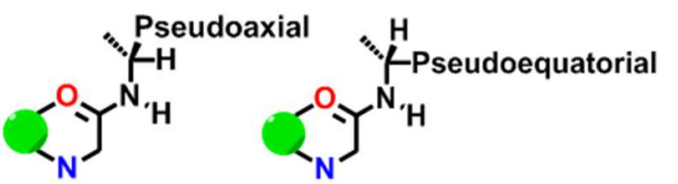

Figure 1.3.13. Delta substitution effects. The substituent's placement in space affects the water exchange kinetics of the chelate.

Arm rotations still occur with $\delta$ position substitutions and the arm may rotate to a different helicity, which then moves the substituent to a pseudo-axial position (Figure 1.3.13). If the substituent is a hydrophobic moiety and located in the pseudo-axial position, closest to the water exchanging site, water exchanging on and off of the chelate is slowed since the water has to get past several hydrophobic substituents. If the hydrophobic substituent is located in the pseudo-equatorial position, the exchanging water is farther away from the hydrophobic moieties and can exchange without interference. The opposite is true if the substituent is hydrophilic. Hydrophilic substituents in the pseudo-axial position increase the water exchange rate of the chelate because the moieties increase the amount of water molecules in the pool of water near the exchanging site, and hydrophilic 
substituents in the pseudo-equatorial position decrease the number of waters in the pool of water near the exchanging site by moving them farther away from the site and therefore decreases the water exchange rate of the chelate. The first part of this work looks specifically at what happens in these DOTAM systems when the substituent is hydrophobic instead of hydrophilic.

\subsection{Progressing the MRI Technique}

\section{Theory}

The medical imaging field is constantly developing and improving, and the MRI technique is extremely versatile due to that constant development. Along with the typical $\mathrm{T}_{1^{-}}, \mathrm{T}_{2^{-}}$, and PD-weighted images that are generally taken in MRI exams, special exams, specialized contrast agents, and a wide range of pulse sequences make the technique quite powerful.

Diffusion MRI exams diagnose stroke and multiple sclerosis by measuring the diffusion of water in various tissues, MR angiography (MRA) and MR venography (MRV) (Figure 1.4.1) exams evaluate the arteries, veins, and blood flow of the patient and whether or not stenosis or aneurysms are occurring ${ }^{130-133}$, functional MRI (fMRI) exams (Figure 1.4.1) claim to detect increased blood flow and as a result show which part of the brain is being stimulated and used during different tasks or during emotions ${ }^{31,52,134,135}$, and MR spectroscopy (MRS) exams (Figure 1.4.1) can detect changes in biomarkers in tissues and can help to diagnose gliomas, non-glial tumors, infection, ischemia and infarctions, mitochondrial disorders like Leigh syndrome, hepatic encephalopathy, and white matter diseases like progressive multifocal leukoencephalopathy (PML) and Canavan 
disease. ${ }^{78,136-140}$ Each exam type utilizes different pulse sequences to look specifically at a wanted signal and to disregard the other signals present. Real-time MRI has been a development goal for several decades and is often called FLASH MRI (fast low angle shot magnetic resonance imaging). ${ }^{141}$ This type of exam has been able to shorten the length of exam times, yields 3-dimensional representations of vasculature and complex anatomic structures like the brain and joints, and has been used to create movies of beating hearts and to guide minimally invasive surgical procedures in interventional MRI.
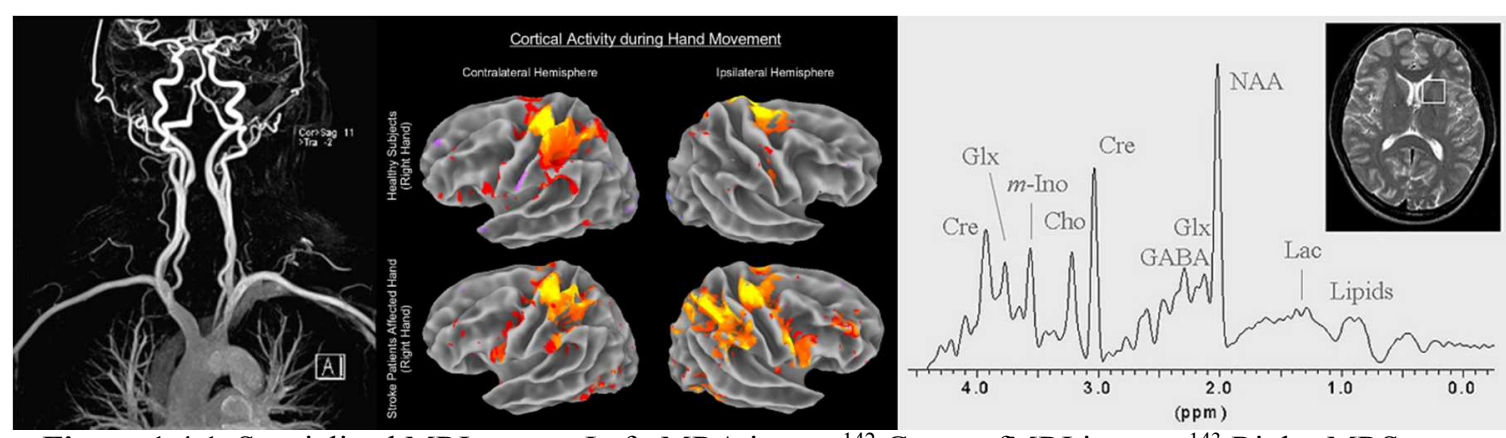

Figure 1.4.1. Specialized MRI exams. Left: MRA image. ${ }^{142}$ Center: fMRI images. ${ }^{143}$ Right: MRS exam results. ${ }^{144}$

Specialized contrast agents can be used to enhance contrast or to target the exam to a specific part of the body. Specialized MRI contrast agents include responsive agents ${ }^{86,145-}$ ${ }^{150}$, active targeting agents ${ }^{151-155}$, organ specific agents ${ }^{156-158}$, and $\mathrm{pH}$-sensitive agents ${ }^{148,159-}$ 165. These various agents can turn on or off if enzymatically cleaved, can indicate if certain metabolites or metals are present in the body, can target organs, tumors, cells, proteins, and antibodies, and can indicate $\mathrm{pH}$, temperature, or oxygen levels in the body. The combined power of specialized exams and targeted agents means that MRI as a diagnostic tool can be extremely powerful. With the advancement of the exams and the contrast agents used, the analyses of the data are increasingly difficult and require quite a bit of manipulation 
and fitting of the signal data to mathematical expressions and comparing to collected laboratory data.

Currently, parameters that are used for fitting the data collected to make images and maps vary and there is no one method. In fact, there are many different methodologies for calculating information from data collected and there are many assumptions that go into fitting the data. For example, when utilizing CEST imaging to determine the $\mathrm{pH}$ of a sample, the following are the parameters needed for one type of calculation: the RF labelling coefficient, the spillover factor, the chemical exchange rate from labile protons to bulk water protons, the ratio of labile protons to the bulk water protons, and the intrinsic longitudinal relaxation rate of bulk water. The labelling coefficient and spillover factor are calculated from the power levels and offset of the RF irradiation, the labile proton frequency, the exchange rate, and the apparent longitudinal and transverse relaxation times. Determining all of the parameters is often difficult or not possible and assumptions for some of the parameters have to be made to make the calculation work so relevant information can be extracted from the image. The above is only one method for calculating pH utilizing a CEST agent, and there are many different calculations and approaches that researchers have come up with. ${ }^{148,159,160,164,166-177}$ Each method has its own list of parameters, some being held and some being allowed to fluctuate in calculations. Determining which parameters to hold constant and which to allow to be fluid, and having that protocol be universal, doesn't seem to be as big of a goal as it should be.

Compartmentalization is another problem that should be addressed with the advancement of the MRI technique. Calibration curves and information used in the 
determination of relevant information, like $\mathrm{pH}$ and temperature, are often generated in a lab setting inside test tubes, beakers, phantoms, and NMR tubes. These environments are free of compartmentalization, where the agents in question have free access to water and other molecules present in testing solutions. In vivo environments are very limited environments with separated compartments as opposed to a homogenous solution. The comparison from one to the other is a gross error. Exchange rates where the chelate has unlimited access to water will give you accurate information for that system, and that system only. Trying to apply the open system's information to a different system where the chelate has limited access to water will not give you accurate results, which leads to inaccurate $\mathrm{pH}$ and temperature maps and erroneous values.

Compartmentalization in the laboratory setting should allow for a controlled encapsulated environment where chelates have access to a limited amount of water. Analyzing chelates in this system should lead to more accurate values being produced from data collected. Understanding the full mechanism of exchange and how that process affects the information gained from collected data is vital. The second part of this work looks at the encapsulation of contrast media inside phospholipid vesicles and understanding the effects of water exchange across the membrane.

\section{Internal CEST}

While the second part of this work could have a global application on the MRI field, it also has a more focused goal. Encapsulating the contrast agent inside a phospholipid bilayer has the potential to slow and control water exchange kinetics depending on the lipids chosen for the vesicles. This method for controlling water exchange rates in chelates 
involves looking at affecting the environment that the chelate sees instead of changing the ligand of the chelate. By encapsulating a paraCEST chelate within a semipermeable membrane, like a phospholipid bilayer, the chelate would only "see" the water that is inside the vesicle. ${ }^{178}$ Regardless of whether the water exchange rate of the chelate, $\mathrm{k}_{\mathrm{c}}$, is fast or slow, the observed water exchange rate, $\mathrm{k}_{\mathrm{d}}$, will be slow due to the slow diffusion of water across the phospholipid bilayer membrane (Figure 1.4.2). This slow exchange would allow relaxed water protons to move out into the bulk water at such a slow rate that the observed water exchange rate would be significantly slower than what would be expected with just the chelate and allows for improved contrast. Here, the water exchange rate of the chelate isn't actually changed, but the observed water exchange rate is. This methodology allows for a more facile manipulation of the water exchange rates. The steps of encapsulating chelates inside phospholipid bilayers take significantly less time and eliminate lengthy and difficult organic syntheses. However, there are variables that should be considered in the design of the encapsulation system.

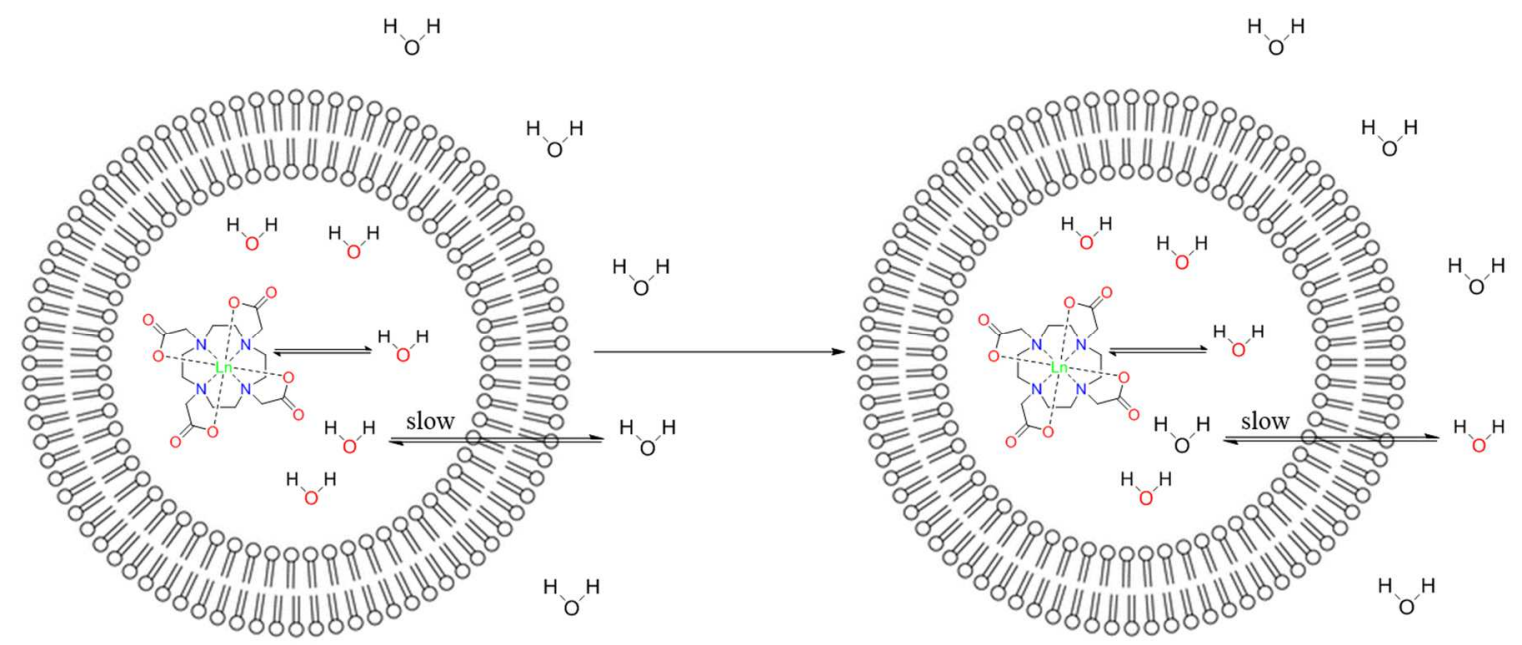

Figure 1.4.2. Internal CEST mechanism. 


\subsection{Vesicles 101}

\section{Lipid Structure and Function}

Lipid composition is picked based on desired characteristics of the system. Generally the lipids have a hydrophilic phosphate head and a hydrophobic tail that contains two fatty acid chains (Figure 1.5.1).

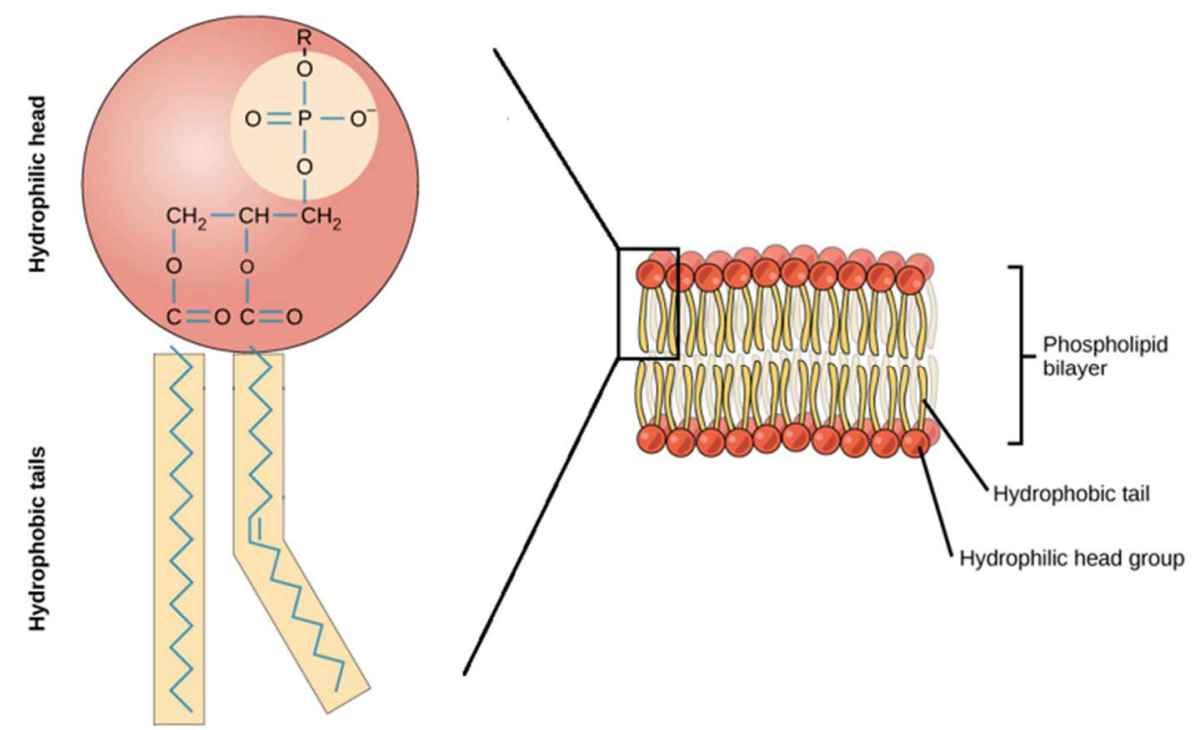

Figure 1.5.1. Lipid structure and phospholipid bilayer. ${ }^{179}$

The head groups have the ability to change the surface chemistry of the vesicle, can serve as a site for anchoring targeting molecules, or can also be signals for other molecules. Sphingolipids are an example of a particular polar head group that provides a charged group on the surface of the liposome. Phosphatidylserine (PS) is a signal for cell apoptosis in mammalian red blood cells ${ }^{180}$, but when it is incorporated into growth plate vesicles it aids in bone mineralization ${ }^{181}$. The head groups determine how the vesicle interacts, or doesn't, with its surrounding environment. ${ }^{182,183}$

The tails of the lipid have the ability to affect the liposome's membrane properties based on the length of the tail, the chemical properties of the tail, and whether or not the 
chains of the tail are saturated or unsaturated. Whether or not the lipid tail is saturated or unsaturated will affect the packing of the tail inside the bilayer and affects the liposomes resistance to stressors, like stretching or bending. ${ }^{184-188}$ The length of the chain will also affect the packing ability of the lipid tails, with longer tails requiring more bending and folding to fit inside the bilayer. ${ }^{184-188}$ The chemical properties of the tail will determine the transition temperature for the bilayer (Table 1.5.1). Below the transition temperature, the bilayer adopts a solid gel phase, while above the transition temperature the bilayer will enter into a fluid state. ${ }^{189-202}$ The solid gel phase makes the liposomes more stable and better able to handle stresses by locking in the lipids and limiting their mobility, which also in turn makes the exchange rate across the membrane slower. Being above the transition temperature during formation allows lipids to diffuse and move within the membrane ${ }^{203}$, which allows the vesicle to be manipulated into the desired size and lamellarity. Tail length can affect the transition temperature, with longer chains having more surface area available to interact with other tails which decreases the lipid mobility and has a higher transition temperature. Saturation also affects the transition temperature, with unsaturated lipids have disrupted packing within the layer which creates more mobility, and in turn decreases the transition temperature. 
Table 1.5.1. Phospholipid transition temperature. ${ }^{204}$

\begin{tabular}{lll} 
Phospholipids & Abbreviation & \multicolumn{1}{c}{$\mathrm{T}_{c}(\mathrm{C})$} \\
\hline $\begin{array}{l}\text { Soybean phosphatidylcholine } \\
\text { Hydrogenated soybean }\end{array}$ & SPC & -20 to -30 \\
$\quad$ phosphatidylcholine & HSPC & 52 \\
Egg sphingomyelin & ESM & Ca. 40 \\
Egg phosphatidylcholine & EPC & -5 to -15 \\
Dimyristoyl phosphatidylcholine & DMPC & 23 \\
Dipalmitoyl phosphatidylcholine & DPPC & 41 \\
Dioleoyl phosphatidylcholine & DOPC & -22 \\
Distearoyl phosphatidylcholine & DSPC & 55 \\
Dimyristoyl phosphatidylglycerol & DMPG & 23 \\
Dipalmitoyl phosphatidylglycerol & DPPG & 41 \\
Dioleoyl phosphatidylglycerol & DOPG & -18 \\
Distearoyl phosphatidylglycerol & DSPG & 55 \\
Dimyristoyl & DMPE & 50 \\
phosphatidylethanolamine & & \\
Dipalmitoyl & DPPE & 60 \\
phosphatidylethanolamine & & \\
Dioleoyl & DOPE & -16 \\
phosphatidylethanolamine & & \\
Dimyristoyl phosphatidylserine & DMPS & 38 \\
Dipalmitoyl phosphatidylserine & DPPS & 51 \\
Dioleoyl phosphatidylserine & DOPS & -10
\end{tabular}

Phospholipids are used as the primary composition of vesicles. Naturally occurring phospholipids (Figure 1.5.2) include phosphatidylcholine (PC), phosphatidylethanolamine (PE), phosphatidylserine (PS), phosphatidylinositol (PI), and phosphatidylglycerol (PG). These are general lipid names and are either saturated or unsaturated. 


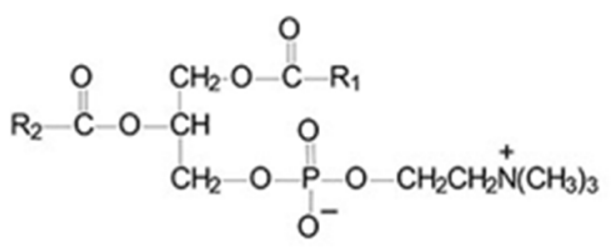

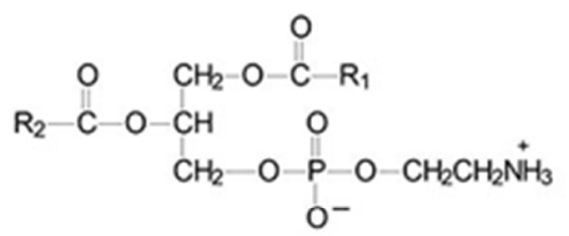

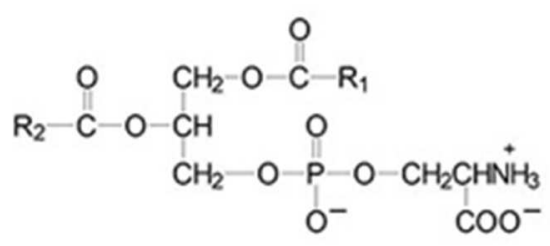

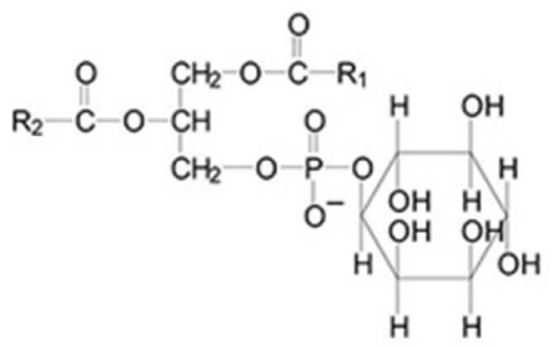<smiles>[R]C(=O)OCC(COP(=O)([O-])OCC(O)CO)OC([R])=O</smiles>

Phosphatidylcholine (PC)

Phosphatidylethanolamine (PE)

Phosphatidylserine (PS)

Phosphatidylinositol (PI)

Phosphatidylglycerol (PG)

Figure 1.5.2. Naturally occurring phospholipid head groups.

Commonly used saturated lipids (Figure 1.5.3) are generally dipalmitoyl derivatives of the aforementioned phospholipids: dipalmitoyl phosphatidylcholine (DPPC), distearoyl phosphatidylcholine (DSPC), dipalmitoyl phosphatidylethanolamine (DPPE), dipalmitoyl phosphatidylserine (DPPS), dipalmitoyl phosphatidic acid (DPPA), and dipalmitoyl phosphatidylglycerol (DPPG). 


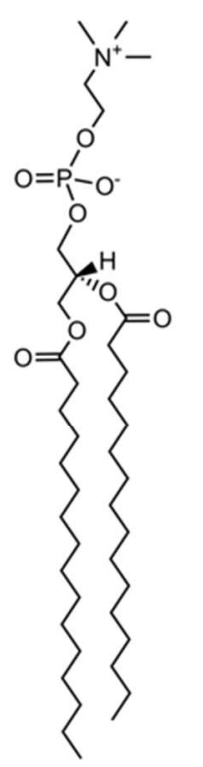

DPPC

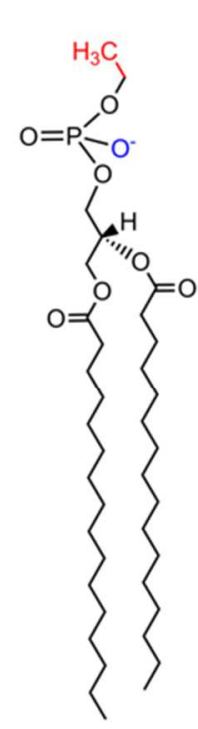

DPPeth

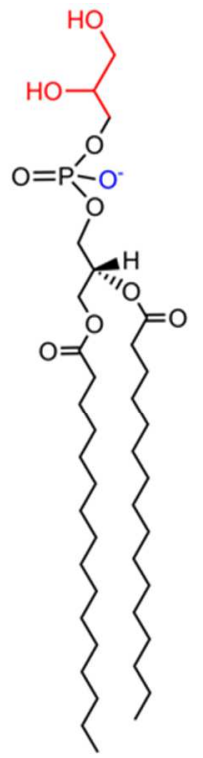

DPPG
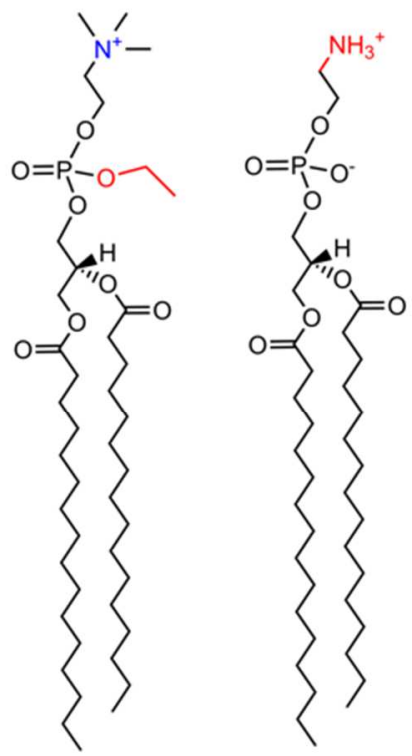

EDPPC

DPPE

Figure 1.5.3. Saturated lipids. Examples of saturated lipids.

Commonly used unsaturated lipids (Figure 1.5.4) are dioleoyl derivatives of the aforementioned phospholipids: dioleoyl phosphatidylcholine (DOPC) and dioleoyl phosphatidylglycerol (DOPG).

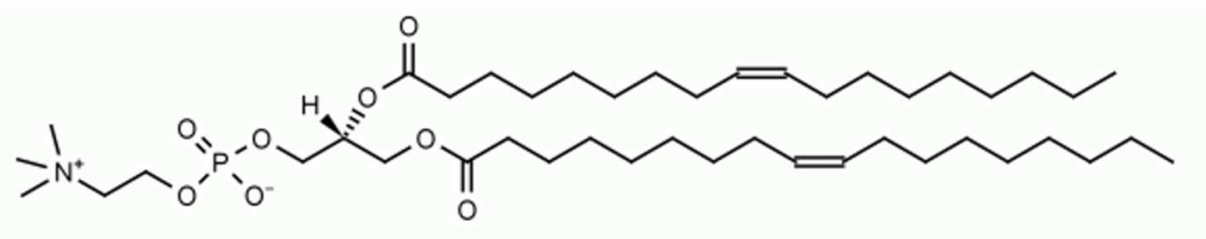

DOPC<smiles>CCCCCCCC/C=C\CCCCCCCCC(=O)OC[C@H](COP(=O)([O-])OCC(O)CO)OC(=O)CCCCCCC/C=C\CCCCCCCC</smiles>

\section{DOPG}

Figure 1.5.4. Unsaturated lipids.

To increase the bilayer stability and decrease the fluidity of the membrane, sterols, like cholesterol, can be incorporated into the liposome bilayer. Cholesterol (C) (Figure 1.5.5) intercalates into the bilayer between the tails of the phospholipids and reduces the permeability of the membrane to water soluble molecules. ${ }^{190,205-209}$ 


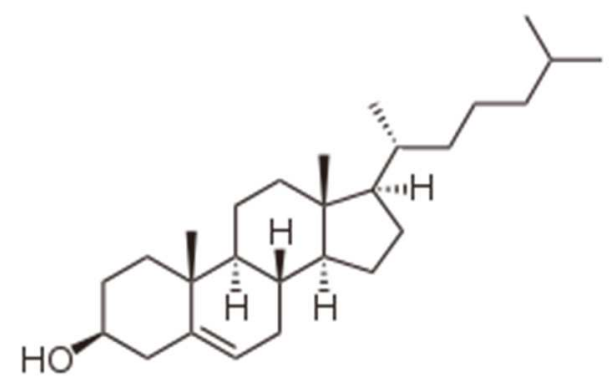

Figure 1.5.5. Cholesterol structure.

\section{Vesicle Classification and Preparation}

Phospholipid vesicles can be classified in three ways: by structural parameters, by method of preparation, or by composition and application. The structural features that classify a vesicle are the lamellarity and the size of the vesicle. The lamellarity of a vesicle is determined by the number of bilayers that make up the vesicle and the number of aqueous compartments. If the vesicle is made up of one bilayer that surrounds one aqueous compartment, then the vesicle is considered to be a unilamellar vesicle (ULV). If the vesicle has one internal compartment but is made up of 2 or more bilayers stacked on top of each other, then the vesicle is considered to be an oligolamellar vesicle (OLV). If the vesicle has several bilayers and has several compartments on the inside of the vesicle, then the vesicle is considered to be a multilamellar vesicle (MLV) (Figure 1.5.6). The size of vesicles ranges from $10 \mathrm{~nm}$ to several $\mu \mathrm{m}$. Vesicles that are generally between 20 and $40 \mathrm{~nm}$ are considered small vesicles, 40 to $80 \mathrm{~nm}$ size vesicles are considered medium vesicles, and vesicles between $100 \mathrm{~nm}$ and $1 \mu \mathrm{m}$ are considered large vesicles. Generally, ULVs can be small (SUV), medium (MUV), and large (LUV) (Figure 1.5.6), while MLVs are usually LUVs or OLVs enclosing a large number of SUVs. 

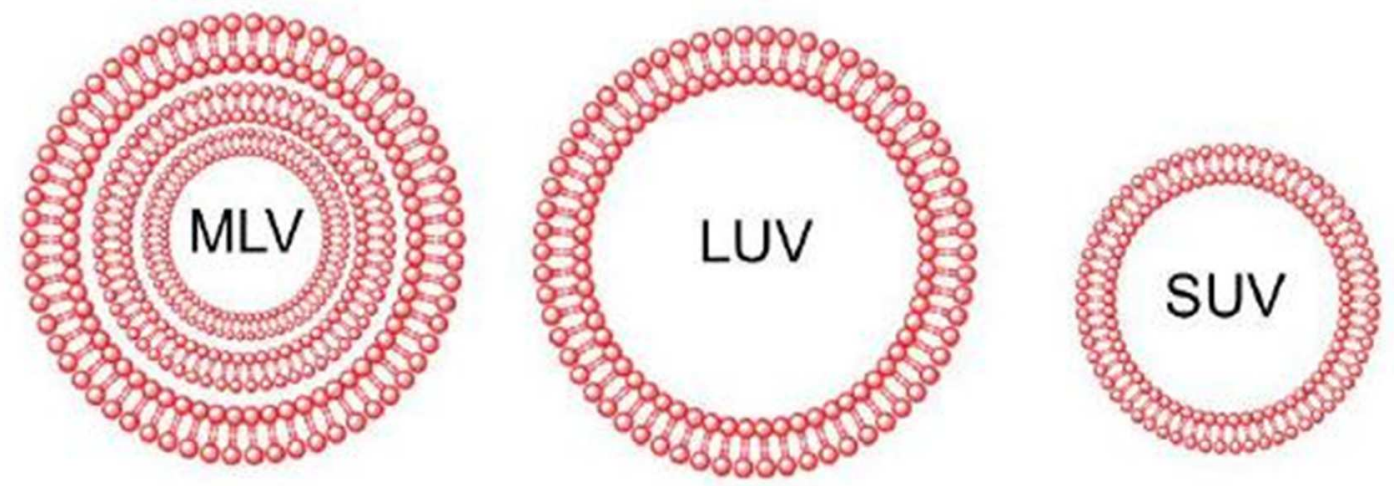

Figure 1.5.6. Vesicle classification. ${ }^{210}$

Vesicles can be classified by the method of preparation, which can influence the size and lamellarity of the vesicles. Each method below begins by dissolving the lipid mixture in chloroform, or some other similar solvent, and removing the solvent under reduced pressure so that the lipid is dried into a thin layer on the glassware (typically a round bottom flask). After that, the method of formation of vesicles differs and generally ends with an extrusion process to control the size of the sample. Generally though, when the thin films are hydrated with aqueous medium, MLVs form with 5-10\% of the aqueous volumes enclosed within the lipid membranes. Lipids have polar, hydrophilic head groups and nonpolar, hydrophobic fatty acyl chains. The vesicles form spontaneously due to the hydrophobic effect, where the hydrophobic tails folding against one another increases the entropy of the system, causing more tails to continue interacting while the hydrophilic head groups are placed on the outer areas of the bilayer in contact with the aqueous medium (Figure 1.5.7). Other working forces in the formation of the vesicles include van der Waals forces, electrostatic forces, and hydrogen bonds. 


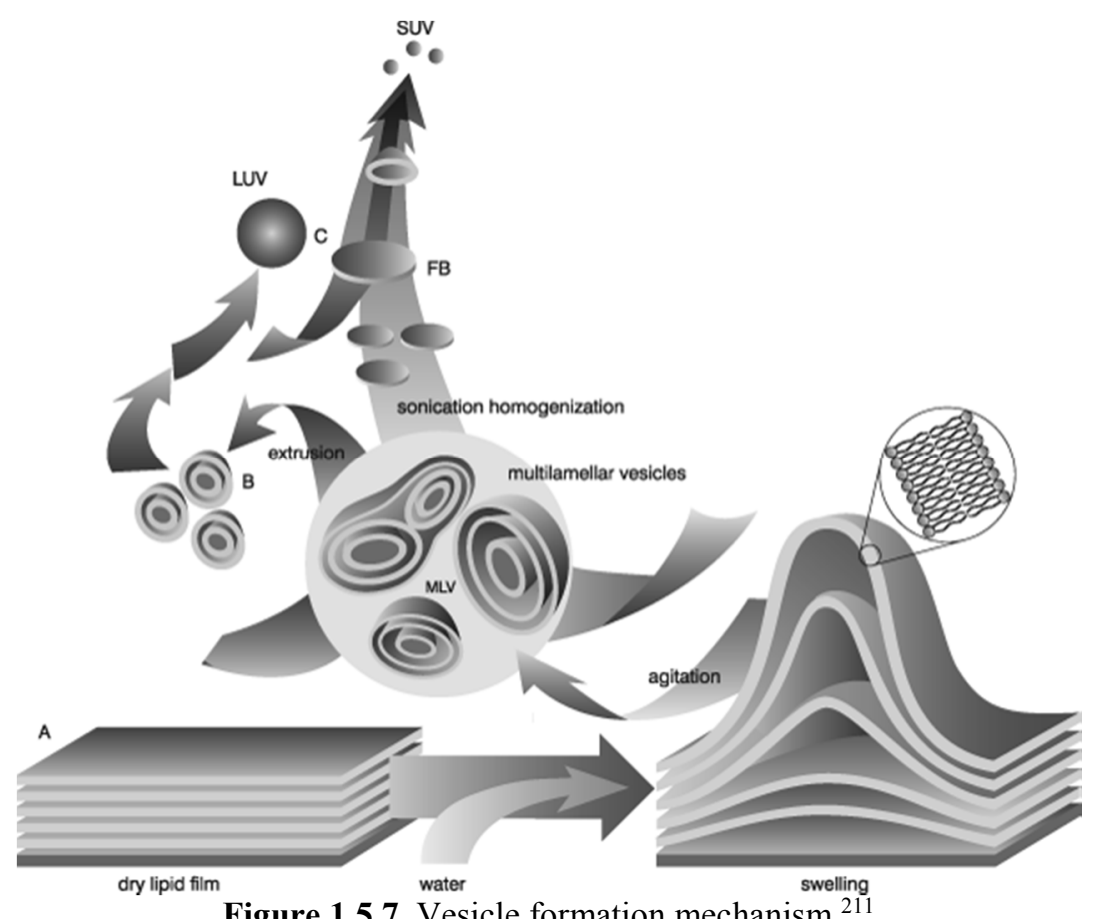

Forming vesicles can be done in a number of ways, however, forming the vesicles and having some control over the size of the vesicles is typically done with either extrusion or sonication. ${ }^{212}$ Sonicating at certain frequencies will influence what size the vesicles generally are, with faster frequencies making smaller vesicles and slower frequencies resulting in larger vesicles. Extrusion is a much more precise method for controlling the size of the vesicles. The extrusion apparatus used can either be manual (two syringes on either side of porous filters, operated with your hands) or it can be a pressurized system that uses inert gas to force the sample through the porous filters. The porous filters are polycarbonate membranes that are whatever size is desired for the vesicles being made. Sonication and extrusion are used to make homogenous samples and can be used in combination with each other, with other methodologies, or on their own. Both techniques can make ULVs with tunable small size distributions. 
The reverse-phase evaporation method ${ }^{212}$ involves suspending the desired lipid mixture in an organic solvent, like diethyl ether or isopropyl ether, so that the reverse phase vesicles are formed with the polar heads on the inside of the bilayer and the nonpolar tails on the outside of the bilayer. The aqueous phase is added to the mixture, forming a two-phase system which is sonicated briefly until the mixture becomes either a clear one-phase dispersion or a homogenous opalescent dispersion. The organic solvent is then removed under reduced pressure, leaving only the aqueous mixture with formed vesicles. The vesicles can then be extruded to the desired size. If the vesicles formed are SUVs or OLVs, the vesicles are called REV vesicles. If the vesicles formed are MLVs, the vesicles are called MLV-REV vesicles.

The freeze-thawed preparation ${ }^{212}$ involves hydrating the thin layer with the aqueous solution or buffer and vortexing the sample to emulsify the lipid mixture. The container that the sample is in is then dipped in liquid nitrogen for some time and then placed in a hot water bath at some temperature for thawing for some time. The time frames picked can differ based on the paper, but generally the samples are frozen and heated for the shortest amount of time, and the temperature of the hot water bath should be above the transition temperature of the lipid mixture. The freeze-thaw cycle is repeated for a designated number of rounds, usually 10 . After this process is completed, the samples are either extruded or sonicated to further form the vesicles and control the size and lamellarity of the vesicles. However, the process of freeze-thawing seems to form predominately unilamellar vesicles, and vesicles made using this technique are called FAT-ULVs. 
The dehydration-rehydration process ${ }^{212}$ involves hydrating the thin film of lipid with the aqueous solution or buffer, and then extruding the sample to the desired size. After the extrusion process, the formed liposomes are dehydrated by freeze-drying for some time until the sample is dry, which typically is $24-26$ hours. The dehydrated liposomes are sealed and stored at $4^{\circ} \mathrm{C}$, if desired, then brought back to room temperature and slowly hydrated again with the buffer being used, with small increments of buffer being added over an hour at a temperature above the transition temperature of the lipid mixture. This method also produces predominately unilamellar vesicles, and the formed vesicles are called DRVs.

The third classification category is composition and application. This depends on the charge of the lipid, but certain lipid combinations have been identified as excellent targeting systems and are then classified by the use of the vesicle. If the lipids being used contain neutral or negatively charged phospholipids, with or without added cholesterol, the vesicles are called conventional liposomes (CL), and if the lipid mixture contains cationic lipids then the vesicles are called cationic liposomes. ${ }^{213}$ Fusogenic liposomes (RSVE) are made from reconstituted Sendai virus envelopes, which makes them good drug delivery systems that deliver to the cytoplasm in an endocytosis-independent way. ${ }^{214-217}$ Long circulatory liposomes (LCL), or stealth liposomes, are liposomes that have PEG derivatives attached to the surface of the liposome. ${ }^{218-221}$ The PEG derivatives decrease the detection by phagocyte systems, which allows the liposomes to have an extended circulation time in the body. Immune-liposomes are either CLs or LCLs that are targeted with monoclonal antibodies or other recognition sequences on the surface of the liposome. ${ }^{222-225} \mathrm{pH}$ sensitive liposomes are also possible with the right mix of lipids. These vesicles are systems that fall 
apart when they encounter a certain $\mathrm{pH}$ in the body. ${ }^{226-231}$ These systems are often used in combination to get the best system for the desired application.

\section{Vesicle Characterization}

Vesicle shape can be visualized using electron microscopic techniques, while lamellarity of the vesicle can be evaluated using freeze-fracture electron microscopy and ${ }^{31} \mathrm{P}$ NMR. The size and size distribution of the sample can be determined using many any or all of the following methodologies: light microscopy, fluorescent microscopy, electron microscopy (particularly TEM), laser light scattering photon correlation spectroscopy, field flow fractionation, gel permeation, and gel exclusion. Laser light scattering methods, like dynamic light scattering (DLS), is the most simple and rapid methodology and measures an average property of the bulk liposomes. TEM and other electron microscopy techniques are the most precise method for size determination because it allows the visualization and measurement of individual liposomes (Figure 1.5.8); however, electron microscopy techniques are often time consuming and the equipment is not always available.

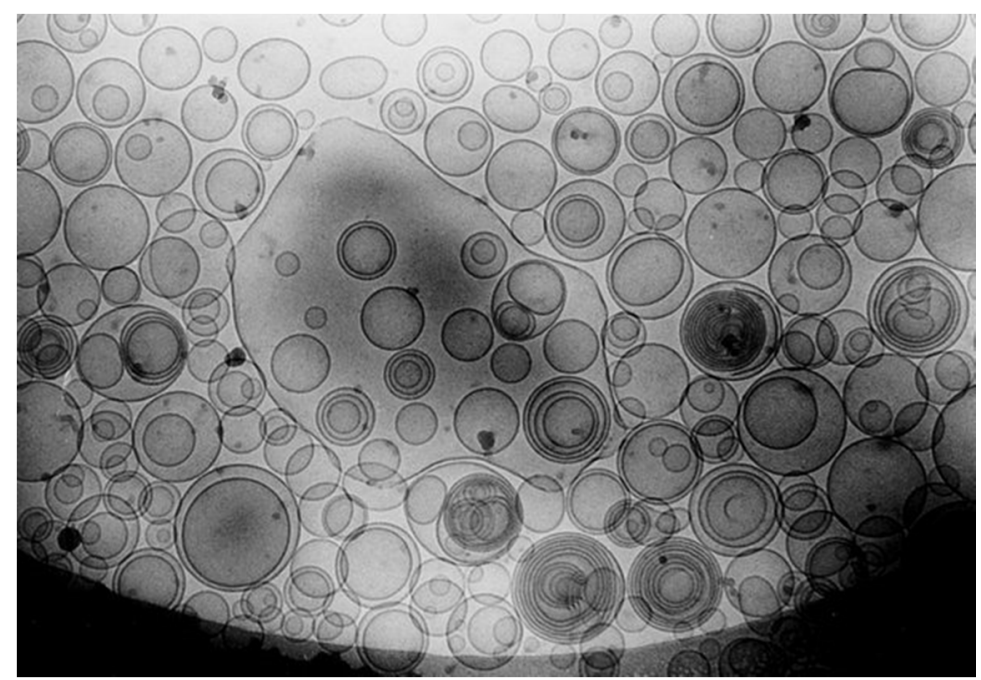

Figure 1.5.8. TEM image of MLVs. ${ }^{232}$ 
Checking the encapsulation efficiency of the liposomes is usually performed by trapping a compound of interest inside the vesicles, removing free compound not in the vesicles by dialysis, and then destroying the vesicles and measuring how much of the desired drug or agent is present as compared to the amount that was initially attempted to load when the vesicles were formed. The volume entrapped in the agent can be determined by loading a fluorescent probe into the vesicles, removing free fluorophore from the solution by dialysis, then freeing the fluorophore and measuring the concentration of the probe. The encapsulation efficiency and entrapped volume methods assume that the liposomes are unilamellar. NMR analysis can also provide information concerning the size of the vesicles if a shift reagent is loaded into the liposomes, free shift reagent is removed by dialysis, and then measuring the area under the two peaks representative of the aqueous medium with and without the shift reagent (Figure 1.5.9).

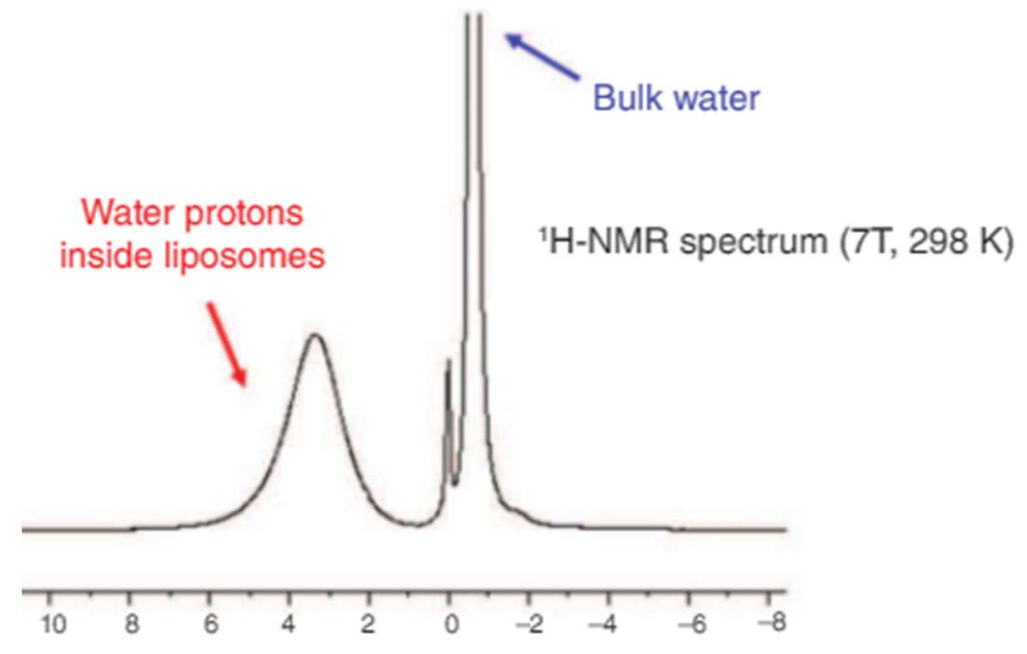

Figure 1.5.9. Intraliposomal NMR analysis. ${ }^{233}$

\subsection{Scope of This Work}

The overall goal of this work was to explore water exchange kinetics and to sow water exchange in two different systems. The first system studied was a rigid $\mathrm{Ln}^{3+} \mathrm{NB}-\mathrm{DOTAM}$ 
chelate with hydrophobic amide substituents. The chelate studied was similar to a previously studied chelate with hydrophilic amide substituents, which allowed for comparison between the two moieties and their effect on the amount of CEST signal that was seen. The second system studied was a phospholipid bilayer system that had various parameters changed to try to optimize the water exchange rate across the membrane. A second vesicle system was minimally studied when the first system failed to produce a slow water exchange rate, however studies on the second system were difficult and parameters were not optimized for the system. 


\section{CHAPTER 2. STRUCTURAL MANIPULATION PROJECT}

\subsection{Isomer Notation}

In general, the notation of chelates used would go as follows:

$$
\text { "corner " } \Lambda(\delta \delta \delta \delta)-S S S S-\text { Axial - Ln4 }
$$

Where:

"Regioisomer from NB Substitution" - Arm Orientation(Ring Orientation)

- $\alpha($ or $\delta)$ Substituent Stereochemistry

- ( $\delta$ Substituent Position, if present $)-\operatorname{Metal}($ Ligand $)$

However, for simplicity, there is a shorter notation that will be used from here forward.

The above chelate will be carried forward as an example and a structure of the chelate is given in Figure 2.1.1 for reference.

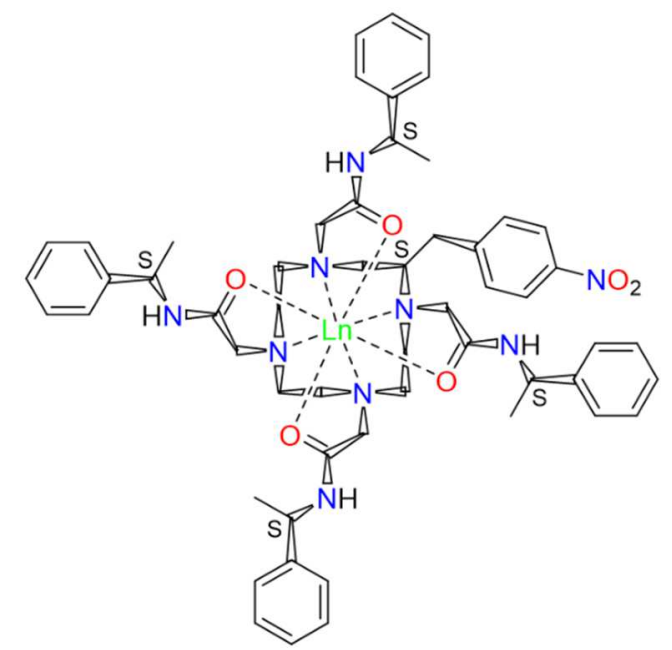

Figure 2.1.1. Reference chelate for naming notation, "corner"- $\Lambda(\delta \delta \delta \delta)-S S S S-A x i a l-L n 4$.

By substituting on the macrocyclic ring with a nitrobenzyl group, the $\lambda \lambda \lambda \lambda$ orientation of the ethylene bridges is locked out due to the pro-S positioning of the substituent and only the $\delta \delta \delta \delta$ orientation is available for chelate formation. Therefore, the (Ring Orientation) notation is removed, to give: 


$$
\text { "corner" - } \Lambda-S S S S-\text { Axial - Ln4 }
$$

The substitution on the macrocyclic ring also leads to two possible regioisomers (Figure 2.1.2), a side regioisomer (denoted by "s") and a corner regioisomer (denoted by "c"). Therefore, the "Regioisomer from NB Substitution" is shortened from the word "corner" to "c", to give:

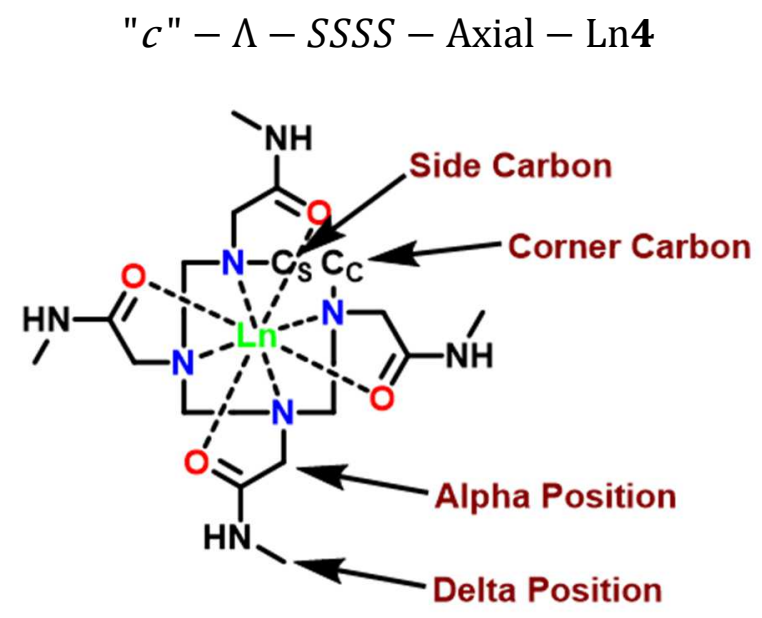

Figure 2.1.2. Substitution positions.

The substitution at the $\alpha$ position on the pendant arm (Figure 2.1.2) controls the coordination geometries, where matching arm and ring orientations lead to TSAP isomers (S@ $@$ gives $\Delta(\delta \delta \delta \delta)$; denoted by $\Delta$ ) and opposing arm and ring orientations lead to SAP isomers ( $\mathrm{R} @ \alpha$ gives $\Lambda(\delta \delta \delta \delta)$; denoted by $\Lambda)$. This means that the Arm Orientation will be left as $\Lambda$ (representative of opposing arm and ring orientations, or SAP isomers), to give:

$$
" c "-\Lambda-S S S S-\text { Axial - Ln4 }
$$

Substitution at the $\delta$ position (Figure 2.1.2) does not halt arm rotations but plays a role in where the arm substituent resides in space. Whether the substituent is found in a pseudoaxial (denoted by Ax) or pseudo-equatorial (denoted by Eq) position (Figure 2.1.3) is dependent on the stereochemical configuration at the $\delta$ position and the helicity of the arms. 
However, the stereochemistry at the $\delta$ position is given by which ligand is used $(\mathbf{1}, \mathbf{2}, \mathbf{3}$, or $4-\mathbf{1}$ and $\mathbf{3}$ have the $R R R R$ stereochemistry at the $\delta$ position and $\mathbf{2}$ and $\mathbf{4}$ have the SSSS stereochemistry at the $\delta$ position). This means that the $\delta$ Substituent Stereochemistry notation SSSS can be removed, to give:

$$
" c "-\Lambda-\text { Axial }-\operatorname{Ln} 4
$$

And the $\delta$ Substituent Position (Figure 2.1.3) can be shortened to Ax (pseudo-axial) or Eq (pseudo-equatorial) to show where the substituent is located in space, to give:

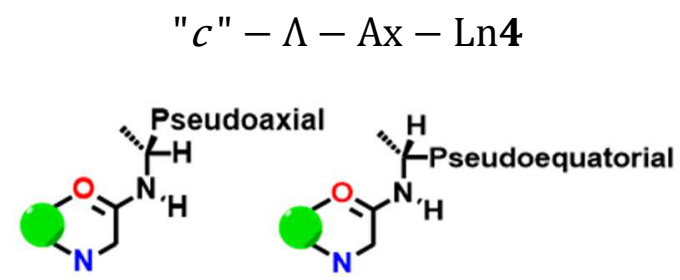

Figure 2.1.3. Pseudoaxial and pseudoequatorial positions in space.

This condensed notation form will be used for all chelates in this chapter, and a flowchart can be found in Figure 2.1.4 and 2.1.5 to help in the identification of isomers discussed.

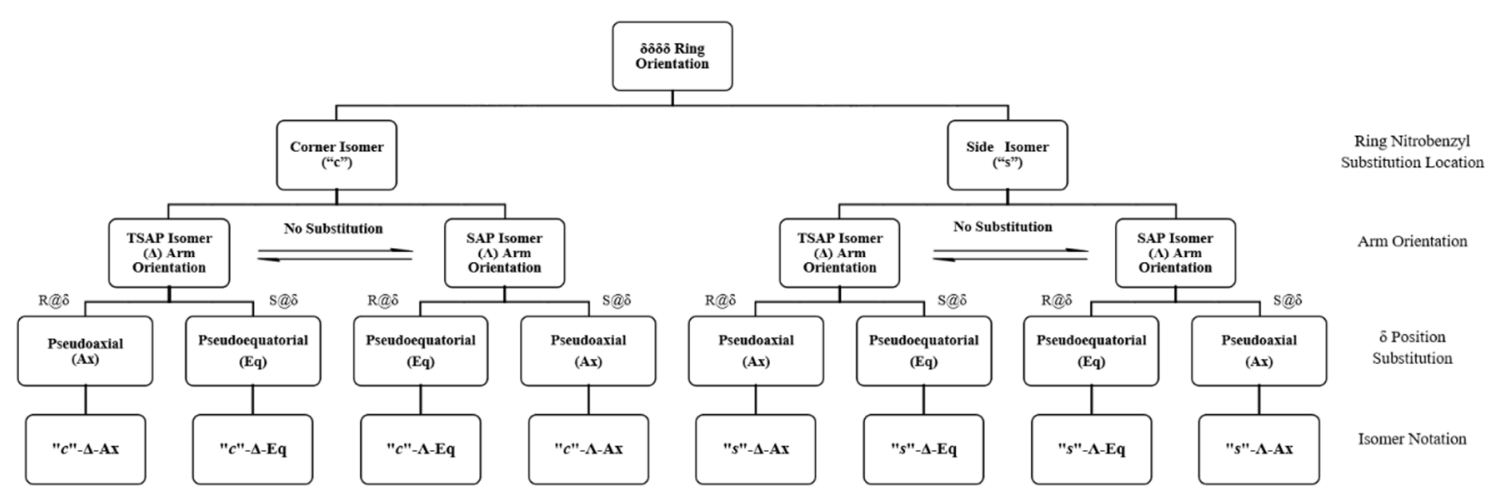

Figure 2.1.4. Isomer naming flowchart.

In order to look at the effect of substituting at the $\delta$ position on isomer distribution, a thorough study on a handful of lanthanide NB-DOTAM chelates was undertaken. Both hydrophilic (carboxylic) and hydrophobic (phenyl) $\delta$ position substituents were studied for 
their effect on water control and CEST effect. The carboxylic chelate studies were performed previously and served as motivation for the phenyl chelate studies. Both types of chelates were substituted on the macrocyclic ring with a nitrobenzyl (NB) substituent to lock out ring inversion conversion between SAP and TSAP isomers, and the chelates were also substituted on the pendant arms with a methyl group at the $\delta$ position in hopes of stopping arm rotation conversion between SAP and TSAP isomers. Both chiralities of the pendant arm substitution were synthesized and therefore both the RRRR and SSSS ligands were investigated. Figure 2.1.5 can serve as a "cheat sheet" for tracking the structures of the chelates $\mathbf{1}, \mathbf{2}, \mathbf{3}$, and $\mathbf{4}$, and for visualizing the results of the experiments, as indicated by the different colored boxes. The flowcharts are included throughout the chapter to help keep isomers straight and to help organize the data and results. 

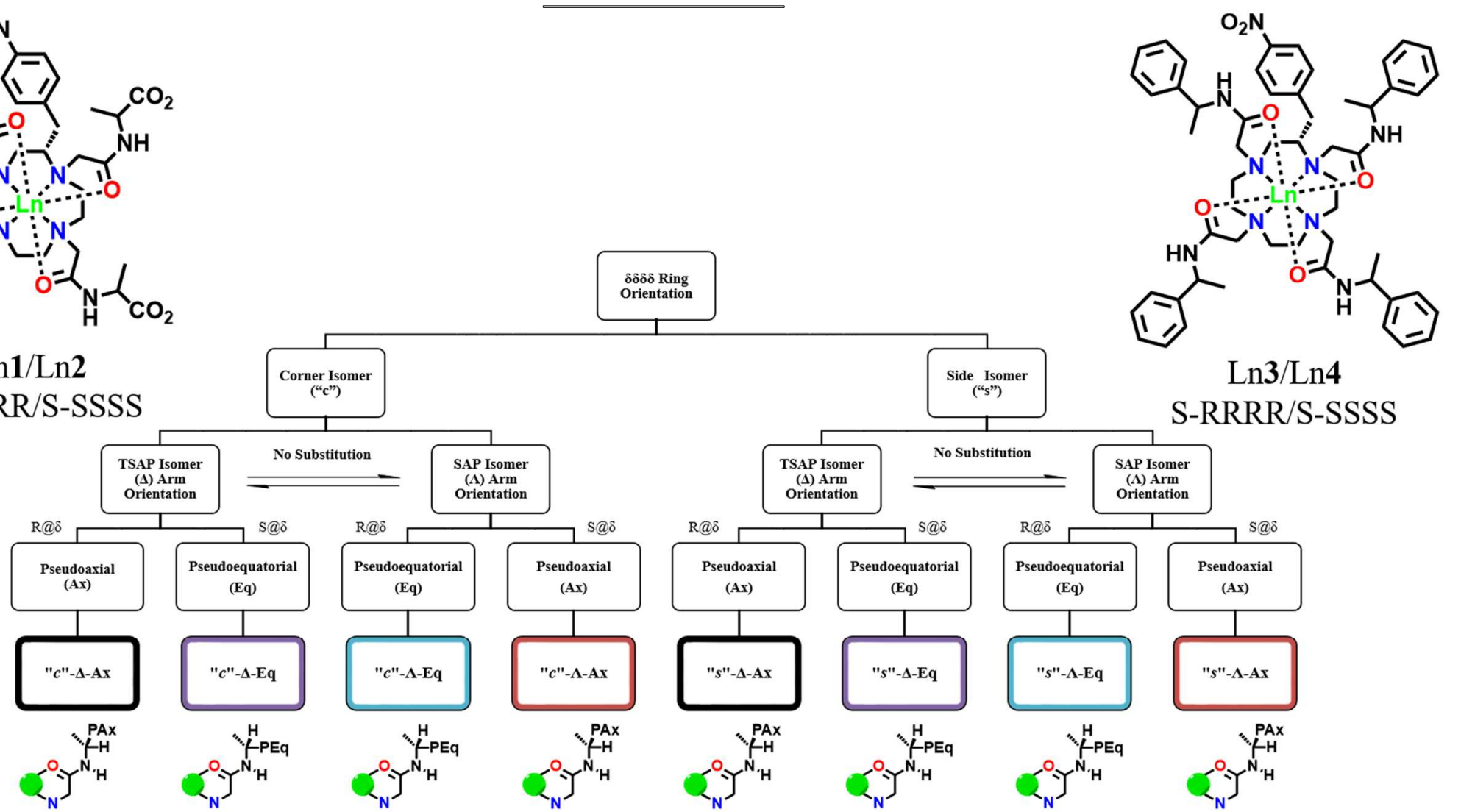

$\operatorname{Ln} 3 / \operatorname{Ln} 4$ S-RRRR/S-SSSS

Figure 2.1.5. Flowchart "cheat sheet". The boxes with bold black outlines represent isomers that were not observed at all in the NMR studies. The boxes with bold blue outlines represent isomers that were observed in the Ln1 and Ln3 chelates. The boxes with bold purple outlines represent the isomers that were observed in the Ln $\mathbf{2}$ and Ln 4 chelates. The boxes with the bold red outlines represent the isomers that grew into the sample as the lanthanide series was crossed in the Ln2 and Ln4 chelates. 


\subsection{Motivation-Carboxylic Derivatives ${ }^{234}$}

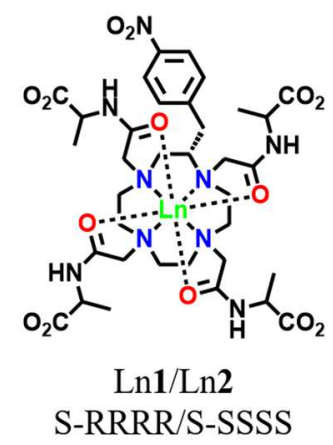

Figure 2.2.1. Carboxylate $\mathrm{Ln}^{3+}$ DOTAM chelates studied.

\section{Basic NMR Studies}
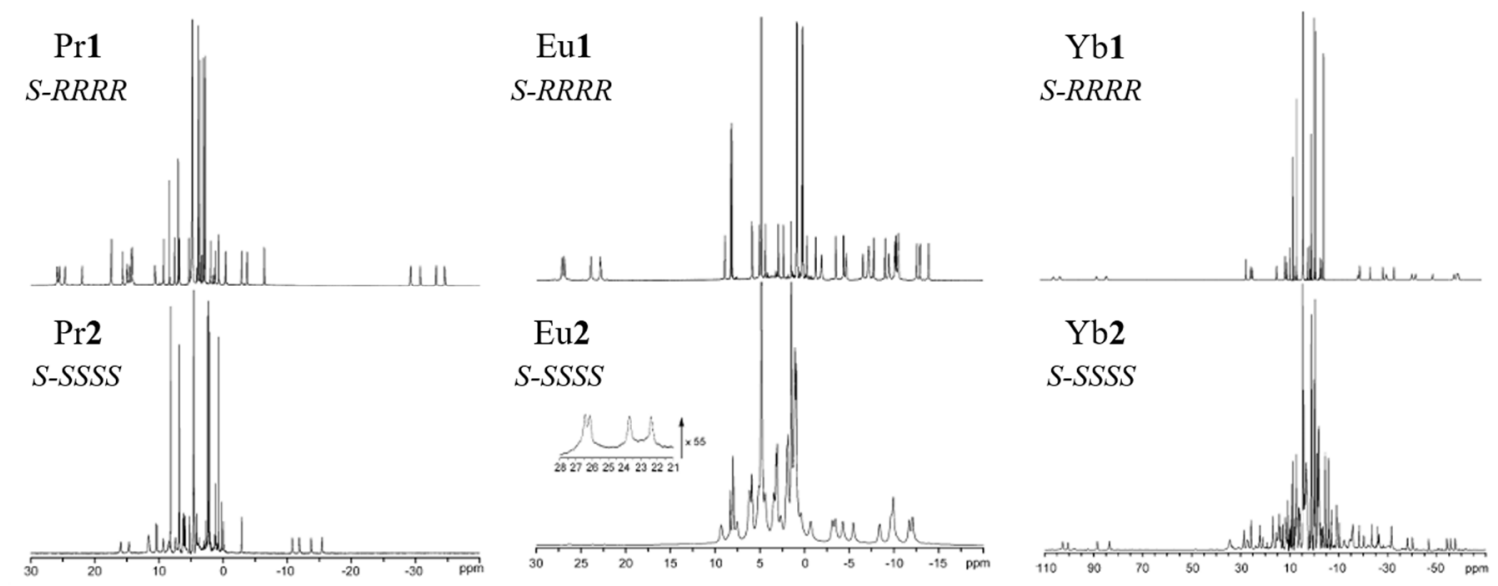

Figure 2.2.2. Ln1/2 ${ }^{1} \mathrm{H}$ NMRs.

Ln1 and Ln2 were chelated with $\mathrm{Pr}^{3+}, \mathrm{Eu}^{3+}$, and $\mathrm{Yb}^{3+}$ ions (Figure 2.2.1). NMRs were taken of all chelates to identify isomers present in solution. Isomers are differentiated in NMR by the shift of their axial protons on the macrocyclic ring. SAP isomers are highly shifted downfield by their proximity to the metal ion. With TSAP isomers, the ligand field is smaller which leads to less shifted axial protons on the macrocyclic ring. When looking at $\mathrm{Ln} 1$ across the series (Figure 2.2.2), only SAP isomers were present in the $\mathrm{Pr}^{3+}, \mathrm{Eu}^{3+}$, and $\mathrm{Yb}^{3+}$ chelates $(\Lambda-\operatorname{Pr} \mathbf{1} / \mathrm{Eu} \mathbf{1} / \mathrm{Yb} \mathbf{1}) . \mathrm{Ln} \mathbf{2}$ showed only TSAP isomer in the $\operatorname{Pr}^{3+}$ chelate $(\Delta-$ Pr2). As the ionic radius of the metal ion complexed with the ligand decreased in size, the 
isomer distribution shifted from pure TSAP isomer to a mix of SAP and TSAP isomer. When looking at the $\mathrm{Eu}^{3+}$ chelate, it appeared that only TSAP isomer was present $(\Delta$-Eu2), but upon closer inspection a small amount of SAP isomer was identified in solution $(\Lambda$ Eu2). The amount of SAP isomer increased with the $\mathrm{Yb}^{3+}$ chelate. This indicated the SAP isomer was preferred for the smaller $\mathrm{Ln}^{3+}$ metal ions found at the end of the lanthanide series, indicating that the coordination cage is smaller for SAP isomers than TSAP isomers, but also indicating that the $\delta$ position substitution on the pendant arm had very little control over arm helicity. The $\delta$ substituent suggested that Ln1 isomers have a low energy geometry (SAP) with the substituent in a low energy position (pseudo-equatorial), giving only the following isomers in each sample: $\Lambda$-Eq-Pr1/Eu1/Yb1. (Figure 2.2.3) However, with the reversed stereochemistry, Ln2 adopted the reversed arm helicity and appeared as a high energy geometry (TSAP) with a low energy substituent position, giving only the $\Delta$ Eq-Pr2 isomer in the beginning of the lanthanide series (Figure 2.2.3). However, moving across the series forced Ln2 to place the substituent in a higher energy position (pseudoaxial) so the geometry could be a lower energy configuration (SAP) for some of the sample, giving the following isomers: $\Delta-\mathrm{Eq}-\mathrm{Eu} 2 / \mathrm{Yb} 2$ and $\Lambda$-Ax-Eu2/Yb2. 


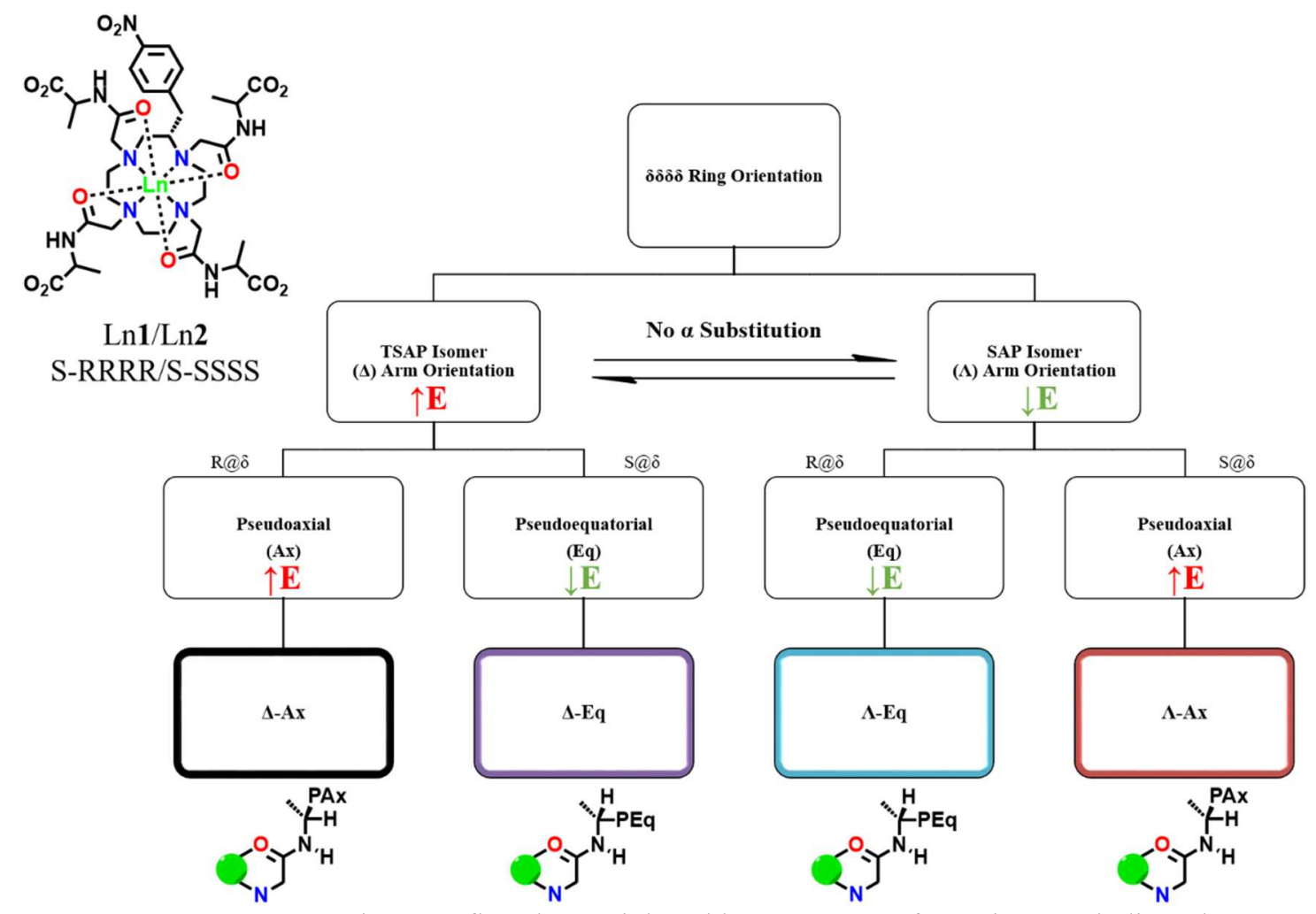

Figure 2.2.3. Ln1/2 NMR flowchart. High and low energy conformations are indicated.

\section{CEST Experiments}

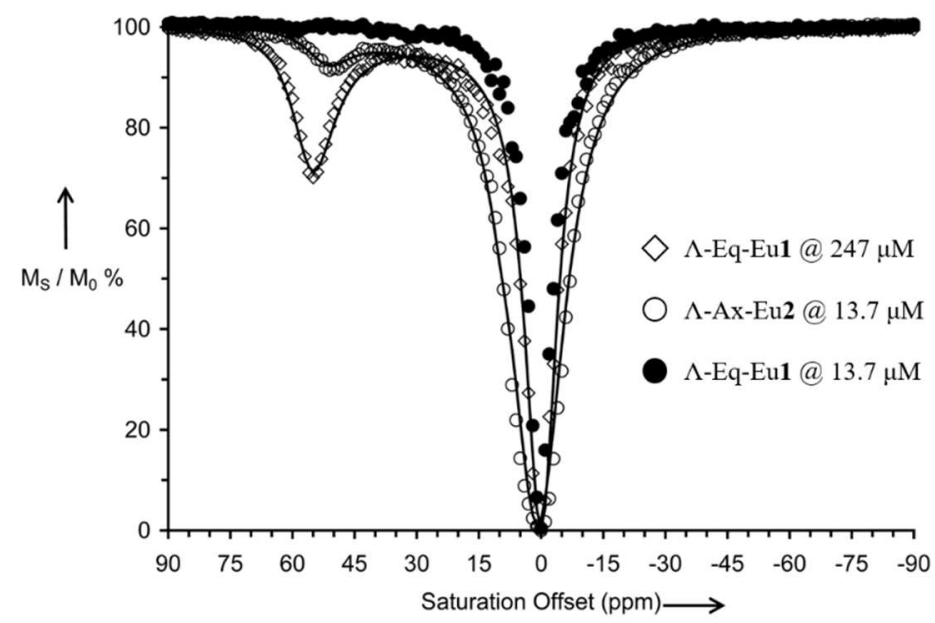

Figure 2.2.4. Eu1/2 CEST spectra.

CEST experiments were performed to explore the water exchange rates of each europium chelate prepared (Figure 2.2.4). The Eu1 and Eu2 chelates had CEST peaks appear, but with varying intensities and only for the SAP isomers $(\Lambda$-Eq-Eu1 and $\Lambda$-Ax- 
Eu2). TSAP isomers don't usually appear in CEST spectra due to their water exchange kinetics being faster than what is ideal for CEST. SAP isomers, having slower water exchange kinetics, do appear in CEST spectra. The open diamonds, the largest CEST peak obtained, represent Eu1 with a $\Lambda$-Eq-Eu2 isomer concentration of $274 \mu \mathrm{M}$. The open circles, the middle CEST peak obtained, represent Eu2 with a $\Lambda$-Ax-Eu2 isomer concentration of $13.7 \mu \mathrm{M}$. The concentration of SAP isomer for Eu1 is much greater in these two cases, therefore a third CEST experiment was run with the concentration of the $\Lambda$-Eq-Eu1 isomer equal to $13.7 \mu \mathrm{M}$ (closed circles). A comparison could then be made between the $\Lambda$-Eq-Eu1 and $\Lambda$-Ax-Eu2 isomers. The $\Lambda$-Eq-Eu1 sample with the concentration of $13.7 \mu \mathrm{M}$ had no effective CEST peak arise in the spectrum. This means that the $\Lambda$-Ax-Eu2 isomer has a water exchange rate appropriate for CEST imaging while the $\Lambda$-Eq-Eu1 isomer does not, which means that the water exchange rate for the $\Lambda$-Ax$\mathrm{Eu} \mathbf{2}$ isomer is comparably slower than that for the $\Lambda$-Eq-Eu1 isomer.

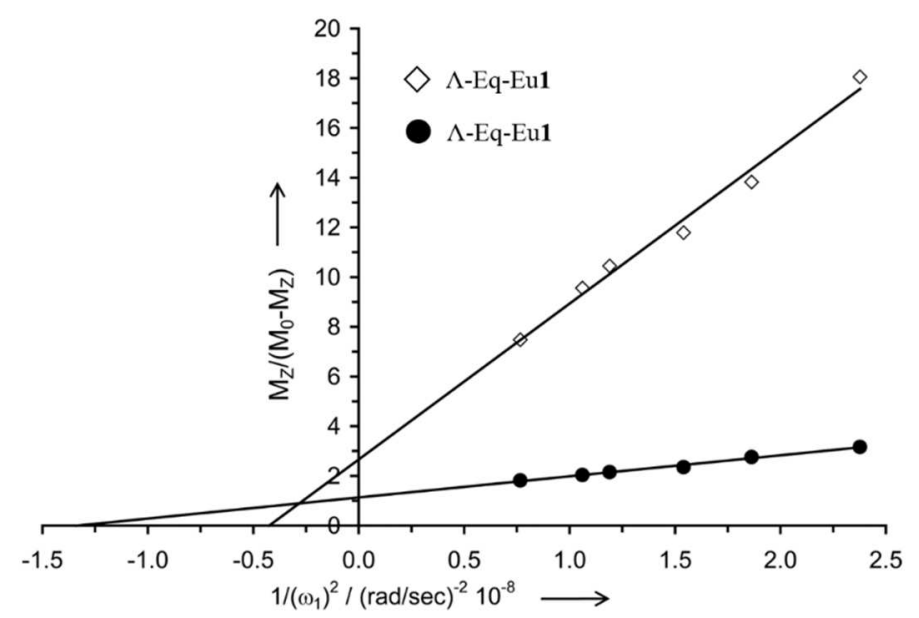

Figure 2.2.5. Eu1/2 Omega plots. Omega plots were used for calculating water exchange rate of the chelates.

However, after omega plots were used to analyze the spectra over varying pulse power levels (Figure 2.2.5), the water residence lifetime for the $\Lambda$-Eq-Eu1 isomer was found to 
be $116 \mu$ s, while the water residence lifetime for the $\Lambda$-Ax-Eu2 isomer was found to be 65 $\mu$ s. Therefore, the $\Lambda$-Eq-Eu1 isomer has a slower water exchange rate, possibly too slow. This suggests that the chirality of the $\delta$ substitution has some effect on the water exchange kinetics seen in the chelates due to the spatial arrangement of the amide substituent (Figure 2.2.6). With the $\Lambda$-Eq-Eu1 isomer, the carboxylic acid group is in a pseudo-equatorial position and has slow water exchange not ideal for CEST imaging. This could be due to the pseudo-equatorial carboxylic acid attracting water away from the water exchange site. Water exchange on and off of chelates is a dissociative mechanism, so if the pool of water to exchange with is low, then the chelate would hold onto the water it is coordinated with longer than normal. If the pool of water near the exchange site was full, then the lanthanide would have no reason to hold onto the coordinated water for as long since another water would quickly replace the one it would lose. The $\Lambda$-Ax-Eu2 isomer has the amide substituent in a pseudo-axial position, and presumably has a larger pool of water molecules near the exchange site, making the water exchange rate speed up to a rate that is ideal for CEST imaging. 


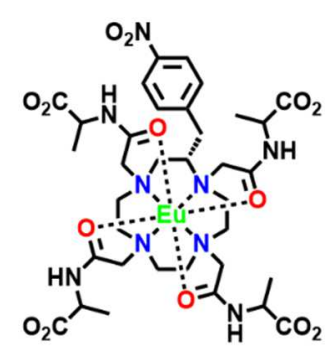

Eu1/Eu2 S-RRRR/S-SSSS
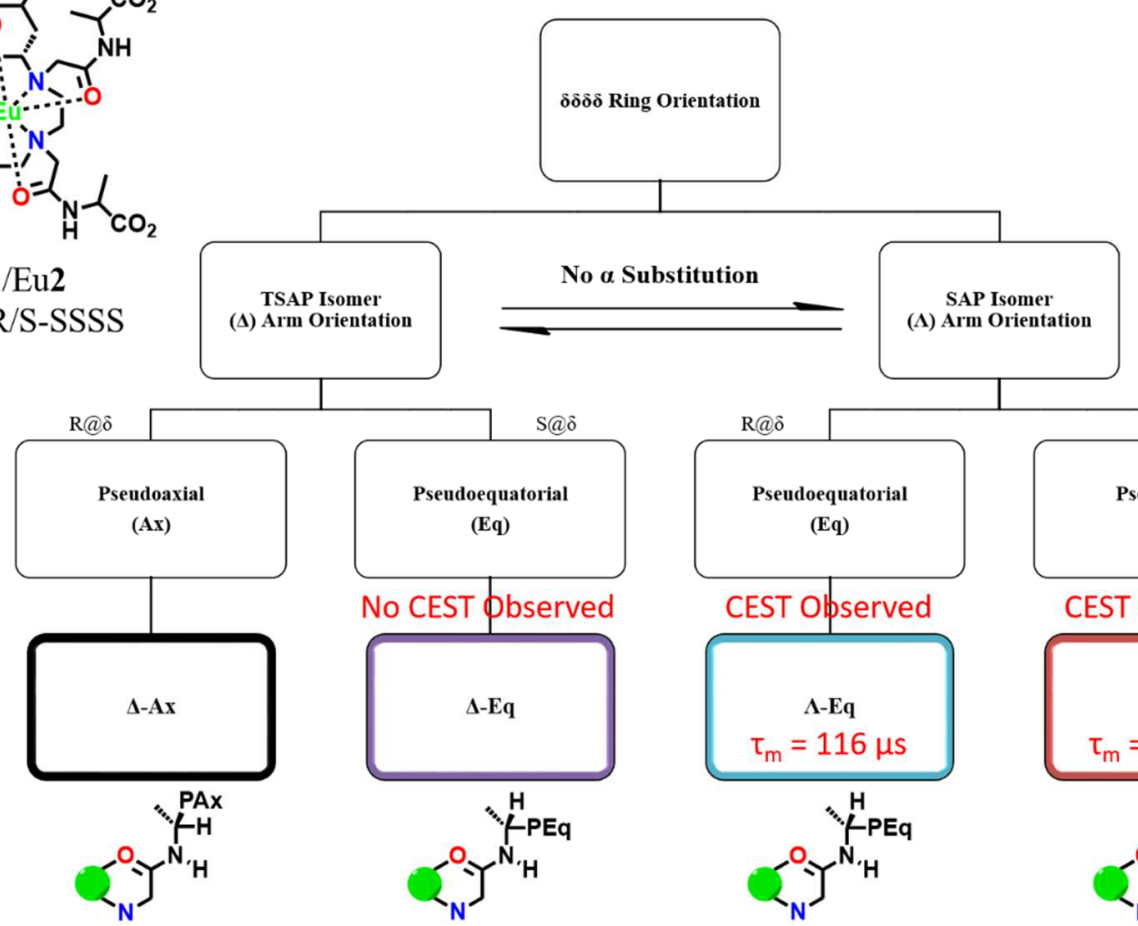

Figure 2.2.6. Eu1/2 CEST flowchart. Found $\tau_{\mathrm{m}}$ values are indicated.

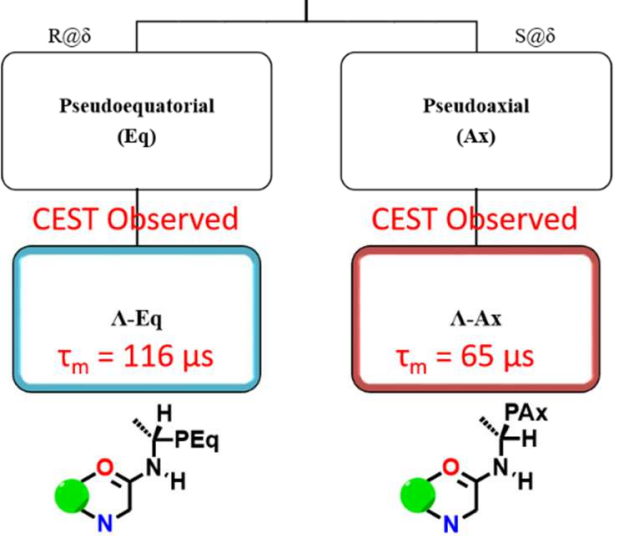

The spatial arrangement could be affecting the water exchange rates for the $\Lambda-A x-E u 2$ isomer by creating a pool of water around the hydrophilic groups, which would then exchange with the chelate and put affected water molecules back into the pool around the substituents. The exchange between the pool around the substituents and the bulk water in the sample could be slow in the $\Lambda$-Ax-Eu2 isomer due to the attraction of the water to the hydrophilic amide groups, creating an artificially slow water exchange rate even though the pool around the exchange site is larger, which could be giving the $\Lambda$-Ax-Eu 2 isomer its impressive CEST signal. This hypothesis lead to the next NB-DOTAM chelate studied to have hydrophobic amide groups instead of hydrophilic groups. 


\subsection{Studied Chelates - Phenyl Derivatives ${ }^{235}$}

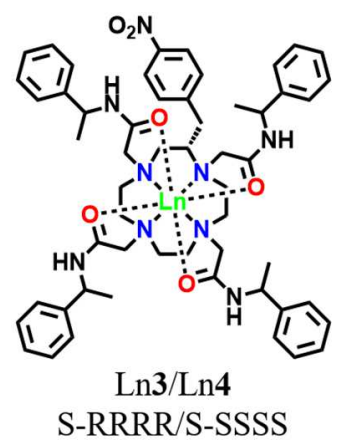

Figure 2.3.1. Phenyl $\mathrm{Ln}^{3+}$ DOTAM chelates studied.

\section{Basic NMR Studies}

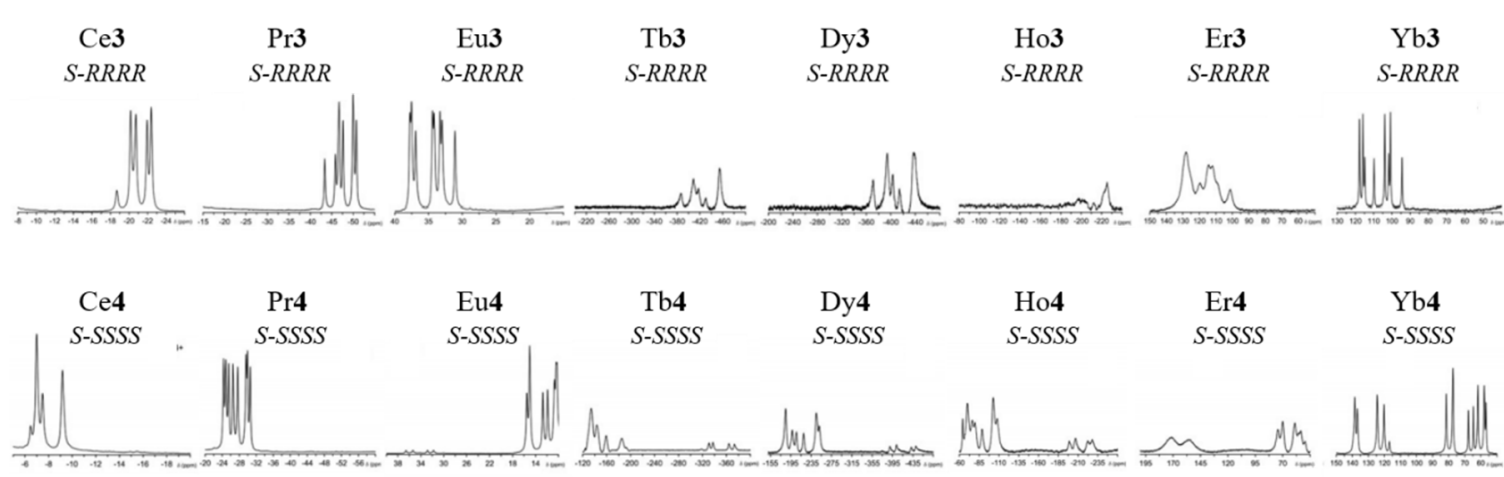

Figure 2.3.2. Ln 3/4 ${ }^{1} \mathrm{H}$ NMRs. The scale follows conventional NMR convention where downfield is to the left.

Ln3 and Ln4 were chelated with $\mathrm{Ce}^{3+}, \mathrm{Pr}^{3+}, \mathrm{Nd}^{3+}, \mathrm{Sm}^{3+}, \mathrm{Eu}^{3+}, \mathrm{Tb}^{3+}, \mathrm{Dy}^{3+}, \mathrm{Ho}^{3+}, \mathrm{Er}^{3+}$, and $\mathrm{Yb}^{3+}$ ions (Figure 2.3.1). Isomers are differentiated in NMR by the shift of their axial protons on the macrocyclic ring. SAP isomers are highly shifted downfield by their proximity to the metal ion. With TSAP isomers, the ligand field is smaller which leads to less shifted axial protons on the macrocyclic ring. Ln3 chelates all adopted the SAP geometry $(\Lambda$-Eq-Ln3) with no change of coordination geometry as the ionic radius of the lanthanide ion decreased, just like the Ln1 chelates (Figure 2.3.2). However, it was noted this time around that two different regioisomers were present in solution due to the nitrobenzyl substituent being present on both the side and corner position of the 
macrocyclic ring, giving " $s$ "- $\Lambda$-Eq-Ln3 and " $c$ "- $\Lambda$-Eq-Ln3 isomers. Ln4, as seen with $\operatorname{Ln} 2$, started with all TSAP geometry $(\Delta$-Eq-Ln4) present in the early lanthanides, but as the ionic radius decreased across the lanthanide series the amount of SAP coordination geometry $(\Lambda$-Ax-Ln4) present in the samples increased and the ratio between SAP isomers and TSAP isomers increased (Figure 2.3.2). Again, the emergence of SAP isomers in the Ln4 chelates supports the idea that the $\delta$ position substitution does not control which isomer is formed in solution and does not lock arm rotations to cease interconversion between isomers.

As seen with the Ln1 and Ln2 isomer trends, the $\delta$ substituent suggested that Ln3 isomers have a low energy geometry (SAP) with the substituent in a low energy position (pseudo-equatorial), giving only the following isomers in each sample: $\Lambda$-Eq-Ln3 (Figure 2.3.3). However, with the reversed stereochemistry, Ln4 adopted the opposite arm helicity and appeared as a high energy geometry (TSAP) with a low energy substituent position, giving only the $\Delta$-Eq-Ln4 isomer with the early lanthanides (Figure 2.3.3). Moving across the series forced the Ln4 chelate to place the substituent in a higher energy position (pseudo-axial) so the geometry could be a lower energy configuration (SAP) for some of the sample, giving the following isomers: $\Delta$-Eq-Ln4 and $\Lambda$-Ax-Ln4 for the latter half of the lanthanides used. Not observed with the Ln2 isomers though, were the presence of regioisomers. Both side and corner regioisomers were observed in the Ln 4 chelates (similar to the Ln3 chelates), giving the following 4 different isomers present in solution starting around Eu4 and continuing through the end of the lanthanide series: " $s$ "- $\Delta$-Eq-Ln4 and " $c$ "$\Delta$-Eq-Ln4, and " $s "-\Lambda$-Ax-Ln4 and " $c$ "- $\Lambda$-Ax-Ln4 isomers (Figure 2.3.3). 


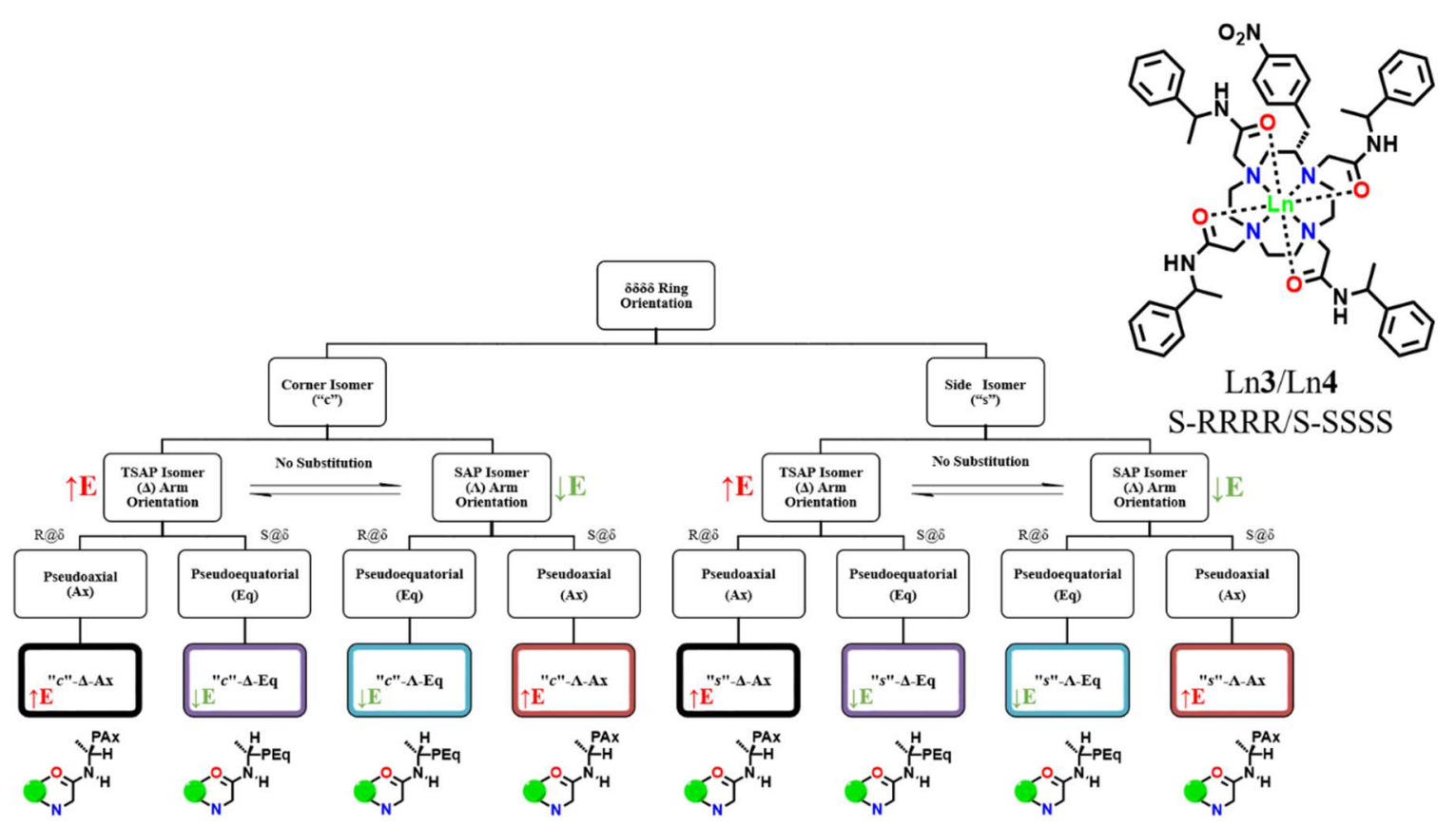

Figure 2.3.3. Ln3/4 NMR flowchart. High and low energy conformations are indicated for the isomers.

The presence of both side and corner isomers required that the peaks found in the NMRs be identified, which were identified using variable temperature and twodimensional correlation spectroscopy experiments.

\section{Variable Temperature Experiments}

Variable temperature experiments were performed on the Yb4, Eu3, and Eu4 samples. Since temperature and induced shifts are inversely proportional, it was expected, and observed, that as the temperature increased the induced shifts decreased. This is best seen in the axial protons of the chelates. Each chelate has 4 axially located protons on the ethylene bridges between the nitrogen donor atoms in the macrocyclic ring. The axially located protons are shifted based on their location to the metal. SAP isomer axial protons are shifted further downfield than TSAP isomer axial protons. If one SAP isomer were present in solution, there would be 4 highly shifted axial protons, and if two SAP isomers are present in solution, there would be 8 highly shifted axial protons. The same is true for 
TSAP isomers. So in total, the Ln4 samples that contain all four isomers ("c"- $\Delta$-Eq, " $c$ "$\Lambda-\mathrm{Ax}$, " $s "-\Delta-\mathrm{Eq}$, and " $s$ "- $\Lambda-\mathrm{Ax})$ should have 16 total axial protons in their ${ }^{1} \mathrm{H}$ NMRs, 8 highly shifted SAP isomer axial protons and 8 less shifted TSAP isomer axial protons.

The Yb4 sample contained the " $c$ "- $\Delta$-Eq, " $c$ "- $\Lambda-A x$, " $s$ "- $\Delta-\mathrm{Eq}$, and " $s$ "- $\Lambda-A x$ isomers. The variable temperature data (Figure 2.3.4) showed more than 4 signals for both the TSAP and SAP axial protons, meaning that both the TSAP (" $c$ "- $\Delta$-Eq and " $s$ "- $\Delta$-Eq) and both the SAP ("c"- $\Lambda$-Ax and " $s "-\Lambda-A x)$ isomers were present in solution. As the temperature was increased, the " $c$ " $-\Delta$-Eq isomer and " $c$ "- $\Lambda$-Ax isomer and the " $s$ "- $\Delta$-Eq isomer and " $s$ "- $\Lambda$ Ax isomer that were in exchange through arm rotations moved toward each other, showing that they were in exchange.

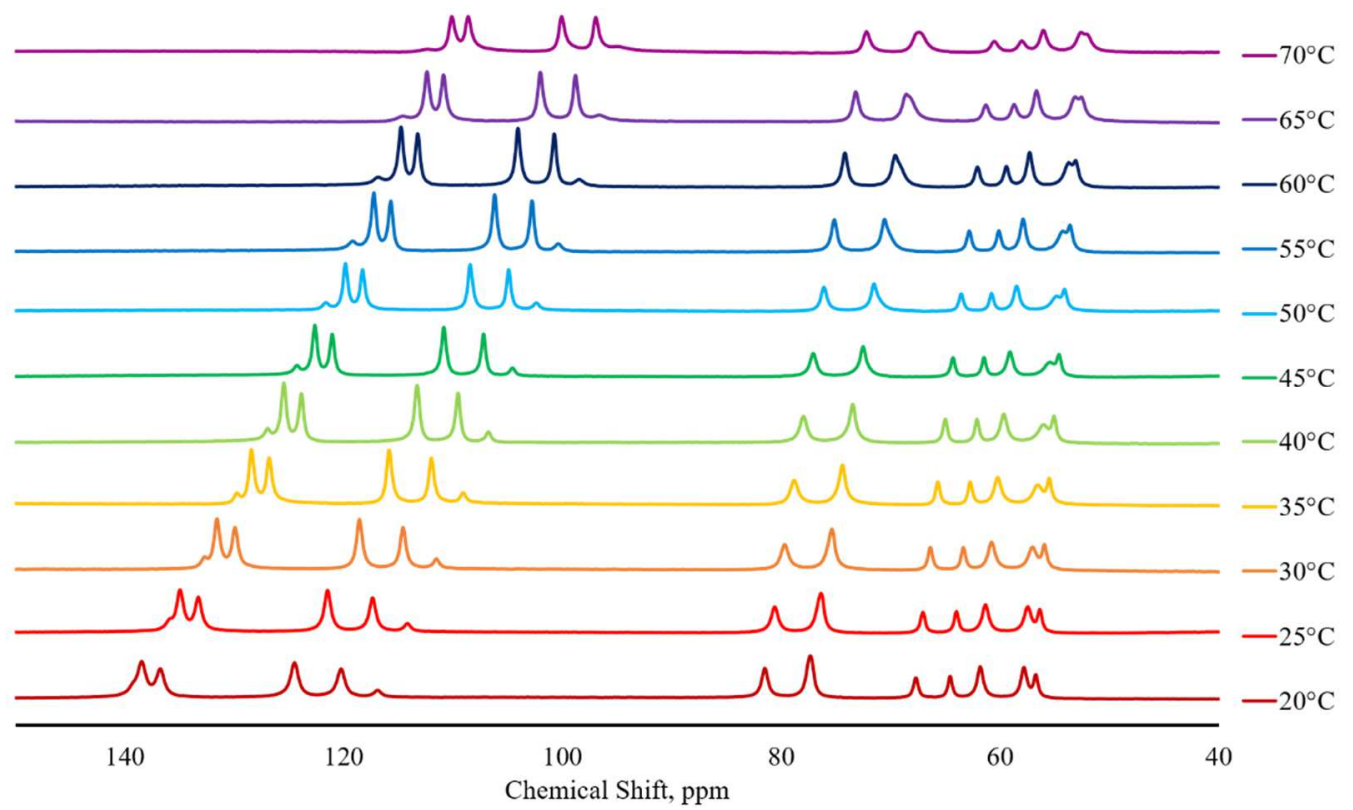

Figure 2.3.4. Yb4 variable temperature experimental data. The most shifted axial protons belong to the SAP isomers " $c$ "- $\Lambda-A x$ and " $s "-\Lambda-A x$, and the less shifted axial protons belong to the TSAP isomers " $c$ "$\Delta$-Eq and " $s "-\Delta$-Eq.

The Eu3 sample contained only the "c"- $\Lambda-E q$ and "s"- $\Lambda$-Eq isomers. More than 4 signals were observed as highly shifted axial protons and no signals were observed as 
TSAP axial protons (Figure 2.3.5). The two SAP isomers did not move toward each other as temperature was increased. This was expected since the corner and side isomers are not in exchange with one other. The rate of decreasing induced shift seemed similar between the two isomers, with the "c"- $\Lambda$-Eq isomer rate decreasing slightly faster than the "s"- $\Lambda$ Eq isomer rate.

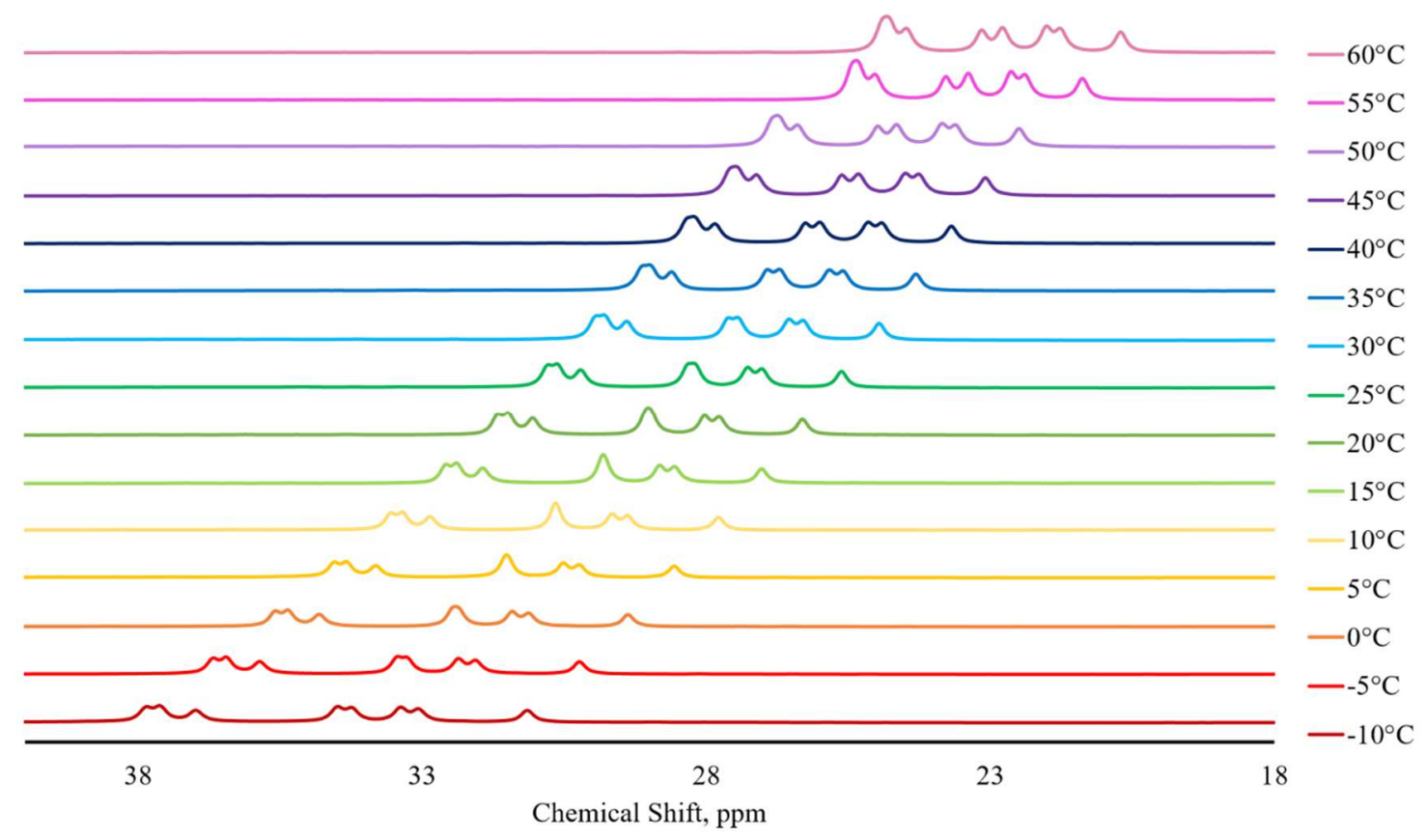

Figure 2.3.5. Eu3 variable temperature data. The axial protons belong to the SAP isomers "c"- $\Lambda$-Eq and "s"- $\Lambda$-Eq.

The Eu4 sample contained the " $c "-\Delta-E q$, " $c "-\Lambda-A x, " s "-\Delta-E q$, and " $s "-\Lambda-A x$ isomers. The sample was comprised of predominately the " $c$ "- $\Delta$-Eq and " $s$ "- $\Delta$-Eq isomers but also had a small amount of converted " $c$ "- $\Lambda$-Ax or " $s "-\Lambda-A x$ isomer present. The TSAP axial protons had more than 4 signals in the ${ }^{1} \mathrm{H}$ NMRs taken, meaning that both the " $c$ "- $\Delta$-Eq and " $s$ "- $\Delta-E q$ isomers were present (Figure 2.3.6). 


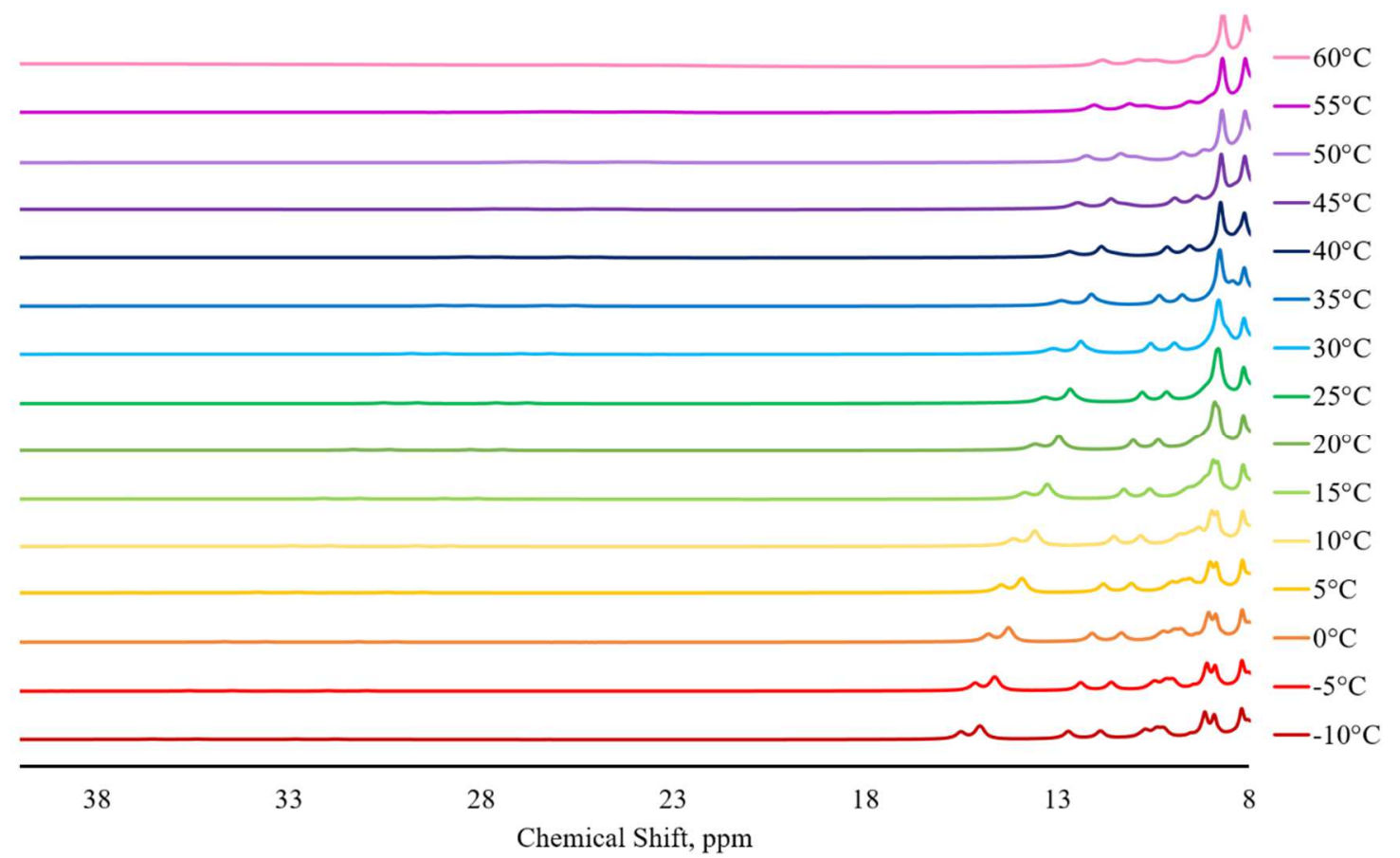

Figure 2.3.6. Eu4 variable temperature data. The axial protons visible on the right belong to the TSAP isomers "c"- $\Delta$-Eq and "s"- $\Delta$-Eq.

In order to visualize the SAP isomer axial protons, the spectra needed to be zoomed in. When the SAP axial protons were visualized, there were only 4 signals present, meaning that the sample had either the " $c$ "- $\Lambda-A x$ or the " $s "-\Lambda-A x$ isomer present, not both (Figure 2.3.7). As the temperature increased, the SAP isomer and the TSAP isomer it was in exchange with moved closer together in the spectrum, slowing the rate of decreasing induced shift in the related TSAP isomer. The four peaks representative of the SAP isomer were present in a two by two pattern, or two sets of doublets. This pattern is the shifting pattern for one of the regioisomers, either the " $c$ "- $\Lambda$-Ax or the " $s$ "- $\Lambda$-Ax isomer. 


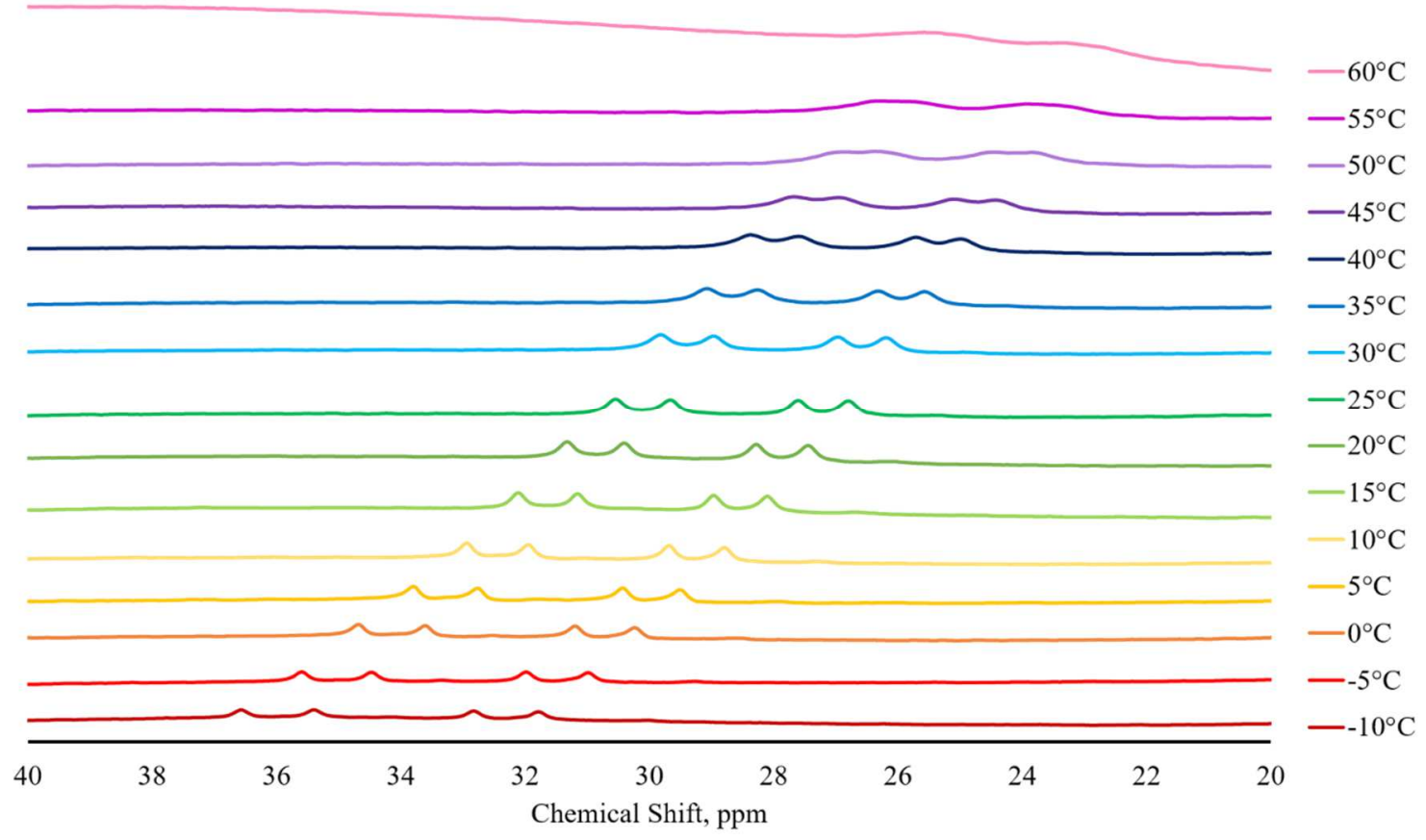

Figure 2.3.7. Zoomed Eu4 variable temperature data. The axial protons visible belong to the SAP isomer, either "c"- $\Lambda$-Ax or "s"- $\Lambda$-Ax.

The variable temperature experiments allowed for visualization of exchanging SAP and TSAP isomers, but identification of the regioisomers wasn't possible until the COSY experiment was run.

\section{COSY Experiments}

The homonuclear correlation spectroscopy (COSY) experiment is used to identify spins which are coupled to each other. In this experiment, a single isotope was looked at: hydrogen. Specifically, the axial protons for the SAP and TSAP isomers were focused on in order to identify side and corner regioisomers. The COSY spectra were obtained on Eu3 and Eu4 also offered insight into how the coordination cage distorts in these specific chelates. 

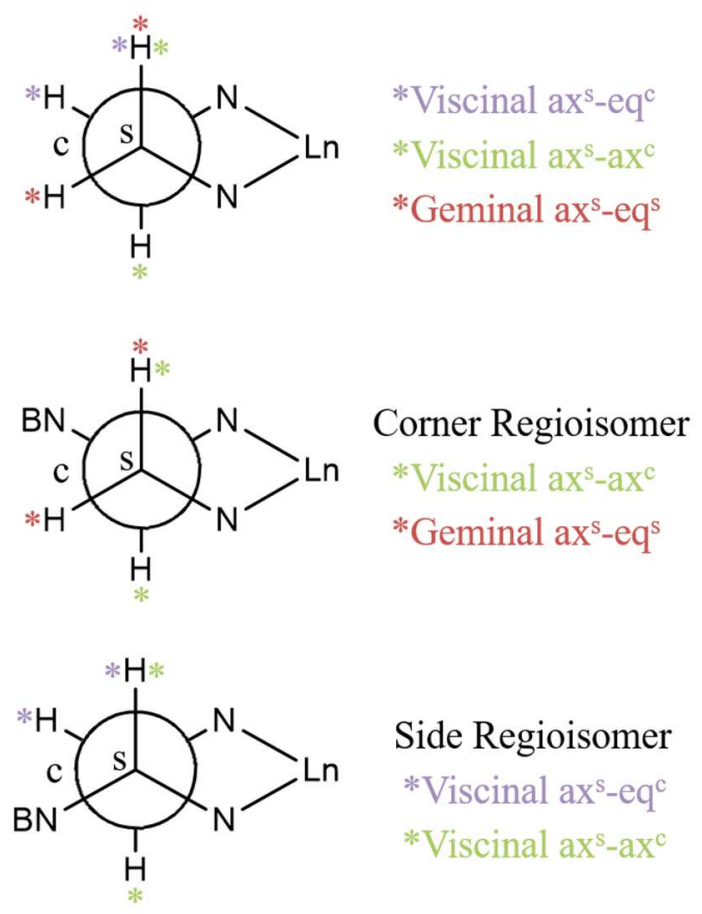

Figure 2.3.8. COSY couplings. Top: The three couplings available to the axial proton on an unsubstituted ethylene bridge. Center: The two couplings available to the axial proton on a corner substituted ethylene bridge. Bottom: The two couplings available to the axial proton on a side substituted ethylene bridge.

An axial proton on an ethylene bridge has 3 other protons near it to couple to. The strongest coupling that can occur is geminal coupling, which occurs between an axial proton and an equatorial proton on the same carbon. The second strongest coupling that can occur is vicinal coupling between the axial proton on one carbon and the axial proton on the neighboring carbon (Figure 2.3.8). The weakest coupling that can occur is vicinal coupling between an axial proton on one carbon and the equatorial proton on the neighboring carbon. On an unsubstituted carbon, the axial proton will have 3 coupling peaks on a COSY spectrum. If an ethylene bridge has a substitution on one of the carbons, the substituent will reside in an equatorial position, which then leaves the axial proton with 2 other hydrogens near it to couple to. If the substituent is on the same carbon as the axial proton, then there will be no geminal coupling in a COSY spectrum, only vicinal coupling 
between the 2 axial protons and vicinal coupling between the axial and neighboring equatorial proton (Figure 2.3.8). If the substituent is on the neighboring carbon as the axial proton, then there will be no vicinal coupling between the axial and neighboring equatorial proton in a COSY spectrum, only geminal coupling and vicinal coupling between the 2 axial protons (Figure 2.3.8). Using this logic, the axial protons associated with the side and corner regioisomers were identified.

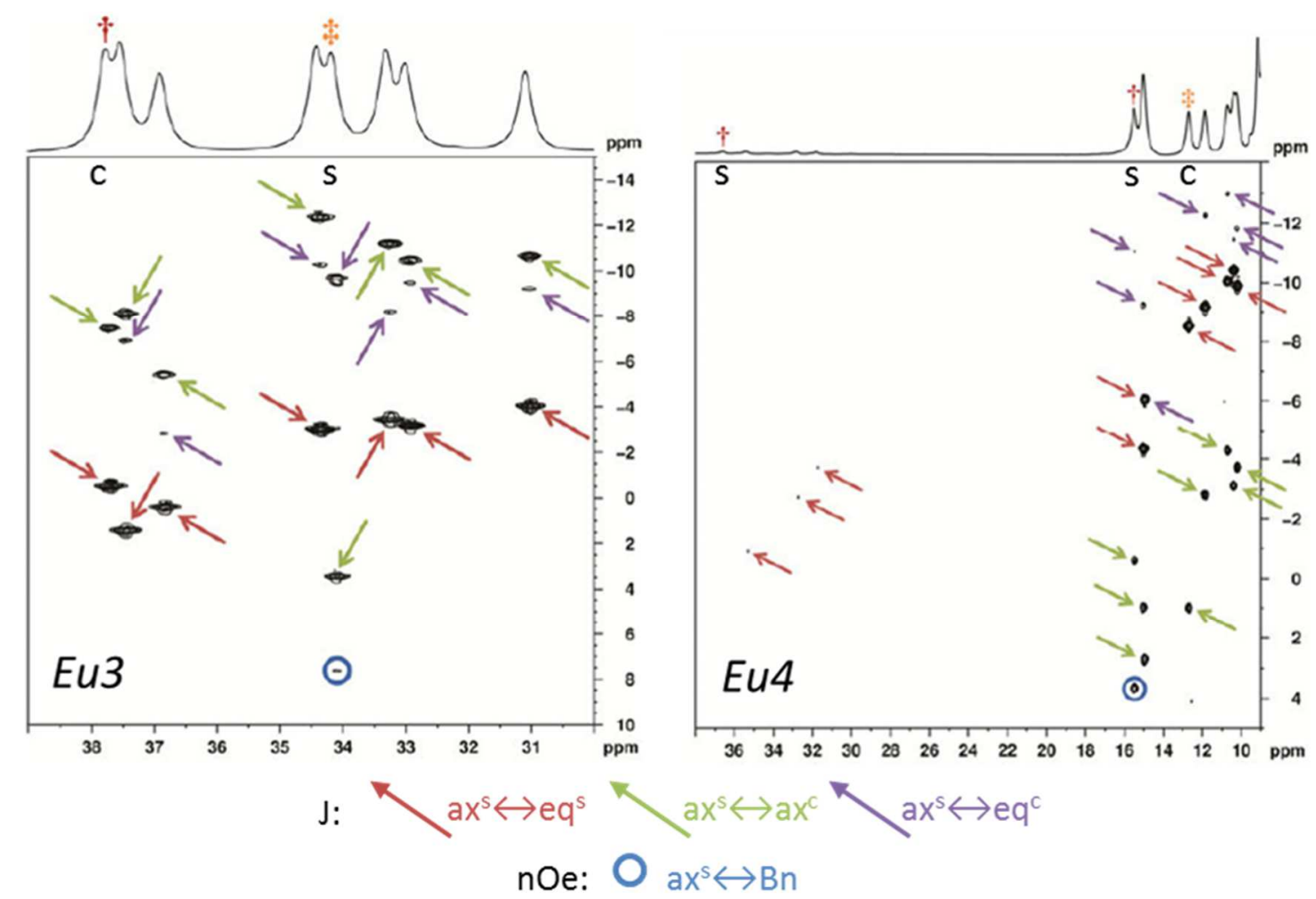

Figure 2.3.9. Eu3/4 COSY spectra. The arrows pointing to each coupling are color coded to identify the type of coupling. The resonances identified as corner or side have red crosses and orange double crosses above them to indicate which resonance was analyzed, and the ending identity is labelled underneath the resonance.

The first, and easiest, step in identifying protons next to a substitution was to count the number of couplings associated with each peak. In the Eu3 sample (Figure 2.3.9), all but two resonances had 3 coupling peaks. The two that didn't have 3 coupling peaks had 2 and by looking at the intensities of the coupling peaks, the second step, it was possible to 
determine which resonance belonged to the axial proton on the corner isomer and which resonance belonged to the axial proton on the side isomer. The most shifted resonance with 2 coupling peaks, near $38 \mathrm{ppm}$, had a strong coupling peak and an intermediate coupling peak, which meant that the axial proton was missing the weakest coupling, the axial proton on one carbon coupling to the equatorial proton on the neighboring carbon. The most shifted resonance was an axial proton on the carbon next to the substitution and, as such, meant that the isomer was the corner regioisomer. Therefore, the most shifted axial proton in the ${ }^{1} \mathrm{H}$ NMRs taken belonged to the " $c$ " $\Lambda$-Eq isomer. The second resonance that had 2 coupling peaks, near 34 ppm, had what looked like two intermediate coupling peaks, which meant that the axial proton was missing the strongest coupling, geminal coupling between the axial proton and the equatorial proton on the same carbon. The second resonance with 2 coupling peaks was an axial proton on the carbon that had the substitution on it and, as such, meant that the isomer was the side regioisomer. Therefore, the axial proton resonance at 34 ppm belonged to the " $s$ "- $\Lambda$-Eq isomer.

In the Eu4 sample (Figure 2.3.9), the SAP isomer only had 1 coupling peak per resonance. Since 2 resonances were missing, it was concluded that the SAP coupling peaks were the strongest coupling signals for those protons, meaning they were geminal couplings. However, there were only 3 present in the spectrum, with the most shifted resonance not having any coupling peaks. With the resonance missing the geminal coupling between the axial proton and the equatorial proton on the same carbon, this resonance belonged to the axial proton on the carbon with the substitution, or the side regioisomer. Therefore, the SAP isomer present in the Eu4 sample was the " $s$ "- $\Lambda$-Ax isomer. The TSAP 
isomer resonances near $16 \mathrm{ppm}$ and $13 \mathrm{ppm}$ were missing the strongest and weakest couplings, respectively. Therefore, the resonance at $16 \mathrm{ppm}$ belonged to the axial proton on the carbon with the substitution, or the side regioisomer, and the resonance at $13 \mathrm{ppm}$ belonged to the axial proton on the carbon neighboring the substitution, or the corner regioisomer. The TSAP isomer with the $16 \mathrm{ppm}$ resonance was the " $s$ "- $\Delta$-Eq isomer and the TSAP isomer with the $13 \mathrm{ppm}$ resonance was the " $c$ "- $\Delta$-Eq isomer.

With $\operatorname{Ln} 3$, the proton located on the side carbon with the nitrobenzyl substituent had a strong vicinal $\mathrm{ax}^{\mathrm{s}}-\mathrm{eq}^{\mathrm{c}}$ coupling, stronger than any of the other $\mathrm{ax}^{\mathrm{s}}-\mathrm{eq}^{\mathrm{c}}$ vicinal couplings observed on the other ethylene bridges (Figure 2.3.9). This strong coupling means that the macrocyclic ring was distorting the torsion angle $(\Phi)$ between the $\mathrm{ax}^{\mathrm{s}}$ and $\mathrm{eq}^{\mathrm{c}}$ protons away from the normal gauche conformation that should be observed, with the angle becoming smaller than what is should have been (Figure 2.3.10). With Ln1, the coupling for the proton located on the side carbon with the nitrobenzyl substituent was smaller, not larger like in Ln3, which means that the angle in the Ln1 sample was being increased, not decreased like in the Ln3 sample. The only difference between $\operatorname{Ln} 3$ and $\operatorname{Ln} 1$ is the amide substituent; Ln 3 contained the phenyl substituent (a hydrophobic group) and Ln 1 contained the carboxylic acid substituent (a hydrophilic group). Ln4 also had a distortion of the macrocyclic ring away from the gauche conformation but in the opposite direction of Ln3. The side regioisomer of Ln4 had weaker vicinal $\mathrm{ax}^{\mathrm{s}}-\mathrm{eq}^{\mathrm{c}}$ coupling, meaning that the angle was larger than ideal, similar to the coupling found in the COSY of Ln1, not stronger with a smaller angle like the Ln3 sample had (Figure 2.3.9). The electronic effects of the substituents could be playing a role in the coordination cage structure but the 
stereochemical configuration of the delta substituent seems to be playing a bigger role in affecting the direction of the distortion.

\section{Ideal Gauche Conformation}
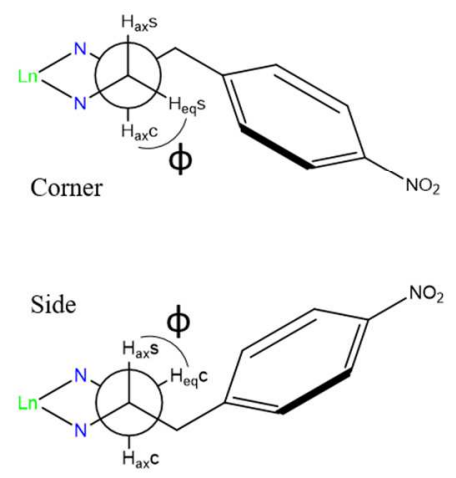

Distortions from Ideal

Gauche Conformation

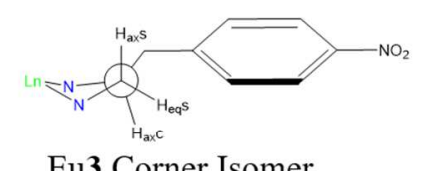

Eu3 Corner Isomer

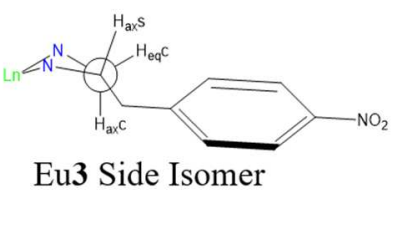

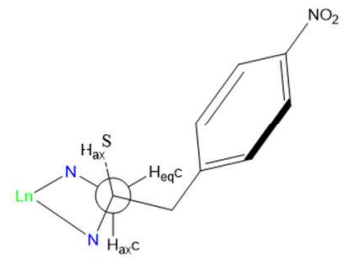

Eu4 Side Isomer

Figure 2.3.10. Substituted carbon gauche conformations. Left: Ideal gauche conformation. Right: Observed gauche conformations in side and corner isomers based on COSY analysis.

In the case of the hydrophobic substituent, it is possible that the ring substituent was aligning with the pseudo-equatorial phenyl derivative through pi bond interactions. This suggested explanation also supports why only SAP isomers are present in the Eu3 samples. The interaction between the ring substituent and the delta position substituent could help to deter arm rotations which would interconvert between the SAP and TSAP isomers.

\section{Differentiating Regioisomers}

After identifying side and corner resonances in the COSY experiments, the variable temperature experimental data was revisited and a shifting pattern for side and corner regioisomers was identified. By tracing the axial protons and their movement as the temperature was increased, patterns in the shifting of the protons became apparent (Figure 2.3.11). In the Eu4 sample, specifically, the " $s$ "- $\Lambda$-Ax SAP isomer was in exchange with the " $s$ "- $\Delta$-Eq TSAP isomer. Because of the exchange process, the resonances belonging to the " $s "-\Delta$-Eq isomer shifted at a different rate than the " $c$ "- $\Delta$-Eq resonances would. By 
observing which resonances in the variable temperature spectra did not shift upfield as quickly, 4 resonances were identified as the axial protons of the side regioisomer, leaving the 4 other resonances to belong to the corner regioisomer. The " $s "-\Lambda-A x$ and " $s "-\Delta-E q$ isomers had similar shifting patterns for their axial protons and the "c" $-\Delta-E q$ isomer had a different shifting pattern for its axial protons. The shifting pattern that appears to hold across the series is a 2-2 shifting pattern for a side isomer and a 1-2-1 shifting pattern for a corner isomer.
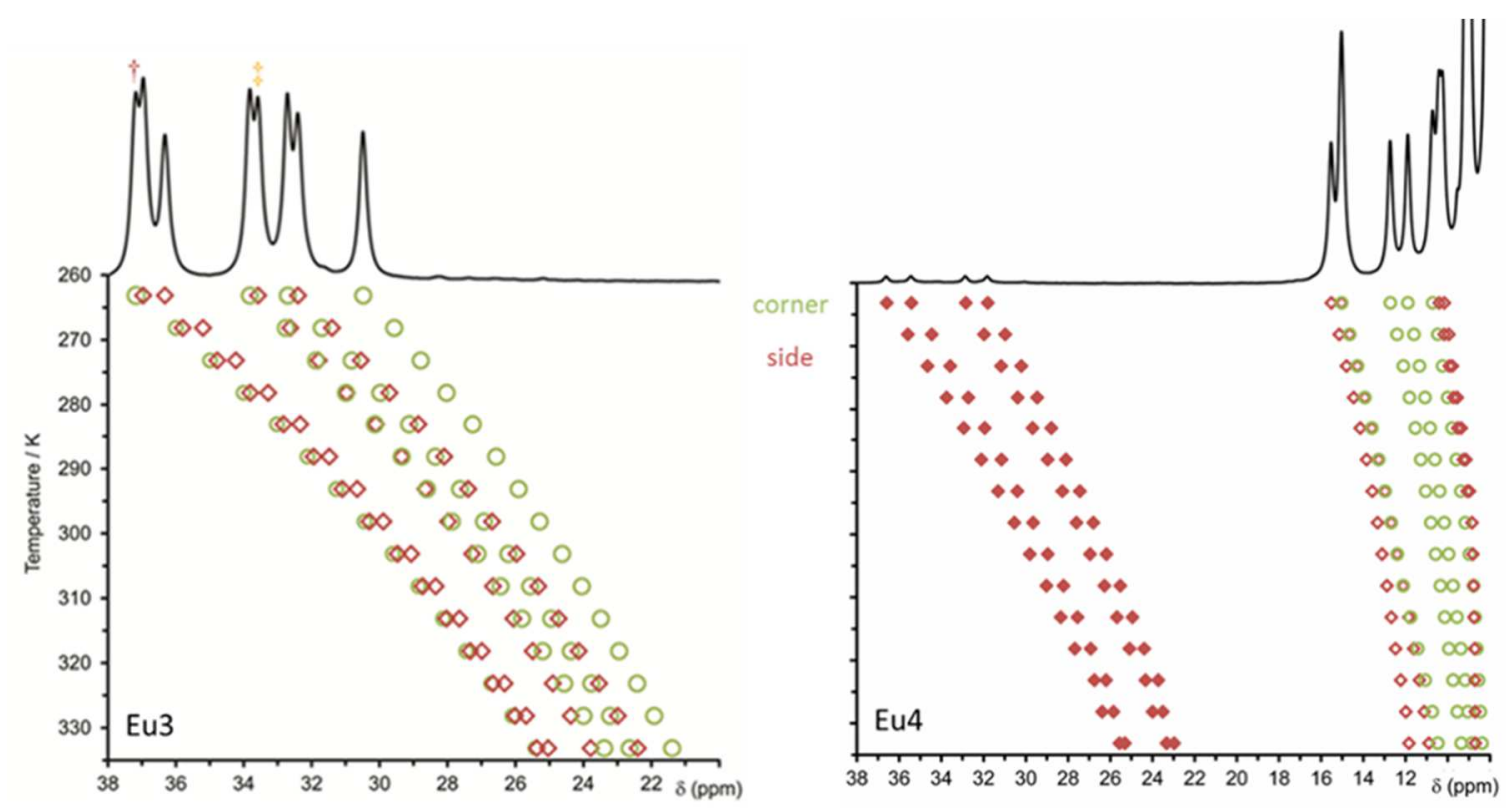

Figure 2.3.11. Eu3/4 variable temperature traces.

After identifying the shifting pattern of the side and corner regioisomers, determining which resonances in a spectrum belonged to the side and corner isomers became fairly obvious. Once regioisomers were identified, the areas under the resonances were measured and ratios of $\Lambda$-Ax and $\Delta$-Eq isomers could be determined for both the side and corner regioisomers for all of the Ln4 samples (Figure 2.3.12). When the ratios were plotted across the series, it was found that the transition from the $\Delta$-Eq isomer to the $\Lambda$-Ax isomer occurs 
earlier in the series for the side regioisomer than it does for the corner regioisomer. The side isomer begins to transition from TSAP to SAP isomer well before $\mathrm{Gd}^{3+}$, while the corner isomer doesn't appear to begin to transition until after $\mathrm{Gd}^{3+}$. This explains why only the " $s "-\Lambda$-Ax isomer was observed in the Eu4 sample and why the " $c$ "- $\Lambda$-Ax isomer was not observed.

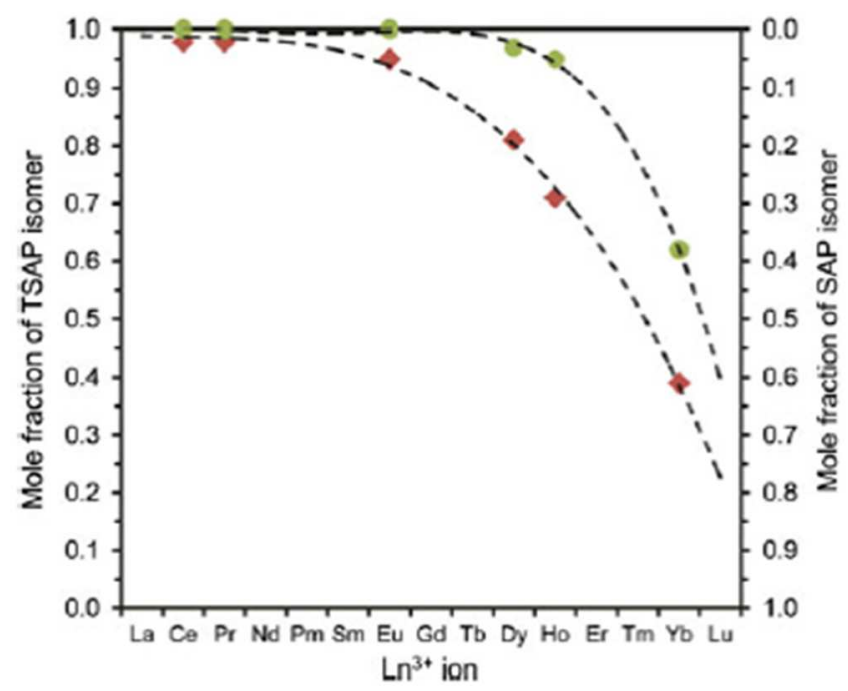

Figure 2.3.12. TSAP and SAP isomer ratio trends.

The trends indicate that the side isomer is better suited for smaller size lanthanide ions. The shift from TSAP isomer in early lanthanides to SAP isomer in the later lanthanides indicates that the SAP coordination cage is smaller than the TSAP isomer, and the trend for the side isomer to make that shift sooner in the lanthanide series indicates that the side coordination cage is likely smaller than the corner coordination cage. This would mean that the smallest coordination cage available to the lanthanide ions is the " $s$ "- $\Lambda$ isomer, the largest coordination cage available to the lanthanide ions is the " $c$ "- $\Delta$ isomer, and the "s"$\Delta$ and " $c$ "- $\Lambda$ isomer coordination cages are in between the larger and smaller coordination cages. 


\section{CEST Experiments}

CEST experiments were performed on several Ln3 and Ln4 isomers, including Pr3 and Pr4, Nd3, Eu3 and Eu4, and Yb4 (Figure 2.3.13). Some CEST effect from bound water was observed in all of the chelates run.
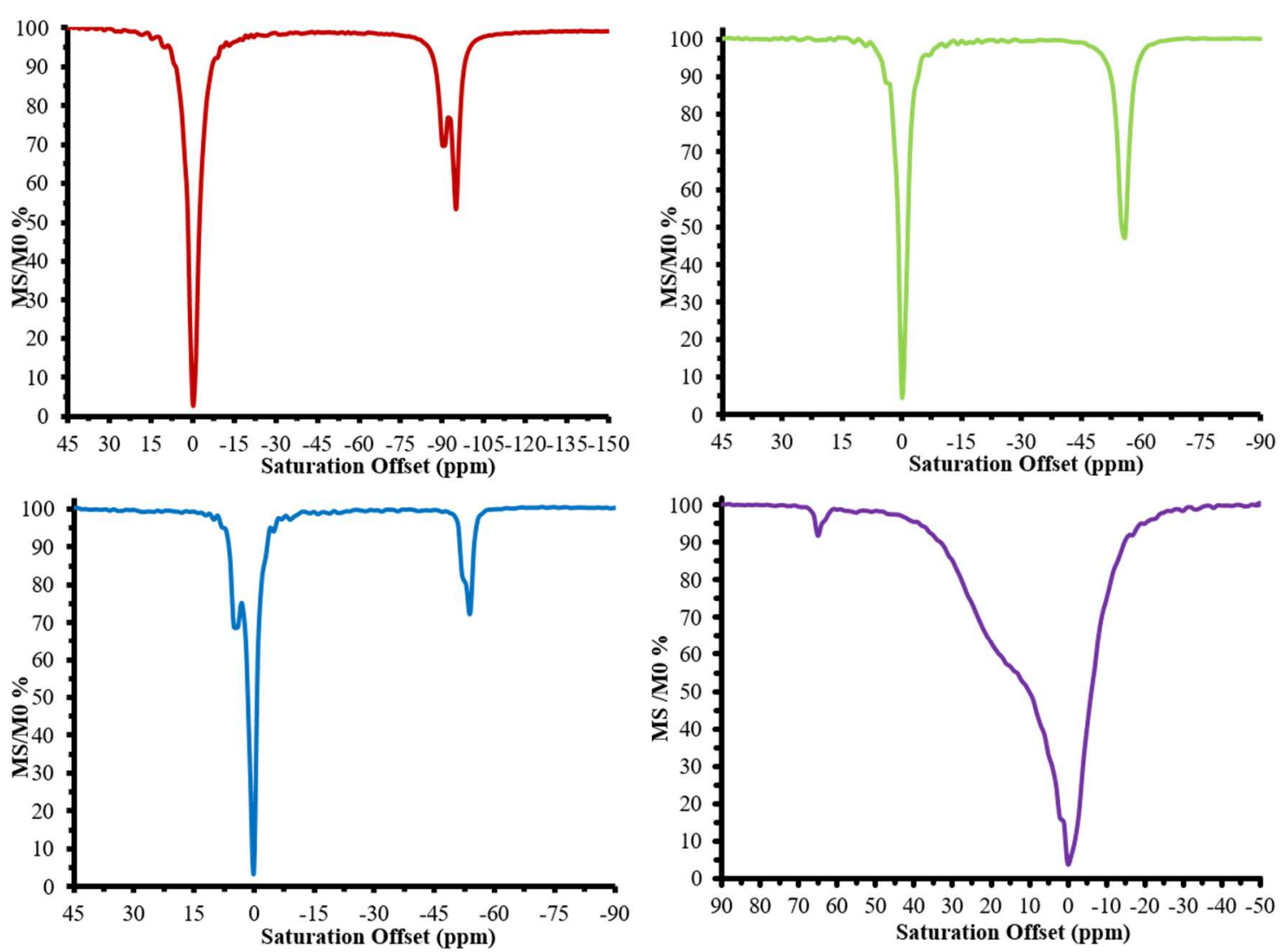

Figure 2.3.13. Ln3/4 CEST spectra. Top left: Pr3 (red). Top right: Pr4 (green). Bottom left: Nd3 (blue). Bottom right: $\mathrm{Yb} 4$ (purple).

However, only Eu3 and Eu4 were extensively studies so that comparisons could be made to the Eu1 and Eu2 CEST results that were previously obtained. 

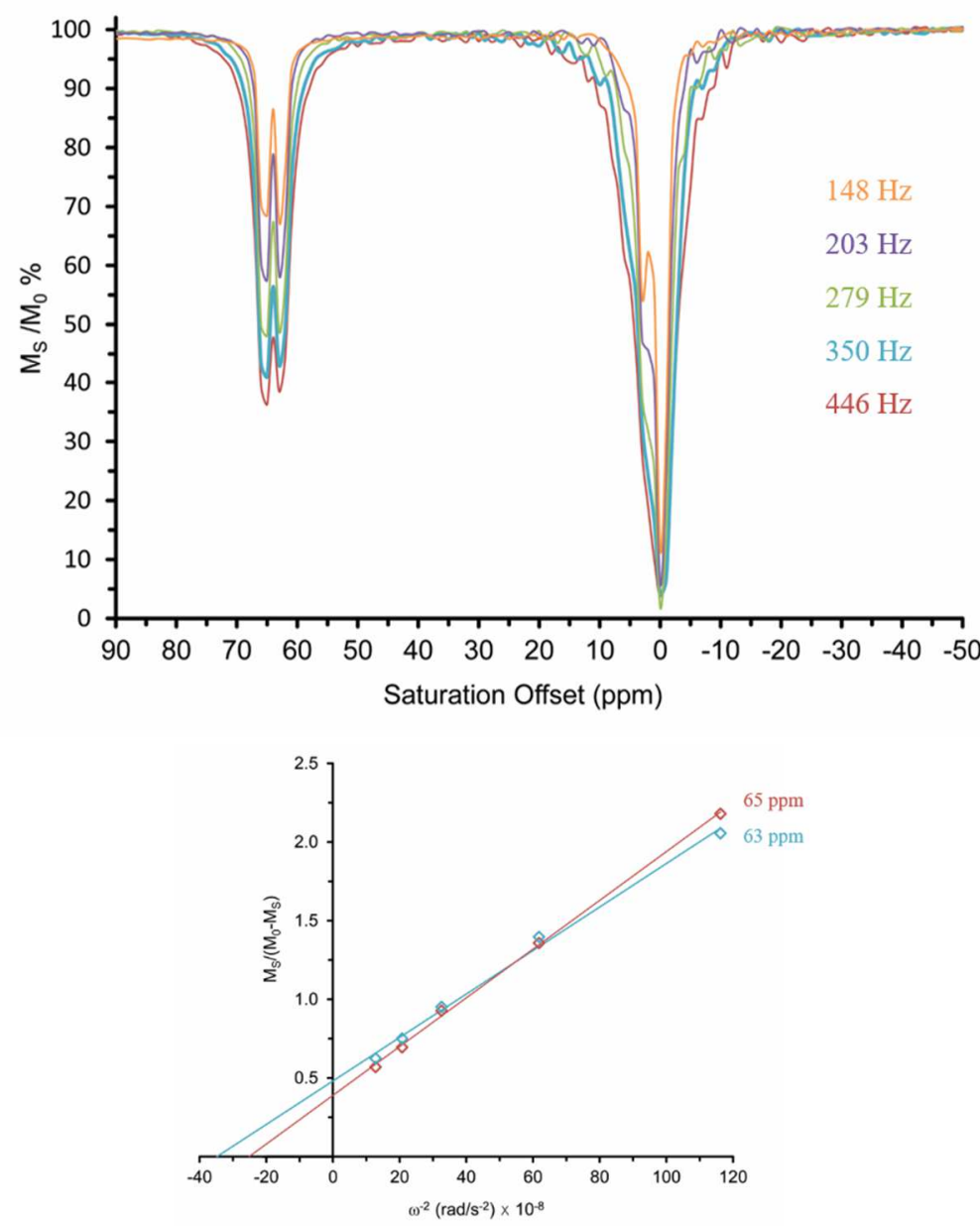

Figure 2.3.14. Eu3 CEST spectra and omega plot.

In the Eu3 sample, both the "s"- $\Lambda$-Eq isomer and " $c$ "- $\Lambda$-Eq isomer had CEST peaks where the bound water was expected to be (Figure 2.3.14). The intensities of the peaks increased with increasing $B_{1}$ power (increasing pulse power: orange, purple, green, blue, red), but the regioisomer that had the most intense peak changed as the power levels of the pre-saturation pulse changed. The water proton exchange rates of the two isomers were found to be very similar based first on the CEST peak widths and shapes and then confirmed second with omega plots generated from the varying $\mathrm{B}_{1}$ CEST data collected (Figure 2.3.14). The higher shifted peak represents the " $c$ "- $\Lambda$-Eq-Eu3 isomer with a water 
residence lifetime of $3.15 \mathrm{~ms}$ and the lower shifted peak represents the " $s$ " $\Lambda$-Eq-Eu3 isomer with a water residence lifetime of $3.70 \mathrm{~ms}$.

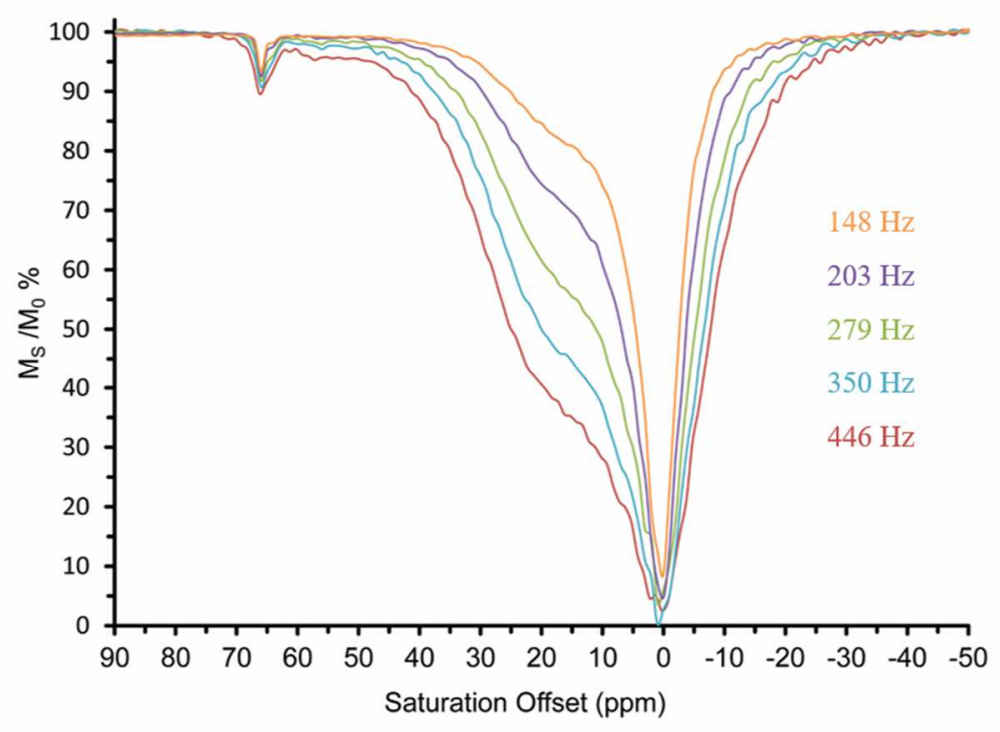

Figure 2.3.15. Eu4 CEST spectra.

The Eu4 data was more interesting with CEST peaks arising from the major TSAP isomers (" $s " / " c "-\Delta$-Eq-Eu4) and the minor SAP isomer ("s"- $s-A x-E u 4)$ present in solution (Figure 2.3.15). Due to their fast water exchange kinetics, TSAP isomers do not usually appear in CEST spectra. However, in the case of the " $s "-\Delta-E q-E u 4$ and " $c "-\Delta$-Eq-Eu4 isomers, the water exchange kinetics are slow enough for the isomers to appear in these spectra. The " $s "-\Lambda-A x-E u 4$ isomer present in solution had an observed CEST peak and the intensity of it increased as the pre-saturation pulse $\left(\mathrm{B}_{1}\right)$ power was increased (increasing pulse power: orange, purple, green, blue, red), which was expected. The " $s$ "- $\Lambda$-Ax-Eu4 isomer made up only approximately $2 \%$ of the total chelate in solution for the Eu4 sample, so the peak intensities were normalized with the respective chelate isomer concentrations to give $\% \mathrm{CEST} / \mathrm{mM}$ values, which allowed the " $s "-\Lambda$-Eq-Eu3 and " $c "-\Lambda$-Eq-Eu3 isomers and the " $s "-\Lambda-A x-E u 4$ isomer to be compared (Table 2.3.1). The percent CEST for the " $s$ "- 
$\Lambda$-Ax-Eu4 isomer was $47.1 \% \mathrm{CEST} / \mathrm{mM}$ and the percent CEST for the " $s$ "- $\Lambda$-Eq-Eu3 and " $c$ " $\Lambda$-Eq-Eu3 isomers was 10.4 and $8.6 \% \mathrm{CEST} / \mathrm{mM}$, respectively, at $148 \mathrm{~Hz}$, the lowest $\mathrm{B}_{1}$ power level used. The " $s$ "- $\Lambda$-Ax-Eu4 isomer had a greater percent CEST than either of the " $s " / " c "-\Lambda-E q-E u 3$ isomers.

Table 2.3.1. Eu3/4 SAP isomer \%CEST values and $\tau_{\mathrm{m}}$ values.

\begin{tabular}{cccccccc}
\hline \multicolumn{7}{c}{ CEST (\%/mM) } \\
$\begin{array}{c}\text { Peak } \\
(\mathrm{ppm})\end{array}$ & $\begin{array}{c}\mathrm{Eu} \\
(\mathrm{mM})\end{array}$ & $148 \mathrm{~Hz}$ & $203 \mathrm{~Hz}$ & $279 \mathrm{~Hz}$ & $350 \mathrm{~Hz}$ & $446 \mathrm{~Hz}$ & $\begin{array}{c}\boldsymbol{\tau}_{M}^{H} \\
(\mathrm{~ms})\end{array}$ \\
\hline 63 & 3.14 & 10.4 & 13.3 & 16.3 & 18.2 & 19.6 & 3.70 \\
\hline 65 & 3.64 & 8.6 & 11.7 & 14.3 & 16.2 & 17.5 & 3.15 \\
\hline 66 & 0.14 & 47.1 & 53.4 & 58.7 & 66.2 & 74.2 & $4.68-10.6$ \\
\hline
\end{tabular}

To get such a significant CEST signal from a minor isomer in solution means that the " $s$ "- $\Lambda$-Ax-Eu4 isomer is at least 4.5 times more efficient as a CEST agent than either of the " $s " /$ " $c$ "- $\Lambda$-Eq-Eu3 isomers at the lowest $B_{1}$ power level. This means that the " $s "-\Lambda-A x-$ Eu4 isomer has slower water exchange than the " $s " / “ c " c-\Lambda$-Eq-Eu3 isomers, indicating that the position of the amide substituent can affect the water exchange rate of the chelate, especially when the substituent has an electronic effect associated with it. 


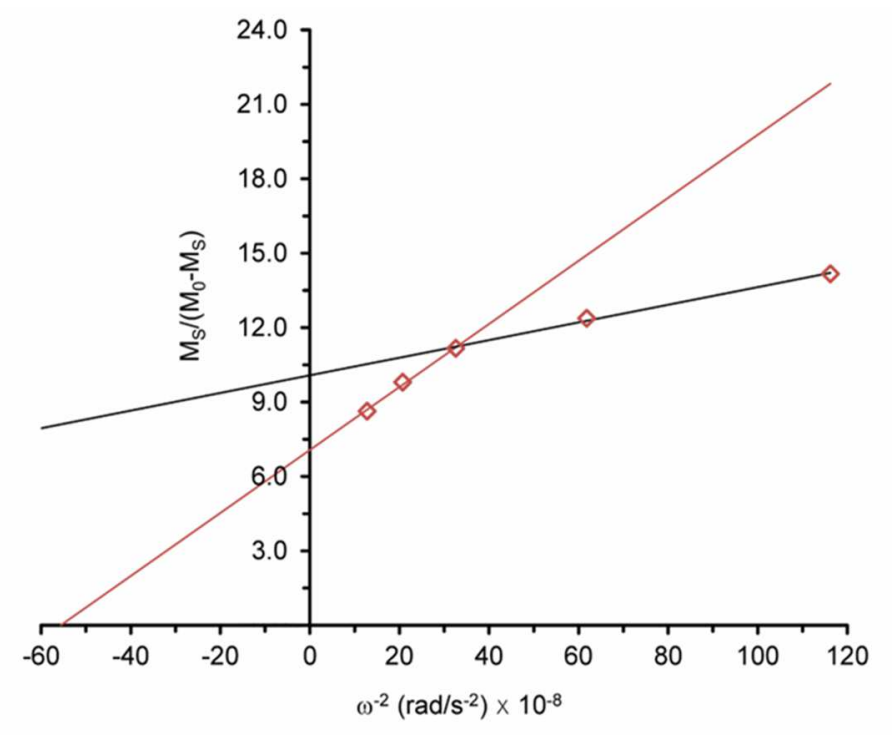

Figure 2.3.16. Eu4 omega plot.

When the omega plot for the "s"- $\Lambda$-Ax-Eu4 isomer was created (Figure 2.3.16), the line produced was non-linear and did not give a single value for the water residence lifetime. A wide range, 4.68 to $10.6 \mathrm{~ms}$, was found instead and it was much slower than the water residence lifetimes found for either of the " $s " / " c "-\Lambda$-Eq-Eu3 isomers. The nonlinear results of the omega plot were first thought to be an instrumental error. However, upon further inspection, it was noted that the CEST peaks for the " $s$ "- $\Lambda$-Ax-Eu4 isomer were slightly asymmetrical, suggesting a second SAP isomer present in solution that did not appear in any of the other NMR experiments performed. A second SAP isomer, presumably the " $c$ " $\Lambda$-Ax-Eu4 isomer, would explain the non-linearity of the omega plot and would be in exchange with the " $c$ "- $\Delta$-Eq-Eu4 isomer. The fact that the " $c$ "- $\Lambda$-Ax-Eu4 isomer might show up in the CEST experiment but no other NMR experiment suggests that it makes up less than the "s"- $\Lambda$-Ax-Eu4 isomer in solution. Despite its presumed low concentration, it has a very strong CEST effect indicating that it has extremely slow water exchange, slower than the "s"- $\Lambda$-Ax-Eu4 isomer. The limits of the water exchange rate 
range found from the two linear portions of the Eu4 omega plot could represent both isomers' water exchange rates, with the " $s$ "- $\Lambda$-Ax isomer having a water exchange rate of $4.68 \mathrm{~ms}$ and the "c"- $\Lambda$-Ax isomer having a water exchange rate of $10.6 \mathrm{~ms}$. The asymmetric nature of the Ln4 SAP isomer CEST peaks is also seen in the Yb4 CEST spectrum, where both the " $s$ "- $\Lambda$-Ax and " $c$ "- $\Lambda$-Ax isomers were observed in the ${ }^{1} \mathrm{H}$ NMRs taken (Figures 2.3.2 and 2.3.13). So the idea that the Eu4 sample contains both isomers is not completely unbelievable.

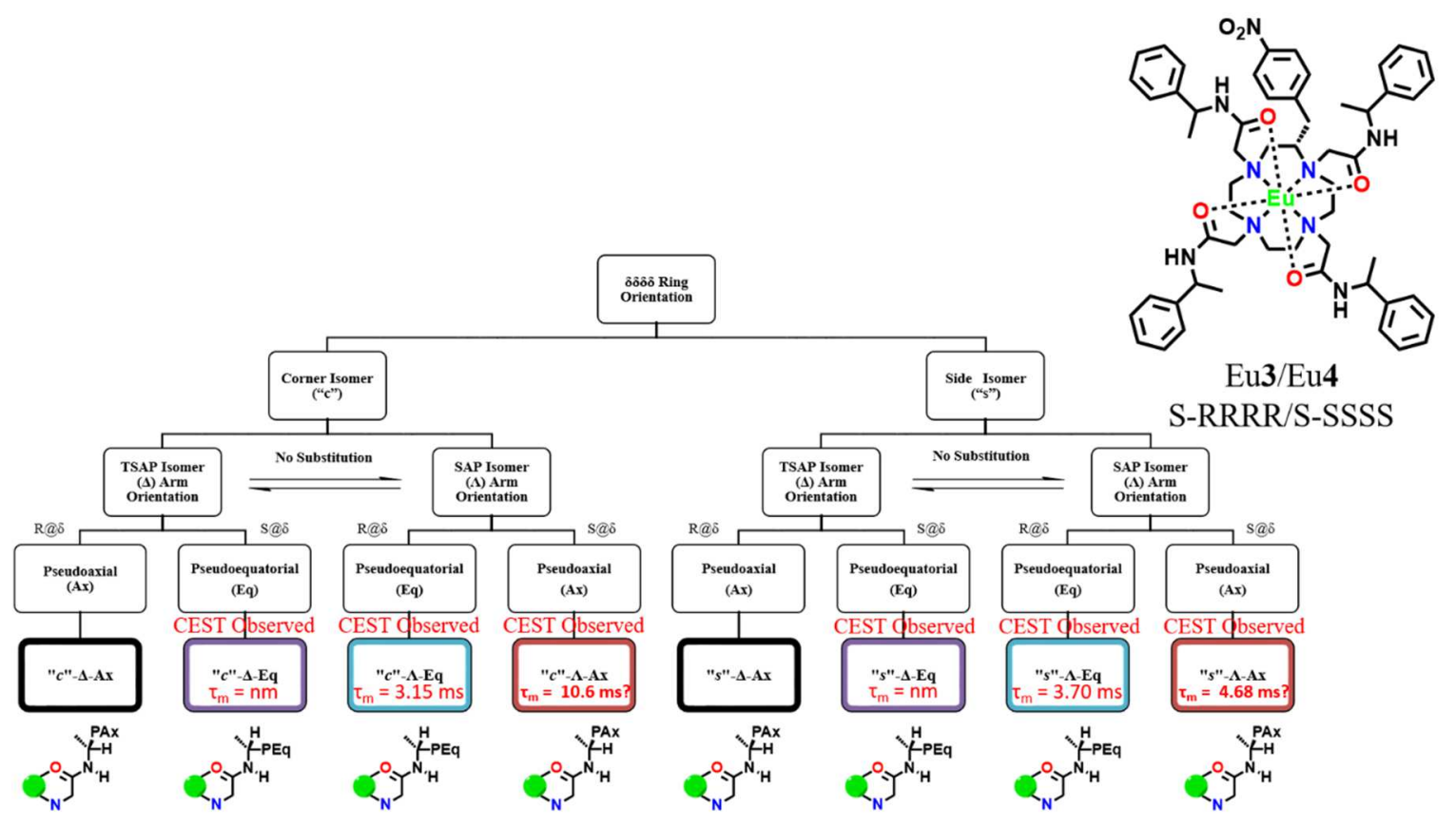

Figure 2.3.17. Eu3/4 CEST flowchart. Found $\tau_{\mathrm{m}}$ values are indicated. " $\tau_{\mathrm{m}}=\mathrm{nm}$ " means the $\tau_{\mathrm{m}}$ value was not measured.

These results suggest that the water proton exchange rates of the chelates are affected by the chirality of the $\delta$ substituted methyl group, mainly because of the nature of the substituent that is being moved in space. For the " $s " /$ " $c$ "- $\Lambda$-Eq-Eu3 isomers, the bulky hydrophobic group was in a pseudo-equatorial position (Figure 2.3.17) and therefore had little interaction with any water molecules attempting to access the water coordination site 
on the europium ion. However, with the " $s "-\Lambda-\mathrm{Ax}-\mathrm{Eu} 4$ isomer, and possibly the " $c$ "- $\Lambda$ Ax-Eu4 isomer, it is clear that there was a drastic slowing of the water exchange rate suggesting that the bulky hydrophobic phenyl group was interacting more with the water exchanging on and off of the chelate and could be explained by the group being in a pseudoaxial position (Figure 2.3.17) and creating a sort of hydrophobic tunnel that water has to travel through in order to coordinate to the metal.

\subsection{Conclusions}

Changing the amide substituent from a hydrophilic to a hydrophobic moiety enhanced the CEST effect seen in the $\mathrm{Eu}^{3+} \mathrm{NB}-\mathrm{DOTAM}$ chelates. The identification of an extremely effective CEST agent was complete, with the " $s$ "- $\Lambda$-Ax isomer having a \%CEST/mM over 4.5 times higher than the " $c$ "- $\Lambda$-Eq and " $s$ "- $\Lambda$-Eq isomers. The suggestion of the " $c$ "- $\Lambda$ Ax isomer being present in solution and being detectable in CEST experiments is also promising. Isolating each isomer and further studying the CEST effectiveness of each isomer would be the next logical step for this research, which is possible with the incorporation of an $\alpha$ position arm substitution added into the chelate structure and HPLC purification. 


\section{CHAPTER 3. ENCAPSULATION USING VESICLES}

\subsection{Vesicle Characterization}

\section{Vesicle Formation, Size and Stability}

Verifying the vesicle synthesis and characterizing the vesicles themselves was the first step of this project. Of particular interest was making sure the systems could be controlled experimentally and to know the volume of water associated with the inside and outside of the vesicles.

A range of vesicle sizes were made, $50 \mathrm{~nm}, 80 \mathrm{~nm}, 100 \mathrm{~nm}$, and $200 \mathrm{~nm}$, and the lipid compositions were $100 \%$ egg phosphatidylcholine (LAP, 60\%) and 1:1 egg phosphatidylcholine and cholesterol (LAP/C). The egg phosphatidylcholine lipid was 60\% pure with a variety of other lipids present, the exact composition of which was not attainable from the vendor. The vesicles were made by extruding a solution of water (10 $\mathrm{mL}$ ) and lipid at a concentration of $5 \mathrm{mg} / \mathrm{mL}$ through a polycarbonate membrane with pores of the desired sizes and dynamic light scattering (DLS) was performed to see the mean vesicle size of the sample. The sizes of the sample vesicles were monitored every day on the same instrument over a week to see how stable the vesicles were (Figure 3.1.1). Their mean sizes varied slightly over the week of monitoring, which may be an indication that the vesicles were above their phase transition temperature. Phosphatidylcholine has a phase transition temperature between $-5^{\circ} \mathrm{C}$ and $-15^{\circ} \mathrm{C}$, so the vesicles were definitely in the fluid phase at room temperature as opposed to being set into a solid gel phase below the phase transition temperature. The $50 \mathrm{~nm}$ sized vesicles had smaller variation in size over the week and the $200 \mathrm{~nm}$ sized vesicles had a larger variation over the week. The 50 and $80 \mathrm{~nm}$ 
extruded vesicles were larger than their intended sizes and the $200 \mathrm{~nm}$ extruded vesicles were smaller than their intended size, while the $100 \mathrm{~nm}$ extruded vesicles were their intended size. There was no trend in difference of size seen between the vesicles that contained cholesterol and the vesicles that did not contain cholesterol.

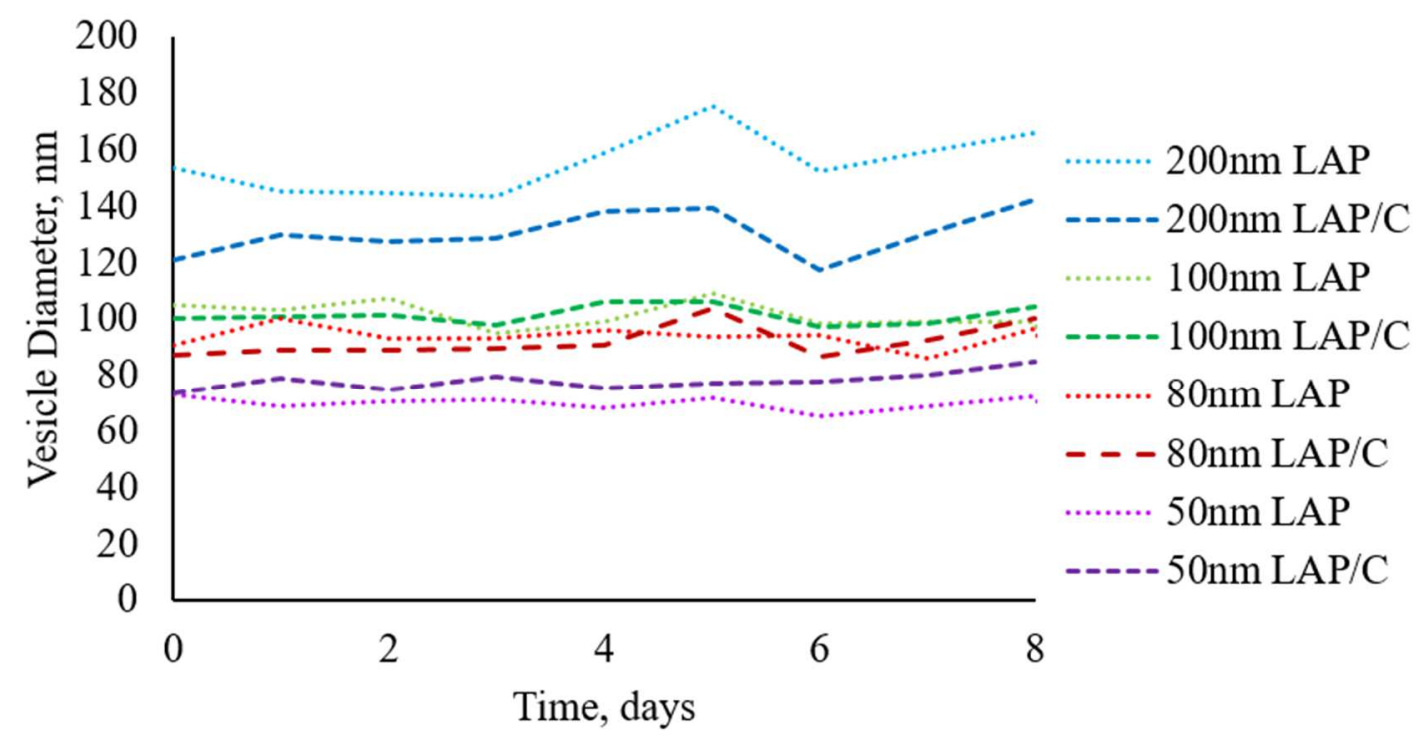

Figure 3.1.1. Vesicle stability over 8 days.

It was observed during vesicle synthesis that when cholesterol was added into the lipid mixture, the extrusion process through the polycarbonate membranes took significantly longer to perform and there was loss of lipid out of solution found on the membranes when the extrusion apparatus was taken apart for cleaning. Due to these observations, and the observations that the cholesterol seemed to offer no extra stability over time or control of vesicle size, it was decided that moving forward only lipid compositions consisting of the $100 \%$ egg phosphatidylcholine would be examined, without adding cholesterol.

\section{Complex Addition and Dialysis Control}

Once the stability of the vesicles had been gauged and control of the size of the vesicles was achieved, the next step was encapsulating complex inside the vesicles, removing 
excess chelate from the outside of the vesicles, and making sure that the vesicle size did not change from the dialysis process. Vesicles were made in buffer solutions containing varying amounts of $\mathrm{GdDTPA}^{-2}$, extruded to the desired sizes, and then dialyzed to remove any chelate from the outside of the vesicle while maintaining the desired size and shape of the vesicles. Dialysis was carried out with buffer baths being changed out at various time points to monitor whether or not the dialysis was working by looking at the $T_{1}$ values of the buffer baths. GdDTPA ${ }^{-2}$ is a $\mathrm{T}_{1}$ shortening agent. At the start of the dialysis process, the $T_{1}$ value of the buffer bath should be short because there should be more GdDTPA $^{-2}$ that is being pulled into the buffer bath. As the dialysis continues, the amount of GdDTPA $^{-2}$ being pulled into the buffer bath should decrease as the $\mathrm{GdDTPA}^{-2}$ is removed from outside of the vesicles and the $\mathrm{T}_{1}$ value should get longer as time goes on.

The first dialysis monitored was on a $5 \mathrm{mM} \mathrm{GdDTPA}^{-2} 100 \mathrm{~nm}$ vesicle sample and was performed at 8 hour intervals (Figure 3.1.2). The $\mathrm{T}_{1}$ values obtained for the baths indicated that the dialysis was essentially complete with all $\mathrm{GdDTPA}^{-2}$ removed from the outside of the vesicles by 24 hours. 


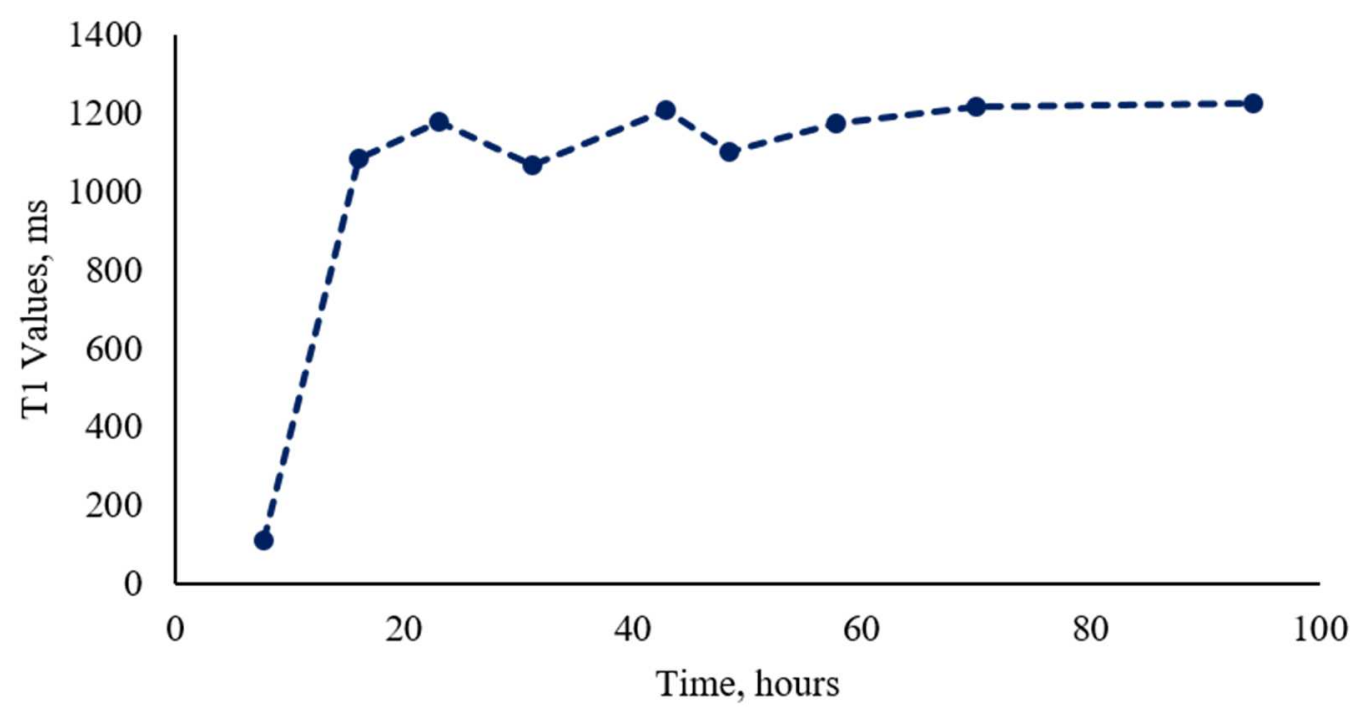

Figure 3.1.2. Dialysis bath $T_{1}$ values for $5 \mathrm{mM} \mathrm{GdDTPA}^{-2} 100 \mathrm{~nm}$ LaPC vesicles.

A second $5 \mathrm{mM} \mathrm{GdDTPA}^{-2} 100 \mathrm{~nm}$ vesicle synthesis and dialysis was performed, this time with the dialysis buffer bath being changed every hour for 8 hours and then changed one last time at 25 hours (Figure 3.1.3). The results showed the $T_{1}$ value was short at first and then it lengthened incrementally over the 8 hours that the baths were changed. The last buffer bath change at 25.8 hours showed a value similar to that obtained at 23 hours in the first dialysis bath monitoring experiment (1190 ms and $1178 \mathrm{~ms}$, respectively).

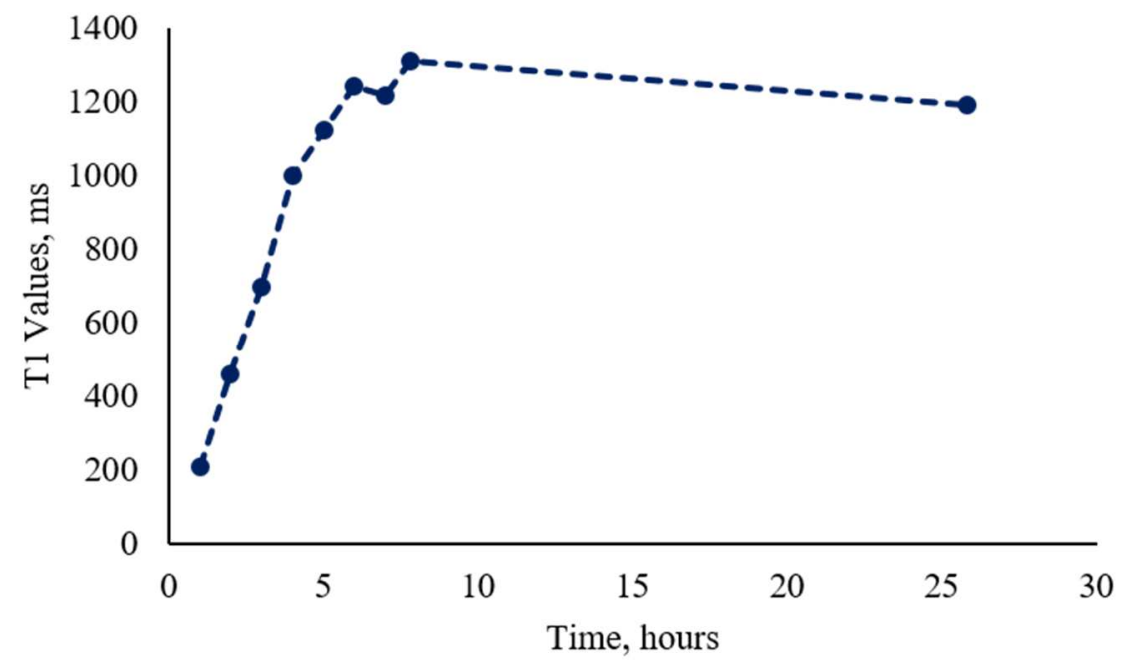

Figure 3.1.3. Dialysis bath $T_{1}$ values for $5 \mathrm{mM} \mathrm{GdDTPA}^{-2} 100 \mathrm{~nm}$ LaPC vesicles, second attempt. 
Before and after the dialysis was performed, the size of the vesicles was also monitored. If the osmolarity of the original buffer doesn't match the osmolarity of the dialysis bath, one of two things can happen: either the vesicles osmotically shrink or they osmotically swell. Both consequences are undesirable because they each compromise the shape and stability of the vesicles. Osmolarity is the concentration of a solution expressed as the total number of solute particles per liter. In the case of the buffers being used for this project, each buffer had $100 \mathrm{mOsm} \mathrm{NaCl}, 100$ mOsm hepes buffer, and whatever concentration of $\operatorname{GdDTPA}^{-2}$ was being tested $\left(20,15,5,4,3,2\right.$, or $\left.1 \mathrm{mM} \mathrm{GdDTPA}^{-2}\right)$. Osmotic shrinking occurs when the osmolarity of the solution on the inside of the vesicle is smaller than the osmolarity of the solution on the outside of the vesicle (Figure 3.1.4).
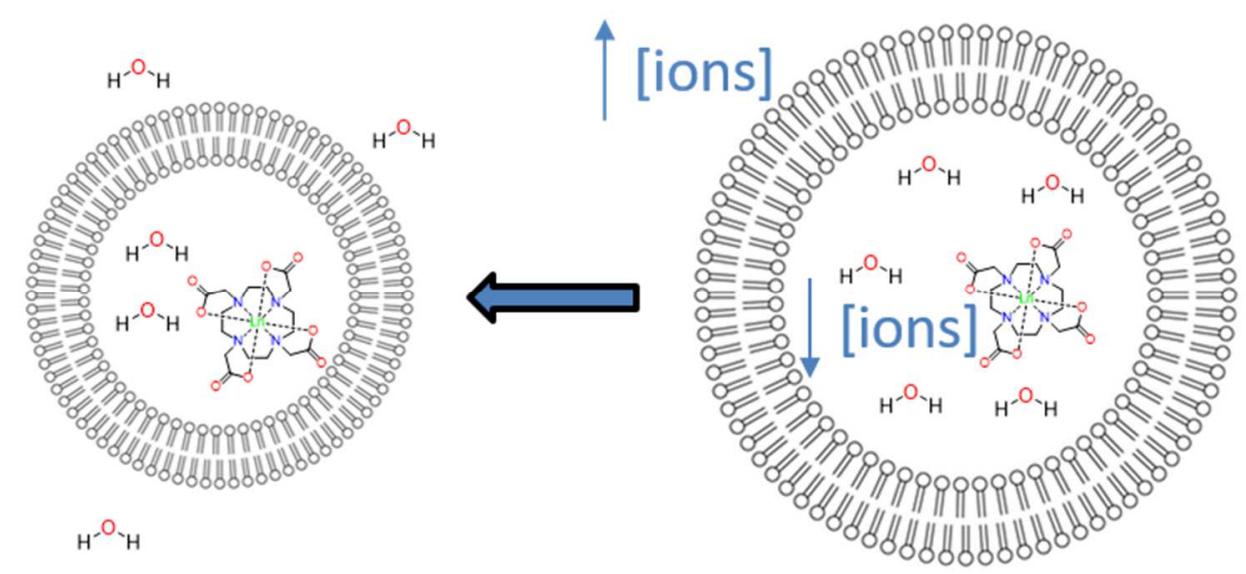

Figure 3.1.4. Osmotic shrinking mechanism.

The gradient of osmolarity that is created across the phospholipid bilayer by the different osmolarities causes water to move from the inside of the vesicle to the outside of the vesicle to equalize the osmolarities. Osmotic swelling occurs when the osmolarity of the solution on the outside of the vesicle is smaller than the osmolarity of the inside of the vesicle and water moves from the outside of the vesicle to the inside of the vesicle. 


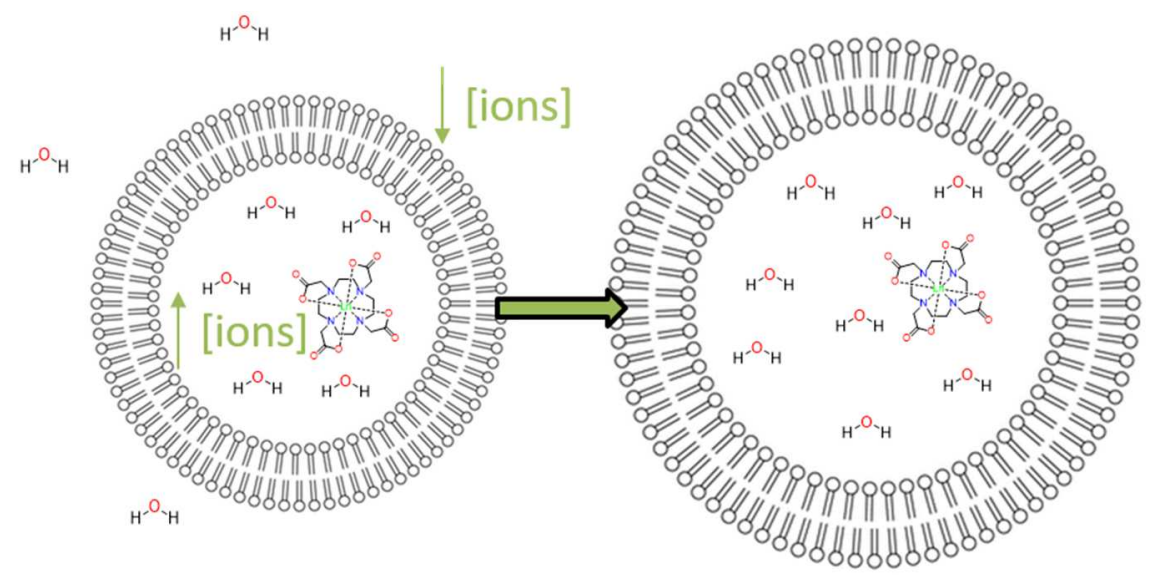

Figure 3.1.5. Osmotic swelling mechanism.

When vesicles shrink, the shape of the vesicle distorts from spherical to more of an egg shape. The distortion affects the water exchange efficacy of the vesicle and slows the water exchange rate because the lipids are stressed and pushed closer together as the curvature of the bilayer is changed. When vesicles swell, the phospholipid bilayer gets thinned out and stretched with the efficacy of the lipid packing being compromised. This leads to an increase in the water exchange kinetics across the lipid membrane.

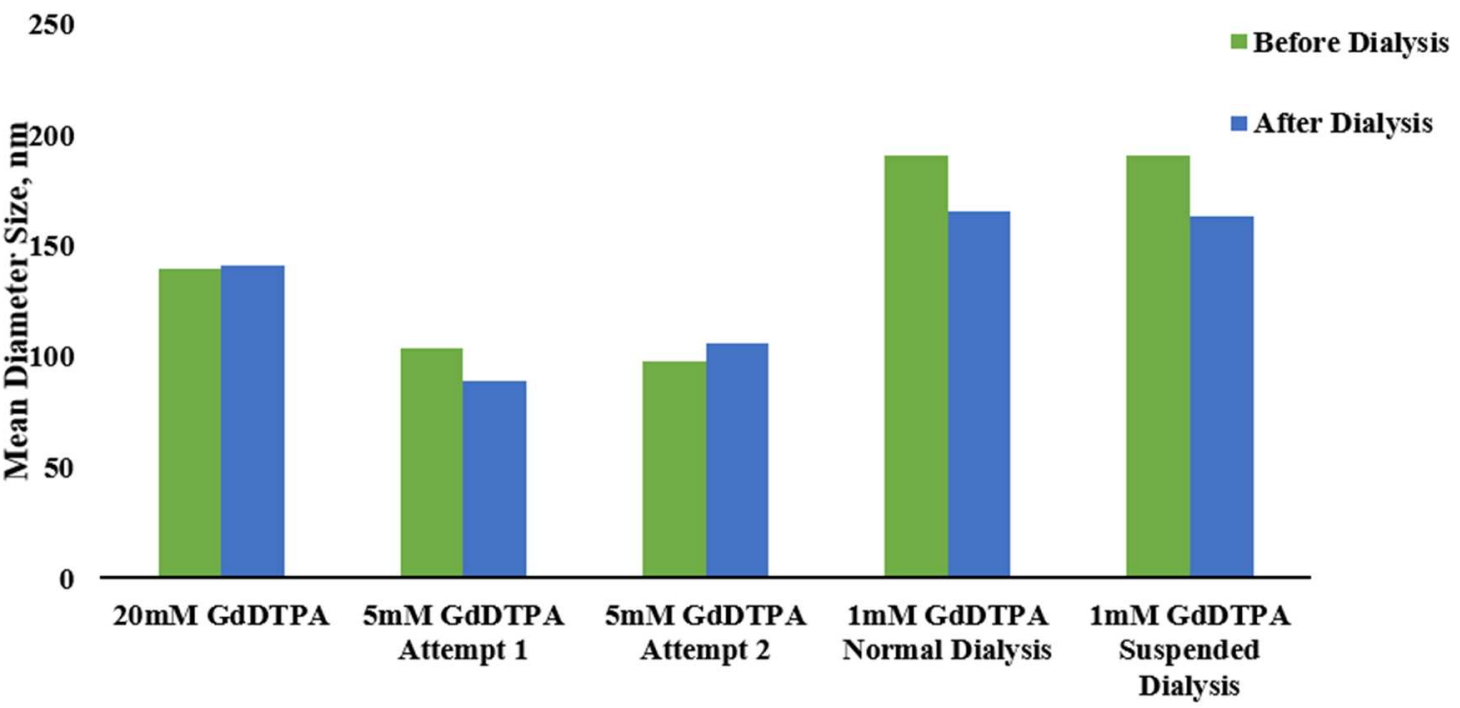

Figure 3.1.6. Mean vesicular diameter before and after dialization. 
Both of the aforementioned $5 \mathrm{mM} \mathrm{GdDTPA}^{-2} 100 \mathrm{~nm}$ samples shrank during dialysis. The change in size observed was considered enough to distort the shape of the vesicle, making it less spherical and more oval in shape. This distortion was expected to have an effect on the water exchange rate of the vesicles. In fact, several attempts were made to get the osmolality correct during dialysis so vesicles were not shrinking or swelling; however, despite redoing calculations, and making changes to the concentrations of the salts added to the buffer, all samples that were made and dialyzed shrank or swelled to some degree. The only sample that didn't shrink or swell was the $20 \mathrm{mM} \mathrm{GdDTPA}^{-2}$ samples (Figure 3.1.6). A suspended dialysis was also tried to see if concentrating the sample was possible, but it didn't afford any more concentrated of a sample and it also shrank the vesicles.

\subsection{T1 Analysis of Vesicle Systems}

$\mathrm{T}_{1}$ values are measured by using an inversion recovery experiment. In this experiment, the spins of the system are pulsed $180^{\circ}$ from the $\mathrm{z}$-axis to the $-\mathrm{z}$-axis, and the sample is allowed to return to the z-axis for some time, $\tau$. After time $\tau$, the spins are pulsed $90^{\circ}$ into the $\mathrm{x}-\mathrm{y}$ plane where the signal intensity is measured. This allows for a data point to be placed for that length of time $\tau$. The next time the spins are pulsed into the $-\mathrm{z}$-axis, the time $\tau$ is lengthened, and the spins are allowed to relax a little bit more to the z-axis, meaning that the intensity of the signal will be less than the previous $\tau$ time when the spins are pulsed into the $x-y$ plane. As some point, the time $\tau$ that the spins are allowed to return to equilibrium will allow the net sum of the spins to be in the z-axis before being pulsed into the $x-y$ plane. When this happens, the amount of signal read in the $x-y$ plane will begin to grow again until the spins are completely allowed to relax back to the z-axis with a longer 
time $\tau$. The amount of time that is required for the spins to completely relax back to the $\mathrm{z}-$ axis and the rate of return during time $\tau$ depends on the $T_{1}$ value of the sample (Figure 3.2.1). The VD (variable delay) list controls the values of $\tau$, and generally changes from sample to sample based on the $\mathrm{T}_{1}$ value. If the sample has a long $\mathrm{T}_{1}$ and a VD list for a short $\mathrm{T}_{1}$ is used with a short relaxation delay, scattering is possible since the sample doesn't have adequate time to relax back to equilibrium before the next inversion pulse is applied and the experiment will likely be cut off before the sample has time to fully relax with longer $\tau$ values.

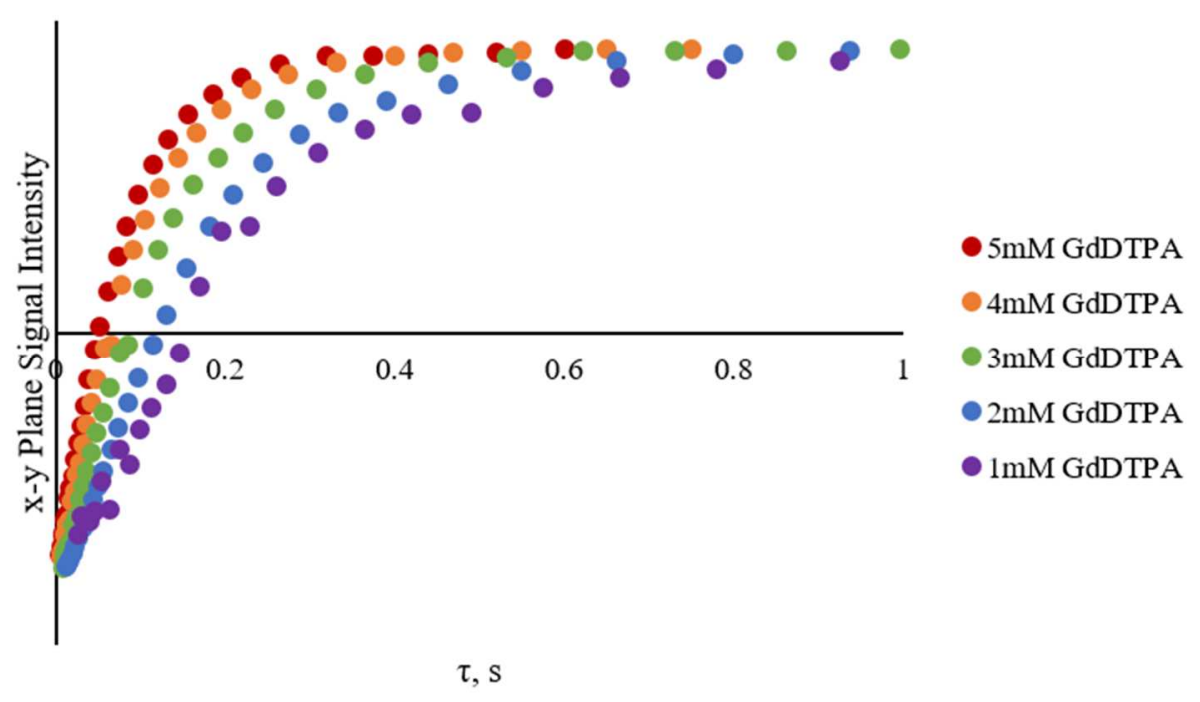

Figure 3.2.1. Various concentrated samples of $\mathrm{GdDTPA}^{-2}$ showing the changing rate of $\mathrm{T}_{1}$ relaxation.

\section{MHz T 1 Data}

Several attempts were made to probe the exchange kinetics across the phospholipid bilayer and to assess the ratio of internal and external water in the system using $\mathrm{T}_{1}$ recovery NMR experiments. Both the dialyzed and non-dialyzed samples were run at $20 \mathrm{MHz}$ and $25^{\circ} \mathrm{C}$ for $\mathrm{T}_{1}$ values. With the non-dialyzed samples, it was expected that one $\mathrm{T}_{1}$ value would be obtained (mono-exponential $\mathrm{T}_{1}$ systems) due to the fact that $\mathrm{GdDTPA}^{-2}$ was both inside 
and outside the vesicles and therefore the $T_{1}$ value on the inside and outside would be the same. The expected $T_{1}$ values for the non-dialyzed samples were calculated using the concentrations of GdDTPA ${ }^{-2}$ for each sample. The dialyzed samples were expected to have two $T_{1}$ values (bi-exponential $T_{1}$ systems), one for the inside of the vesicle where GdDTPA $^{-2}$ was still present and one for the outside of the vesicle where there was no longer any GdDTPA ${ }^{-2}$ after dialysis. The values of the two pools, the inside and the outside of the vesicles, depended on the exchange rate across the bilayer. If exchange was slow on the NMR timescale, the two compartments would have very different values; the inside of the vesicle would have a $T_{1}$ close to that of the undialyzed samples and the outside of the vesicles would have a $T_{1}$ value close to that of water. If exchange was fast on the NMR timescale, the two compartments would be considered well mixed and the values for the inside and outside of the vesicles would be close to the same. If exchange was somewhere in between slow and fast, then the two $T_{1}$ values found would have some other $T_{1}$ values representative of some mixing, not all or none.

When running samples on the $20 \mathrm{MHz}$ minispec at OHSU, the instrument fits the data and gives a $\mathrm{T}_{1}$ value for each sample. It can be controlled whether or not the program tries to fit the data to a monoexponential or a biexponential fitting, and the delay values used for the inversion recovery experiment can be changed. However, there is not actual manipulation of the data done by the researcher. Everything is done on the instrument by the program.

$T_{1}$ values were not realistic, with one always far too long or too short to be a real $T_{1}$. One $T_{1}$ value was expected to be obtained for the non-dialyzed samples and two $T_{1}$ values 
for the dialyzed samples. What was observed was that as the concentration of the GdDTPA $^{-2}$ decreased inside the vesicles, the $T_{1}$ value obtained for the non-dialyzed samples lengthened. Also observed was the fact that each sample had the $\mathrm{T}_{1}$ values go from short to long in the undialyzed to dialyzed samples, respectively. This data shows that the dialyzation to remove external $\mathrm{GdDTPA}^{-2}$ was successful (Figure 3.2.2). However, the dialyzed samples had no clear trend.

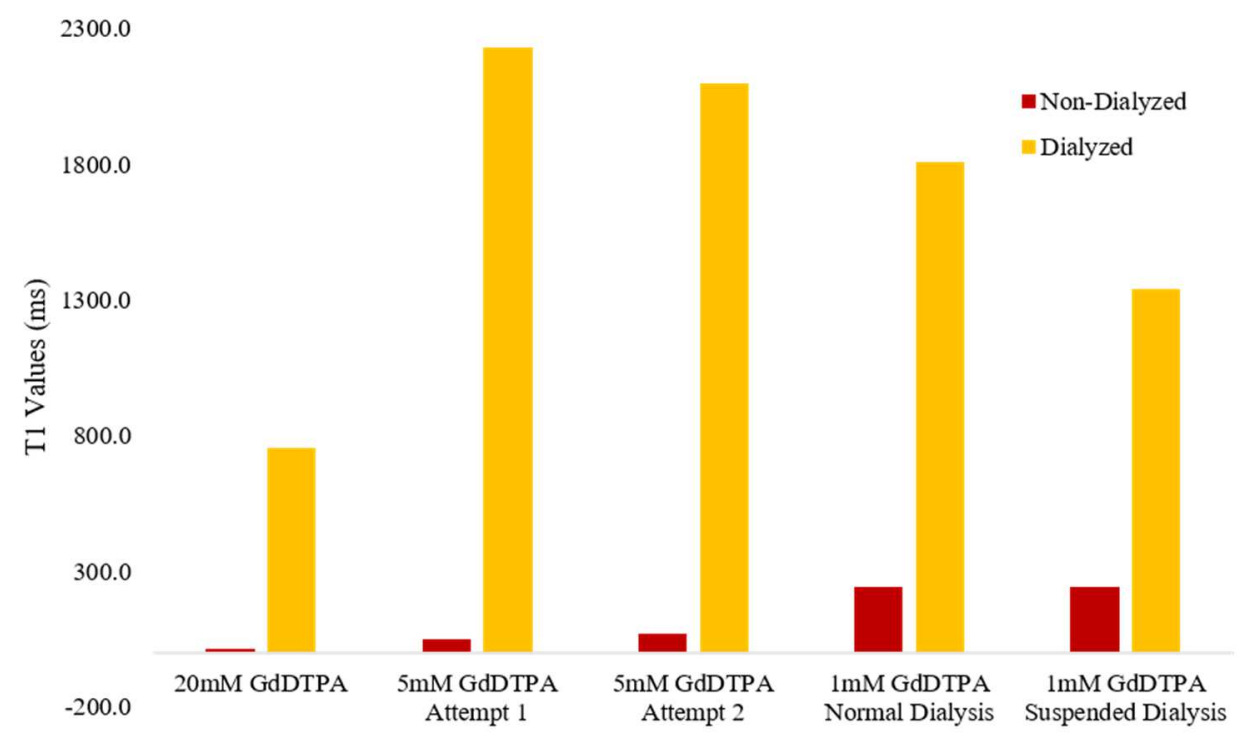

Figure 3.2.2. $T_{1}$ values of vesicle containing GdDTPA $^{-2}$ samples before and after dialysis.

Water's expected $T_{1}$ value is at least a couple of seconds. The exact value is unknown, and attempting to run just water on the spectrometer proved to be futile. The $20 \mathrm{mM}$ $\mathrm{GdDTPA}^{-2}$ vesicles appeared to have very rapid exchange of water across the phospholipid bilayer of the vesicles because the $T_{1}$ value of the dialyzed sample is very short compared to what would be expected for water. This means that water from the inside of the vesicle, which has a shortened $\mathrm{T}_{1}$ value due to the GdDTPA ${ }^{-2}$ being present, was leaving the vesicle and exchanging with the bulk water outside of the vesicle quickly enough to have an observed shortening of the $T_{1}$ value of the outside of the vesicle. As the concentration of 
GdDTPA $^{-2}$ was decreased inside the vesicles, the shortening of the outside water should become less drastic, with the 5 and $1 \mathrm{mM} \mathrm{GdDTPA}^{-2}$ vesicles having longer $\mathrm{T}_{1}$ values for the dialyzed samples than the $20 \mathrm{mM} \mathrm{GdDTPA}^{-2}$ vesicles. However, with the $5 \mathrm{mM}$ and 1 $\mathrm{mM} \mathrm{GdDTPA}^{-2}$ vesicles, the expected lengthening of $\mathrm{T}_{1}$ values was the opposite of what was observed. The $\mathrm{T}_{1}$ values for the $1 \mathrm{mM}$ vesicles were shorter, not longer.

There were a couple of explanations for this. One was that the $5 \mathrm{mM} \mathrm{GdDTPA}^{-2}$ vesicles were $100 \mathrm{~nm}$ vesicles and the $1 \mathrm{mM} \mathrm{GdDTPA}^{-2}$ vesicles were $200 \mathrm{~nm}$ vesicles. With different sizes of vesicles, it is hypothesized that vesicles will appear to have different water exchange kinetics. Since the vesicles were different sizes, comparing them directly could not actually be done. Keeping one of the parameters (either concentration of chelate or size of vesicle) constant could have helped to explain the trend that was seen. Another explanation as to why the $T_{1}$ values were reversed from what was expected was that the lipid concentration used when the vesicle batches were made were different. The $5 \mathrm{mM}$ GdDTPA $^{-2}$ vesicles were synthesized with a lipid concentration of $5 \mathrm{mg} / \mathrm{mL}$. The $1 \mathrm{mM}$ GdDTPA $^{-2}$ vesicles were synthesized with a lipid concentration of $20 \mathrm{mg} / \mathrm{mL}$. The change in lipid concentration when the vesicles were made could have affected the permeability of the vesicles. However, it would make sense that a more concentrated lipid bilayer would be less permeable to water and therefore would have a longer $T_{1}$ value for the dialyzed sample. This is again opposite of what was observed.

Another problem noted with the data collected on the $20 \mathrm{MHz}$ spectrometer was that the values found for one sample could change from one run to another. So if one sample was run two times in a row, the resulting data could differ drastically. In one run, the sample 
being run would be monoexponential and in a second run the sample would be biexponential. If the monoexponential fit was turned on, generally one $\mathrm{T}_{1}$ value was given. However, if the biexponential fit was turned on, two $T_{1}$ values were not always given. And if a sample was supposed to be monoexponential, like undialyzed samples, the program would give two $T_{1}$ values as if it was biexponential. Sometimes $T_{1}$ values would differ by hundreds of ms. Since the results that were obtained were not reproducible on the $20 \mathrm{MHz}$ instrument, data collection was moved to the $600 \mathrm{MHz}$ instrument on PSU's campus.

\section{$600 \mathrm{MHz} \mathrm{T}_{1}$ Data}

When data collection was moved to the $600 \mathrm{MHz}$, another change to sample prep was made. Since keeping vesicles from shrinking and swelling after dialysis was proving to be difficult, it was suggested that the chelate being used could be put on the outside of the vesicle instead of the inside of the vesicle. This alleviated the need to dialyze the samples and presumably would keep the vesicles from shrinking or swelling. For these experiments, the samples were made using a buffer with only $100 \mathrm{mOsm} \mathrm{NaCl}$ and $100 \mathrm{mOsm}$ hepes buffer and no complex. Once the vesicles had been extruded, then complex was added to the samples. This method allowed for several different samples to be made at once instead of one sample being made each time the extruder was used. For these samples, 10 (or at least 5) $\mathrm{mL}$ samples were extruded, ensuring that each different concentration sample contained the same concentration of lipid and were the same sizes. The large sample of formed vesicles were split into $1 \mathrm{~mL}$ samples and the desired amounts of GdDTPA ${ }^{-2}$ were added to each sample in the smallest amount of stock buffer to make a range of concentrated samples $\left(15,5,4,3,2\right.$, or $\left.1 \mathrm{mM} \mathrm{GdDTPA}^{-2}\right)$. The stock buffer contained $1 \mathrm{M}$ 
GdDTPA $^{-2}, 100$ mOsm NaCl, and 100 mOsm hepes buffer. The amounts added to the solutions were on the order of $\mu \mathrm{L}$.

The experiments on the $600 \mathrm{MHz}$ make up the majority of the T1 experiments run. While the LAP vesicles were primarily studied, some parameters were changed (see table). Lipid composition was changed to incorporate cholesterol and also changed completely to DPPC, concentration of the lipid used was changed from $5 \mathrm{mg} / \mathrm{mL}$ to $25 \mathrm{mg} / \mathrm{mL}$, the vesicle samples were concentrated down after formation using centrifugation, and the formation of the vesicles was also altered to include freeze-thawing (FT) cycles before the extrusion process. The VD list used in the collection of the data was also changed. Generally, a concentration specific VD list was used, but including a concentration independent VD list was used in hopes that the longer T1 value would be better represented in the data collected. Collecting data on the $600 \mathrm{MHz}$ instrument required that the data be worked up manually and be fitted manually. Two different methodologies each were used to analyze the data and fit the data.

\section{Processing and Fitting the $600 \mathrm{MHz}$ Data}

The original $(\mathrm{O})$ processing method involved analyzing the data using the $\mathrm{T}_{1}$ analysis method on the Bruker program. In this method, an integration was made to the phased last spectrum taken (the one with the most signal) to indicate to the program what peak to look at to pick data points. Then the program pulls the data (tau value, ppm associated with the peak measured, integral of that peak, and the intensity value of that peak) and creates a recovery curve of data, which was then exported into a text file and imported into an excel sheet. From there, the data was manipulated in excel. 
All of the parameter changes are presented in Table 3.2.1. In the following graphs, the original (O) method is represented by blue bars and the professional (P) method is represented by green bars. Solid bars are representative of monoexponential (M) fitting results and open bars are representative of biexponential (B) fitting results. Bars filled with lines represent $T_{1}$ values that had population size values of 0 . Black dashed lines are representative of a calculated $T_{1}$ value for water, which was found from graphing the concentration of free GdDTPA ${ }^{-2}$ solutions versus the relaxivity of each solution and red dotted lines represent the $T_{1}$ values found from those concentrations of GdDTPA ${ }^{-2}$ solutions. Purple dotted lines, which are only found on $15 \mathrm{mM} \mathrm{GdDTPA}^{-2}$ graphs, are a calculated value for the expected $T_{1}$ value. The dashed and dotted lines are included in the graphs to help give ideas of where expected values might have been. 
Table 3.2.1. Changing parameters for comparisons and better data fitting.

\begin{tabular}{|c|c|c|c|c|}
\hline $\begin{array}{l}\text { Parameter } \\
\text { Changing }\end{array}$ & Base & Change Made & $\begin{array}{l}\text { Expected Change in Water } \\
\text { Exchange Rate }\end{array}$ & Expected Change on $T_{1}$ Values \\
\hline $\begin{array}{c}\text { Lipid } \\
\text { Composition }\end{array}$ & LaPC Only & $\begin{array}{l}\text { add cholesterol } \\
\text { use DPPC } \\
\text { use DPPC + add } \\
\text { cholesterol } \\
\end{array}$ & $\begin{array}{l}\text { slow water exchange across } \\
\text { membrane }\end{array}$ & $\begin{array}{l}2 \text { distinct species: } 1 \mathrm{~T}_{1} \text { value consistent } \\
\text { with the GdDTPA concentration and } 1 \mathrm{~T}_{1} \text { value } \\
\text { consistent with water }\end{array}$ \\
\hline $\begin{array}{l}\text { Starting Lipid } \\
\text { Composition }\end{array}$ & $5 \mathrm{mg} / \mathrm{mL}$ & $\begin{array}{l}\text { increase to } 25 \\
\mathrm{mg} / \mathrm{mL}\end{array}$ & $\begin{array}{l}\text { slow water exchange across } \\
\text { membrane }\end{array}$ & $\begin{array}{l}2 \text { distinct species: } 1 \mathrm{~T}_{1} \text { value consistent } \\
\text { with the GdDTPA concentration and } 1 \mathrm{~T}_{1} \text { value } \\
\text { consistent with water }\end{array}$ \\
\hline $\begin{array}{c}\text { Method of } \\
\text { Vesicle Formation }\end{array}$ & $\begin{array}{c}\text { Film } \\
\text { Hydration and } \\
\text { Extrusion } \\
\end{array}$ & $\begin{array}{l}\text { Film Hydration, } \\
\text { Repeated Freeze-Thaw } \\
\text { Cycles, and Extrusion } \\
\end{array}$ & $\begin{array}{l}\text { should not change water } \\
\text { exchange rates; should ensure that } \\
\text { the system is only } 2 \text { site exchange }\end{array}$ & more reliable biexponential fitting result \\
\hline Vesicle Size & $200 \mathrm{~nm}$ & $\begin{array}{l}50 \mathrm{~nm}, 80 \mathrm{~nm}, \\
100 \mathrm{~nm}, 1 \mu \mathrm{m}\end{array}$ & $\begin{array}{l}\text { increase exchange rate in the } \\
\text { smaller vesicles; decrease } \\
\text { exchange rate in the larger vesicles }\end{array}$ & $\begin{array}{l}\text { larger vesicles exhibiting } 2 \text { distinct species; } \\
\text { smaller vesicles not exhibiting } 2 \text { distinct }\end{array}$ \\
\hline $\begin{array}{l}\text { Concentration } \\
\text { of Vesicles }\end{array}$ & $5 \mathrm{mg} / \mathrm{mL}$ & $\begin{array}{l}\text { increase to } 24,33 \text {, } \\
\text { and } 215 \mathrm{mg} / \mathrm{mL}\end{array}$ & $\begin{array}{c}\text { should not change water } \\
\text { exchange rates; should make the } \\
\text { volume ratio } \mathrm{H}_{2} \mathrm{O}_{\mathrm{i}}: \mathrm{H}_{2} \mathrm{O}_{\mathrm{e}} \text { more } \\
\text { equal } \\
\end{array}$ & $\begin{array}{l}\text { more reliable biexponential fitting result } \\
\text { with equal contributions to the } T_{1} \text { values }\end{array}$ \\
\hline $\begin{array}{c}\text { Data } \\
\text { Collection Method }\end{array}$ & $\begin{array}{l}\text { Concentration } \\
\text { Dependent } \\
\text { VDList } \\
\end{array}$ & $\begin{array}{c}\text { Concentration } \\
\text { Independent VDList }\end{array}$ & $\begin{array}{l}\text { should not change water } \\
\text { exchange rates }\end{array}$ & $\begin{array}{l}\text { accurate } T_{1} \text { values should be obtained since } \\
\text { all of the signal (primarily that of the species } \\
\text { with the longer } T_{1} \text { ) is collected }\end{array}$ \\
\hline $\begin{array}{l}\text { Data Analysis } \\
\text { Method }\end{array}$ & $\begin{array}{l}\text { Original }(\mathrm{O}) \\
\text { Method }\end{array}$ & $\begin{array}{l}\text { Professional (P) } \\
\text { Method }\end{array}$ & $\begin{array}{l}\text { should not change water } \\
\text { exchange rates; should make fitting } \\
\text { data easier and should produce } \\
\text { more accurate results }\end{array}$ & $\begin{array}{l}\text { accurate } T_{1} \text { values should be obtained since } \\
\text { the peaks used in analysis are better phased and } \\
\text { integral instead of peak intensity is used }\end{array}$ \\
\hline
\end{tabular}


CHAPTER 3. ENCAPSULATION

\section{Lipid Composition Comparison}

The first study that was performed on the $600 \mathrm{MHz}$ was to look at how the composition of the lipid changed the exchange rates. Initially, only phosphatidylcholine (LaPC) was used in the preparation of the vesicles. Cholesterol was eventually added in a 1:1 molar ratio $(\mathrm{LaPC}+\mathrm{C})$. The beginning concentration of the lipid was either $5 \mathrm{mg} / \mathrm{mL}$ or 25 $\mathrm{mg} / \mathrm{mL}$, and the vesicles were extruded at $200 \mathrm{~nm}$ or not at all (Non Extruded).

All of the samples (Figure 3.2.3) gave monoexponential (M) fittings with $\mathrm{T}_{1}$ values around the free $5 \mathrm{mM} \mathrm{GdDTPA}^{-2} \mathrm{~T}_{1}$ value for both the original $(\mathrm{O})$ and the professional (P) analysis method. In the original analysis method, the biexponential (B) fittings, except for the Non Extruded $\mathrm{LaPC}$ at $25.06 \mathrm{mg} / \mathrm{mL}$ initial lipid concentration, gave one $\mathrm{T}_{1}$ value near the free $5 \mathrm{mM} \mathrm{GdDTPA}^{-2} \mathrm{~T}_{1}$ value and one $\mathrm{T}_{1}$ value that was above $200 \mathrm{~s}$. The $\mathrm{T}_{1}$ values that were above $200 \mathrm{~s}$ all had population sizes of 0 associated with them. This means that the value is erroneous and not present in the sample; it is simply just a value that the fitting put in as a place holder. In the professional analysis method, the biexponential fittings all gave two $T_{1}$ values close to the free $\mathrm{GdDTPA}^{-2} \mathrm{~T}_{1}$ value and the population sizes were between $80-70 \%$ and $20-30 \%$. No real improvement was made by adding cholesterol, increasing the amount of starting lipid, or even by extruding the samples. These data suggest that the exchange rate across the phospholipid bilayer is fast on the NMR timescale and that the two pools of water in the system (the external with $\operatorname{GdDTPA}^{-2}$ and the internal without GdDTPA ${ }^{-2}$ ) are well mixed. 


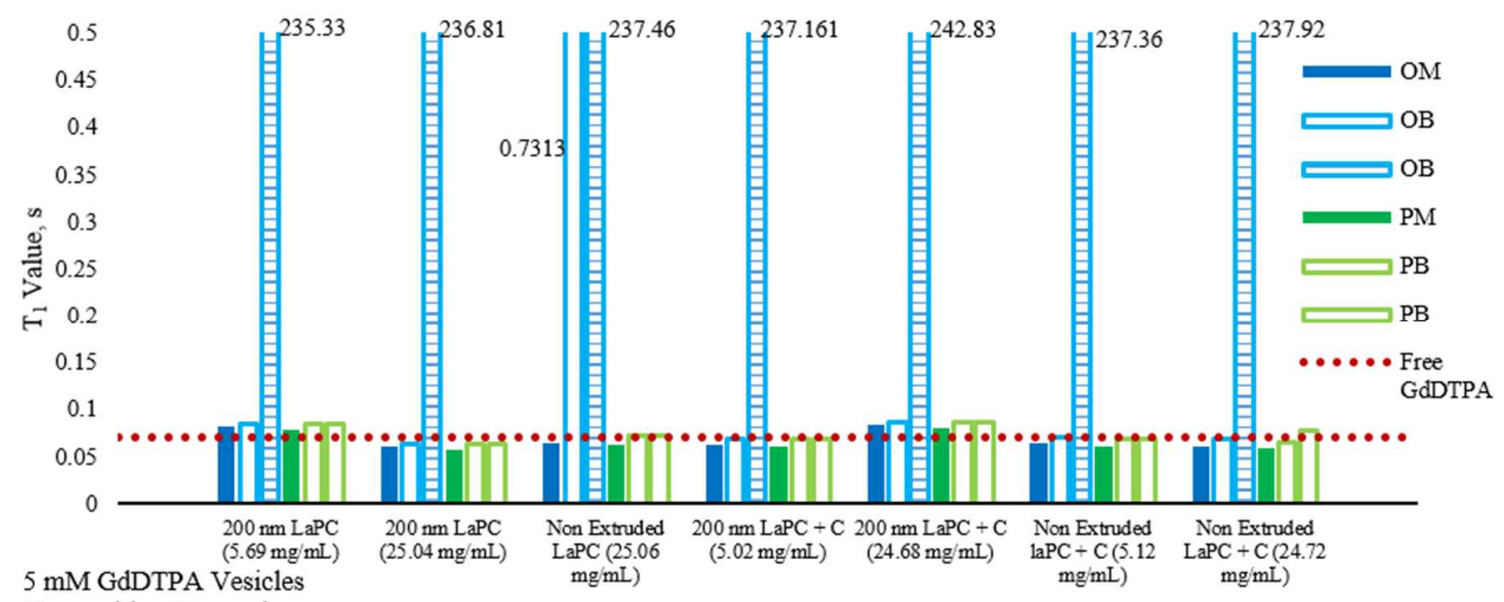

Composition Comparison

Figure 3.2.3. $5 \mathrm{mM} \mathrm{GdDTPA}^{-2}$ vesicle systems.

DPPC was also looked into as an alternative lipid, however, proper formation of the vesicles proved to be difficult. While the LaPC lipid went into suspension in the buffer solutions well, DPPC alone did not go into solution easily, required heating above the transition temperature for the lipid $\left(41^{\circ} \mathrm{C}\right)$, and required that the sample be kept above that temperature during the extrusion process. This was easily achieved since the extruder in lab has a water-jacketed barrel where the sample is kept and extruded. Even with heating and staying above the transition temperature, it was still difficult to synthesize the DPPC vesicles due to the extrusion process taking an abnormally long time to complete. Generally the extrusion process is quick, with only the first pass through the filters being slow. The DPPC vesicles were difficult to extrude during all 10 passes through the filters, with one sample taking over 3 hours to extrude. DLS after the extrusion of the DPPC only vesicles showed bimodal distributions in a lot of the samples prepared (Figure 3.2.4), and cleaning the extruder after the extrusion always showed signs of major lipid loss on the filter. 


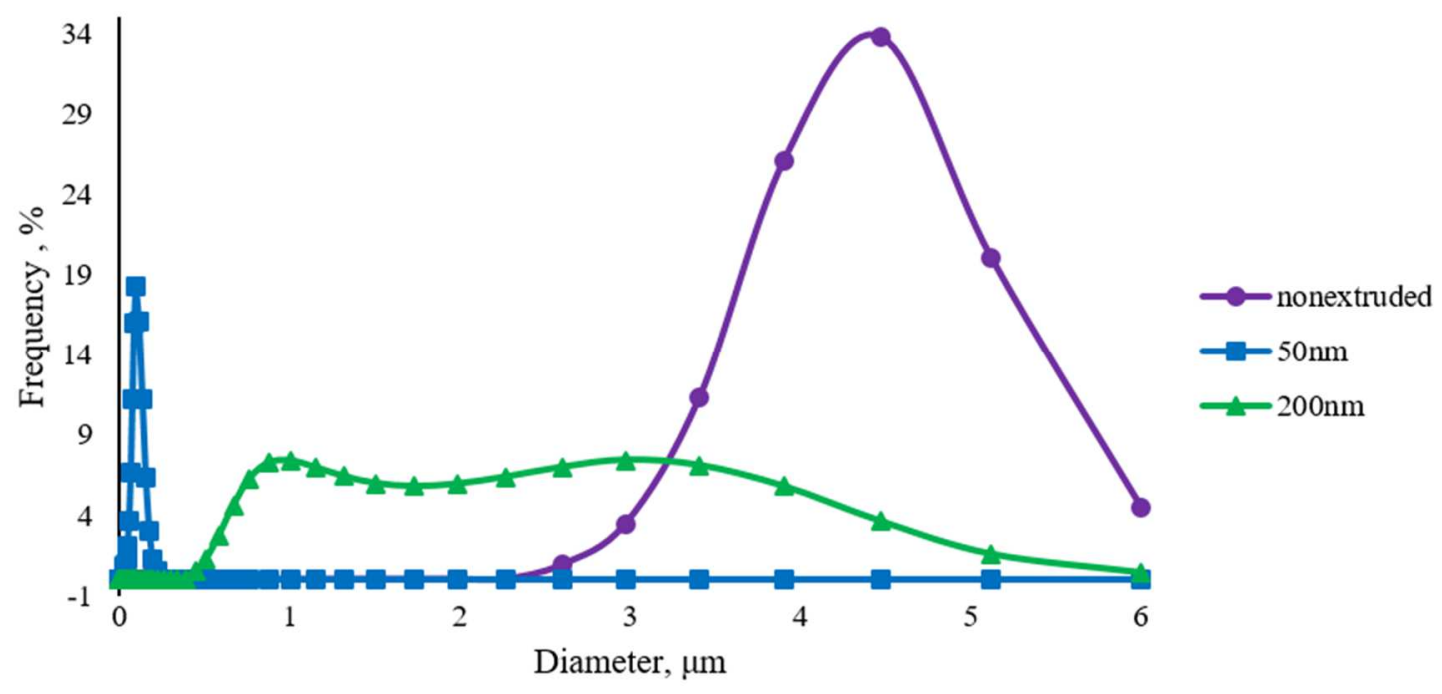

Figure 3.2.4. $5 \mathrm{mg} / \mathrm{mL}$ DPPC prepared at $43^{\circ} \mathrm{C}$, before and after extrusion at 50 and $200 \mathrm{~nm}$.

Adding cholesterol in with DPPC made the extrusion process of the synthesis easier, and did not require heating before and during the extrusion process (Figure 3.2.5). The size distributions of the DPPC $+\mathrm{C}$ vesicles were monomodal and showed homogenous sizes in the sample.

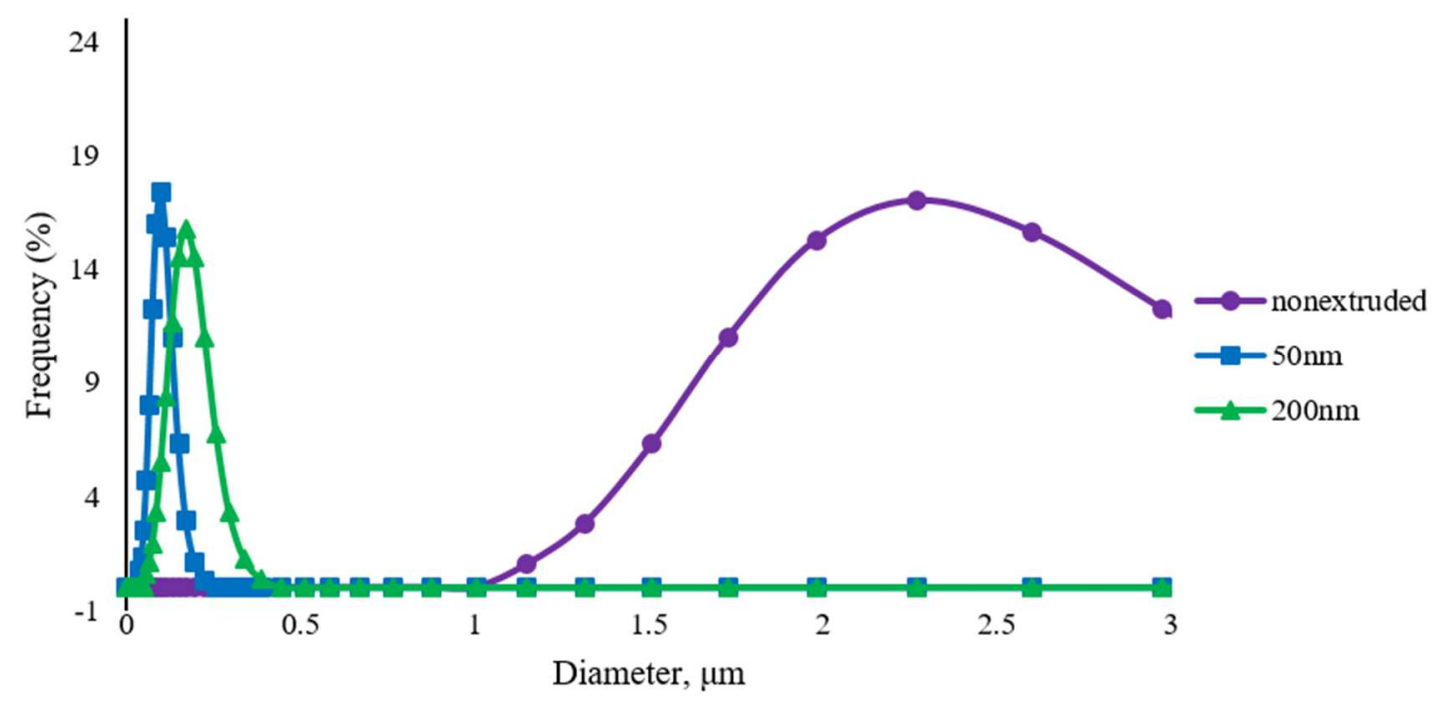

Figure 3.2.5. $5 \mathrm{mg} / \mathrm{mL}$ DPPC $+\mathrm{C}$ prepared at room temperature, before and after extrusion at 50 and 200 nm. 
However, despite efforts to keep the sample above the transition temperature and adding cholesterol into the vesicle mix, the vesicles were not stable since lipid was visibly sinking in the sample after extrusion. What this means is that the lipid was leaving the suspension and collecting at the bottom of the vials the samples were kept in. The vesicles made with DPPC were not analyzed by $\mathrm{T}_{1}$ analysis because the lipid concentration, the size of the vesicles, and the stability of the vesicles could not be guaranteed.

\section{Vesicle Size Comparisons}

Lipid size was chosen based on the size of the polycarbonate pore filters bought for the extruder. The sizes used for the $600 \mathrm{MHz}$ experiments were $50 \mathrm{~nm}, 200 \mathrm{~nm}$, and $1 \mu \mathrm{m}$. Different sized vesicles were made for the $0,1,2,3,4$, and $5 \mathrm{mM} \mathrm{GdDTPA}^{-2}$ vesicles. It was expected that the exchange rate of the vesicles would vary with changing the vesicle size. Typically, the larger the vesicle is the slower the exchange rate across the phospholipid bilayer is, and the smaller the vesicle is the faster the exchange rate is. ${ }^{236}$ However, no apparent trend can be seen when changing the size of the vesicles.

Here, all of the samples gave monoexponential fittings with $T_{1}$ values around the free GdDTPA $^{-2} \mathrm{~T}_{1}$ value for both the original and professional analysis method (Figure 3.2.6). In the original analysis method, the biexponential fittings, except for the 4 $\mathrm{mM}$ GdDTPA $^{-2}$ sample, gave one $\mathrm{T}_{1}$ value near the free $\mathrm{GdDTPA}^{-2} \mathrm{~T}_{1}$ value and one $\mathrm{T}_{1}$ value that was above $200 \mathrm{~s}$. The $\mathrm{T}_{1}$ values that were above $200 \mathrm{~s}$ all had population sizes of 0 associated with them. In the professional analysis method, the biexponential fittings all gave two $T_{1}$ values close to the free GdDTPA ${ }^{-2} T_{1}$ value and the population sizes were between $80-70 \%$ and $20-30 \%$. This data suggests that the exchange rate across the 
phospholipid bilayer is fast on the NMR timescale and that the two pools of water in the system are well mixed and that changing size of the vesicles does not change the observed $\mathrm{T}_{1}$ values. The $0 \mathrm{mM} \mathrm{GdDTPA}^{-2}$ sample provided some interesting data, and does so throughout all of the comparisons. In this case, the monoexponential fittings for both analyses, and the professional method biexponential fittings gave $\mathrm{T}_{1}$ values that were very low. It was calculated that the $\mathrm{T}_{1}$ of water would be around $3.8 \mathrm{~s}$ on the $600 \mathrm{MHz}$, but the values given by the monoexponential and the professional analysis method biexponential fittings were all well under $500 \mathrm{~ms}$. The original analysis method biexponential fitting gave one $T_{1}$ value closer to the calculated water $T_{1}$ and one value over $200 \mathrm{~s}$. The biexponential $\mathrm{T}_{1} \mathrm{~s}$ that were over $200 \mathrm{~s}$ had a population size of 0.

\section{Vesicle Concentration Comparison}

The samples in this comparison were $200 \mathrm{~nm}$ LaPC vesicles that started with a concentration of $5 \mathrm{mg} / \mathrm{mL}$ starting lipid. The vesicle samples were centrifuged down using centrifugal filter units. The sample to be concentrated was placed into the centrifugal filter unit, placed into a centrifuge at $4^{\circ} \mathrm{C}$, and spun down until the sample size was small, which typically took between 30 minutes and 4 hours depending on the amount of starting volume and how concentrated the desired sample was. The concentrated samples then had GdDTPA $^{-2}$ added to the samples for $\mathrm{T}_{1}$ analysis. The samples tested had concentrations from 0 to $5 \mathrm{mM} \mathrm{GdDTPA}^{-2}$, and $15 \mathrm{mM} \mathrm{GdDTPA}^{-2}$.

Concentrating the samples seemed to make a difference most with the original analysis biexponential fitting results (Figures 3.2.7 and 3.2.8). The monoexponential fittings for both analysis methods were similar to the free $\mathrm{GdDTPA}^{-2} \mathrm{~T}_{1}$ values, and the professional 
analysis method biexponential fittings gave $T_{1}$ values that were still close to the free GdDTPA $^{-2} \mathrm{~T}_{1}$ values, and those $\mathrm{T}_{1}$ values had populations of $80-70 \%$ and $20-30 \%$. The original analysis method biexponential fitting results generally had one $\mathrm{T}_{1}$ value near the free $\mathrm{GdDTPA}^{-2}$ and the second $\mathrm{T}_{1}$ value was either over $200 \mathrm{~s}$ or below $5 \mathrm{~s}$. The samples that were not concentrated down or just barely concentrated down had $\mathrm{T}_{1}$ values above 200 $\mathrm{s}$, and the samples that were concentrated the most had $\mathrm{T}_{1}$ values below $5 \mathrm{~s}$. Concentrating the samples also changed the population sizes, but not following a trend. The most concentrated samples had a second biexponential $\mathrm{T} 1$ value below $5 \mathrm{~s}$ that also had a population value that was not 0 . However, the population sizes were much less than those achieved with the professional analysis method.

The $0 \mathrm{mM} \mathrm{GdDTPA}^{-2}$ sample had $\mathrm{T}_{1}$ values close to the calculated $\mathrm{T}_{1}$ value for water, and seemed to benefit most from the concentration of the samples. However, there should have been no benefit since this sample should only be monoexponential with no GdDTPA $^{-2}$ anywhere in the sample. While the $T_{1}$ values obtained seemed to move in the right direction with concentration, they still were not where they were expected. 

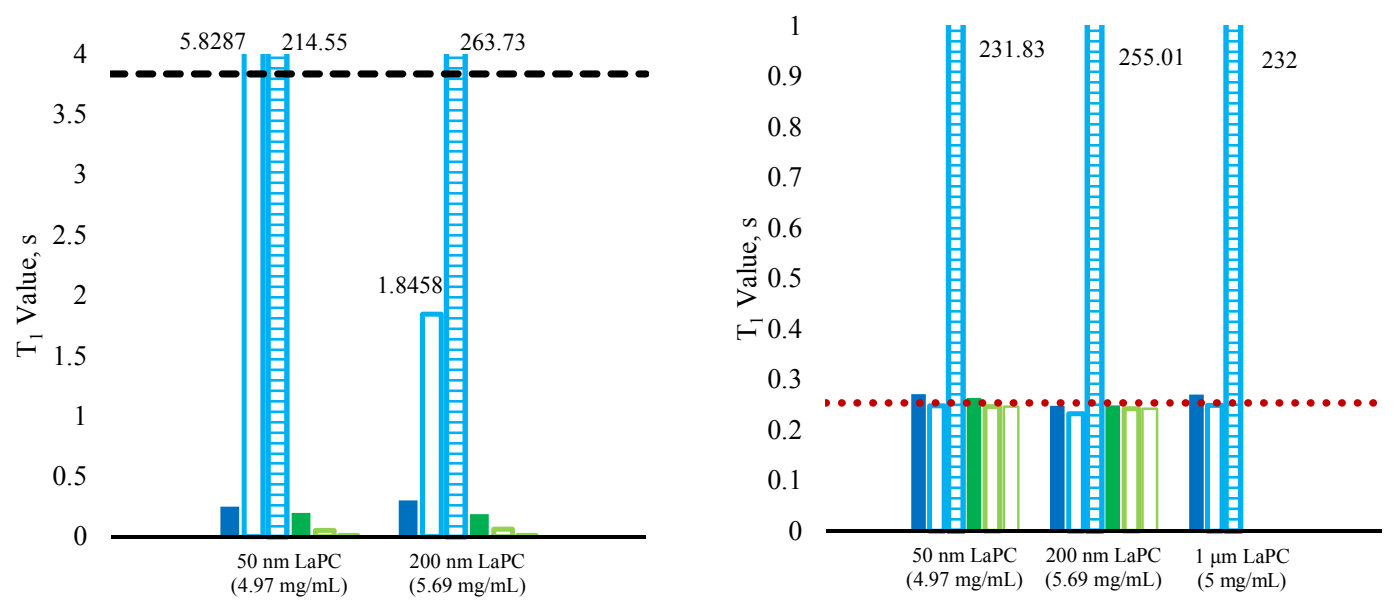

0 mM GdDTPA

LaPC Vesicles
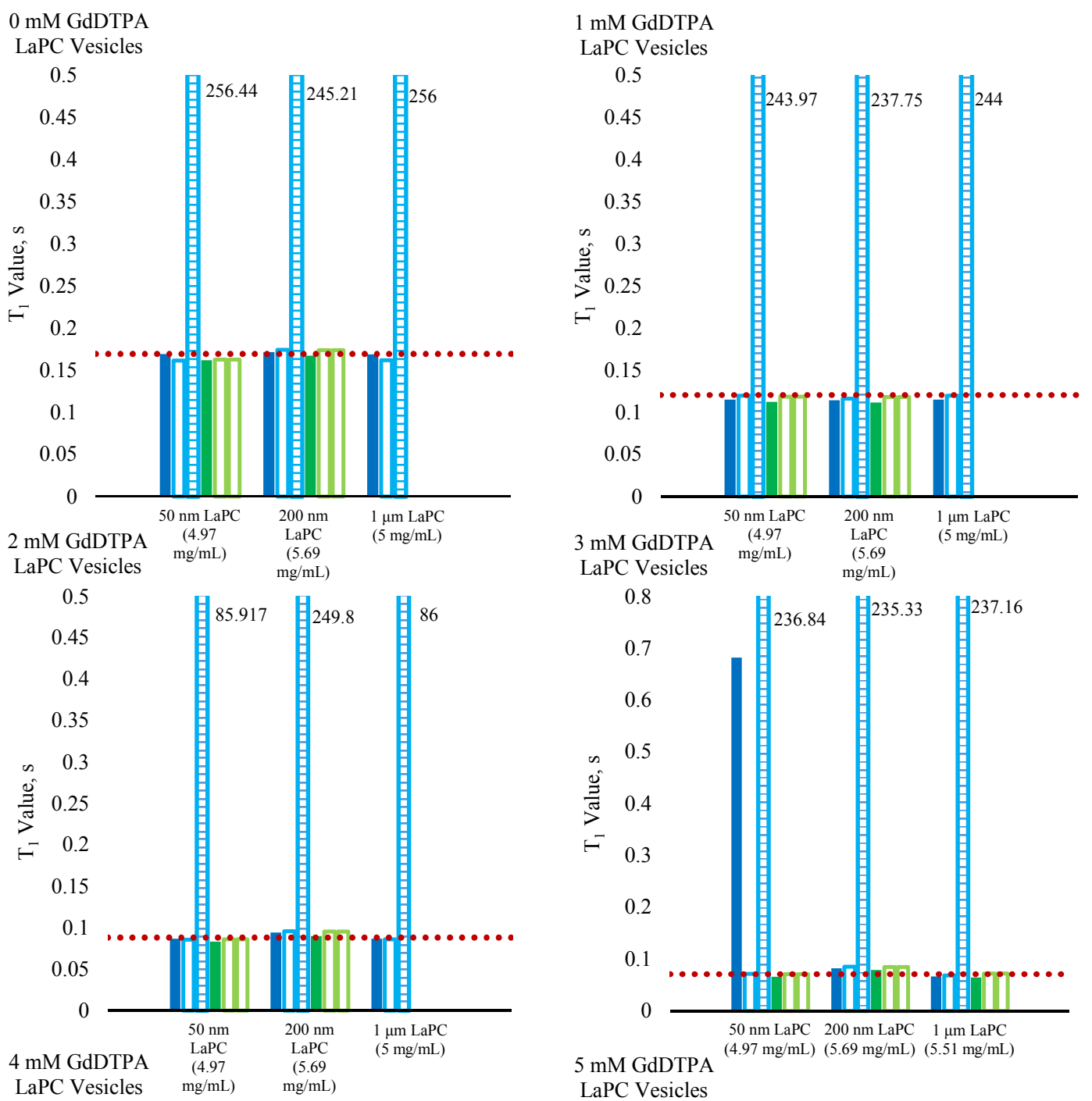

Figure 3.2.6. Size comparison $T_{1}$ values for $0-5 \mathrm{mM} \mathrm{GdDTPA}^{-2}$ samples. 


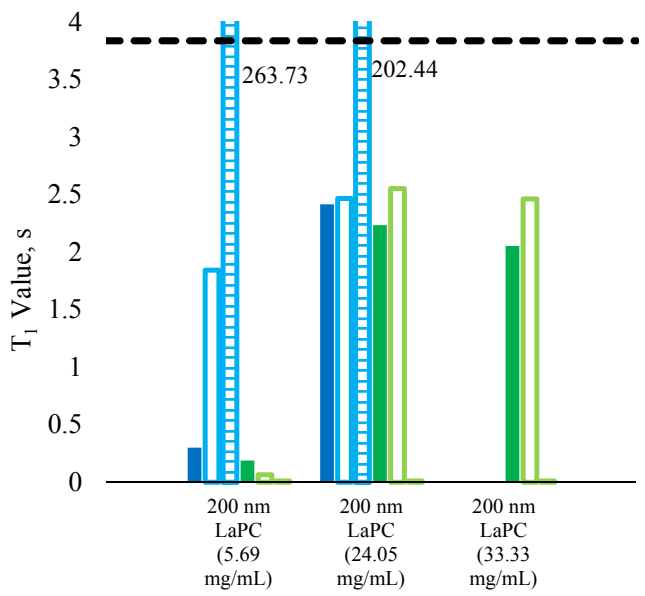

0 mM GdDTPA

LaPC Vesicles

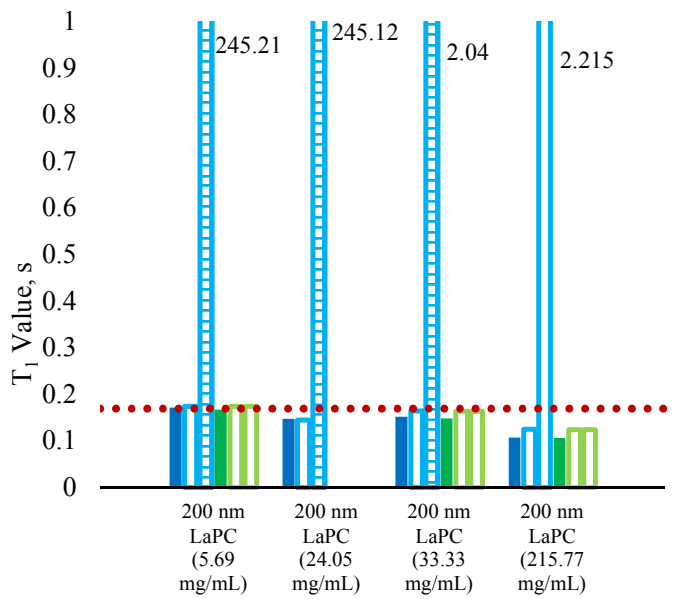

2 mM GdDTPA

LaPC Vesicles

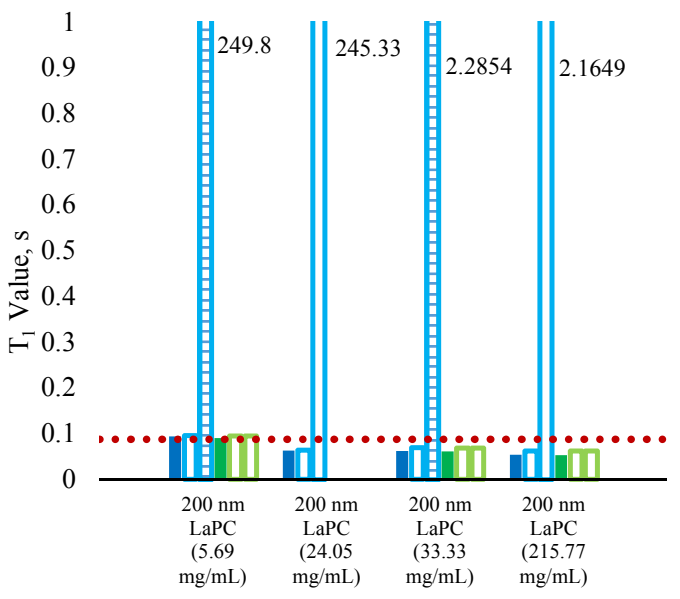

4 mM GdDTPA

LaPC Vesicles

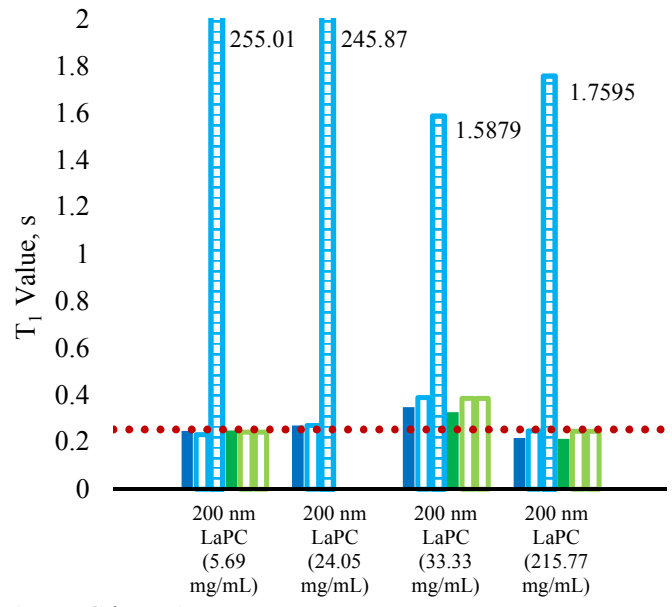

$1 \mathrm{mM}$ GdDTPA

LaPC Vesicles

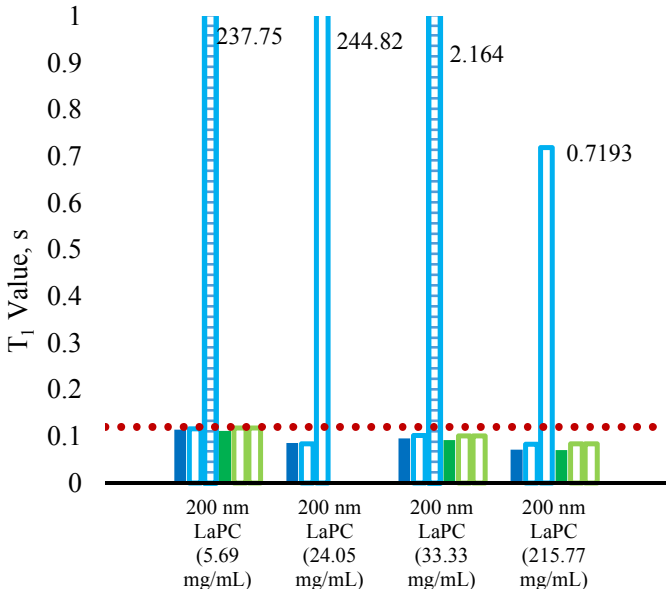

3 mM GdDTPA

LaPC Vesicles

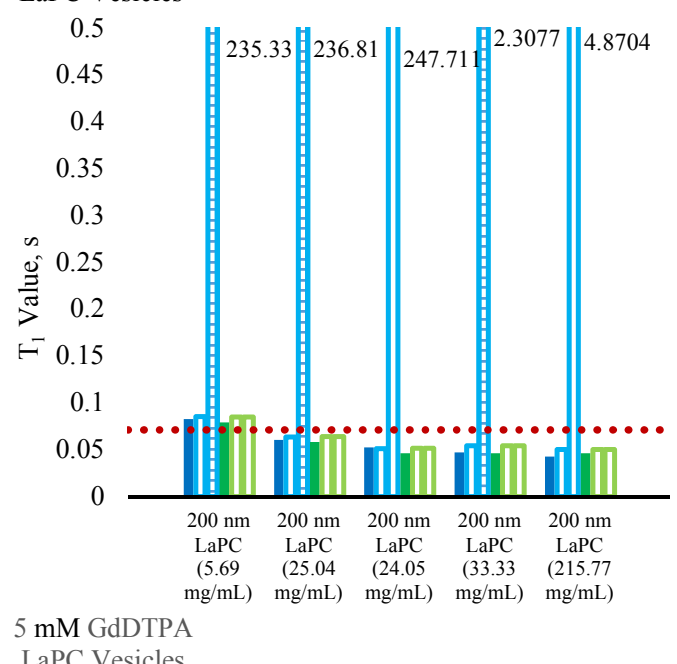

Figure 3.2.7. Vesicle concentration comparison $T_{1}$ values for $0-5 \mathrm{mM} \mathrm{GdDTPA}^{-2}$ samples. 


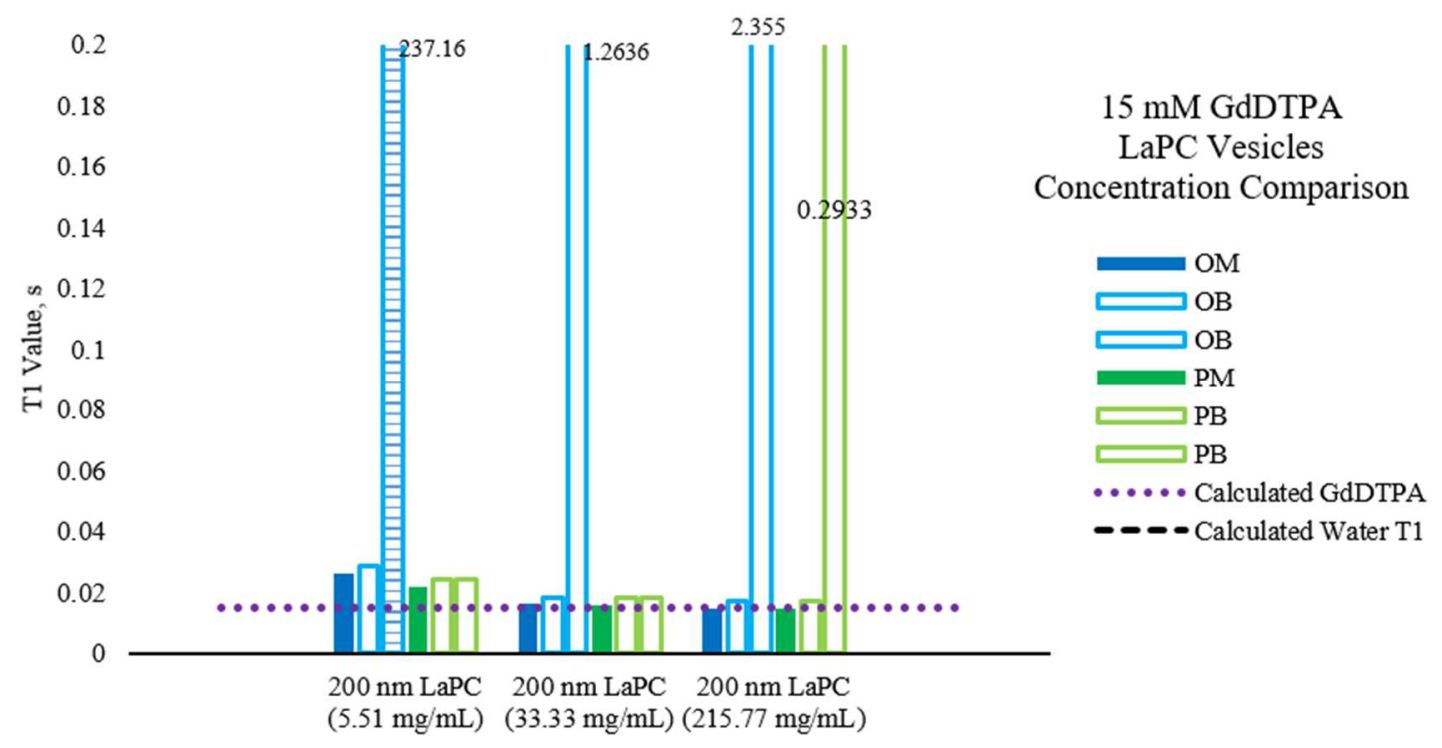

Figure 3.2.8. Vesicle concentration comparison $T_{1}$ values for $15 \mathrm{mM} \mathrm{GdDTPA}^{-2}$ samples

\section{Vesicle Formation Comparison}

The original vesical formation technique employed just extrusion. This methodology was supposed to form ULVs while making the size distribution homogenous within the sample. In order to make sure the ULVs were being made, a freeze-thaw cycle was added to the vesicle synthesis. The freeze thaw cycles involved flash freezing the vesicles in a liquid nitrogen bath and then thawing them out in a heated water bath. This method is supposed to produce only ULVs and not MLVs. The idea here is that the frozen water breaks up the smaller vesicles inside the larger vesicles and forces the lipid from the smaller vesicles to join the larger vesicle upon thawing. 

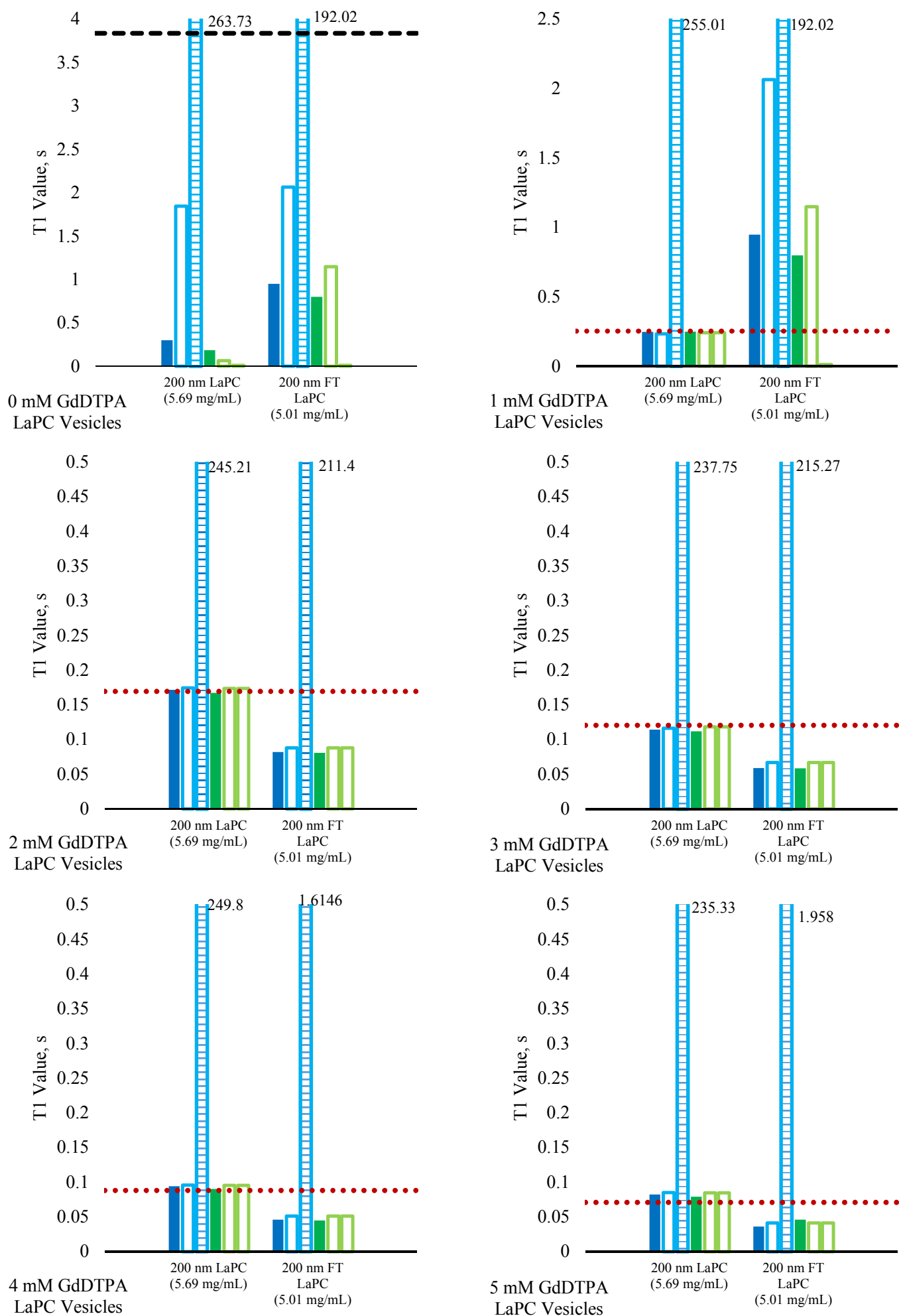

Figure 3.2.9. Formation method comparison $T_{1}$ values for $0-5 \mathrm{mM} \mathrm{GdDTPA}^{-2}$ samples. 


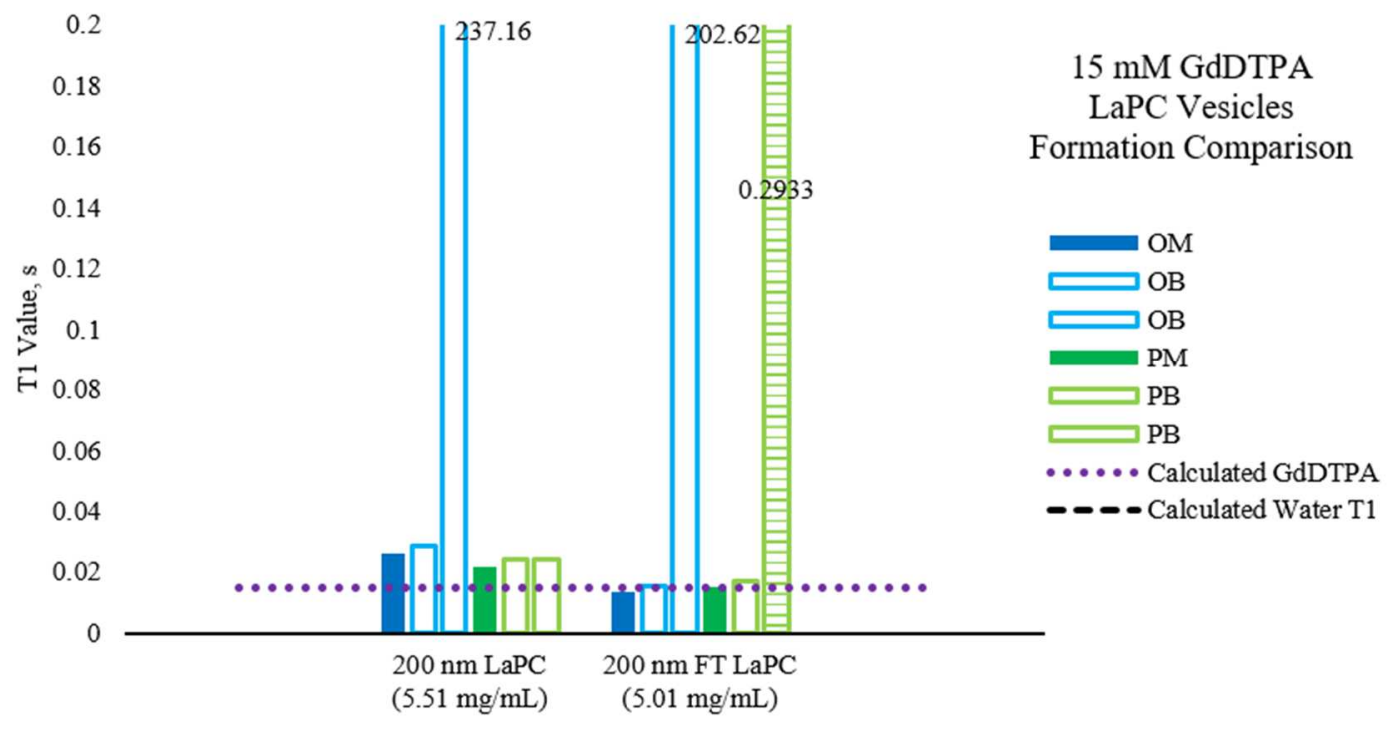

Figure 3.2.10. Formation method comparison $T_{1}$ values for $15 \mathrm{mM} \mathrm{GdDTPA}^{-2}$ samples.

In the normal vesicle formation, the $6 \mathrm{~T}_{1}$ values collected follow the trends already discussed (Figures 3.2.9 and 3.2.10). With the exception of the $0 \mathrm{mM} \mathrm{GdDTPA}^{-2}$ sample, the monoexponential fittings and the professional analysis method biexponential fittings were near the free $\mathrm{GdDTPA}^{-2} \mathrm{~T}_{1}$ value, and the original analysis method biexponential fittings had one $T_{1}$ value near the free or calculated GdDTPA ${ }^{-2} T_{1}$ value and the other $T_{1}$ value was over $200 \mathrm{~s}$. However, the results from changing the vesicle formation have a trend unlike any of the other data comparisons. The $\mathrm{T}_{1}$ values in all of the original $200 \mathrm{~nm}$ LaPC vesicles with 5.69 or $5.51 \mathrm{mg} / \mathrm{mL}$ initial concentration of lipid, except for the 0 and $1 \mathrm{mM} \mathrm{GdDTPA}^{-2}$ samples, are all larger than those found for the $200 \mathrm{~nm}$ FT LaPC vesicles with $5.01 \mathrm{mg} / \mathrm{mL}$ initial concentration of lipid. The values follow the same trend as the original vesicles, but with smaller $T_{1}$ values given from the fittings. The reason for this is unknown. This could be a result of there only being one compartment in each vesicle. 

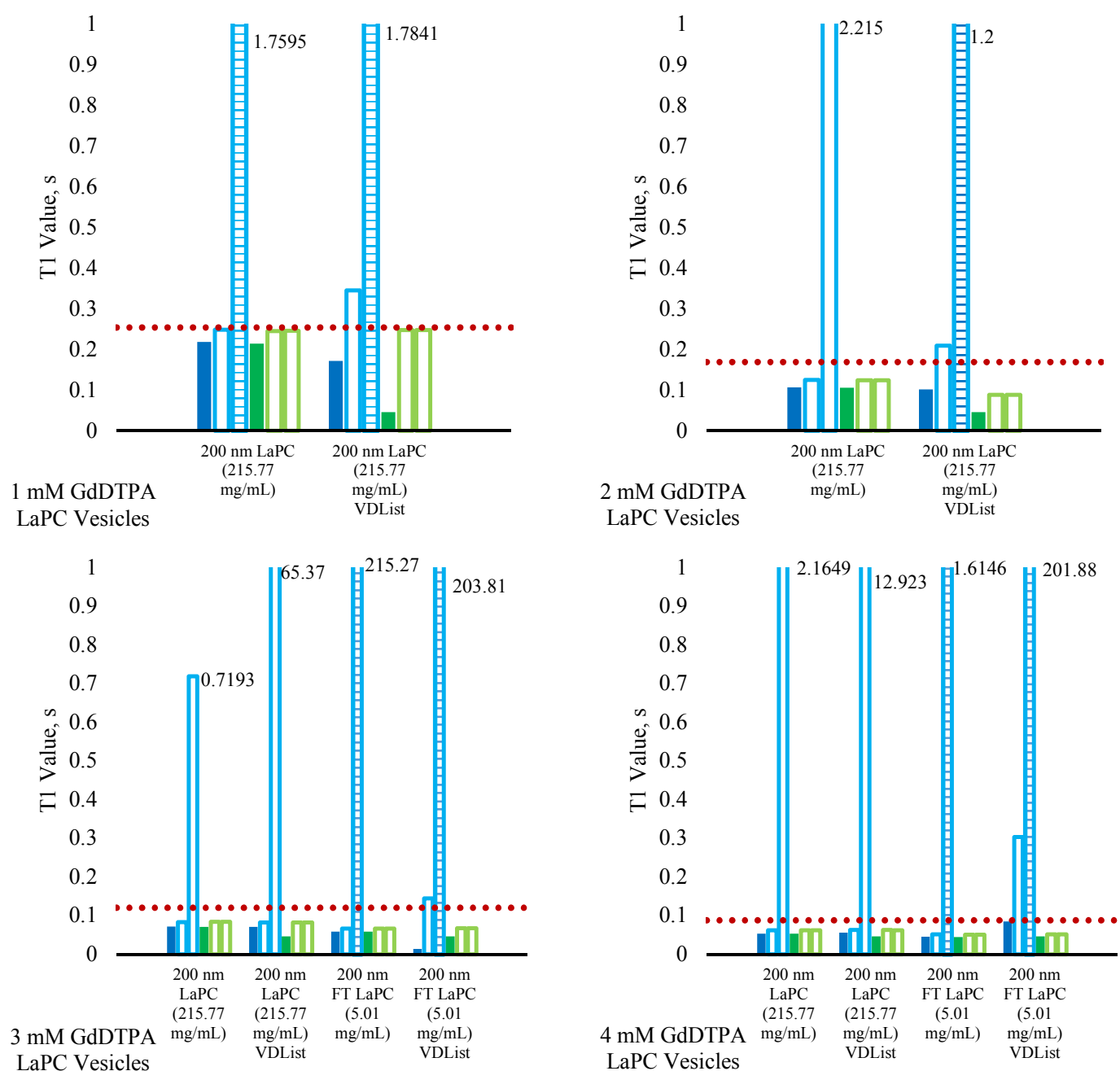

LaPC Vesicles
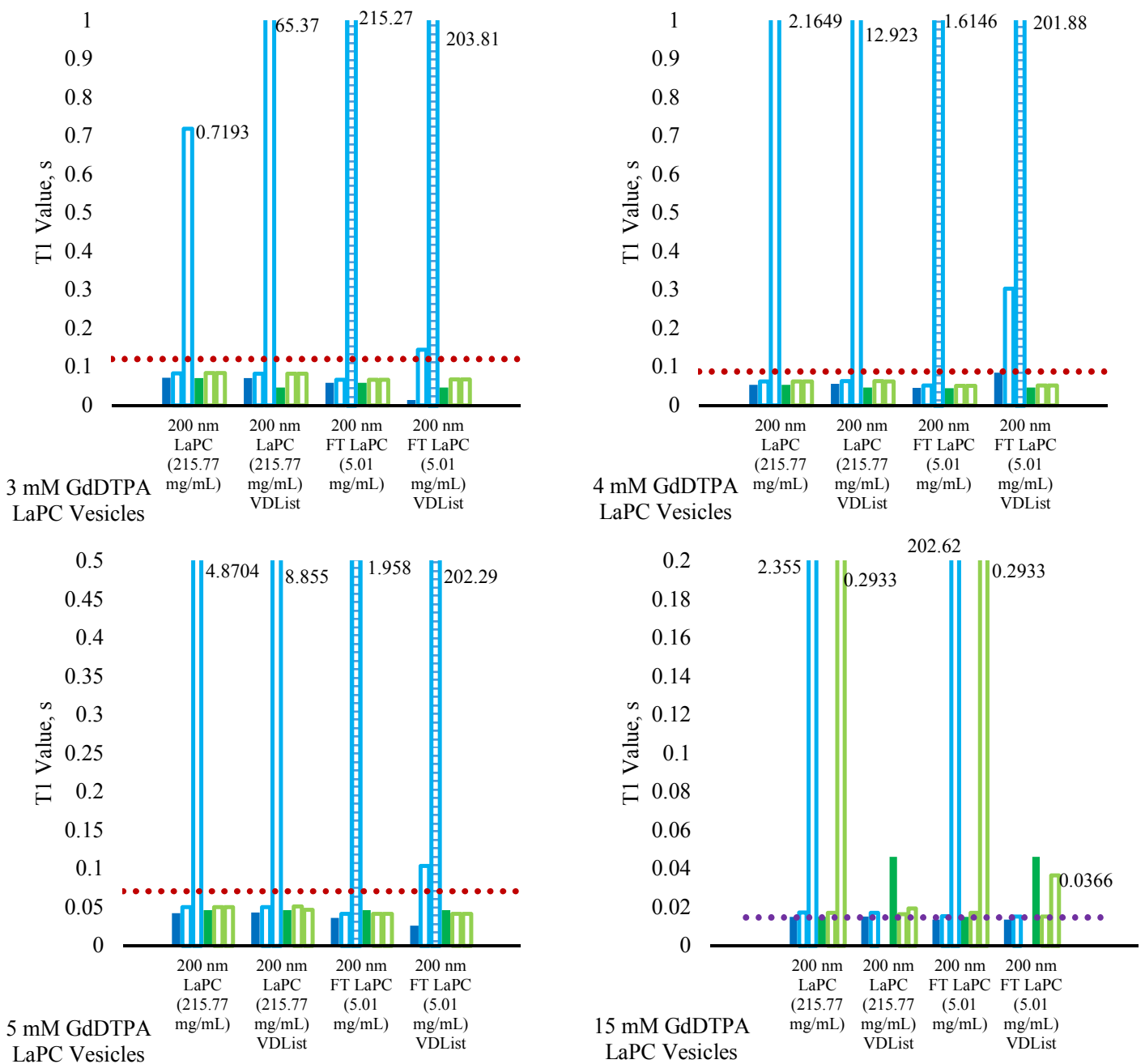

Figure 3.2.11. Data collection comparison $T_{1}$ values for $1-5$ and $15 \mathrm{mM} \mathrm{GdDTPA}^{-2}$ samples. 
Previously, encapsulated $\mathrm{GdDTPA}^{-2}$ was found to have a lower $\mathrm{T}_{1}$ value than free GdDTPA $^{-2237,238}$, and it was suggested that this was due to a decrease in the exchange rate between bulk water and the entrapped GdDPTA ${ }^{-2}$. In this case, the GdDTPA ${ }^{-2}$ is on the outside of the vesicle, not on the inside and encapsulated, but the result should still be the same since the chelate isn't seeing all of the water in the system and therefore the relaxivity is limited and lower than when the chelate isn't around vesicles, or compartments. However, it has been shown previously that there is fast exchange kinetics when GdDTPA $^{-2}$ is in the presence of whole blood and frog heart tissue and that $T_{1}$ values do not suffer a decrease in intensity ${ }^{239}$. The variable reports from previous studies make knowing what to expect difficult.

\section{Data Collection Comparison}

The original samples were run with their respective concentration dependent VD lists, and samples labelled with "VD List" in the name were also run with a concentration independent VD list (see Chapter 4 for more information). No real trends were discernable in the data collection analysis (Figure 3.2.11). This comparison seems to be the one with the least amount of a trend available with the data collected. Including all of the signal in the data collection does not seem to make the biexponential nature of the system more readily apparent. The $15 \mathrm{mM} \mathrm{GdDTPA}{ }^{-2}$ sample appeared to have a change in the professional analysis method, but other than that, no trends were observed.

\section{Finding the Encapsulated Volume of $100 \mathrm{~nm}$ Vesicles}

Shift reagent experiments had previously been attempted to find the encapsulated volume of vesicle batches. However, the encapsulated shift reagent was never visible, 
presumably because of the buffer showing in ${ }^{1} \mathrm{H}$ NMRs. Even with the buffer removed from the samples and the concentration of the shift reagent increased, the volume with the shift reagent, whether inside or outside of the vesicles, was never visible on the ${ }^{1} \mathrm{H}$ NMRs taken. Therefore, geometry was used to estimate the amount of encapsulated water in the vesicle systems.

Given that the vesicle is a $100 \mathrm{~nm}$ vesicle, the external diameter is $100 \mathrm{~nm}$ and external radius is $50 \mathrm{~nm}$. If the lipid wall thickness is $5 \mathrm{~nm}$, then the internal diameter is $90 \mathrm{~nm}$ and the internal radius is $45 \mathrm{~nm}$. Both the internal area of the vesicle and the external area of the vesicle are given by the following equation:

$$
A=4 \pi r^{2}
$$

Where $\mathrm{A}$ is the area and $\mathrm{r}$ is radius. Therefore, the internal area of the vesicle is $25,446.9$ $\mathrm{nm}^{2}$ and the external area of the vesicle is $31,415.93 \mathrm{~nm}^{2}$. If a choline head group area is $0.70 \mathrm{~nm}^{2} /$ lipid head, then the number of head groups on the inside of the vesicle is $36,352.715$ and the number of head groups on the outside of the vesicle is $44,879.895$. If those two values are added together, the total number of head groups in one vesicle, and therefore the number of lipid molecules in one vesicle, is $81,232.610$. Rounding this number up to a whole value gives a total number of 81,233 lipids required to make up one vesicle.

Since the general concentration of lipid used was $5 \mathrm{mg} / \mathrm{mL}$, this gives a total of $0.05 \mathrm{~g}$ of lipid in a $10 \mathrm{~mL}$ sample. Using the average molecular weight of LaPC (768 g/mol), the number of moles of LaPC in a $10 \mathrm{~mL} 5 \mathrm{mg} / \mathrm{mL}$ sample is $6.5 \times 10^{-5}$ moles. Using Avogadro's number, the number of lipids becomes $3.92 \times 10^{19}$. Dividing this value by the number of 
lipids in one vesicle give the number of vesicles present in this specific sample, which is $4.826 \times 10^{14}$ vesicles. Multiplying the number of vesicles in a sample by the internal volume of a vesicle gives the total internal volume in one sample, which is given by the following equation:

$$
V=\frac{4}{3} \pi r^{3}
$$

Where $\mathrm{V}$ is the volume and $\mathrm{r}$ is the radius. The internal volume of a $100 \mathrm{~nm}$ vesicle is $1.8437 \times 10^{20} \mathrm{~nm}^{3}$. Converted, this value comes out to $0.18437 \mathrm{~mL}$ of internal volume per every $10 \mathrm{~mL}$ total volume of $5 \mathrm{mg} / \mathrm{mL} 100 \mathrm{~nm}$ vesicle sample.

In order for the $\mathrm{T}_{1}$ analysis to work, at least $10 \%$ of the signal should be internal, and in the present system, only $1.8 \%$ of the total volume is encapsulated right now. Even with concentrating down the samples to decrease the external volume and increase the ratio of encapsulated volume, the samples still aren't concentrated enough, and there is no guarantee that the samples that are concentrated down aren't destroying the vesicles at the same time. Without imaging the vesicles, there is no way to know whether or not the technique of concentrating samples is destructive.

\subsection{CEST Analysis of Vesicle Systems}

\section{Preliminary Samples}

A CEST response can be measured for a sample with 3-4\% signal, therefore the analysis method was changed from a $\mathrm{T}_{1}$ analysis to a CEST analysis. In this technique, the vesicles were synthesized in the presence of $150 \mathrm{mM} \mathrm{TmEDTA}{ }^{-1}$ and $150 \mathrm{mM}$ TmDTPA $^{-2}$. The vesicles formed were either LaPC only or LaPC and cholesterol and the samples were run at $25^{\circ} \mathrm{C}$ and $5^{\circ} \mathrm{C}$. If exchange across the membrane were slow enough, a 
CEST signal would appear at the same frequency as the shifted protons. If exchange were too fast across the membrane, no CEST signal would be observed.

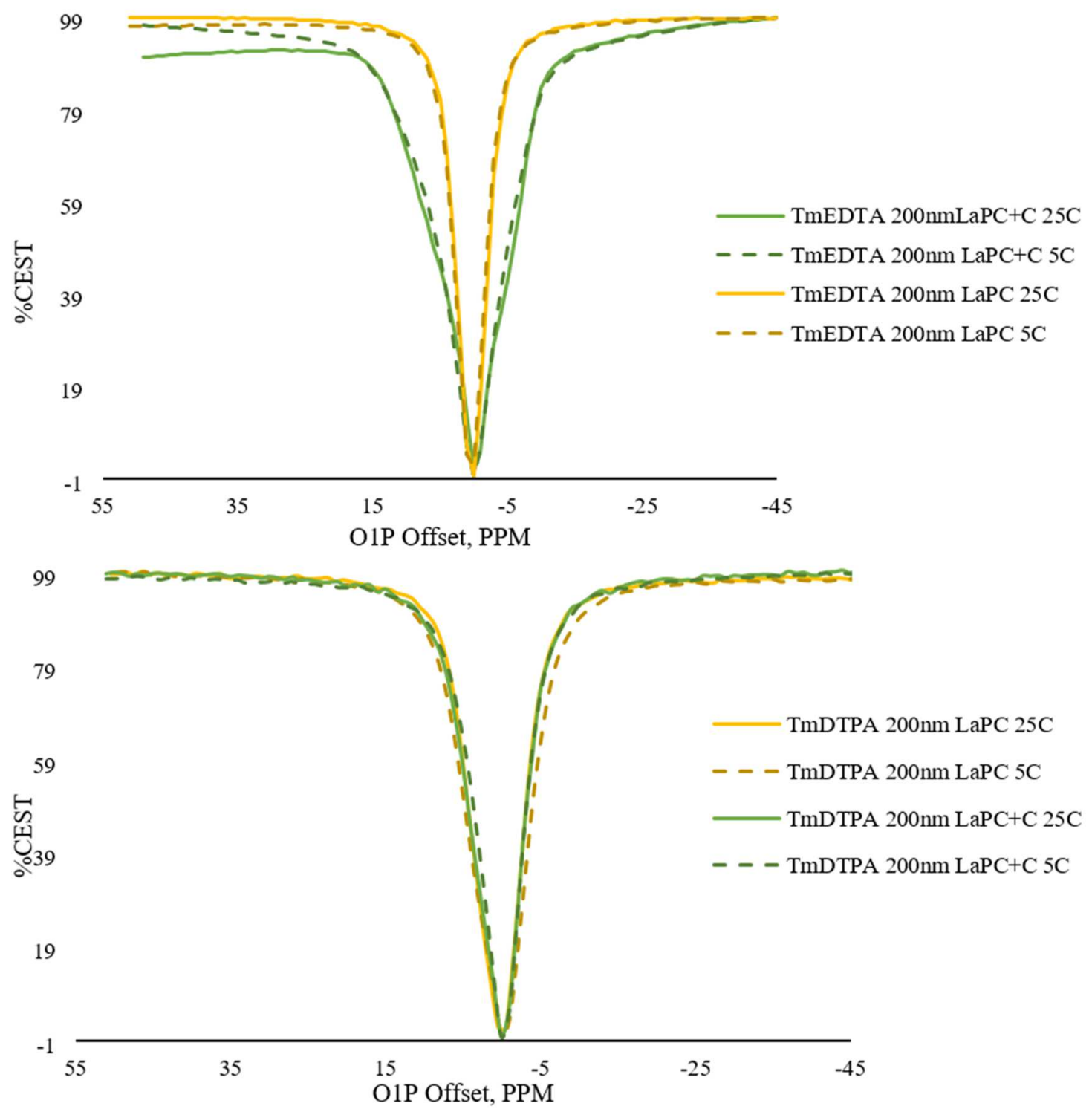

Figure 3.3.1. Preliminary CEST spectra on vesicle samples. Top: $150 \mathrm{mM} \mathrm{TmEDTA}{ }^{-1}$ samples. Bottom: $150 \mathrm{mM}$ TmDTPA ${ }^{-2}$ samples. Green lines represent LaPC $+\mathrm{C}$ samples. Yellow lines represent LaPC only vesicles. Solid lines represent room temperature experiments. Dashed lines represent low temperature experiments.

The TmDTPA ${ }^{-2}$ samples had no CEST signal appear and the TmEDTA ${ }^{-1}$ LaPC vesicles had no CEST signal; however, the TmEDTA $^{-1}$ LaPC and cholesterol vesicles had asymmetry in their spectrum, associated with CEST signal (Figure 3.3.1). These were 
preliminary data collected at low concentrations of shift reagent, so further experimentation was done in a more thorough study, including control compounds.

\section{Controlled Samples}

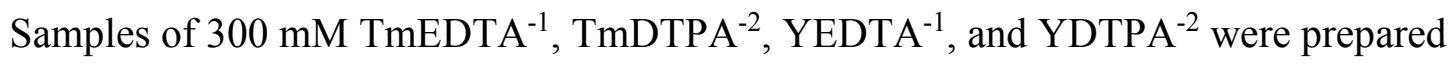
as buffer solutions for vesicle formation. Vesicles were formed for the $\mathrm{TmDTPA}^{-2}$ and YDTPA $^{-2}$ samples, but vesicles were not able to form using the TmEDTA ${ }^{-1}$ and YEDTA $^{-1}$ samples. This was likely due to the fact that the EDTA being used was old and not fully dry, leading to an uncertainty in the true amount of EDTA being added and resulting in free $\mathrm{Ln}^{3+}$ ions being present in the solutions. Free $\mathrm{Ln}^{3+}$ ions make the formation of vesicles difficult by coordinating with the polar head groups of the lipids. Therefore, only the 300 $\mathrm{mM} \mathrm{TmDTPA}^{-2}$ and YDTPA ${ }^{-2}$ samples are presented. In order to know where to look in the collected spectra, and shift experiment was performed where $2 \mathrm{M} \mathrm{TmDTPA}^{-2}$ was diluted down in steps and ${ }^{1} \mathrm{H}$ NMR spectra were run to see where the shifted protons of the encapsulated water would occur (Figure 3.3.2). The YDTPA ${ }^{-2}$ chelate is not a shift reagent, so no ${ }^{1} \mathrm{H}$ NMR shift experiments were performed for it. The YDTPA $^{-2}$ samples serve as controls for the $\mathrm{TmDTPA}^{-2}$ samples since the $\mathrm{YDTPA}^{-2}$ chelate will be similar in its coordination chemistry and geometry to the $\mathrm{TmDTPA}^{-2}$ chelate but will not have two chemically different pools of water like the TmDTPA-2 samples will have. 


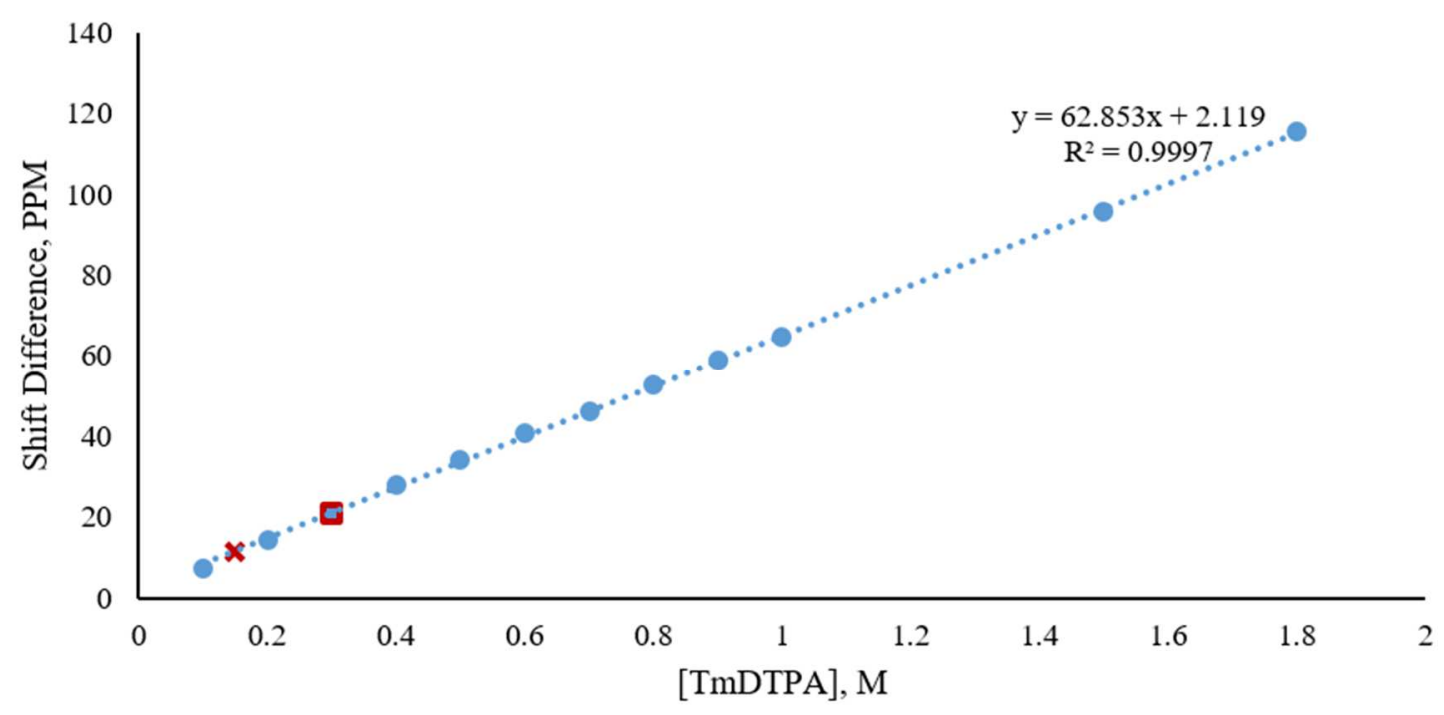

Figure 3.3.2. TmDTPA $^{-2}$ concentration shift dependence experiment.

The shifting ability of the $150 \mathrm{mM} \mathrm{TmDTPA}^{-2}$ for the first experiments at $150 \mathrm{mM}$ is marked by a red $\mathrm{x}$, and the shifting ability of the $300 \mathrm{mM} \mathrm{TmDTPA}^{-2}$ for the following experiments is marked by a red square (Figure 3.3.2). In the $150 \mathrm{mM} \mathrm{TmDTPA}^{-2}$ samples, the shifted CEST peak should have been around 11 ppm, while in the $300 \mathrm{mM} \mathrm{TmDTPA}^{-}$ ${ }^{2}$ samples, the shifted CEST peak should have been around $20 \mathrm{ppm}$. The TmDTPA ${ }^{-2}$ and YDTPA $^{-2}$ vesicle samples were run at varying pulse power levels (PL) in order to assess how the CEST signal would change with power. If the \%CEST signal changed with the pulse power then it would be considered a true CEST signal and not an artifact from the sample or instrument. On the $600 \mathrm{MHz}$ spectrometer, as the pulse power level number increases, the actual pulse power decreases, and vice versa. Therefore, a pulse power level (PL) of 30 is actually a lower pulse power level than 12 . 


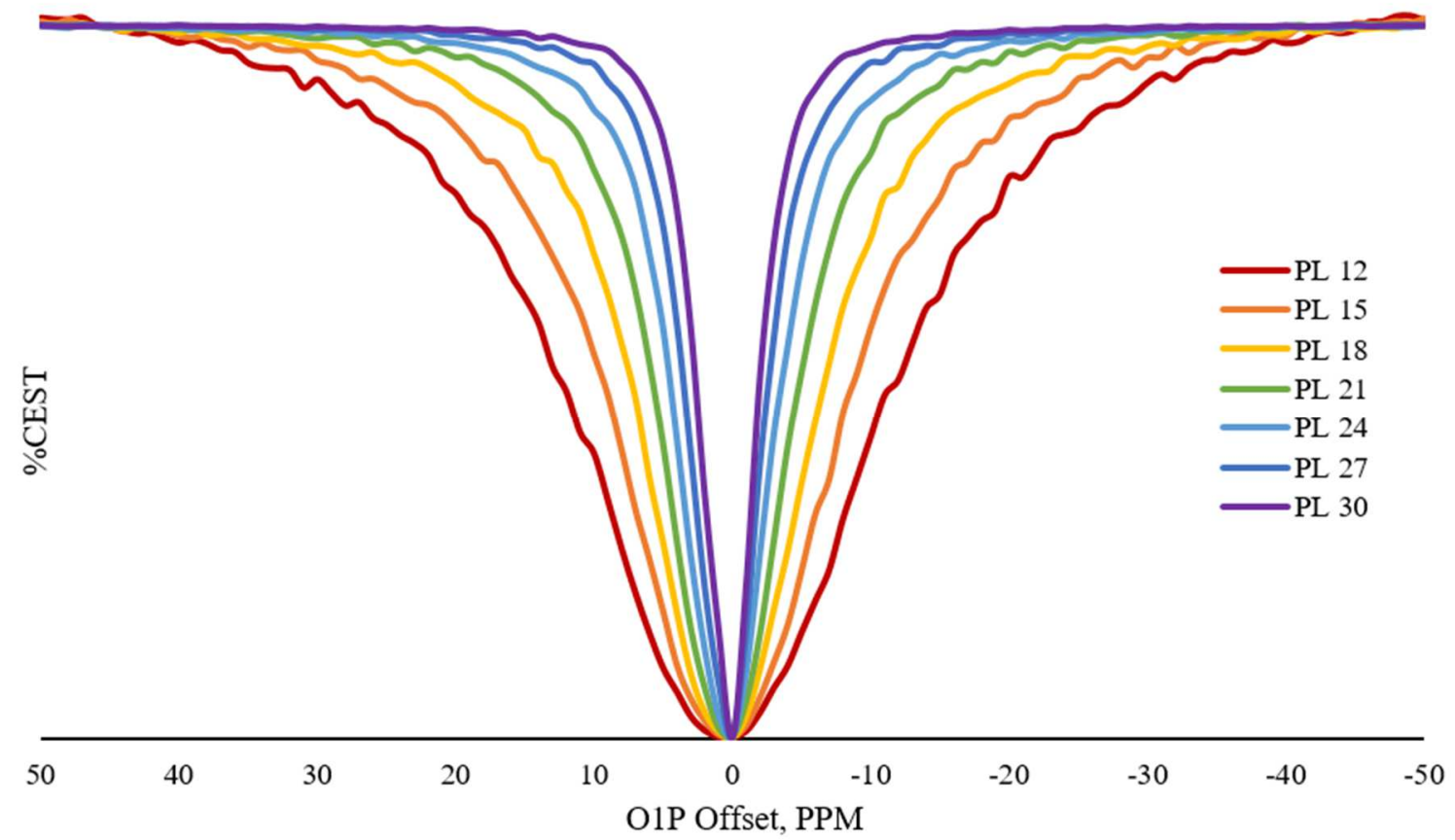

Figure 3.3.3. $300 \mathrm{mM} \mathrm{TmDTPA} \mathrm{m}^{-2} 200 \mathrm{~nm}$ LaPC vesicle CEST spectra.

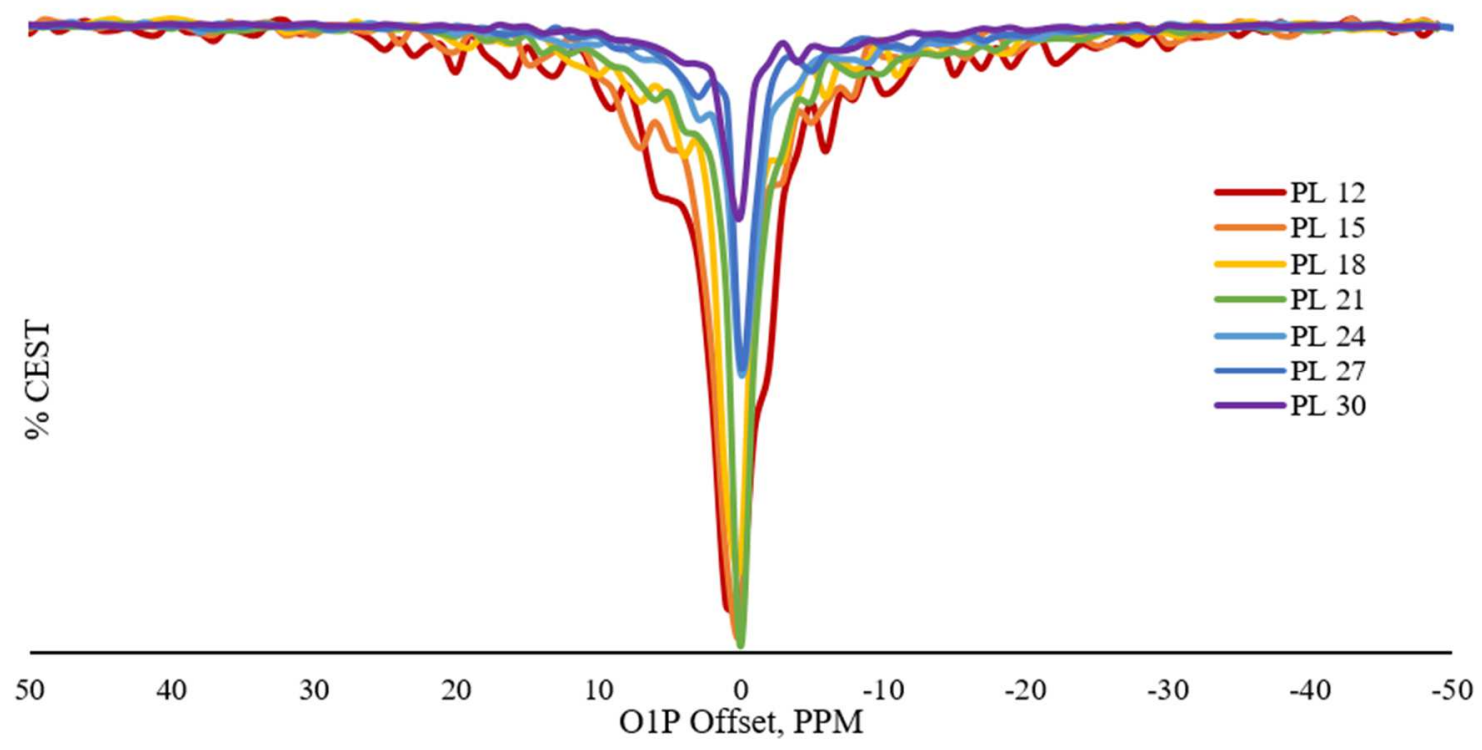

Figure 3.3.4. $300 \mathrm{mM} \mathrm{YDTPA}^{-2} 200 \mathrm{~nm}$ LaPC vesicle CEST spectra.

The $300 \mathrm{mM}$ TmDTPA ${ }^{-2}$ LaPC only vesicles did not produce any CEST signal, other than slight asymmetry and broadened spectra (Figure 3.3.3). The $300 \mathrm{mM} \mathrm{YDTPA}^{-2}$ LaPC only vesicles had narrow water saturation peaks (Figure 3.3.4), which was expected since the water inside and outside of the vesicles was chemically equivalent. The broadened 
nature of the TmDPTA $^{-2}$ sample could be an indication of multiple chemically nonequivalent environments, which is a result of compartmentalization and means that the vesicles are most likely not unilamellar.

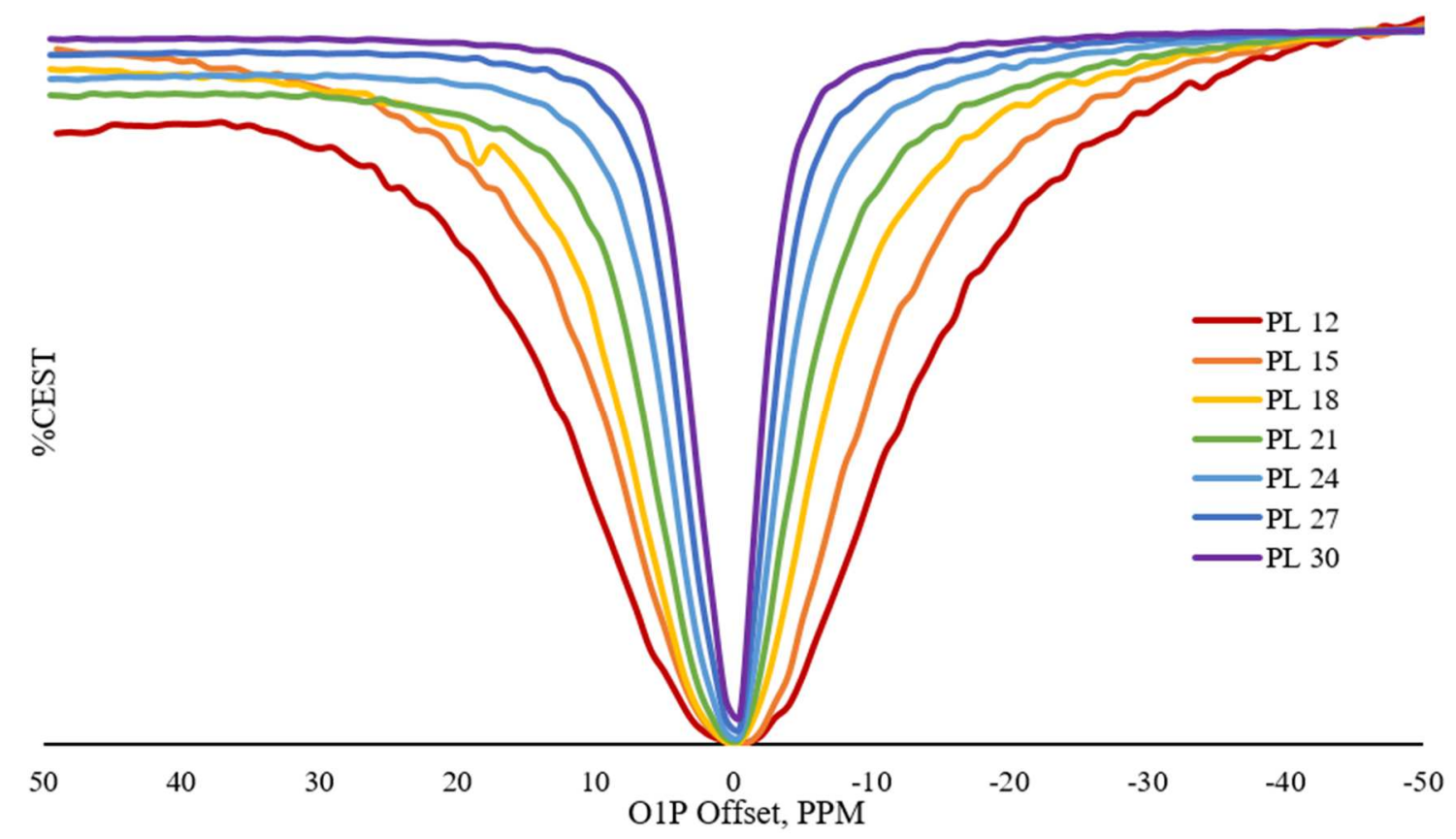

Figure 3.3.5. $300 \mathrm{mM} \mathrm{TmDTPA}{ }^{-2} 200 \mathrm{~nm} \mathrm{LaPC}+\mathrm{C}$ vesicle CEST spectra.

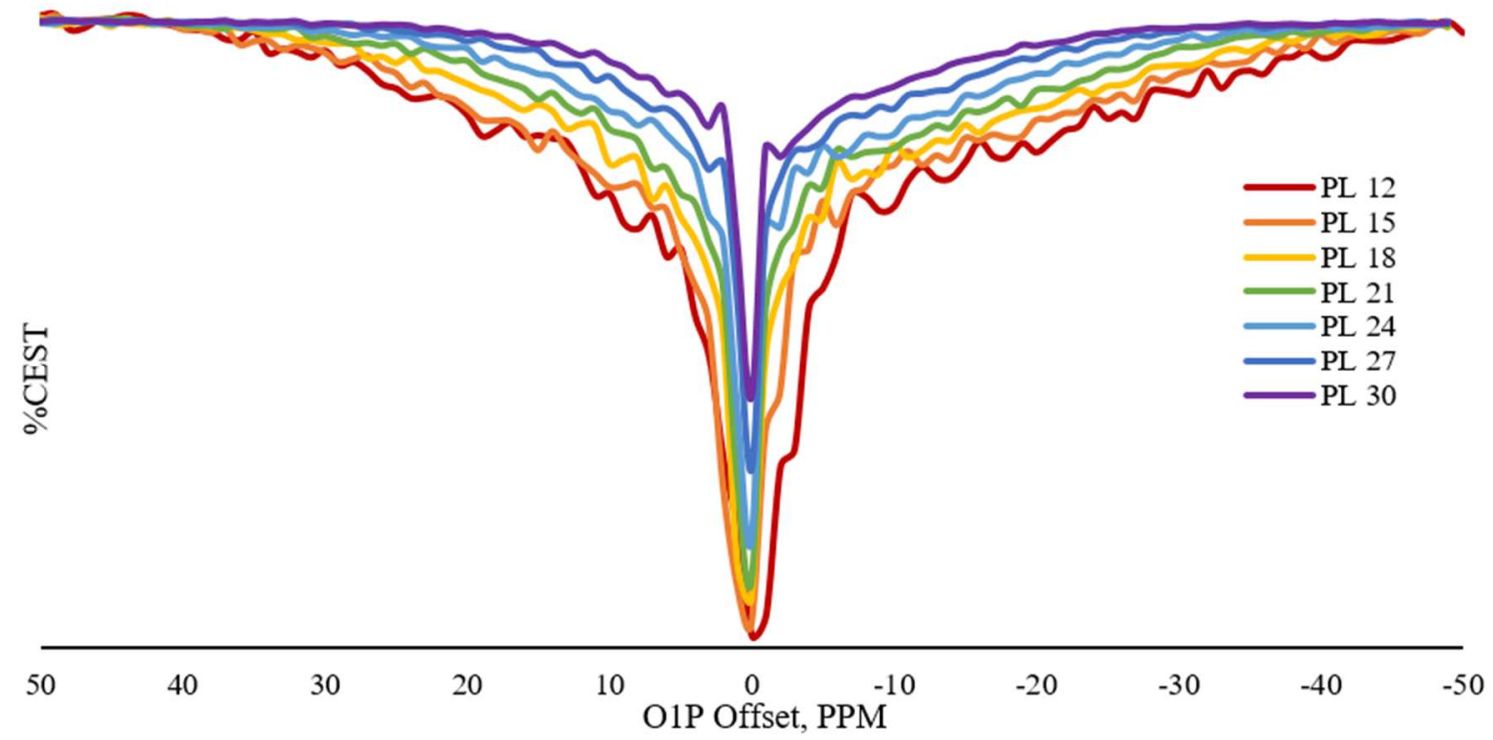

Figure 3.3.6. $300 \mathrm{mM} \mathrm{YDTPA}^{-2} 200 \mathrm{~nm} \mathrm{LaPC}+\mathrm{C}$ vesicle CEST spectra. 
The $300 \mathrm{mM} \mathrm{TmDTPA}^{-2} 200 \mathrm{~nm} \mathrm{LaPC}+\mathrm{C}$ vesicles also did not produce any CEST signal, aside from a general broadening of the direct water saturation (Figure 3.3.5). The $\mathrm{LaPC}+\mathrm{C}$ TmDTPA ${ }^{-2}$ sample was broader than the LaPC only $\mathrm{TmDTPA}^{-2}$ sample, which is expected due to the fact that the water exchange rate in the cholesterol system should be longer. Again, the $300 \mathrm{mM} \mathrm{YDTPA}^{-2} \mathrm{LaPC}+\mathrm{C}$ vesicles had narrow water saturation peaks (Figure 3.3.6) similar to the LaPC only vesicle sample.

The spectra collected are difficult to assess due to the control sample spectra not coming out like they should. The YDTPA-2 spectra (Figures 3.3.4 and 3.36) look as though the experiment was not set up properly, with either the FID not collected fully or the O1P value not being set properly. Given that both of the YDTPA ${ }^{-2}$ samples had noninterpretable data, but both of the TmDTPA ${ }^{-2}$ samples were interpretable, the experimental set up being the problem is not likely. The TmDTPA ${ }^{-2}$ samples had slight asymmetry associated with them (Figure 3.3.7) when compared to the YDTPA ${ }^{-2}$ samples, but within a couple ppm of O1P where the CEST signal from $300 \mathrm{mM} \mathrm{TmDTPA}^{-2}$ should not have been. 

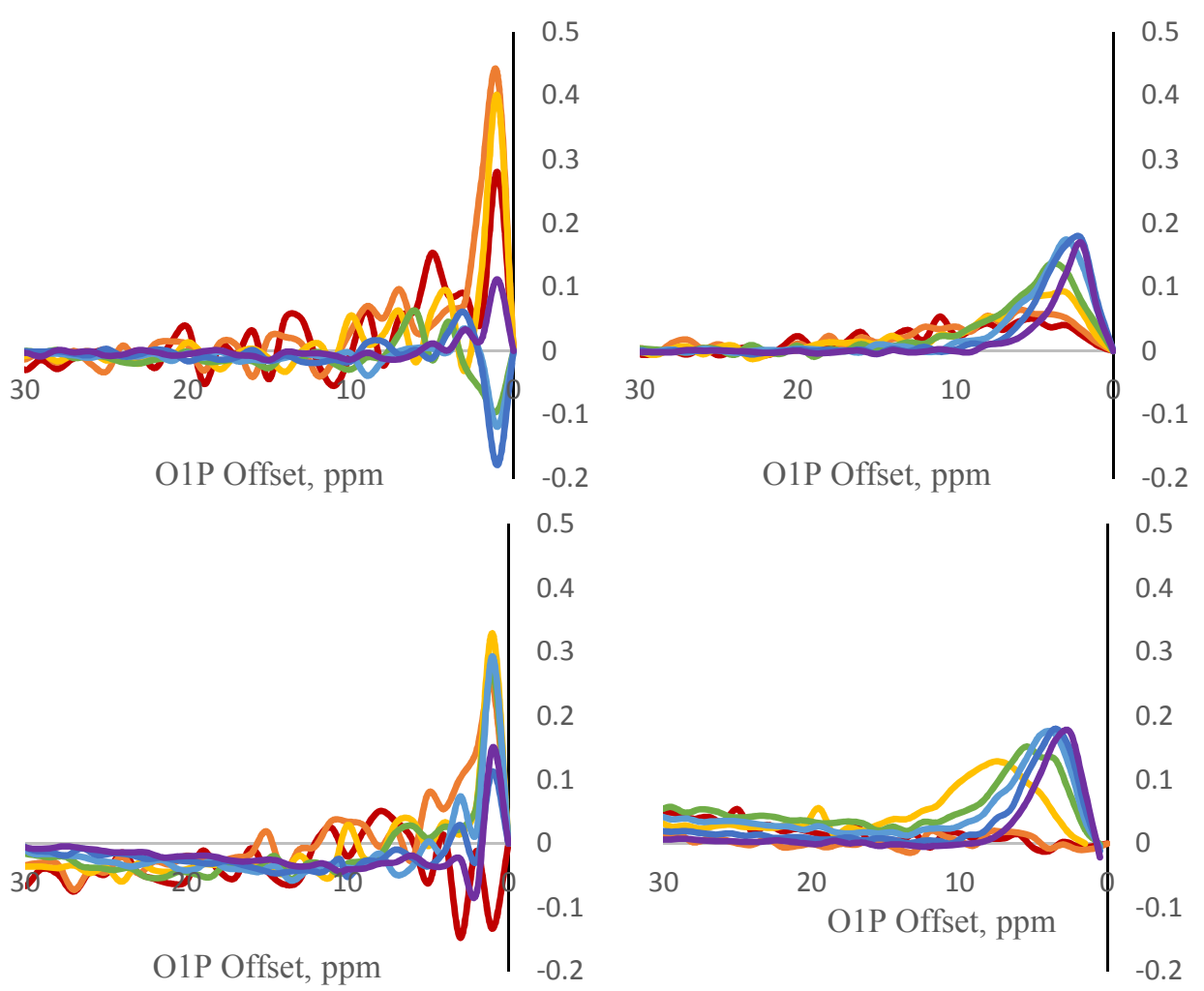

Figure 3.3.7. CEST asymmetric analysis. Top left: YDTPA ${ }^{-2} 200 \mathrm{~nm}$ LaPC. Top right: TmDTPA ${ }^{-2} 200 \mathrm{~nm}$ LaPC. Bottom left: YDTPA-2 $200 \mathrm{~nm}$ LaPC+C. Bottom right: TmDTPA ${ }^{-2} 200 \mathrm{~nm} \mathrm{LaPC}+\mathrm{C}$.

In spite of the CEST data not looking promising, a simulation was run to calculate the exchange rates in the system and the values suggested that the asymmetry in the recorded CEST spectra did not correspond to CEST from shifted water in the interior compartment. The LaPC vesicles have fast exchange since they are above the phase transition temperature at room temperature. Adding cholesterol does not slow the exchange rate down enough to have two compartments that are not well mixed. In order to further slow water exchange, a sample of $200 \mathrm{~nm}$ DPPC vesicles was made with $300 \mathrm{mM} \mathrm{TmDTPA}{ }^{-2}$ encapsulated in them.

\section{DPPC Vesicles}


The CEST spectra collected on the DPPC vesicles (Figure 3.3.8) looks similar to the $\mathrm{LaPC}$ and $\mathrm{LaPC}+\mathrm{C}$ CEST spectra (Figures 3.3.3, 3.3.4, 3.3.5, and 3.3.6), but instead of resembling the TmDTPA $^{-2}$ spectra, they look like a mixture between the TmDTPA ${ }^{-2}$ samples and the YDTPA ${ }^{-2}$ samples. There is broadening in the sample, and maybe some asymmetry, but the baseline is not clean and hard to interpret.

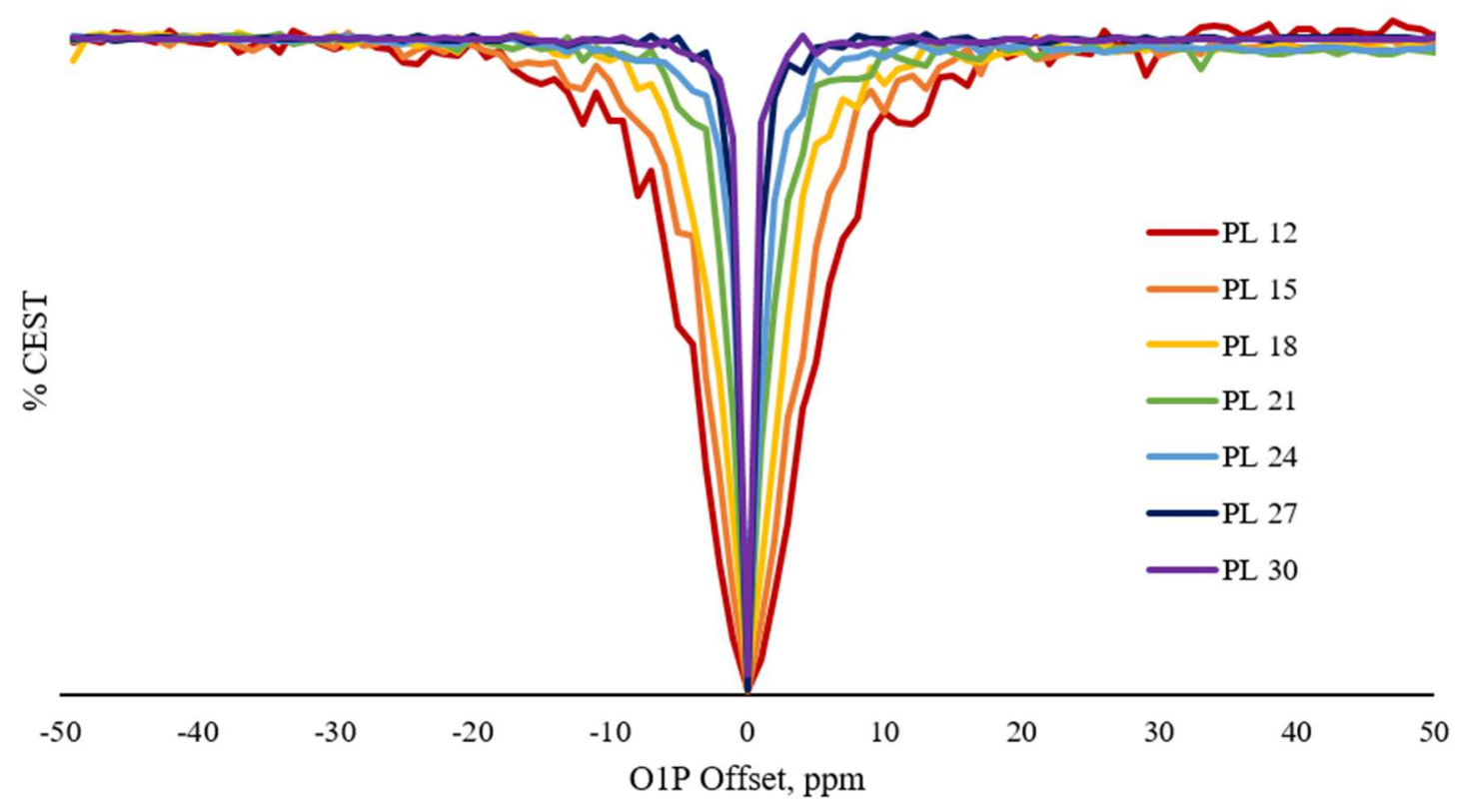

Figure 3.3.8. $300 \mathrm{mM} \mathrm{TmDTPA}{ }^{-2} 200 \mathrm{~nm}$ DPPC vesicle CEST spectra.

\subsection{Conclusions}

The LaPC vesicles studied have fast water exchange kinetics across the phospholipid bilayer, which makes them difficult to study by $\mathrm{T}_{1}$ and CEST analysis. The collection methods for both the $\mathrm{T}_{1}$ and CEST experiments need to be optimized for more studies. Also of interest would be using a different lipid system, like DPPC instead of LaPC. LaPC is above the phase transition temperature at room temperature while DPPC is above the phase transition temperature at room temperature. The DPPC vesicles were difficult to produce and were not pursued in these studies, but they should be, especially with the 
incorporation of cholesterol to help bring down the phase transition temperature to a more workable temperature instead of $43^{\circ} \mathrm{C}$. 


\section{CHAPTER 4. MATERIALS AND METHODS}

\subsection{Ln NB-DOTAM Project}

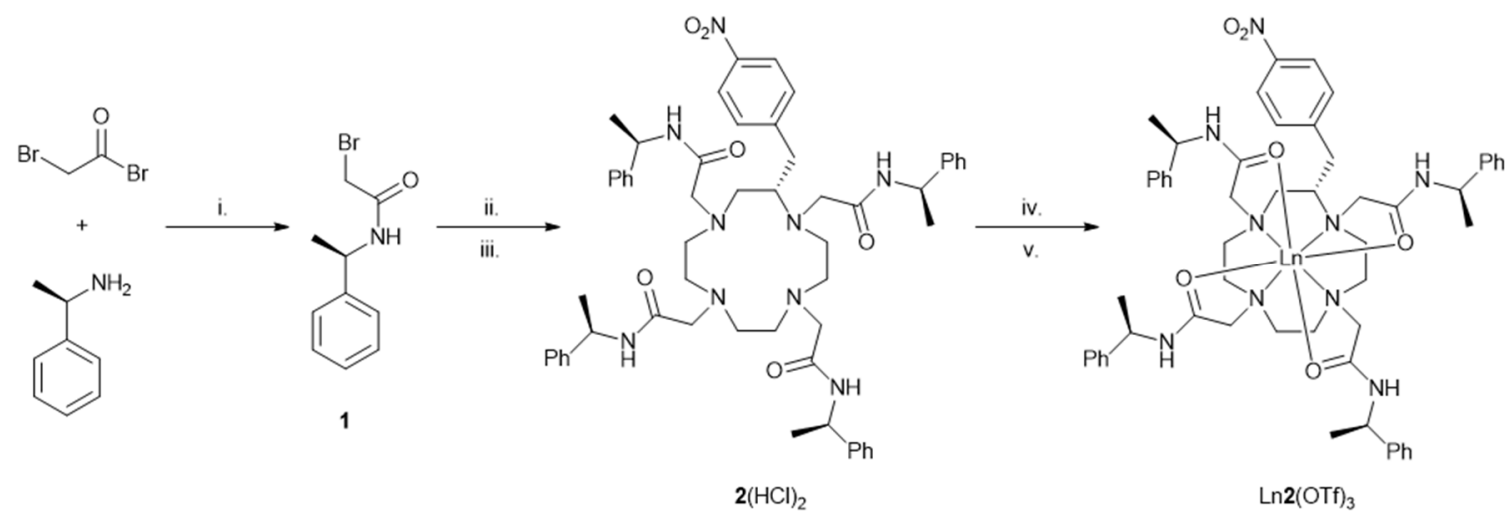

Figure 4.1.1. Synthetic route to Ln-NB-DOTAM chelates.

\section{General Remarks:}

All solvents and reagents were purchased from commercial sources and used as received unless otherwise stated. Both the $S$ - and $R$ - isomers of 2-bromo- $N$-(1phenylethyl)acetamide $\mathbf{1}$ were prepared according to previously described methods. HPLC purification was undertaken on a Waters $\delta$-Prep 150 HPLC system using a Phenomenex Luna C-18(2) reversed-phase $(50 \times 250 \mathrm{~mm})$ column. Elution was monitored by absorption at $270 \mathrm{~nm}$. All NMR spectra were recorded on a Bruker Advance IIa spectrometer operating at $400.13 \mathrm{MHz}\left({ }^{1} \mathrm{H}\right)$ and $100.61 \mathrm{MHz}\left({ }^{13} \mathrm{C}\right)$ using a $5 \mathrm{~mm}$ broadband probe with the temperature controlled using the installed variable-temperature controller BVT3200 with BCU-05 chiller. Samples for two-dimensional COSY spectroscopy were prepared in $\mathrm{CD}_{3} \mathrm{OD}$ (99.8 atom \%). Two-dimensional COSY spectra were acquired using $4096 \times 1024$ points using 16 transients per FID at $263 \mathrm{~K}$. Samples for CEST spectra were prepared in dry $\mathrm{CD}_{3} \mathrm{CN}$ (99.8 atom \%) and a known volume of $\mathrm{H}_{2} \mathrm{O}$ added. The concentrations of each sample was determined by comparison of the intensity of the ${ }^{19} \mathrm{~F}$ 
NMR signal with that of a $1.0 \mathrm{M}$ solution of $\mathrm{Cu}(\mathrm{OTf})_{2}$ placed in a concentric insert in the NMR tube. CEST spectra were acquired at $275 \mathrm{~K}$ acquired by measuring the water signal intensity as a function of presaturation offset, in $1 \mathrm{ppm}$ increments. The duration of the presaturation pulse was 10 s with a $\mathrm{B}_{1}$ power of $148,203,279,350$ and $446 \mathrm{~Hz}$.

$(1 R, 4 R, \quad 7 R, \quad 10 R)-\delta, \quad \delta^{\prime}, \quad \delta^{\prime \prime}, \quad \delta^{\prime \prime \prime}-$ Tetramethyl-[2-(S)-(p-nitrobenzyl)-1,4,7,10tetraazacyclo dodecane ]-1,4,7,10-tetra (2-phenylethyl)acetamide dihydrochloride salt (S-RRRR-2)

$R$-2-Bromo- $N$-(1-phenylethyl)acetamide 1 (497 mg, $2.05 \mathrm{mmol}) S$-2-(p-nitrobenzyl) cyclen $(150 \mathrm{mg}, 0.488 \mathrm{mmol})$ and potassium carbonate $(337 \mathrm{mg}, 2.44 \mathrm{mmol})$ were added to acetonitrile $(50 \mathrm{~mL})$. The resulting suspension was heated with stirring for 72 hours at $60{ }^{\circ} \mathrm{C}$. Following addition of more bromoacetamide $1(83 \mathrm{mg}, 0.34 \mathrm{mmol})$ the reaction was heated for a further 48 hours. After cooling to room temperature the solvents were removed under reduced pressure and the residue divided between water $(30 \mathrm{~mL})$ and dichloromethane $(200 \mathrm{~mL})$. After separation the aqueous phase was further extracted with dichloromethane $(2 \times 50 \mathrm{~mL})$, the organic phases were combined, dried $\left(\mathrm{Na}_{2} \mathrm{SO}_{4}\right)$ and the solvents removed under reduced pressure. The residue was taken up into a minimal amount of acetonitrile $(6 \mathrm{~mL})$ and purified by RP-HPLC employing a mobile phase of water $(0.37 \%$ $\mathrm{HCl}$ ) for 5 minutes followed by a linear gradient over 20 minutes to $60 \% \mathrm{MeCN}$ and $20 \%$ water $(0.37 \% \mathrm{HCl})$, after removal of the solvent by lyophilization the title compound was obtained as a colorless solid (1.4 g, 67\%).

$$
\mathrm{R}_{\mathrm{T}}=29.02 \mathrm{~min} ;{ }^{1} \mathrm{H} \mathrm{NMR}\left(\mathrm{CH}_{3} \mathrm{CN}, 400 \mathrm{MHz}\right) \delta 9.52\left(2 \mathrm{H}, \mathrm{d},{ }^{3} J_{\mathrm{H}-\mathrm{H}}=8 \mathrm{~Hz}, p-\mathrm{Ar}\right), 8.04
$$

$\left(2 \mathrm{H}, \mathrm{d},{ }^{3} J_{\mathrm{H}-\mathrm{H}}=8 \mathrm{~Hz}, p-\mathrm{Ar}\right), 8.49(2 \mathrm{H}, \mathrm{br}, \mathrm{CON} H), 8.38(2 \mathrm{H}, \mathrm{br}, \mathrm{CONH}), 7.4-6.9(20 \mathrm{H}, \mathrm{m}$, 
Ar), $4.91\left(4 \mathrm{H}, \mathrm{m}, \mathrm{CHCH}_{3}\right), 4.78\left(1 \mathrm{H}, \mathrm{dd}\left(\mathrm{aa}^{\prime}\right.\right.$ pattern $\left.),{ }^{3} \mathrm{~J}_{\mathrm{H}-\mathrm{H}}=7 \mathrm{~Hz}, \mathrm{CH}_{2} \mathrm{Ar}\right), 4.76(1 \mathrm{H}, \mathrm{dd}$ (aa' pattern), $\left.{ }^{3} \mathrm{~J}_{\mathrm{H}-\mathrm{H}}=7 \mathrm{~Hz}, \mathrm{CH}_{2} \mathrm{Ar}\right), 3.9-2.5\left(23 \mathrm{H}, \mathrm{m}\right.$ br, $\mathrm{NCH}_{2}$ ring and $\left.\mathrm{NCH}_{2} \mathrm{CO}\right), 1.36$ $\left(12 \mathrm{H}, \mathrm{m}, \mathrm{CH}_{3}\right) ;{ }^{13} \mathrm{C} \mathrm{NMR}\left(\mathrm{CH}_{3} \mathrm{CN}, 100 \mathrm{MHz}\right) \delta 20.9\left(\mathrm{CH}_{3}\right), 21.7\left(\mathrm{CH}_{3}\right), 22.0\left(\mathrm{CH}_{3}\right), 22.2$ $\left(\mathrm{CH}_{3}\right), 32.6\left(\mathrm{CH}_{2} \mathrm{Ar}\right), 48.6,48.8,48.9,49.0,49.3,49.5,49.69,50.2,51.1,51.6,52.8,53.3$, 53.4, 54.7, 56.5, 56.6, 123.2, 123.5, 125.7, 125.9, 126.3 - 126.9 (several, overlapping), 128.0 - 128.4 (several, overlapping), 130.2, 143.3, 143.6, 143.9, 144.6, 144.9, 146.4, 146.7, $161.8(\mathrm{C}=\mathrm{O}), 162.7(\mathrm{C}=\mathrm{O}), 167.9(\mathrm{C}=\mathrm{O}), 172.0(\mathrm{C}=\mathrm{O}) ; \mathrm{m} / \mathrm{z}(\mathrm{ESMS} \mathrm{EI}+)$ : $952(100 \%,[\mathrm{M}$ $\left.+\mathrm{H}]^{+}\right)$.

$(1 S, \quad 4 S, \quad 7 S, \quad 10 S)-\delta, \quad \delta^{\prime}, \quad \delta^{\prime \prime}, \quad \delta^{\prime \prime \prime}-$ Tetramethyl-[2-(S)-(p-nitrobenzyl)-1,4,7,10tetraazacyclo dodecane ]-1,4,7,10-tetra (2-phenylethyl)acetamide dihydrochloride salt (S-SSSS-2)

The title compound was prepared in an identical manner using $S$-2-bromo- $N$-(1phenylethyl)acetamide 1. After prep RP-HPLC the title compound was obtained as a colorless solid (0.8 g, $52 \%)$.

$\mathrm{R}_{\mathrm{T}}=29.84 \mathrm{~min} ;{ }^{1} \mathrm{H} \mathrm{NMR}\left(\mathrm{CD}_{3} \mathrm{CN}, 400 \mathrm{MHz}\right) 7.97\left(2 \mathrm{H}, \mathrm{d},{ }^{3} \mathrm{~J}_{\mathrm{H}-\mathrm{H}}=8 \mathrm{~Hz}, p-\mathrm{Ar}\right), 7.44$ $(20 \mathrm{H}, \mathrm{m}, \mathrm{Ar}), 7.31\left(2 \mathrm{H}, \mathrm{d},{ }^{3} J_{\mathrm{H}-\mathrm{H}}=8 \mathrm{~Hz}, p-\mathrm{Ar}\right), 7.02(4 \mathrm{H}, \mathrm{br}, \mathrm{CONH}), 4.91\left(4 \mathrm{H}, \mathrm{m}, \mathrm{CHCH}_{3}\right)$, 4.76 (2H, s br, $\left.\mathrm{CH}_{2} \mathrm{Ar}\right), 2.7-2.1\left(23 \mathrm{H}, \mathrm{m}\right.$ br, $\mathrm{NCH}_{2}$ ring and $\left.\mathrm{NCH}_{2} \mathrm{CO}\right), 1.31\left(12 \mathrm{H}, \mathrm{m}, \mathrm{CH}_{3}\right)$; ${ }^{13} \mathrm{C}$ NMR $\left(\mathrm{CH}_{3} \mathrm{CN}, 100 \mathrm{MHz}\right) \delta 21.2\left(\mathrm{CH}_{3}\right), 21.8\left(\mathrm{CH}_{3}\right), 22.1\left(\mathrm{CH}_{3}\right), 22.3\left(\mathrm{CH}_{3}\right), 36.4$ $\left(\underline{\mathrm{CH}}_{2} \mathrm{Ar}\right), 46.9,47.8,48.9,49.0,49.4,49.6,49.7,49.8,51.5,51.8,53.0,53.3,53.4,53.7$, 56.1, 57.0, 122.8, 123.1, 125.5 - 126.9 (several, overlapping), 128.4 - 128.7 (several, overlapping), 123.8, 131.4, 142.7, 143.0, 143.8, 144.9, 145.2, 146.7, 147.7, $162.0(\mathrm{C}=\mathrm{O})$, 
$162.5(\mathrm{C}=\mathrm{O}), 168.5(\mathrm{C}=\mathrm{O}), 171.4(\mathrm{C}=\mathrm{O}) ; \mathrm{m} / \mathrm{z}\left(\mathrm{ESMS} \mathrm{EI}^{+}\right)$: $952\left(100 \%,[\mathrm{M}+\mathrm{H}]^{+}\right), 974$ $\left(46 \%,[\mathrm{M}+\mathrm{Na}]^{+}\right)$.

\section{General method for the preparation of $\operatorname{Ln} 2(\mathrm{OTf})_{3}$ chelates.}

The corresponding lanthanide triflate $(73 \mu \mathrm{mol})$ was dissolved in $(4 \mathrm{~mL})$ and added to a solution of the ligand either S-RRRR- or S-SSSS-2 $(50 \mathrm{mg}, 49 \mu \mathrm{mol})$ in $50 / 50$ acetonitrile/water $(4 \mathrm{~mL})$. The resulting solution was heated with stirring for 24 hours at $60{ }^{\circ} \mathrm{C}$. The solution was filtered through a $0.45 \mu \mathrm{m}$ syringe filter and introduced directly to the HPLC for purification. Purification was undertaken using a mobile phase of water ( $0.1 \%$ TFA) for 5 minutes followed by a linear gradient to $10 \% \mathrm{MeCN}$ and $90 \%$ water ( $0.1 \%$ TFA) over 15 minutes. Each chelate was collected from a single major peak in the chromatogram and removal of the solvents by lyophilization afforded the chelate as the corresponding triflate salt as a colorless solid.

\section{Mass Spectrometry}

\section{$S$-RRRR-Ce2(OTf) 3}

HRMS (ESI-TOF) m/z: [M] ${ }^{3+}$ Calcd for $\mathrm{C}_{55} \mathrm{H}_{69} \mathrm{~N}_{9} \mathrm{CeO}_{6} 363.8142$; Found 363.8135; [M + OTf $]^{2+}$ Calcd for $\mathrm{C}_{56} \mathrm{H}_{69} \mathrm{~F}_{3} \mathrm{~N}_{9} \mathrm{CeO}_{9} \mathrm{~S}$ 620.1973; Found 622.1970; the appropriate $\mathrm{Ce}^{3+}$ isotope pattern was observed.

\section{$S$-RRRR-Pr2(OTf)3}

HRMS (ESI-TOF) m/z: [M] ${ }^{3+}$ Calcd for $\mathrm{C}_{55} \mathrm{H}_{69} \mathrm{~N}_{9} \mathrm{PrO}_{6}$ 364.1477; Found 364.1472; [M + OTf $]^{2+}$ Calcd for $\mathrm{C}_{56} \mathrm{H}_{69} \mathrm{~F}_{3} \mathrm{~N}_{9} \mathrm{PrO}_{9} \mathrm{~S}$ 620.6976; Found 620.6977; the appropriate $\operatorname{Pr}^{3+}$ isotope pattern was observed.

\section{$S-R R R R-N d 2(O T f)_{3}$}


HRMS (ESI-TOF) m/z: [M] ${ }^{3+}$ Calcd for $\mathrm{C}_{55} \mathrm{H}_{69} \mathrm{~N}_{9} \mathrm{NdO}_{6} 364.4816$; Found 364.4806; [M + OTf] ${ }^{2+}$ Calcd for $\mathrm{C}_{56} \mathrm{H}_{69} \mathrm{~F}_{3} \mathrm{~N}_{9} \mathrm{NdO}_{9} \mathrm{~S}$ 622.1996; Found 622.1986; the appropriate $\mathrm{Nd}^{3+}$ isotope pattern was observed.

\section{$S-R R R R-\operatorname{Sm} 2(\mathrm{OTf}) 3$}

HRMS (ESI-TOF) m/z: Calcd for $\mathrm{C}_{55} \mathrm{H}_{69} \mathrm{~N}_{9} \mathrm{SmO}_{6} 367.6189$; Found 367.6175; [M+OTf] ${ }^{2+}$ Calcd for $\mathrm{C}_{56} \mathrm{H}_{69} \mathrm{~F}_{3} \mathrm{~N}_{9} \mathrm{SmO}_{9} \mathrm{~S}$ 626.2044; Found 626.2028; the appropriate $\mathrm{Sm}^{3+}$ isotope pattern was observed.

\section{$S-R R R R-E u 2(O T f) 3$}

HRMS (ESI-TOF) m/z: [M] ${ }^{3+}$ Calcd for $\mathrm{C}_{55} \mathrm{H}_{69} \mathrm{~N}_{9} \mathrm{EuO}_{6} 368.1528$; Found 368.1538; [M + OTf] ${ }^{2+}$ Calcd for $\mathrm{C}_{56} \mathrm{H}_{69} \mathrm{~F}_{3} \mathrm{~N}_{9} \mathrm{EuO} 9 \mathrm{~S}$ 626.7052; Found 622.7074; the appropriate $\mathrm{Ce}^{3+}$ isotope pattern was observed.

\section{$S-R R R R-T b 2(O T f) 3$}

HRMS (ESI-TOF) m/z: [M] ${ }^{3+}$ Calcd for $\mathrm{C}_{55} \mathrm{H}_{69} \mathrm{~N}_{9} \mathrm{TbO}_{6} 370.1541$; Found 370.1533; [M + OTf] ${ }^{2+}$ Calcd for $\mathrm{C}_{56} \mathrm{H}_{69} \mathrm{~F}_{3} \mathrm{~N}_{9} \mathrm{TbO}_{9} \mathrm{~S}$ 629.7072; Found 629.7063 ; the appropriate $\mathrm{Tb}^{3+}$ isotope pattern was observed.

\section{$S-R R R R-D y 2(O T f) 3$}

HRMS (ESI-TOF) m/z: [M] ${ }^{3+}$ Calcd for $\mathrm{C}_{55} \mathrm{H}_{69} \mathrm{~N}_{9} \mathrm{DyO}_{6} 371.8221$; Found 371.8213; [M + OTf] ${ }^{2+}$ Calcd for $\mathrm{C}_{56} \mathrm{H}_{69} \mathrm{~F}_{3} \mathrm{~N}_{9}$ DyO9 ${ }_{9} \mathrm{~S}$ 632.2092; Found 632.2083; the appropriate $\mathrm{Dy}^{3+}$ isotope pattern was observed.

\section{$S$-RRRR-Er2(OTf)3}


HRMS (ESI-TOF) m/z: [M] ${ }^{3+}$ Calcd for $\mathrm{C}_{55} \mathrm{H}_{69} \mathrm{~N}_{9} \mathrm{ErO}_{6}$ 372.4891; Found 372.4881; [M + OTf $]^{2+}$ Calcd for $\mathrm{C}_{56} \mathrm{H}_{69} \mathrm{~F}_{3} \mathrm{~N}_{9} \mathrm{ErO}_{9} \mathrm{~S}$ 634.2108; Found 634.2098; the appropriate $\mathrm{Er}^{3+}$ isotope pattern was observed.

\section{$S-R R R R-Y b 2(O T f) 3$}

HRMS (ESI-TOF) m/z: [M] ${ }^{3+}$ Calcd for $\mathrm{C}_{55} \mathrm{H}_{69} \mathrm{~N}_{9} \mathrm{YbO}_{6} 375.1586$; Found 375.1589; [M + OTf] ${ }^{2+}$ Calcd for $\mathrm{C}_{56} \mathrm{H}_{69} \mathrm{~F}_{3} \mathrm{~N}_{9} \mathrm{YbO}_{9} \mathrm{~S}$ 637.2140; Found 637.2162; the appropriate $\mathrm{Yb}^{3+}$ isotope pattern was observed.

\section{S-SSSS-Ce2(OTf)3}

HRMS (ESI-TOF) m/z: [M] ${ }^{3+}$ Calcd for $\mathrm{C}_{55} \mathrm{H}_{69} \mathrm{~N}_{9} \mathrm{CeO}_{6}$ 363.8142; Found 363.8133; [M + OTf] ${ }^{2+}$ Calcd for $\mathrm{C}_{56} \mathrm{H}_{69} \mathrm{~F}_{3} \mathrm{~N}_{9} \mathrm{CeO}_{9} \mathrm{~S}$ 620.1973; Found 620.1967; the appropriate $\mathrm{Ce}^{3+}$ isotope pattern was observed.

\section{$S$-SSSS-Pr2(OTf)3}

HRMS (ESI-TOF) m/z: [M] ${ }^{3+}$ Calcd for $\mathrm{C}_{55} \mathrm{H}_{69} \mathrm{~N}_{9} \mathrm{PrO}_{6}$ 364.1477; Found 364.1473; [M + OTf $]^{2+}$ Calcd for $\mathrm{C}_{56} \mathrm{H}_{69} \mathrm{~F}_{3} \mathrm{~N}_{9} \mathrm{PrO}_{9} \mathrm{~S}$ 620.6976; Found 620.6976; the appropriate $\operatorname{Pr}^{3+}$ isotope pattern was observed.

\section{S-SSSS-Eu2(OTf)3}

HRMS (ESI-TOF) m/z: [M] ${ }^{3+}$ Calcd for $\mathrm{C}_{55} \mathrm{H}_{69} \mathrm{~N}_{9} \mathrm{EuO}_{6}$ 368.1528; Found 368.1537; [M + OTf $]^{2+}$ Calcd for $\mathrm{C}_{56} \mathrm{H}_{69} \mathrm{~F}_{3} \mathrm{~N}_{9} \mathrm{EuO} 9 \mathrm{~S}$ 626.7052; Found 626.7072; the appropriate $\mathrm{Eu}^{3+}$ isotope pattern was observed.

\section{S-SSSS-Tb2(OTf)3}


HRMS (ESI-TOF) m/z: [M] ${ }^{3+}$ Calcd for $\mathrm{C}_{55} \mathrm{H}_{69} \mathrm{~N}_{9} \mathrm{TbO}_{6} 370.1541$; Found 370.1532; [M + OTf $]^{2+}$ Calcd for $\mathrm{C}_{56} \mathrm{H}_{69} \mathrm{~F}_{3} \mathrm{~N}_{9} \mathrm{TbO}_{9} \mathrm{~S}$ 629.7072; Found 629.7062; the appropriate $\mathrm{Tb}^{3+}$ isotope pattern was observed.

\section{$S$-SSSS-Dy2(OTf) 3}

HRMS (ESI-TOF) m/z: [M] ${ }^{3+}$ Calcd for $\mathrm{C}_{55} \mathrm{H}_{69} \mathrm{~N}_{9} \mathrm{DyO}_{6}$ 371.8221; Found 371.8208; [M + OTf] ${ }^{2+}$ Calcd for $\mathrm{C}_{56} \mathrm{H}_{69} \mathrm{~F}_{3} \mathrm{~N}_{9} \mathrm{DyO}_{9} \mathrm{~S}$ 632.2092; Found 632.2075; the appropriate $\mathrm{Dy}^{3+}$ isotope pattern was observed.

\section{S-SSSS-Ho2(OTf)3}

HRMS (ESI-TOF) m/z: [M] ${ }^{3+}$ Calcd for $\mathrm{C}_{55} \mathrm{H}_{69} \mathrm{~N}_{9} \mathrm{HoO}_{6} 372.1558$; Found 372.1547; [M + OTf] ${ }^{2+}$ Calcd for $\mathrm{C}_{56} \mathrm{H}_{69} \mathrm{~F}_{3} \mathrm{~N}_{9} \mathrm{HoO}_{9} \mathrm{~S}$ 632.7097; Found 632.7086; the appropriate $\mathrm{Ho}^{3+}$ isotope pattern was observed.

\section{S-SSSS-Er2(OTf) 3}

HRMS (ESI-TOF) m/z: [M] ${ }^{3+}$ Calcd for $\mathrm{C}_{55} \mathrm{H}_{69} \mathrm{~N}_{9} \mathrm{ErO}_{6}$ 372.4891; Found 372.4886; [M + OTf] ${ }^{2+}$ Calcd for $\mathrm{C}_{56} \mathrm{H}_{69} \mathrm{~F}_{3} \mathrm{~N}_{9} \mathrm{ErO} \mathrm{O}_{9} \mathrm{~S}$ 634.2108; Found 634.2001; the appropriate $\mathrm{Er}^{3+}$ isotope pattern was observed.

\section{S-SSSS-Yb2(OTf) 3}

HRMS (ESI-TOF) m/z: [M] ${ }^{3+}$ Calcd for $\mathrm{C}_{55} \mathrm{H}_{69} \mathrm{~N}_{9} \mathrm{YbO}_{6}$ 375.1586; Found 375.1599; [M + OTf] ${ }^{2+}$ Calcd for $\mathrm{C}_{56} \mathrm{H}_{69} \mathrm{~F}_{3} \mathrm{~N}_{9} \mathrm{YbO}_{9} \mathrm{~S}$ 637.2140; Found 622.2162; the appropriate $\mathrm{Yb}^{3+}$ isotope pattern was observed. 


\subsection{Vesicle Project}

\section{Vesicle Synthesis}

$0.05 \mathrm{~g} \mathrm{~L}-\alpha-$ phosphatidylcholine was weighed into a round bottom flask. Chloroform was added to dissolve the lipid and then removed under vacuum. The lipid was then suspended in the desired buffer system, agitated through swirling, stirring, and rotating until the thin film of lipid was removed from the wall of the round bottom flask. The lipid was then extruded through the Lipex $10 \mathrm{~mL}$ water jacketed extruder (TRANSFERRA Nanosciences Inc) through polycarbonate membranes with pores of the desired sizes a total of 10 times. The polycarbonate membranes were removed and placed at any point in the extrusion process if the synthesis was difficult and lipid was falling out of solution and blocking the passage of the vesicle mixture through the filter. After 10 passes through the desired filter size, the samples were analyzed by DLS for sizing.

If the chelate being studied was inside the vesicles, it was in the buffer solution used to make the vesicles after solvent was removed under vacuum. Once the vesicles were sized, $1 \mathrm{~mL}$ of the sample was placed into a Float-a-lyzer dialysis membrane and was dialyzed against bath buffer to remove the excess chelate. If the chelate being studied was outside the vesicles, it was added to a small sample of prepared vesicles $(1 \mathrm{~mL})$.

\section{Collecting the Data}

The $\mathrm{T}_{1}$ inversion recovery curve experiment requires picking $\tau$ values that allow some of the sample signal to relax back to the z-axis and some of it to not relax back to the zaxis. Picking $\tau$ values appropriate for the sample in question is generally done on a "Is it expected to have a short or long $\mathrm{T}_{1}$ ?" basis. However, in the case of vesicles with 
GdDTPA $^{-2}$, two $T_{1}$ values are present and may require equal consideration while picking $\tau$ values for the experiment. As an example, the $5 \mathrm{mM} \mathrm{GdDPTA}^{-2}$ sample was run with the $5 \mathrm{mM} \mathrm{GdDTPA}^{-2}$ VD list, which has shorter $\tau$ values $(5.2 \mathrm{~ms}$ to $600 \mathrm{~ms})$ than all of the other sample VD lists, and the $1 \mathrm{mM}$ GdDTPA-2 sample was run with the $1 \mathrm{mM} \mathrm{GdDTPA}{ }^{-}$ ${ }^{2}$ VD list, which has longer $\tau$ values ( $26 \mathrm{~ms}$ to $3 \mathrm{~s}$ ) than the other sample VD lists. In the case of the $1 \mathrm{mM} \mathrm{GdDTPA}^{-2}$ vesicle sample, the amount of signal belonging to encapsulated water (the longer $T_{1}$ value) wouldn't really be cut down that much due to the $1 \mathrm{mM} \mathrm{GdDTPA}^{-2}$ using longer $\tau$ values, where the amount of signal belonging to encapsulated water would be cut off quite a bit since the longest it waits for is $600 \mathrm{~ms}$. By using the concentration dependent VD lists, the samples were essentially being $\mathrm{T}_{1}$ weighted. In order to see if this was occurring, a concentration independent VD list was used, with $\tau$ values from $5 \mathrm{~ms}$ to $15 \mathrm{~s}$. This data collection method was employed for several different samples after the idea was brough up, but it wasn't until later in the experiments, meaning there is no normal system to compare to. Therefore, the concentration independent VD list used is compared to the same sample run with the concentration dependent VD list.

\section{Processing the Data}

Some data, when processed using the "original (O) method" of analysis, did not produce recovery curves that could be considered useful. In particular, $0 \mathrm{mM} \mathrm{GdDTPA}{ }^{-2}$ samples (samples of just buffer and vesicles) and $1 \mathrm{mM} \mathrm{GdDTPA}^{-2}$ samples provided difficult recovery curves. After attempting to make sure the relaxation delays and VD lists were appropriate, Dr. Peyton was kind enough to help with the data analysis portion done on the spectrometer computer. Dr. Peyton first phased and then baseline corrected the last 
spectrum of each experimental run and applied the analysis to all of the spectra collected in the experiment. The $T_{1}$ analysis was followed, but instead of an integration assignment for the program to follow, he manually picked the peak to follow. After his changes to the method, difficult samples that produced messy or incomplete recovery curves suddenly had near perfect recovery curves. Since the analysis method was suggested late in the data collection process, all of the previously analyzed data using the original method were reanalyzed using the "professional (P) method". The analysis using excel was the same no matter the type of analysis that was performed on the spectrometer computer.

\section{Monoexponential Fitting}

The intensity values from the text file were normalized using the following equation:

$$
\text { Monoexponential Experimental Data Point }=\frac{I(\tau)}{I(\infty)}
$$

Where $\mathrm{I}(\tau)$ is the intensity at that $\tau$ value and $\mathrm{I}(\infty)$ is the intensity at an assumed $\infty \tau$ value where the spins have relaxed completely. For the $600 \mathrm{MHz}$ data, the I( $\infty)$ value was taken to be the highest intensity point in the experimental data, which was at the end of the experiment run with the longest $\tau$ value.

The recovery curve from the experimental intensity values was compared to a calculated curve which was produced from a population size, $\mathrm{M}_{0}$, a relaxivity value, $\mathrm{R}_{1}$, and the time point, $\tau$, and using the following equation:

$$
\text { Monoexponential Calculated Data Point }=M_{0}-2 M_{0} * e^{\left(-\tau * R_{1}\right)}
$$

The values of $\mathrm{M}_{0}$ and $\mathrm{R}_{1}$ were allowed to float in the calculation so that they would be representative of the species being measured. 
The two sets (the experimental and calculated) were analyzed using the following equation in excel:

$=S Q R T\left(\frac{S U M X M Y 2(\text { range of experimental values, range of calculated values })}{S U M S Q(\text { range of experimental values })}\right)$

The resulting value was minimized using the data solver function in excel, with the values of $M_{0}$ and $R_{1}$ being able to change to make the best match of the calculated values to the experimental values. Once the fit between the two sets of data was minimized, the $\mathrm{R}_{1}$ and $\mathrm{M}_{0}$ values were used to assess the $T_{1}$ of the sample and the population size, respectively.

\section{Biexponential Fitting}

The intensity values from the text file were manipulated using the following equation from a paper where biexponential fitting was performed:

$$
\text { Biexponential Experimental Data Point }=\frac{(I(\infty)-I(\tau))}{2 * I(\infty)}
$$

Where $\mathrm{I}(\infty)$ is the intensity at an assumed $\infty \tau$ value where the spins have relaxed completely (the highest intensity point in the experimental data at the end of the experiment run with the longest $\tau$ value ) and $\mathrm{I}(\tau)$ is the intensity at that $\tau$ value.

The experimental intensity values were compared to a calculated curve which was produced from a small population size, $\mathrm{M}_{\mathrm{S}}$, and a small population relaxivity value, $\mathrm{R}_{\mathrm{S} 1}$, a large population size, $\mathrm{M}_{\mathrm{L}}$, and a large population relaxivity value, $\mathrm{R}_{\mathrm{L} 1}$, and the time point, $\tau$, and using the following equation:

$$
\text { Biexponential Calculated Data Point }=M_{S} * e^{\left(-\tau * R_{S 1}\right)}+M_{L} * e^{\left(-\tau * R_{L 1}\right)}
$$


The values of $M_{S}, M_{L}, R_{S 1}$, and $R_{L 1}$ were allowed to float in the calculation so that they would be representative of the species being measured.

The two sets (the experimental and calculated) were analyzed using the following equation in excel:

$=S Q R T\left(\frac{S U M X M Y 2(\text { range of experimental values, range of calculated values })}{S U M S Q(\text { range of experimental values })}\right)$

The resulting value was minimized using the data solver function in excel, with the values of $M_{S}, M_{L}, R_{S 1}$, and $R_{L 1}$ being able to change to make the best match of the calculated values to the experimental values. Once the fit between the two sets of data was minimized, the $M_{S}, M_{L}, R_{S 1}$, and $R_{L 1}$ values were used to assess the $T_{1}$ values of the two species in the sample and the population sizes of each.

\section{CEST Experiments}

CEST spectra were acquired at $25^{\circ} \mathrm{C}$ acquired by measuring the water signal intensity as a function of presaturation offset, in $1 \mathrm{ppm}$ increments. The duration of the presaturation pulse was 10 s with a $\mathrm{B}_{1}$ power of $148,203,279,350$ and $446 \mathrm{~Hz}$. 


\section{References}

(1) Rontgen, W. C.: On a New Kind of Rays. Science 1896, 3, 227-31.

(2) Cho, Z. H.; Tsai, C. M.; Wilson, G.: Study of contrast and modulation mechanisms in X-ray/photon transverse axial transmission tomography. Physics in medicine and biology 1975, 20, 879-89.

(3) Hande, M. P.; Azizova, T. V.; Geard, C. R.; Burak, L. E.; Mitchell, C. R.; Khokhryakov, V. F.; Vasilenko, E. K.; Brenner, D. J.: Past exposure to densely ionizing radiation leaves a unique permanent signature in the genome. American journal of human genetics 2003, 72, 1162-70.

(4) Zhou, H.; Ivanov, V. N.; Gillespie, J.; Geard, C. R.; Amundson, S. A.; Brenner, D. J.; Yu, Z.; Lieberman, H. B.; Hei, T. K.: Mechanism of radiation-induced bystander effect: role of the cyclooxygenase-2 signaling pathway. Proceedings of the National Academy of Sciences of the United States of America 2005, 102, 14641-6.

(5) Hei, T. K.; Zhou, H.; Ivanov, V. N.; Hong, M.; Lieberman, H. B.; Brenner, D. J.; Amundson, S. A.; Geard, C. R.: Mechanism of radiation-induced bystander effects: a unifying model. The Journal of pharmacy and pharmacology 2008, 60, 943-50.

(6) Shuryak, I.; Brenner, D. J.; Ullrich, R. L.: Radiation-induced carcinogenesis: mechanistically based differences between gamma-rays and neutrons, and interactions with DMBA. PloS one 2011, 6, e28559.

(7) Hall, E. J.; Brenner, D. J.: Cancer risks from diagnostic radiology: the impact of new epidemiological data. The British journal of radiology 2012, 85, e1316-7.

(8) Brenner, D. J.; Hall, E. J.: Cancer risks from CT scans: now we have data, what next? Radiology 2012, 265, 330-1.

(9) Brenner, D. J.: What we know and what we don't know about cancer risks associated with radiation doses from radiological imaging. The British journal of radiology 2014, 87, 20130629.

(10) Shuryak, I.; Fornace, A. J., Jr.; Datta, K.; Suman, S.; Kumar, S.; Sachs, R. K.; Brenner, D. J.: Scaling Human Cancer Risks from Low LET to High LET when DoseEffect Relationships are Complex. Radiation research 2017, 187, 476-482.

(11) Dussik, K. T.; Dussik, F.; Wyt, L.: [Not Available]. Wien Med Wochenschr 1947, $97,425-9$.

(12) Wild, J. J.: The use of ultrasonic pulses for the measurement of biologic tissues and the detection of tissue density changes. Surgery 1950, 27, 183-8.

(13) Carovac, A.; Smajlovic, F.; Junuzovic, D.: Application of ultrasound in medicine. Acta informatica medica : AIM : journal of the Society for Medical Informatics of Bosnia \& Herzegovina : casopis Drustva za medicinsku informatiku BiH 2011, 19, 16871. $161,609$.

(14) Becquerel, J.; Crowther, J. A.: Discovery of radioactivity. Nature 1948,

(15) Hevesy, G.: The Absorption and Translocation of Lead by Plants: A Contribution to the Application of the Method of Radioactive Indicators in the Investigation of the Change of Substance in Plants. The Biochemical journal 1923, 17, 43945 . 
(16) Hahn, L. A.; Hevesy, G. C.; Lundsgaard, E. C.: The circulation of phosphorus in the body revealed by application of radioactive phosphorus as indicator. The Biochemical journal 1937, 31, 1705-9.

(17) Lassen, N. A.; Ingvar, D. H.; Skinhoj, E.: Brain function and blood flow. Scientific American 1978, 239, 62-71.

(18) Shukla, A. K.; Kumar, U.: Positron emission tomography: An overview. Journal of medical physics 2006, 31, 13-21.

(19) Hansen, H.; Loft, A.; Berthelsen, A. K.; Lassen, S.; Hogdall, C.; Engelholm, S. A.: Hilgh Sensitivity and Specificity of PET/CT and Laparoscopic Diagnostic Lymph Node Excision for Lymph Node Metastases in Cervical Cancer Patients. Practical radiation oncology 2013, 3 , S10.

(20) Christensen, T. E.; Bang, L. E.; Holmvang, L.; Ghotbi, A. A.; Lassen, M. L.; Andersen, F.; Ihlemann, N.; Andersson, H.; Grande, P.; Kjaer, A.; Hasbak, P.: Cardiac (9)(9)mTc sestamibi SPECT and (1)(8)F FDG PET as viability markers in Takotsubo cardiomyopathy. The international journal of cardiovascular imaging 2014, 30, 1407-16.

(21) Singh, N.; Kumar, R.; Malhotra, A.; Bhalla, A. S.; Kumar, U.; Sood, R.: Diagnostic utility of fluorodeoxyglucose positron emission tomography/computed tomography in pyrexia of unknown origin. Indian journal of nuclear medicine: IJNM : the official journal of the Society of Nuclear Medicine, India 2015, 30, 204-12.

(22) Sarasamkan, J.; Fischer, S.; Deuther-Conrad, W.; Ludwig, F. A.; Scheunemann, M.; Arunrungvichian, K.; Vajragupta, O.; Brust, P.: Radiosynthesis of (S)[18F]T1: The first PET radioligand for molecular imaging of alpha3beta4 nicotinic acetylcholine receptors. Applied radiation and isotopes : including data, instrumentation and methods for use in agriculture, industry and medicine 2017, 124, 106-113.

(23) Bellani, G.; Rouby, J. J.; Constantin, J. M.; Pesenti, A.: Looking closer at acute respiratory distress syndrome: the role of advanced imaging techniques. Current opinion in critical care 2017, 23, 30-37.

(24) Singh, D.; Chopra, A.; Ravina, M.; Kongara, S.; Bhatia, E.; Kumar, N.; Gupta, S.; Yadav, S.; Dabadghao, P.; Yadav, R.; Dube, V.; Kumar, U.; Dixit, M.; Gambhir, S.: Oncogenic osteomalacia: role of Ga-68 DOTANOC PET/CT scan in identifying the culprit lesion and its management. The British journal of radiology 2017, 90, 20160811.

(25) Fischer, B. M.; Mortensen, J.; Langer, S. W.; Loft, A.; Berthelsen, A. K.; Daugaard, G.; Lassen, U.; Hansen, H. H.: PET/CT imaging in response evaluation of patients with small cell lung cancer. Lung Cancer 2006, 54, 41-9.

(26) Wang, X.; Zhan, Y.; Zhao, L.; Alvarez, J.; Chaudhary, I.; Zhou, B. B.; Abraham, R. T.; Feuerstein, G. Z.: Multimodal biomarker investigation on efficacy and mechanism of action for the mammalian target of rapamycin inhibitor, temsirolimus, in a preclinical mammary carcinoma OncoMouse model: a translational medicine study in support for early clinical development. The Journal of pharmacology and experimental therapeutics 2011, 339, 421-9.

(27) Ireland, R. H.; Tahir, B. A.; Wild, J. M.; Lee, C. E.; Hatton, M. Q.: Functional Image-guided Radiotherapy Planning for Normal Lung Avoidance. Clin Oncol (R Coll Radiol) 2016, 28, 695-707. 
(28) Lemke, H.; de Castro, A. G.; Schlattmann, P.; Heuser, I.; Neu, P.: Cerebrovascular reactivity over time-course - from major depressive episode to remission. Journal of psychiatric research 2010, 44, 132-6.

(29) Slifstein, M.; Abi-Dargham, A.: Recent Developments in Molecular Brain Imaging of Neuropsychiatric Disorders. Seminars in nuclear medicine 2017, 47, 54-63.

(30) Norbak-Emig, H.; Pinborg, L. H.; Raghava, J. M.; Svarer, C.; Baare, W. F. C.; Allerup, P.; Friberg, L.; Rostrup, E.; Glenthoj, B.; Ebdrup, B. H.: Extrastriatal dopamine D2/3 receptors and cortical grey matter volumes in antipsychotic-naive schizophrenia patients before and after initial antipsychotic treatment. The world journal of biological psychiatry : the official journal of the World Federation of Societies of Biological Psychiatry 2016, 1-11.

(31) Ejareh Dar, M.; Kanaan, R. A.: Uncovering the etiology of conversion disorder: insights from functional neuroimaging. Neuropsychiatric disease and treatment 2016, 12, 143-53.

(32) Su, C.; Hu, R.; Gu, Y.; Han, R.; Yan, X.; Liao, Q.; Huang, D.: The Nuclear Molecular Imaging of Protein Brain Receptors in Chronic Pain. Current protein \& peptide science 2016, 17, 559-69.

(33) Hammad, B.; Evans, N. R.; Rudd, J. H. F.; Tawakol, A.: Molecular imaging of atherosclerosis with integrated PET imaging. Journal of nuclear cardiology : official publication of the American Society of Nuclear Cardiology 2017, 24, 938-943.

(34) Ehlgen, A.; Bylock, A.; Kreuzer, J.; Koslowski, M.; Gantner, F.; Niessen, H. G.: Clinical imaging in anti-atherosclerosis drug development. Drug discovery today 2015, 20, 1317-27.

(35) Kumar, A.; Williams, M. T.; Chugani, H. T.: Evaluation of basal ganglia and thalamic inflammation in children with pediatric autoimmune neuropsychiatric disorders associated with streptococcal infection and tourette syndrome: a positron emission tomographic (PET) study using 11C-[R]-PK11195. Journal of child neurology 2015, 30, 749-56.

(36) Garcia, G. J.; Chagas, M. H.; Silva, C. H.; Machado-de-Sousa, J. P.; Crippa, J. A.; Hallak, J. E.: Structural and functional neuroimaging findings associated with the use of clozapine in schizophrenia: a systematic review. Rev Bras Psiquiatr 2015, 37, 71-9.

(37) Ferreira, L. K.; Tamashiro-Duran, J. H.; Squarzoni, P.; Duran, F. L.; Alves, T. C.; Buchpiguel, C. A.; Busatto, G. F.: The link between cardiovascular risk, Alzheimer's disease, and mild cognitive impairment: support from recent functional neuroimaging studies. Rev Bras Psiquiatr 2014, 36, 344-57.

(38) Topakian, R.; Pischinger, B.; Stieglbauer, K.; Pichler, R.: Rare clinical findings in a patient with sporadic hemiplegic migraine: FDG-PET provides diminished brain metabolism at 10-year follow-up. Cephalalgia : an international journal of headache 2014, 34, 392-6.

(39) Poels, E. M.; Kegeles, L. S.; Kantrowitz, J. T.; Slifstein, M.; Javitt, D. C.; Lieberman, J. A.; Abi-Dargham, A.; Girgis, R. R.: Imaging glutamate in schizophrenia: review of findings and implications for drug discovery. Molecular psychiatry 2014, 19, 209. 
(40) Heiss, W. D.: Radionuclide imaging in ischemic stroke. Journal of nuclear medicine : official publication, Society of Nuclear Medicine 2014, 55, 1831-41.

(41) Kim, J.; Choi, K. H.; Song, H. C.; Kim, J. T.; Park, M. S.; Cho, K. H.: 18FFDG PET/CT imaging factors that predict ischaemic stroke in cancer patients. European journal of nuclear medicine and molecular imaging 2016, 43, 2228-2235.

(42) Noto, B.; Vrachimis, A.; Schafers, M.; Stegger, L.; Rahbar, K.: Subacute Stroke Mimicking Cerebral Metastasis in 68Ga-PSMA-HBED-CC PET/CT. Clinical nuclear medicine 2016, 41, e449-51.

(43) Werner, P.; Saur, D.; Mildner, T.; Moller, H.; Classen, J.; Sabri, O.; Hoffmann, K. T.; Barthel, H.: Combined PET/MRI: Multimodality insights into acute stroke hemodynamics. Neurology 2016, 86, 1926-7.

(44) Nieto, E.; Delgado, M.; Sobrado, M.; de Ceballos, M. L.; Alajarin, R.; Garcia-Garcia, L.; Kelly, J.; Lizasoain, I.; Pozo, M. A.; Alvarez-Builla, J.: Preliminary research on 1-(4-bromo-2-nitroimidazol-1-yl)-3-[(18)F]fluoropropan-2-ol as a novel brain hypoxia PET tracer in a rodent model of stroke. European journal of medicinal chemistry 2015, 101, 604-15.

(45) Werner, P.; Saur, D.; Zeisig, V.; Ettrich, B.; Patt, M.; Sattler, B.; Jochimsen, T.; Lobsien, D.; Meyer, P. M.; Bergh, F. T.; Dreyer, A.; Boltze, J.; Classen, J.; Fritzsch, D.; Hoffmann, K. T.; Sabri, O.; Barthel, H.: Simultaneous PET/MRI in stroke: a case series. Journal of cerebral blood flow and metabolism : official journal of the International Society of Cerebral Blood Flow and Metabolism 2015, 35, 1421-5.

(46) Ponrartana, S.; Hu, H. H.; Gilsanz, V.: On the relevance of brown adipose tissue in children. Annals of the New York Academy of Sciences 2013, 1302, 24-9.

(47) Gilsanz, V.; Hu, H. H.; Kajimura, S.: Relevance of brown adipose tissue in infancy and adolescence. Pediatric research 2013, 73, 3-9.

(48) Angelidis, G.; Giamouzis, G.; Karagiannis, G.; Butler, J.; Tsougos, I.; Valotassiou, V.; Giannakoulas, G.; Dimakopoulos, N.; Xanthopoulos, A.; Skoularigis, J.; Triposkiadis, F.; Georgoulias, P.: SPECT and PET in ischemic heart failure. Heart failure reviews 2017, 22, 243-261.

(49) Gholoobi, A.; Ayati, N.; Baghyari, A.; Mouhebati, M.; Atar, B.; Dabbagh Kakhki, V. R.: Relationship between gated myocardial perfusion SPECT findings and hemodynamic, electrocardiographic, and heart rate changes after Dipyridamole infusion. The international journal of cardiovascular imaging 2017, 33, 951-956.

(50) Srivatsava, M. K.; Indirani, M.; Sathyamurthy, I.; Sengottuvelu, G.; Jain, A. S.; Shelley, S.: Role of PET-CT in the assessment of myocardial viability in patients with left ventricular dysfunction. Indian heart journal 2016, 68, 693-699.

(51) Pottker, B.; Stober, F.; Hummel, R.; Angenstein, F.; Radyushkin, K.; Goldschmidt, J.; Schafer, M. K. E.: Traumatic brain injury causes long-term behavioral changes related to region-specific increases of cerebral blood flow. Brain structure \& function 2017.

(52) Shin, S. S.; Bales, J. W.; Edward Dixon, C.; Hwang, M.: Structural imaging of mild traumatic brain injury may not be enough: overview of functional and metabolic imaging of mild traumatic brain injury. Brain imaging and behavior 2017, 11, 591-610. 
(53) Molina-Vicenty, I. L.; Santiago-Sanchez, M.; Velez-Miro, I.; MottaValencia, K.: Impact of Single-Photon Emission Computed Tomography/Computed Tomography (SPECT/CT) and Positron Emission Tomography/Computed Tomography (PET/CT) in the Diagnosis of Traumatic Brain Injury (TBI): Case Report. Puerto Rico health sciences journal 2016, 35, 170-2.

(54) Hadanny, A.; Golan, H.; Fishlev, G.; Bechor, Y.; Volkov, O.; Suzin, G.; Ben-Jacob, E.; Efrati, S.: Hyperbaric oxygen can induce neuroplasticity and improve cognitive functions of patients suffering from anoxic brain damage. Restorative neurology and neuroscience 2015, 33, 471-86.

(55) Cierniak, R.: Some Words About the History of Computed Tomography. In X-Ray Computed Tomography in Biomedical Engineering; Springer, 2011; pp 7-19.

(56) Rabi, I. I.; Kellogg, J. M. B.; Zacharias, J. R.: The magnetic moment of the proton. Physical Review 1934, 46, 0157-0163.

(57) Rabi, I. I.: Space quantization in a gyrating magnetic field. Physical Review 1937, 51, 0652-0654.

(58) Rabi, I. I.; Zacharias, J. R.; Millman, S.; Kusch, P.: A new method of measuring nuclear magnetic moment. Physical Review 1938, 53, 318-318.

(59) Purcell, E. M.: Spontaneous Emission Probabilities at Radio Frequencies. Physical Review 1946, 69, 681-681.

(60) Torrey, H. C.; Purcell, E. M.; Pound, R. V.: Theory of Magnetic Resonance Absorption by Nuclear Moments in Solids. Physical Review 1946, 69, 680-680.

(61) Pound, R. V.; Purcell, E. M.: Measurement of Magnetic Resonance Absorption by Nuclear Moments in a Solid. Physical Review 1946, 69, 681-681.

(62) Purcell, E. M.; Torrey, H. C.; Pound, R. V.: Resonance Absorption by Nuclear Magnetic Moments in a Solid. Physical Review 1946, 69, 37-38.

(63) Bloch, F.; Hansen, W. W.; Packard, M.: The Nuclear Induction Experiment. Physical Review 1946, 70, 474-485.

(64) Bloch, F.; Hansen, W. W.; Packard, M.: Nuclear Induction. Physical Review 1946, 69, 127-127.

(65) Damadian, R.: Tumor Detection by Nuclear Magnetic Resonance. Science 1971, 171, 1151-\&.

(66) Lauterbur, P. C.: Image Formation by Induced Local Interactions Examples Employing Nuclear Magnetic-Resonance. Nature 1973, 242, 190-191.

(67) Damadian, R.: Nuclear Magnetic-Resonance - Noninvasive Approach to Cancer. Hosp Pract 1977, 12, 63-70.

(68) Damadian, R.; Goldsmith, M.; Minkoff, L.: Nmr in Cancer .16. Fonar Image of Live Human-Body. Physiological chemistry and physics 1977, 9, 97-\&.

(69) Damadian, R.; Minkoff, L.; Goldsmith, M.: Nmr in Cancer .21. Fonar Scan of the Live Human Abdomen. Physiological chemistry and physics 1978, 10, 561-563.

(70) Damadian, R.; Goldsmith, M.; Minkoff, L.: Nmr in Cancer .20. Fonar Scans of Patients with Cancer. Physiological chemistry and physics 1978, 10, 285-287.

(71) Celda, B.; Monleon, D.; Martinez-Bisbal, M. C.; Esteve, V.; MartinezGranados, B.; Pinero, E.; Ferrer, R.; Piquer, J.; Marti-Bonmati, L.; Cervera, J.: MRS as 
endogenous molecular imaging for brain and prostate tumors: FP6 project "eTUMOR". Advances in experimental medicine and biology 2006, 587, 285-302.

(72) Skinner, J. T.; Yankeelov, T. E.; Peterson, T. E.; Does, M. D.: Comparison of dynamic contrast-enhanced MRI and quantitative SPECT in a rat glioma model. Contrast Media Mol Imaging 2012, 7, 494-500.

(73) Yamada, T.; Maruoka, S.; Yamada, S.; Sonobe, M.: [Comparison of 201T1SPECT and MRI using Gd-DTPA for glioma]. Nihon Igaku Hoshasen Gakkai zasshi. Nippon acta radiologica 1999, 59, 402-8.

(74) Barber, P. A.; Consolo, H. K.; Yang, Q.; Darby, D. G.; Desmond, P. M.; Lichtenstein, M.; Tress, B. M.; Davis, S. M.: Comparison of MRI perfusion imaging and single photon emission computed tomography in chronic stroke. Cerebrovasc Dis 2001, $11,128-36$.

(75) Heckl, S.: Future contrast agents for molecular imaging in stroke. Current medicinal chemistry 2007, 14, 1713-28.

(76) Winter, C.; Bell, C.; Whyte, T.; Cardinal, J.; Macfarlane, D.; Rose, S.: Blood-brain barrier dysfunction following traumatic brain injury: correlation of $\mathrm{K}$ (trans) (DCE-MRI) and SUVR (99mTc-DTPA SPECT) but not serum S100B. Neurological research 2015, 37, 599-606.

(77) Maher, K.: Examples of T1 weighted, T2 weighted and PD weighted MRI scans., 2006.

(78) Dr Jeremy Jones and Prof Frank Gaillard, e. a.: MRI sequences (overview). Radiopaedia.org; Vol. 2017.

(79) Cohn, M.: Magnetic Resonance Studies of Metal-Enzyme-Substrate Interactions. Science 1962, 136, 325.

(80) Bloembergen, N.; Morgan, L. O.: Proton Relaxation Times in Paramagnetic Solutions. Effects of Electron Spin Relaxation. Journal of Chemical Physics 1961, 34, 842850 .

(81) Aime, S.; Botta, M.; Fasano, M.; Terreno, E.: Prototropic and WaterExchange Processes in Aqueous Solutions of Gd(III) Chelates. Accounts of Chemical Research 1999, 32, 941-949.

(82) Eisinger, J.; Shulman, R. G.; Blumberg, W. E.: Relaxation enhancement by paramagnetic ion binding in deoxyribonucleic acid solutions. Nature 1961, 192, 963-4.

(83) Bloch, F.: Nuclear Induction. Physical Review 1946, 70, 460-474.

(84) Caravan, P.; Ellison, J. J.; McMurry, T. J.; Lauffer, R. B.: Gadolinium(III) Chelates as MRI Contrast Agents: Structure, Dynamics, and Applications. Chemical Reviews 1999, 99, 2293-2352.

(85) Ward, K. M.; Aletras, A. H.; Balaban, R. S.: A New Class of Contrast Agents for MRI Based on Proton Chemical Exchange Dependent Saturation Transfer (CEST). Journal of Magnetic Resonance 2000, 143, 79-87.

(86) Hingorani, D. V.; Montano, L. A.; Randtke, E. A.; Lee, Y. S.; CardenasRodriguez, J.; Pagel, M. D.: A single diamagnetic catalyCEST MRI contrast agent that detects cathepsin B enzyme activity by using a ratio of two CEST signals. Contrast Media Mol Imaging 2016, 11, 130-8. 
(87) Longo, D. L.; Sun, P. Z.; Consolino, L.; Michelotti, F. C.; Uggeri, F.; Aime, S.: A general MRI-CEST ratiometric approach for $\mathrm{pH}$ imaging: demonstration of in vivo pH mapping with iobitridol. J Am Chem Soc 2014, 136, 14333-6.

(88) Delli Castelli, D.; Terreno, E.; Aime, S.: YbIII-HPDO3A: A Dual pH- and Temperature-Responsive CEST Agent. Angewandte Chemie International Edition 2011, 50, 1798-1800.

(89) Longo, D. L.; Busato, A.; Lanzardo, S.; Antico, F.; Aime, S.: Imaging the $\mathrm{pH}$ evolution of an acute kidney injury model by means of iopamidol, a MRI-CEST pHresponsive contrast agent. Magnetic Resonance in Medicine 2013, 70, 859-864.

(90) Zhang, S.; Malloy, C. R.; Sherry, A. D.: MRI Thermometry Based on PARACEST Agents. Journal of the American Chemical Society 2005, 127, 17572-17573.

(91) Aime, S.; Delli Castelli, D.; Fedeli, F.; Terreno, E.: A Paramagnetic MRICEST Agent Responsive to Lactate Concentration. Journal of the American Chemical Society 2002, 124, 9364-9365.

(92) Trokowski, R.; Ren, J.; Kálmán, F. K.; Sherry, A. D.: Selective Sensing of Zinc Ions with a PARACEST Contrast Agent. Angewandte Chemie International Edition 2005, 44, 6920-6923.

(93) Zhang, S.; Trokowski, R.; Sherry, A. D.: A Paramagnetic CEST Agent for Imaging Glucose by MRI. Journal of the American Chemical Society 2003, 125, 1528815289.

(94) Secondary Protein Structure. Structure, S. P., Ed., 2013.

(95) Dean Sherry, A.: Shifting Abilities of Ln3+ Ions. 2007.

(96) Dean Sherry, A.: Diamagnetic and Paramagnetic CEST. 2007.

(97) Aime, S.; Barge, A.; Botta, M.; De Sousa, A. S.; Parker, D.: Direct NMR Spectroscopic Observation of a Lanthanide-Coordinated Water Molecule whose Exchange Rate Is Dependent on the Conformation of the Complexes. Angewandte Chemie International Edition 1998, 37, 2673-2675.

(98) Zhang, S.; Winter, P.; Wu, K.; Sherry, A. D.: A Novel Europium(III)-Based MRI Contrast Agent. Journal of the American Chemical Society 2001, 123, 1517-1518.

(99) Sherry, A. D.; Woods, M.: Chemical Exchange Saturation Transfer Contrast Agents for Magnetic Resonance Imaging. Annual review of biomedical engineering 2008, 10, 391-411.

(100) Sherry, A. D.; Wu, Y.: The importance of water exchange rates in the design of responsive agents for MRI. Current Opinion in Chemical Biology 2013.

(101) Dean Sherry, A.: CEST Water Exchange Rate Changing With Pulse Power. 2007.

(102) Aime, S.; Barge, A.; Bruce, J. I.; Botta, M.; Howard, J. A. K.; Moloney, J. M.; Parker, D.; de Sousa, A. S.; Woods, M.: NMR, Relaxometric, and Structural Studies of the Hydration and Exchange Dynamics of Cationic Lanthanide Complexes of Macrocyclic Tetraamide Ligands. Journal of the American Chemical Society 1999, 121, 5762-5771.

(103) Ferrauto, G.; Delli Castelli, D.; Di Gregorio, E.; Terreno, E.; Aime, S.: LipoCEST and cellCEST imaging agents: opportunities and challenges. Wiley interdisciplinary reviews. Nanomedicine and nanobiotechnology 2016, 8, 602-18. 
(104) Terreno, E.; Boffa, C.; Menchise, V.; Fedeli, F.; Carrera, C.; Castelli, D. D.; Digilio, G.; Aime, S.: Gadolinium-doped LipoCEST agents: a potential novel class of dual 1H-MRI probes. Chem Commun (Camb) 2011, 47, 4667-9.

(105) Terreno, E.; Delli Castelli, D.; Violante, E.; Sanders, H. M.; Sommerdijk, N. A.; Aime, S.: Osmotically shrunken LIPOCEST agents: an innovative class of magnetic resonance imaging contrast media based on chemical exchange saturation transfer. Chemistry 2009, 15, 1440-8.

(106) Terreno, E.; Castelli, D. D.; Milone, L.; Rollet, S.; Stancanello, J.; Violante, E.; Aime, S.: First ex-vivo MRI co-localization of two LIPOCEST agents. Contrast Media Mol Imaging 2008, 3, 38-43.

(107) Shiho Sairenji, S. A., Tatsuya Nabeshima: Lanthanide Contraction. 2016.

(108) Electronic Configuration of Gd3+. 2017.

(109) Stephens, E. M.; Grisham, C. M.: Lithium-7 nuclear magnetic resonance, water proton nuclear magnetic resonance and gadolinium electron paramagnetic resonance studies of the sarcoplasmic reticulum calcium ion transport adenosine triphosphatase. Biochemistry 1979, 18, 4876-4885.

(110) Lauffer, R. B.: Paramagnetic Metal-Complexes as Water Proton Relaxation Agents for Nmr Imaging - Theory and Design. Chemical Reviews 1987, 87, 901-927.

(111) Bousquet, J. C.; Saini, S.; Stark, D. D.; Hahn, P. F.; Nigam, M.; Wittenberg, J.; Ferrucci, J. T., Jr.: Gd-DOTA: characterization of a new paramagnetic complex. Radiology 1988, 166, 693-8.

(112) Richard W. Taylor, R. A. B., Victor W Day, Kristin Bowman-James: Chelation Effect. 2012.

(113) Tweedle, M. F.; Eaton, S. M.; Eckelman, W. C.; Gaughan, G. T.; Hagan, J. J.; Wedeking, P. W.; Yost, F. J.: Comparative chemical structure and pharmacokinetics of MRI contrast agents. Investigative radiology 1988, 23 Suppl 1, S236-9.

(114) Meyer, M.; Dahaoui-Gindrey, V.; Lecomte, C.; Guilard, R.: Conformations and coordination schemes of carboxylate and carbamoyl derivatives of the tetraazamacrocycles cyclen and cyclam, and the relation to their protonation states. Coordination Chemistry Reviews 1998, 178-180, Part 2, 1313-1405.

(115) Dale, J.; Ryde-Petterson, G.; Strand, U.; Jalonen, H.; Lüning, B.; Swahn, C.-G.: Exploratory Calculations of Medium and Large Rings. Part 1. Conformational Minima of Cycloalkanes. Acta Chemica Scandinavica 1973, 27, 1115-1129.

(116) Marie-Rose Spirlet, J. R., Jean F. Desreux, Marie-France Loncin: Ideal 3,3,3,3 Ring Conformation. 1984.

(117) Spirlet, M. R.; Rebizant, J.; Desreux, J. F.; Loncin, M. F.: Crystal and molecular structure of sodium aqua(1,4,7,10-tetraazacyclododecane-1,4,7,10tetraacetato)europate(III) tetrahydrate $\mathrm{Na}+(\mathrm{EuDOTA} . \mathrm{H} 2 \mathrm{O})-.4 \mathrm{H} 2 \mathrm{O}$, and its relevance to NMR studies of the conformational behavior of the lanthanide complexes formed by the macrocyclic ligand DOTA. Inorganic Chemistry 1984, 23, 359-363.

(118) Desreux, J. F.: Nuclear magnetic resonance spectroscopy of lanthanide complexes with a tetraacetic tetraaza macrocycle. Unusual conformation properties. Inorganic Chemistry 1980, 19, 1319-1324. 
(119) Aime, S.; Botta, M.; Fasano, M.; Marques, M. P. M.; Geraldes, C. F. G. C.; Pubanz, D.; Merbach, A. E.: Conformational and Coordination Equilibria on DOTA Complexes of Lanthanide Metal Ions in Aqueous Solution Studied by 1H-NMR Spectroscopy. Inorganic Chemistry 1997, 36, 2059-2068.

(120) Brittain, H. G.; Desreux, J. F.: Luminescence and NMR studies of the conformational isomers of lanthanide complexes with an optically active polyaza polycarboxylic macrocycle. Inorganic Chemistry 1984, 23, 4459-4466.

(121) Howard, J. A. K.; Kenwright, A. M.; Moloney, J. M.; Parker, D.; Woods, M.; Port, M.; Navet, M.; Rousseau, O.: Structure and dynamics of all of the stereoisomers of europium complexes of tetra(carboxyethyl) derivatives of dota: ring inversion is decoupled from cooperative arm rotation in the RRRR and RRRS isomers. Chem. Commun. (Cambridge) 1998, 1381-1382.

(122) Aime, S.; Botta, M.; Ermondi, G.: NMR study of solution structures and dynamics of lanthanide(III) complexes of DOTA. Inorganic Chemistry 1992, 31, 42914299.

(123) Woods, M.; Kovacs, Z.; Kiraly, R.; Brücher, E.; Zhang, S.; Sherry, A. D.: Solution Dynamics and Stability of Lanthanide(III) (S)-2-(p-Nitrobenzyl)DOTA Complexes. Inorganic Chemistry 2004, 43, 2845-2851.

(124) Woods, M.; Aime, S.; Botta, M.; Howard, J. A. K.; Moloney, J. M.; Navet, M.; Parker, D.; Port, M.; Rousseaux, O.: Correlation of Water Exchange Rate with Isomeric Composition in Diastereoisomeric Gadolinium Complexes of Tetra(carboxyethyl)dota and Related Macrocyclic Ligands. Journal of the American Chemical Society 2000, 122, 9781-9792.

(125) Woods, M.; Kovacs, Z.; Zhang, S.; Sherry, A. D.: Towards the Rational Design of Magnetic Resonance Imaging Contrast Agents: Isolation of the Two Coordination Isomers of Lanthanide DOTA-Type Complexes. Angewandte Chemie International Edition 2003, 42, 5889-5892.

(126) Tircso, G.; Webber, B. C.; Kucera, B. E.; Young, V. G.; Woods, M.: Analysis of the Conformational Behavior and Stability of the SAP and TSAP Isomers of Lanthanide(III) NB-DOTA-Type Chelates. Inorganic Chemistry 2011, 50, 7966-7979.

(127) Webber, B. C.; Woods, M.: Structural Analysis of Isomeric Europium(III) Chelates of NB-DOTMA. Inorganic Chemistry 2012, 51, 8576-8582.

(128) Woods, M.; Botta, M.; Avedano, S.; Wang, J.; Sherry, A. D.: Towards the rational design of MRI contrast agents: a practical approach to the synthesis of gadolinium complexes that exhibit optimal water exchange. Dalton Transactions 2005, 0, 3829-3837.

(129) Carney, C. E.; Tran, A. D.; Wang, J.; Schabel, M. C.; Sherry, A. D.; Woods, M.: Towards the Rational Design of MRI Contrast Agents: $\delta$-Substitution of Lanthanide(III) NB-DOTA-Tetraamide Chelates Influences but Does Not Control Coordination Geometry. Chemistry - A European Journal 2011, 17, 10372-10378.

(130) Johns, C. S.; Swift, A. J.; Hughes, P. J.; Ohno, Y.; Schiebler, M.; Wild, J. M.: Pulmonary MR angiography and perfusion imaging-A review of methods and applications. European journal of radiology 2017, 86, 361-370. 
(131) Gaigalaite, V.; Vilimas, A.; Ozeraitiene, V.; Dementaviciene, J.; Janilionis, R.; Kalibatiene, D.; Rocka, S.: Association between vertebral artery hypoplasia and posterior circulation stroke. BMC neurology 2016, 16, 118.

(132) Kalvakuri, K.; Banga, S.; Upalakalin, N.; Shaw, C.; Davila, W. F.; Mungee, S.: Metastatic right ventricular mass with intracavitary obliteration. Journal of community hospital internal medicine perspectives 2016, 6, 31679.

(133) Fushimi, Y.; Okada, T.; Okuchi, S.; Yamamoto, A.; Kanagaki, M.; Fujimoto, K.; Togashi, K.: Jugular venous reflux on magnetic resonance angiography and radionuclide venography. Acta radiologica open 2016, 5, 2058460116681209.

(134) Dos Santos, R. G.; Osorio, F. L.; Crippa, J. A.; Hallak, J. E.: Classical hallucinogens and neuroimaging: A systematic review of human studies: Hallucinogens and neuroimaging. Neuroscience and biobehavioral reviews 2016, 71, 715-728.

(135) Jann, K.; Hernandez, L. M.; Beck-Pancer, D.; McCarron, R.; Smith, R. X.; Dapretto, M.; Wang, D. J.: Altered resting perfusion and functional connectivity of default mode network in youth with autism spectrum disorder. Brain and behavior 2015, 5, $\mathrm{e} 00358$.

(136) Cai, K.; Tain, R. W.; Zhou, X. J.; Damen, F. C.; Scotti, A. M.; Hariharan, H.; Poptani, H.; Reddy, R.: Creatine CEST MRI for Differentiating Gliomas with Different Degrees of Aggressiveness. Molecular imaging and biology : MIB : the official publication of the Academy of Molecular Imaging 2017, 19, 225-232.

(137) Plotkin, M.; Eisenacher, J.; Bruhn, H.; Wurm, R.; Michel, R.; Stockhammer, F.; Feussner, A.; Dudeck, O.; Wust, P.; Felix, R.; Amthauer, H.: 123I-IMT SPECT and $1 \mathrm{H}$ MR-spectroscopy at $3.0 \mathrm{~T}$ in the differential diagnosis of recurrent or residual gliomas: a comparative study. Journal of neuro-oncology 2004, 70, 49-58.

(138) Yang, M.; Sun, J.; Bai, H. X.; Tao, Y.; Tang, X.; States, L. J.; Zhang, Z.; Zhou, J.; Farwell, M. D.; Zhang, P.; Xiao, B.; Yang, L.: Diagnostic accuracy of SPECT, PET, and MRS for primary central nervous system lymphoma in HIV patients: A systematic review and meta-analysis. Medicine 2017, 96, e6676.

(139) Peterson, P. L.; Axford, J. S.; Isenberg, D.: Imaging in CNS lupus. Best practice \& research. Clinical rheumatology 2005, 19, 727-39.

(140) Moretti, A.; Gorini, A.; Villa, R. F.: Affective disorders, antidepressant drugs and brain metabolism. Molecular psychiatry 2003, 8, 773-85.

(141) Flugge, T.; Hovener, J. B.; Ludwig, U.; Eisenbeiss, A. K.; Spittau, B.; Hennig, J.; Schmelzeisen, R.; Nelson, K.: Magnetic resonance imaging of intraoral hard and soft tissues using an intraoral coil and FLASH sequences. European radiology 2016, $26,4616-4623$.

Zollner, D. F.: MRA Image. 2017.

fMRI Image.

(145) Daryaei, I.; Jones, K. M.; Pagel, M. D.: Detection of DT-diaphorase Enzyme with a ParaCEST MRI Contrast Agent. Chemistry 2017, 23, 6514-6517.

(146) Sinharay, S.; Randtke, E. A.; Jones, K. M.; Howison, C. M.; Chambers, S. K.; Kobayashi, H.; Pagel, M. D.: Noninvasive detection of enzyme activity in tumor 
models of human ovarian cancer using catalyCEST MRI. Magn Reson Med 2017, 77, 2005-2014.

(147) Daryaei, I.; Randtke, E. A.; Pagel, M. D.: A biomarker-responsive T2ex MRI contrast agent. Magn Reson Med 2017, 77, 1665-1670.

(148) Longo, D. L.; Bartoli, A.; Consolino, L.; Bardini, P.; Arena, F.; Schwaiger, M.; Aime, S.: In Vivo Imaging of Tumor Metabolism and Acidosis by Combining PET and MRI-CEST pH Imaging. Cancer research 2016, 76, 6463-6470.

(149) Liu, G.; Liang, Y.; Bar-Shir, A.; Chan, K. W.; Galpoththawela, C. S.; Bernard, S. M.; Tse, T.; Yadav, N. N.; Walczak, P.; McMahon, M. T.; Bulte, J. W.; van Zijl, P. C.; Gilad, A. A.: Monitoring enzyme activity using a diamagnetic chemical exchange saturation transfer magnetic resonance imaging contrast agent. J Am Chem Soc 2011, 133, 16326-9.

(150) De Leon-Rodriguez, L. M.; Lubag, A. J.; Malloy, C. R.; Martinez, G. V.; Gillies, R. J.; Sherry, A. D.: Responsive MRI agents for sensing metabolism in vivo. Acc Chem Res 2009, 42, 948-57.

(151) Tan, M.; Lu, Z. R.: Integrin Targeted MR Imaging. Theranostics 2011, 1, 83-101.

(152) Fernandez-Cuervo, G.; Tucker, K. A.; Malm, S. W.; Jones, K. M.; Pagel, M. D.: Diamagnetic Imaging Agents with a Modular Chemical Design for Quantitative Detection of beta-Galactosidase and beta-Glucuronidase Activities with CatalyCEST MRI. Bioconjug Chem 2016.

(153) Ngen, E. J.; Bar-Shir, A.; Jablonska, A.; Liu, G.; Song, X.; Ansari, R.; Bulte, J. W.; Janowski, M.; Pearl, M.; Walczak, P.; Gilad, A. A.: Imaging the DNA Alkylator Melphalan by CEST MRI: An Advanced Approach to Theranostics. Molecular pharmaceutics 2016, 13, 3043-53.

(154) Shazeeb, M. S.; Gupta, S.; Bogdanov, A., Jr.: MR signal amplification for imaging of the mutant EGF receptor in orthotopic human glioma model. Molecular imaging and biology : MIB : the official publication of the Academy of Molecular Imaging 2013, 15, 675-84.

(155) Flament, J.; Geffroy, F.; Medina, C.; Robic, C.; Mayer, J. F.; Meriaux, S.; Valette, J.; Robert, P.; Port, M.; Le Bihan, D.; Lethimonnier, F.; Boumezbeur, F.: In vivo CEST MR imaging of U87 mice brain tumor angiogenesis using targeted LipoCEST contrast agent at 7 T. Magn Reson Med 2013, 69, 179-87.

(156) Lindenberg, L.; Ahlman, M.; Turkbey, B.; Mena, E.; Choyke, P.: Advancement of MR and PET/MR in Prostate Cancer. Seminars in nuclear medicine 2016, $46,536-543$.

(157) Liu, H.; Jablonska, A.; Li, Y.; Cao, S.; Liu, D.; Chen, H.; Van Zijl, P. C.; Bulte, J. W.; Janowski, M.; Walczak, P.; Liu, G.: Label-free CEST MRI Detection of Citicoline-Liposome Drug Delivery in Ischemic Stroke. Theranostics 2016, 6, 1588-600.

(158) Benz, M. R.; Vargas, H. A.; Sala, E.: Functional MR Imaging Techniques in Oncology in the Era of Personalized Medicine. Magnetic resonance imaging clinics of North America 2016, 24, 1-10. 
(159) Jin, T.; Wang, P.; Hitchens, T. K.; Kim, S. G.: Enhancing sensitivity of pHweighted MRI with combination of amide and guanidyl CEST. NeuroImage 2017, 157, 341-350.

(160) Chen, M.; Chen, C.; Shen, Z.; Zhang, X.; Chen, Y.; Lin, F.; Ma, X.; Zhuang, C.; Mao, Y.; Gan, H.; Chen, P.; Zong, X.; Wu, R.: Extracellular pH is a biomarker enabling detection of breast cancer and liver cancer using CEST MRI. Oncotarget 2017.

(161) Longo, D. L.; Cutrin, J. C.; Michelotti, F.; Irrera, P.; Aime, S.: Noninvasive evaluation of renal $\mathrm{pH}$ homeostasis after ischemia reperfusion injury by CEST-MRI. NMR Biomed 2017, 30 .

(162) Tsitovich, P. B.; Cox, J. M.; Spernyak, J. A.; Morrow, J. R.: Gear Up for a pH Shift: A Responsive Iron(II) 2-Amino-6-picolyl-Appended Macrocyclic paraCEST Agent That Protonates at a Pendent Group. Inorg Chem 2016, 55, 12001-12010.

(163) Bagga, P.; Crescenzi, R.; Krishnamoorthy, G.; Verma, G.; Nanga, R. P.; Reddy, D.; Greenberg, J.; Detre, J. A.; Hariharan, H.; Reddy, R.: Mapping the alterations in glutamate with GluCEST MRI in a mouse model of dopamine deficiency. Journal of neurochemistry 2016, 139, 432-439.

(164) Jones, K. M.; Randtke, E. A.; Howison, C. M.; Pagel, M. D.: Respiration gating and Bloch fitting improve $\mathrm{pH}$ measurements with acidoCEST MRI in an ovarian orthotopic tumor model. Proceedings of SPIE--the International Society for Optical Engineering 2016, 9788.

(165) Yang, X.; Song, X.; Ray Banerjee, S.; Li, Y.; Byun, Y.; Liu, G.; Bhujwalla, Z. M.; Pomper, M. G.; McMahon, M. T.: Developing imidazoles as CEST MRI pH sensors. Contrast Media Mol Imaging 2016, 11, 304-12.

(166) Zu, Z.; Louie, E. A.; Lin, E. C.; Jiang, X.; Does, M. D.; Gore, J. C.; Gochberg, D. F.: Chemical exchange rotation transfer imaging of intermediate-exchanging amines at 2 ppm. NMR Biomed 2017.

(167) Zu, Z.; Li, H.; Jiang, X.; Gore, J. C.: Spin-lock imaging of exogenous exchange-based contrast agents to assess tissue $\mathrm{pH}$. Magn Reson Med 2017.

(168) Khlebnikov, V.; Windschuh, J.; Siero, J. C.; Zaiss, M.; Luijten, P. R.; Klomp, D. W.; Hoogduin, H.: On the transmit field inhomogeneity correction of relaxationcompensated amide and NOE CEST effects at 7 T. NMR Biomed 2017, 30.

(169) Randtke, E. A.; Granados, J. C.; Howison, C. M.; Pagel, M. D.; CardenasRodriguez, J.: Multislice CEST MRI improves the spatial assessment of tumor $\mathrm{pH}$. Magn Reson Med 2017, 78, 97-106.

(170) Khlebnikov, V.; Geades, N.; Klomp, D. W. J.; Hoogduin, H.; Gowland, P.; Mougin, O.: Comparison of pulsed three-dimensional CEST acquisition schemes at 7 tesla: steady state versus pseudosteady state. Magn Reson Med 2017, 77, 2280-2287.

(171) Zhang, Y.; Heo, H. Y.; Lee, D. H.; Bottomley, P.; Zhou, J.: Fast Chemical Exchange Saturation Transfer (CEST) Imaging with Variably-accelerated Sensitivity Encoding (vSENSE). Proceedings of the International Society for Magnetic Resonance in Medicine ... Scientific Meeting and Exhibition. International Society for Magnetic Resonance in Medicine. 2016, 24, 1522.

(172) Jones, K. M.; Randtke, E. A.; Yoshimaru, E. S.; Howison, C. M.; Chalasani, P.; Klein, R. R.; Chambers, S. K.; Kuo, P. H.; Pagel, M. D.: Clinical Translation of Tumor 
Acidosis Measurements with AcidoCEST MRI. Molecular imaging and biology : MIB : the official publication of the Academy of Molecular Imaging 2016.

(173) Zhou, I. Y.; Fuss, T. L.; Igarashi, T.; Jiang, W.; Zhou, X.; Cheng, L. L.; Sun, P. Z.: Tissue Characterization with Quantitative High-Resolution Magic Angle Spinning Chemical Exchange Saturation Transfer Z-Spectroscopy. Anal Chem 2016, 88, 10379-10383.

(174) Jiang, W.; Zhou, I. Y.; Wen, L.; Zhou, X.; Sun, P. Z.: A theoretical analysis of chemical exchange saturation transfer echo planar imaging (CEST-EPI) steady state solution and the CEST sensitivity efficiency-based optimization approach. Contrast Media Mol Imaging 2016, 11, 415-423.

(175) Tain, R.; Xu, H. N.; Zhou, X. J.; Li, L. Z.; Cai, K.: Magnetization Transfer MRI Contrast May Correlate with Tissue Redox State in Prostate Cancer. Advances in experimental medicine and biology 2016, 923, 401-6.

(176) Randtke, E. A.; Pagel, M. D.; Cardenas-Rodriguez, J.: QUESPOWR MRI: QUantification of Exchange as a function of Saturation Power On the Water Resonance. $J$ Magn Reson 2016, 270, 56-70.

(177) Schmidt, H.; Schwenzer, N. F.; Gatidis, S.; Kustner, T.; Nikolaou, K.; Schick, F.; Martirosian, P.: Systematic Evaluation of Amide Proton Chemical Exchange Saturation Transfer at $3 \mathrm{~T}$ : Effects of Protein Concentration, $\mathrm{pH}$, and Acquisition Parameters. Investigative radiology 2016, 51, 635-46.

(178) Aime, S.; Delli Castelli, D.; Terreno, E.: Highly Sensitive MRI Chemical Exchange Saturation Transfer Agents Using Liposomes. Angewandte Chemie International Edition 2005, 44, 5513-5515.

(179) Biology, O.: Lipid Structure. Khan Academy.

(180) Tagami, T.; Yanai, H.; Terada, Y.; Ozeki, T.: Evaluation of Phosphatidylserine-Specific Peptide-Conjugated Liposomes Using a Model System of Malaria-Infected Erythrocytes. Biological \& pharmaceutical bulletin 2015, 38, 1649-51.

(181) Kirsch, T.; Harrison, G.; Golub, E. E.; Nah, H. D.: The roles of annexins and types II and X collagen in matrix vesicle-mediated mineralization of growth plate cartilage. The Journal of biological chemistry 2000, 275, 35577-83.

(182) Kim, J. G.; Kim, J. D.: Vesicle to micelle transitions of egg phosphatidylcholine liposomes induced by nonionic surfactants, poly(oxyethylene) cetyl ethers. Journal of biochemistry 1991, 110, 436-42.

(183) Kamps, J. A.; Morselt, H. W.; Scherphof, G. L.: Uptake of liposomes containing phosphatidylserine by liver cells in vivo and by sinusoidal liver cells in primary culture: in vivo-in vitro differences. Biochemical and biophysical research communications 1999, 256, 57-62.

(184) Bhagat, M.; Sofou, S.: Membrane heterogeneities and fusogenicity in phosphatidylcholine-phosphatidic acid rigid vesicles as a function of $\mathrm{pH}$ and lipid chain mismatch. Langmuir 2010, 26, 1666-73.

(185) Tabaei, S. R.; Jackman, J. A.; Kim, S. O.; Zhdanov, V. P.; Cho, N. J.: Solvent-assisted lipid self-assembly at hydrophilic surfaces: factors influencing the formation of supported membranes. Langmuir 2015, 31, 3125-34. 
(186) van Rooijen, B. D.; Claessens, M. M.; Subramaniam, V.: Lipid bilayer disruption by oligomeric alpha-synuclein depends on bilayer charge and accessibility of the hydrophobic core. Biochimica et biophysica acta 2009, 1788, 1271-8.

(187) Chrzeszczyk, A.; Wishnia, A.; Springer, C. S., Jr.: The intrinsic structural asymmetry of highly curved phospholipid bilayer membranes. Biochimica et biophysica acta 1977, 470, 161-9.

(188) Gløgård, C.; Stensrud, G.; Hovland, R.; Fossheim, S. L.; Klaveness, J.: Liposomes as carriers of amphiphilic gadolinium chelates: the effect of membrane composition on incorporation efficacy and in vitro relaxivity. International Journal of Pharmaceutics 2002, 233, 131-140.

(189) Papahadjopoulos, D.; Moscarello, M.; Eylar, E. H.; Isac, T.: Effects of proteins on thermotropic phase transitions of phospholipid membranes. Biochimica et biophysica acta 1975, 401, 317-35.

(190) Cullis, P. R.: Lateral diffusion rates of phosphatidylcholine in vesicle membranes: effects of cholesterol and hydrocarbon phase transitions. FEBS letters 1976, $70,223-8$.

(191) Lawaczeck, R.; Kainosho, M.; Chan, S. I.: The formation and annealing of structural defects in lipid bilayer vesicles. Biochimica et biophysica acta 1976, 443, 31330 .

(192) Spiker, R. C.; Levin, I. W.: Phase transitions of phospholipid single-wall vesicles and multilayers. Measurement by vibrational Raman spectroscopic frequency differences. Biochimica et biophysica acta 1976, 433, 457-68.

(193) Martin, F. J.; MacDonald, R. C.: Phospholipid exchange between bilayer membrane vesicles. Biochemistry 1976, 15, 321-7.

(194) Duckwitz-peterlein, G.; Eilenberger, G.; Overath, P.: Phospholipid exchange between bilayer membranes. Biochimica et biophysica acta 1977, 469, 311-25.

(195) Avramovic-Zikic, O.; Colbow, K.: Turbidity changes of lipid vesicles near the phase transition temperature as an indication of fusion. Biochimica et biophysica acta 1978, 512, 97-104.

(196) Scherphof, G.; Morselt, H.; Regts, J.; Wilschut, J. C.: The involvement of the lipid phase transition in the plasma-induced dissolution of multilamellar phosphatidylcholine vesicles. Biochimica et biophysica acta 1979, 556, 196-207.

(197) Koga, K.; Kanazawa, Y.: Dynamical structure of phosphatidylcholine molecules in single bilayer vesicles observed by nitrogen-14 nuclear magnetic resonance. Biochemistry 1980, 19, 2779-83.

(198) Lewis, B. A.; Engelman, D. M.: Lipid bilayer thickness varies linearly with acyl chain length in fluid phosphatidylcholine vesicles. Journal of molecular biology 1983, $166,211-7$.

(199) Massari, S.; Colonna, R.: Lipid miscibility and size increase of vesicles composed of two phosphatidylcholines. Biochimica et biophysica acta 1986, 863, 264-76.

(200) Beckmann, M.; Nollert, P.; Kolb, H. A.: Manipulation and molecular resolution of a phosphatidylcholine-supported planar bilayer by atomic force microscopy. The Journal of membrane biology 1998, 161, 227-33. 
(201) Campbell, R. B.; Balasubramanian, S. V.; Straubinger, R. M.: Phospholipid-cationic lipid interactions: influences on membrane and vesicle properties. Biochimica et biophysica acta 2001, 1512, 27-39.

(202) Bui, H. H.; Khaledi, M. G.: Determination of vesicle-water partition coefficients by electrokinetic chromatography: study of temperature effect. Journal of colloid and interface science 2002, 253, 397-401.

(203) Yang, L.; Kindt, J. T.: Simulation Study of the Permeability of a Model Lipid Membrane at the Fluid-Solid Phase Transition. Langmuir 2015, 31, 2187-2195.

(204) Jing Li, X. W., Ting Zhang, Chunling Wang, Zhenjun Huang, Xiang Luo, Yihui Deng: Phase Transition Temperatures. 2015.

(205) Pal, R.; Barenholz, Y.; Wagner, R. R.: Effect of cholesterol concentration on organization of viral and vesicle membranes. Probed by accessibility to cholesterol oxidase. The Journal of biological chemistry 1980, 255, 5802-6.

(206) Chahid, B.; Vander Elst, L.; Flament, J.; Boumezbeur, F.; Medina, C.; Port, M.; Muller, R. N.; Lesieur, S.: Entrapment of a neutral Tm(III)-based complex with two inner-sphere coordinated water molecules into PEG-stabilized vesicles: towards an alternative strategy to develop high-performance LipoCEST contrast agents for MR imaging. Contrast Media \& Molecular Imaging 2014, 9, 391-399.

(207) Gent, M. P.; Prestegard, J. H.: Cholesterol-phosphatidylcholine interactions in vesicle systems. Implication of vesicle size and proton magnetic resonance line-width changes. Biochemistry 1974, 13, 4027-33.

(208) Newman, G. C.; Huang, C.: Structural studies on phophatidylcholinecholesterol mixed vesicles. Biochemistry 1975, 14, 3363-70.

(209) Haran, N.; Shoporer, M.: Study of water permeability through phospholipid vesicle membranes by $17 \mathrm{O}$ NMR. Biochimica et biophysica acta 1976, 426, 638-46.

2016.

(210) Himanshu Pandey, R. R., Vishnu Agarwal: Vesicle Classification Image.

(211) Avanti Polar Lipids, I.: Liposome Preparation. 2017.

(212) Patil, Y. P.; Jadhav, S.: Novel methods for liposome preparation. Chemistry and physics of lipids 2014, 177, 8-18.

(213) Malinovskaja-Gomez, K.; Espuelas, S.; Garrido, M. J.; Hirvonen, J.; Laaksonen, T.: Comparison of liposomal drug formulations for transdermal iontophoretic drug delivery. European journal of pharmaceutical sciences : official journal of the European Federation for Pharmaceutical Sciences 2017, 106, 294-301.

(214) Matos, A. L. L.; Pereira, G.; Cabral Filho, P. E.; Santos, B. S.; Fontes, A.: Delivery of cationic quantum dots using fusogenic liposomes in living cells. Journal of photochemistry and photobiology. B, Biology 2017, 171, 43-49.

(215) Lee, J.; Lee, H.; Goh, U.; Kim, J.; Jeong, M.; Park, J. H.: Cellular Engineering with Membrane Fusogenic Liposomes to Produce Functionalized Extracellular Vesicles. ACS applied materials \& interfaces 2016, 8, 6790-5.

(216) Valizadeh, H.; Ghanbarzadeh, S.; Zakeri-Milani, P.: Fusogenic liposomal formulation of sirolimus: improvement of drug anti-proliferative effect on human T-cells. Drug development and industrial pharmacy 2015, 41, 1558-65. 
(217) Ghanbarzadeh, S.; Khorrami, A.; Mohamed Khosroshahi, L.; Arami, S.: Fusogenic $\mathrm{pH}$ sensitive liposomal formulation for rapamycin: improvement of antiproliferative effect. Pharmaceutical biology 2014, 52, 848-54.

(218) Bulbake, U.; Doppalapudi, S.; Kommineni, N.; Khan, W.: Liposomal Formulations in Clinical Use: An Updated Review. Pharmaceutics 2017, 9.

(219) Boyd, B. J.; Galle, A.; Daglas, M.; Rosenfeld, J. V.; Medcalf, R.: Traumatic brain injury opens blood-brain barrier to stealth liposomes via an enhanced permeability and retention (EPR)-like effect. Journal of drug targeting 2015, 23, 847-53.

(220) Naicker, K.; Ariatti, M.; Singh, M.: PEGylated galactosylated cationic liposomes for hepatocytic gene delivery. Colloids and surfaces. B, Biointerfaces 2014, 122, 482-90.

(221) Noble, G. T.; Stefanick, J. F.; Ashley, J. D.; Kiziltepe, T.; Bilgicer, B.: Ligand-targeted liposome design: challenges and fundamental considerations. Trends in biotechnology 2014, 32, 32-45.

(222) Pujol-Autonell, I.; Mansilla, M. J.; Rodriguez-Fernandez, S.; Cano-Sarabia, M.; Navarro-Barriuso, J.; Ampudia, R. M.; Rius, A.; Garcia-Jimeno, S.; Perna-Barrull, D.; Martinez-Caceres, E.; Maspoch, D.; Vives-Pi, M.: Liposome-based immunotherapy against autoimmune diseases: therapeutic effect on multiple sclerosis. Nanomedicine (Lond) 2017, 12, 1231-1242.

(223) Pang, L.; Macauley, M. S.; Arlian, B. M.; Nycholat, C. M.; Paulson, J. C.: Encapsulating an Immunosuppressant Enhances Tolerance Induction by Siglec-Engaging Tolerogenic Liposomes. Chembiochem : a European journal of chemical biology 2017, $18,1226-1233$.

(224) Serrats, J.; Grigoleit, J. S.; Alvarez-Salas, E.; Sawchenko, P. E.: Proinflammatory immune-to-brain signaling is involved in neuroendocrine responses to acute emotional stress. Brain, behavior, and immunity 2017, 62, 53-63.

(225) Perrie, Y.; Crofts, F.; Devitt, A.; Griffiths, H. R.; Kastner, E.; Nadella, V.: Designing liposomal adjuvants for the next generation of vaccines. Advanced drug delivery reviews 2016, 99, 85-96.

(226) Cao, Y.; Yi, J.; Yang, X.; Liu, L.; Yu, C.; Huang, Y.; Sun, L.; Bao, Y.; Li, Y.: Efficient cancer regression by a thermosensitive liposome for Photoacoustic ImagingGuided Photothermal/Chemo Combinatorial Therapy. Biomacromolecules 2017.

(227) Mazur, F.; Bally, M.; Stadler, B.; Chandrawati, R.: Liposomes and lipid bilayers in biosensors. Advances in colloid and interface science 2017.

(228) Alavizadeh, S. H.; Gheybi, F.; Nikpoor, A. R.; Badiee, A.; Golmohammadzadeh, S.; Jaafari, M. R.: Therapeutic Efficacy of Cisplatin Thermosensitive Liposomes upon Mild Hyperthermia in C26 Tumor Bearing BALB/c Mice. Molecular pharmaceutics 2017, 14, 712-721.

(229) Silva, J. O.; Fernandes, R. S.; Lopes, S. C.; Cardoso, V. N.; Leite, E. A.; Cassali, G. D.; Marzola, M. C.; Rubello, D.; Oliveira, M. C.; de Barros, A. L.: pHSensitive, Long-Circulating Liposomes as an Alternative Tool to Deliver Doxorubicin into Tumors: a Feasibility Animal Study. Molecular imaging and biology : MIB : the official publication of the Academy of Molecular Imaging 2016, 18, 898-904. 
(230) Giansanti, L.; Mauceri, A.; Galantini, L.; Altieri, B.; Piozzi, A.; Mancini, G.: Glucosylated $\mathrm{pH}$-sensitive liposomes as potential drug delivery systems. Chemistry and physics of lipids 2016, 200, 113-119.

(231) Pezzoli, D.; Tallarita, E.; Rosini, E.; Candiani, G.: Characterization and Investigation of Redox-Sensitive Liposomes for Gene Delivery. Methods Mol Biol 2016, 1445, 217-33.

(232) TEM Image of MLVs.

(233) Enzo Terreno, A. B., Lorena Beltrami, Giancarlo Cravotto, Daniela Delli Castelli, Franco Fedeli, Bhagavathsingh Jebasingh, Silvio Aime: LipoCEST Image. 2008.

(234) Carney, C. E.; Tran, A. D.; Wang, J.; Schabel, M. C.; Sherry, A. D.; Woods, M.: Towards the Rational Design of MRI Contrast Agents: $\delta$-Substitution of Lanthanide(III) NB-DOTA-Tetraamide Chelates Influences but Does Not Control Coordination Geometry. Chemistry - A European Journal 2011, 17, 10372-10378.

(235) Slack, J.; Woods, M.: The effect of regioisomerism on the coordination chemistry and CEST properties of lanthanide(III) NB-DOTA-tetraamide chelates. $J$ Biol Inorg Chem 2014, 19, 173-189.

(236) Zhao, J. M.; Har-el, Y.-e.; McMahon, M. T.; Zhou, J.; Sherry, A. D.; Sgouros, G.; Bulte, J. W. M.; van Zijl, P. C. M.: Size-Induced Enhancement of Chemical Exchange Saturation Transfer (CEST) Contrast in Liposomes. Journal of the American Chemical Society 2008, 130, 5178-5184.

(237) Tilcock, C.; Unger, E.; Cullis, P.; MacDougall, P.: Liposomal Gd-DTPA: preparation and characterization of relaxivity. Radiology 1989, 171, 77-80.

(238) Unger, E. C.; MacDougall, P.; Cullis, P.; Tilcock, C.: Liposomal GdDTPA: effect of encapsulation on enhancement of hepatoma model by MRI. Magnetic resonance imaging 1989, 7, 417-23.

(239) Donahue, K. M.; Burstein, D.; Manning, W. J.; Gray, M. L.: Studies of Gd-DTPA relaxivity and proton exchange rates in tissue. Magn Reson Med 1994, 32, 6676. 